Published as:

MacGowan, S. A.; Senge, M. O. (2011):

Conformational control of cofactors in nature-functional tetrapyrrole conformations in the photosynthetic reaction centers of purple bacteria. Chemical Communications 47, 11621-11623. 


\title{
Conformational control of cofactors in nature - Functional tetrapyrrole conformations in the photosynthetic reaction centers of purple bacteria
}

\author{
Stuart A. MacGowan and Mathias O. Senge ${ }^{*}$, \\ Received (in XXX, XXX) Xth XXXXXXXXX 20XX, Accepted Xth XXXXXXXXX 20XX \\ ${ }_{10}$ DOI: 10.1039/b000000x
}

\begin{abstract}
Chemically identical tetrapyrrole cofactors such as hemes and chlorophylls participate in functionally diverse biological roles. An analysis of the available protein structural data for the bacteriochlorophylls in the photosynthetic reaction center gives ${ }_{15}$ statistically reliable evidence of the hypothesis that the protein induced cofactor conformation is a modulator of the biomolecular function of each reaction center. The results serve as a general model to illustrate conformational control of tetrapyrrole cofactors in other proteins.
\end{abstract}

20 Tetrapyrrole containing proteins are one of the most fundamental classes of enzymes found in nature and it is an open question to give a chemical rationale for the multitude of biological reactions that can be catalyzed by these pigment-protein complexes. ${ }^{1} \mathrm{~A}$ critical factor for all biological functions is the close structural 25 interplay between bound cofactors and the respective apoprotein in addition to factors such as hydrogen bonding or electronic effects. In individual tetrapyrrolic systems there is considerable evidence ${ }^{2}$ that nonplanarity is the key geometric factor affecting their physicochemical properties. Thus, the biological diversity of 30 chemically identical tetrapyrroles has been linked with distinct,

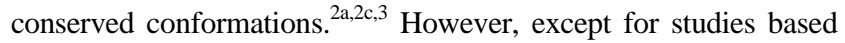
on individual structures, no convincing general proof has been given for this concept of conformational control thus far.

Using the cofactors of the photosynthetic reaction center in 35 purple bacteria we present the first statistically reliable analysis of such systems for this hypothesis and show that the various chromophores of the reaction center have different conformations which can be correlated with their chemical role. This shows that the protein scaffold exerts functional conformational control on the 40 porphyrin macrocycle and that modulation of the macrocycle conformation is an effective and general means to fine-tune the cofactor properties in vivo and to utilize the same cofactor for different chemical reactions. The results serve as a general model for the structural factors involved in cofactor regulation in nature.

45 The initial steps of photosynthesis take place in the reaction center (RC); the processes of light-harvesting, charge-separation and photoprotection all involve and indeed depend upon numerous tetrapyrrole cofactors. ${ }^{4}$ In fact, the RC is itself a true testament to the versatility of these cofactors where chemically 50 identical species perform one or more of these highly specialized functions within the same protein. The focus here is on the six tetrapyrrole cofactors of the electron transfer chain (ETC) from the RCs of the purple photosynthetic bacteria (Fig. 1). The cofactors of the ETC are anchored to one of two reaction center 55 proteins ( $\mathrm{L}$ or $\mathrm{M}$ ), via numerous interactions and are arranged in pairs of approximate $\mathrm{C}_{2}$ symmetry. ${ }^{[5]}$ An interesting enigma, addressed further in this article, is the preferential electron transfer along the L-branch. ${ }^{[6]}$
60 In the past conformational aspects of porphyrin structures have been described using typical core structural data, such as bond lengths, tilt angles, skeletal deviation plots or out-of-plane displacements which gave rise to the identification of some general types of macrocycle conformations (e.g. ruffled, doomed, ${ }_{65}$ saddle) ${ }^{2 a, 8}$ However, these data do not lend itself to a quantitative analysis of chemically very distinct species and so here we use the method of normal-coordinate structural decomposition (NSD) $^{3 \mathrm{~b}, 9}$ which overcomes this issue. This technique was developed by Shelnutt and affords clear separation of the 70 contributing distortions (normal-modes) to the macrocycle conformation in a quantitative fashion allowing a comparative analysis of conformational effects in porphyrins and is valid for use with the porphyrinoid cofactors in protein structures. ${ }^{3 \mathrm{~b}, 9 \mathrm{~b}}$

Our approach involved the determination of the NSD for 75 every ETC cofactor [bacteriochlorophyll (BChl) and bacteriopheophytin (BPhe)] of photosynthetic bacteria from relevant crystal structures obtained from the Protein Data Bank (PDB; structure overview Table 1). Via a methodical statistical route, including investigation of the nature and reliability of each 80 crystal structure, we refined the dataset and extracted the best estimate of the actual conformations present in the crystal.

Table 1. Summary of RC crystal structures (details in Section 6, S.I.)

\begin{tabular}{lll}
\hline Species & Crystal structures $^{\mathrm{a}}$ & Resolution(s) $^{\mathrm{b}}$ \\
\hline B. viridis & $15(14)$ & $1.86-3.21(2.36)$ \\
R. sphaeroides & $77(27)$ & $1.80-4.60(2.66)$ \\
\hline${ }^{\mathrm{a}}$ Independent RC crystal structures assessed (retained for best estimate in
\end{tabular}

parenthesis) ${ }^{\mathrm{b}}$ Resolution range (mean) of all structures.

85 Agglomerative hierarchical clustering of the cofactor conformations revealed that their major determinant was indeed their functional position in the ETC. However, closer inspection indicated secondary features that were related to entire crystal structures and we sought to assess the experimental correlations 90 that were present and so performed further analyses where the conformations of each cofactor reflected each structure's observable variables.

In the $R$. sphaeroides data, the inter-crystal structure Euclidean dissimilarities highlighted that a number of structures 95 did not exhibit any degree of experimental variation (Fig. 2) and were therefore removed them further consideration; it is likely that they originate from the practice of rigid-body refinement. Next, a two-factor analysis revealed that structures exhibited either: planar cofactors, functionally distinct nonplanar cofactors 100 or functionally distinct nonplanar cofactors with unusual in-plane contractions (Fig. 2). Based on a comparison to high-resolution chlorin analogues from the Cambridge Structural Database ${ }^{10}$ and others reasons we concluded that the first nonplanar set mentioned above presents the most reliable cofactor 105 conformations. The NSD results of these structures' cofactors (Fig. 3) show clearly the distinct conformations present at each position of the ETC. Notably, the absolute nature of this 
conformational superstructure varies with species but retains some general features and in some cases almost conserved 110 conformations.
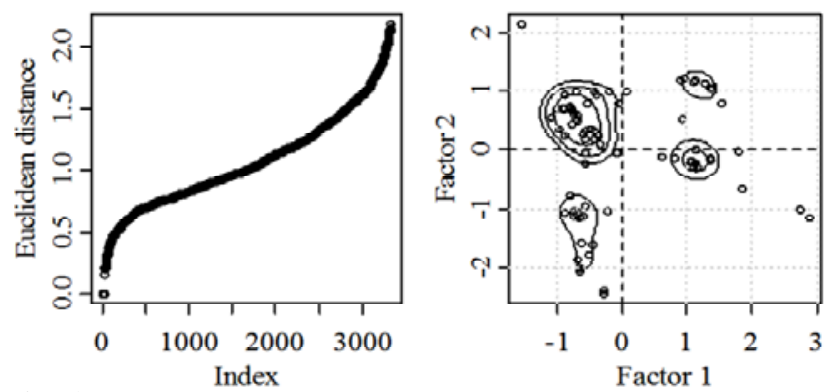

Fig. 2 Analyses of experimental effects in $R$. sphaeroides crystal structures. [Left] Euclidean dissimilarities between each structures’ BChl conformations; cluster near zero indicates structures that exhibit no 115 experimental variation. [Right] Plot of PDB structure scores on the Varimax rotated factors, computed by the regression method with kernel density contours overlaid. F1 (22.6\% total variance) correlates with increasing planarity and F2 (21.5\%) negatively with in-plane contraction.

In general, the deformation of the special pair cofactors is 120 always the greatest (of at least the bacteriochlorophylls (BChls)) with a degree of asymmetry existing between the two, specifically that the B-branch cofactor exhibits greater distortion than that of the A-branch. With respect to the accessory pigments, the BChls are usually the least distorted of all the cofactors and 125 the bacteriopheophytins are considerably distorted. An important deviation from this pattern exists for the B-branch accessory BChl of $R$. sphaeroides in that this cofactor displays a far greater distortion than its A-branch counterpart such that its relative distortion is comparable to that of the B-branch special pair 130 cofactor. In both species, the conformations of the accessory BPhes are dominated by a $0.3-0.5 \AA \quad B_{1 u}$ distortion, with noticeable contributions from other modes. In terms of the cause of the distortions, that the special-pairs of both species are generally the most distorted cofactors indicates that their mutual 135 interaction is a crucial factor, whereas the differences within each accessory pair must be mediated by the apoprotein alone. The fact that we may attribute at least the increased distortion of the special-pair cofactors to their dimerization points to a new paradigm for its original formation (in addition to their excitonic 140 coupling), since this feature may be crucial to the overall ET process. Note that we have previously described such effects in small molecule bischlorin crystal structures. ${ }^{11}$

In light of the fact that increasing nonplanarity of tetrapyrroles has been linked with easier oxidation and more ${ }_{145}$ difficult reduction ${ }^{12}$ of the molecule, a point which has recently been exploited for heme redox modification via protein induced distortion in vitro, ${ }^{13}$ and that photosynthetic electron transfer is subject to the Marcus theory, the relative conformations of the BChl cofactors (above; in particular that the special-pairs are 150 always the most distorted and BA is always relatively planar) would seem to assist in charge-separation on the whole. The conformations may also contribute to the preference for L-branch electron transfer in both species. Mechanistically, the ET asymmetry is dominated by the initial charge transfer asymmetry 155 of the special-pair in $B$. viridis, which is echoed by the asymmetry in the extent of the nonplanar distortion exhibited by each half of the special-pair dimer in this species (Fig.'s. 3 and 4).
In $R$. sphaeroides the asymmetry of ET may begin with the conformational differences in the special-pair but could also be 160 enhanced by stabilization of the $\mathrm{SP}^{+} \mathrm{BA}^{-}$intermediate ${ }^{14}$ compared to the alternative $\mathrm{SP}^{+} \mathrm{BB}^{-}$intermediate, recalling the distortion of $\mathrm{BB}$ and that this will affect its hindered reduction. Note, that the rate of electron transfer is greater in $B$. viridis than in $R$. sphaeroides, ${ }^{15}$ which again correlates with the greater distortion 165 of the SP of the former (Fig. 4). The idea of the conformationally induced initial charge-transfer asymmetry (i.e. electron hole asymmetry at the special-pair) is supported by a recent study by Daviso et al. who concluded that the preferential localization of electron spin density to DL of the special-pair radical cation is 170 intrinsic to the dimer and “... [is] predominantly attributable to local conformational conservation...”. ${ }^{16}$ Thus, our hypothesis is consistent with, and aids in the understanding of, the observed properties of the ETC and provides a rationale for these properties that would be observed as intrinsic in keeping with 175 these recent computational findings. However, to this end, the idea of the easier oxidation of the more nonplanar macrocycle would in fact imply that it should be DM that exhibits the greater hole density.

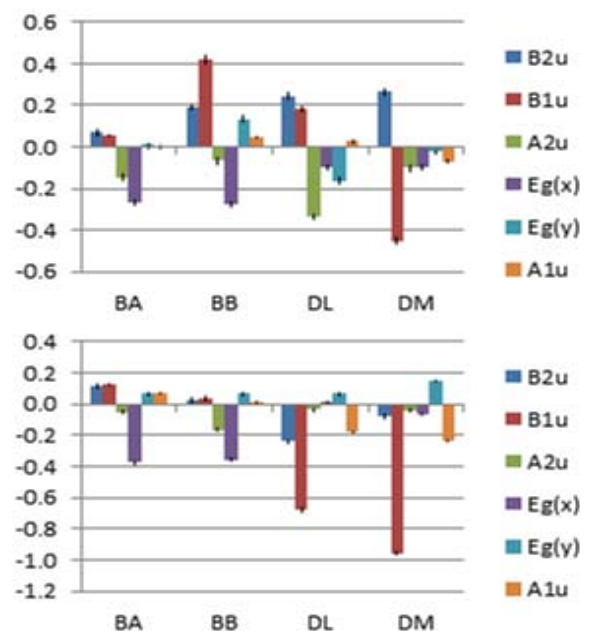

180 Fig. 3 Mean out-of-plane minimum basis NSDs of each ETC bacteriochlorin cofactor in R. sphaeroides (top) and B. viridis (bottom). $\mathrm{Y}$-axes in $\AA$; error bars indicate two standard errors. B. viridis, $\mathrm{n}=14$ for each cofactor; $R$. sphaeroides, $\mathrm{n}=32$ for each cofactor

On the other hand, not only is the understanding of ET aided 185 by conformational considerations but also that of photoprotection since it has recently been shown that increasing macrocycle distortion affects lower yields of singlet oxygen generation from the triplet state. ${ }^{17}$ This implies that the distortion of all but the advantageously planar cofactors (i.e. BA; see above) may be 190 beneficial to the RC in reducing the risk of oxidative damage. In addition, the apparently apoprotein modulated distortion of BB in $R$. sphaeroides may be a specific enhancement of its photoprotective role whilst the planar $\mathrm{BB}$ in $B$. viridis can account for the lack of efficient triplet quenching in this species. ${ }^{18}$ 195 This argument is also valid in terms of the triplet transfer to the carotenoid since the distortion induced red-shift may be what allows $\mathrm{BB}$ in $R$. sphaeroides to absorb the low energy triplet state of the SP in the first place. 


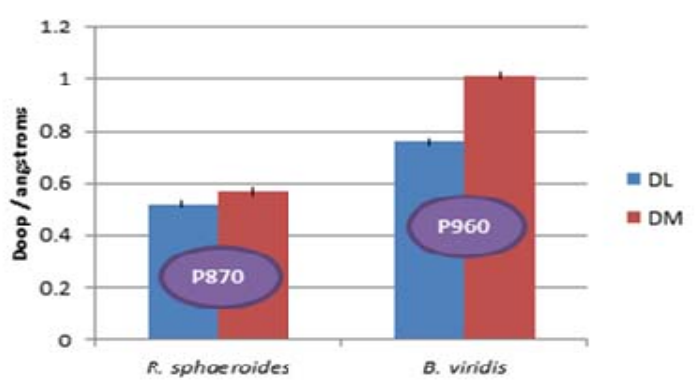

200 Fig. 4 Comparison of the total out-of-plane distortion $\left(D_{\text {oop }}\right)$ of the special-pairs from each species. $R$. sphaeroides, $\mathrm{n}=32$ (right) and $\mathrm{B}$. viridis, $\mathrm{n}=14$ (left) for each cofactor.

Further experimental observations and reaction center properties can also be explained with reference to the uncovered 205 conformations. Resonance Raman (RR) studies of RCs yield conformational information on the tetrapyrroles in solution. One such study of $R$. sphaeroides $^{19}$ indicated that of the four ETC BChls, one is conformationally unique and suggested that it is DL that is different. Our analysis shows that this cofactor is unique in 210 that its conformation is dominated by a relatively large $A_{2 u}$ distortion. Also, RR spectral studies indicate a more symmetric binding environment for the cofactors of P960 (the special-pair of $B$. viridis), yet electron paramagnetic resonance and electron nuclear double resonance studies show that CT is more 215 asymmetric in P960 than in P865. ${ }^{15}$ However, we can now see that the difference in overall nonplanarity is greater in the $B$. viridis special-pair. With this in mind the optical and nearinfrared absorption spectra of the bacteriochlorin cofactors in RCs of $B$. viridis and $R$. sphaeroides $^{20}$ may also be 220 conformationally modulated since the considerable red shift of the Qy band of P960 ${ }^{20 \mathrm{a}}$ compared to P865 ${ }^{15,20 \mathrm{~b}}$ (95 nm shift) can only in part be attributed to the different C8 substituents since the isolated compounds absorb at only 770 and $795 \mathrm{~nm}$, respectively, in solution ${ }^{21}$ ( $25 \mathrm{~nm}$ shift). However, the NSDs show P960 to be 225 far more distorted than P870; this type of red-shift is another known effect of porphyrin nonplanarity and more specifically, the large $\mathrm{B}_{1 \mathrm{u}(2)}$ distortion exhibited by P960 has been directly liked to such spectral shifts. ${ }^{2 b}$

We suggest that these statistics represent the actual 230 conformations found in the crystal and we provide coordinates for these cofactors. The conformations, together with the current understanding of the effects of nonplanarity, tie in with the observed physical properties of electron transfer in photosynthetic systems such as the species variant extent of the 235 bathochromic shift of the special-pair, the preference for Lbranch ET or the photoprotective action of BB in R. sphaeroides amongst others and therefore provide a new chemical rationale for understanding these processes. It is thus valid to suppose that the conformation contributes to some of these observables, 240 possibly in a deliberate rather than coincidental way; that nature has not only selected the primary structure of proteins to affect the desired protein conformations and cofactor spatial arrangements but also to induce particular cofactor conformations. The concept of conformational control of 245 tetrapyrrole cofactors can clearly be used to address questions of functional significance in the bacterial photosynthetic reaction center in a statistically reliable manner. This indicates the general validity this concept and indicates that it can be extended to hemes and other systems and serve as a model for structural 250 cofactor modulation in general.

This work was supported by Science Foundation Ireland (SFI P.I. 09/IN.1/B2650).

\section{Notes and references}

School of Chemistry, SFI Tetrapyrrole Laboratory, Trinity College 255 Dublin, Dublin 2, Ireland. Fax: +353 1896 8536; Tel:+353 1896 8537; E-mail: sengem@tcd.ie

† Electronic Supplementary Information (ESI) available: Explicit descriptions of the $\mathrm{R}$ packages and functions used and data configurations, detailed descriptions of the analytic process, all relevant 260 conformational data for all subsets and NSD bases (including coordinates), details of each PDB structure assessed and classified, and all individual cofactor NSDs. See DOI: 10.1039/b000000x/

1 (a) M. O. Senge, J. Photochem. Photobiol. B: Biol., 1992, 16, 3; (b) K. M. Barkigia, L. Chantranupong, K. M. Smith and J. Fajer, J. Am. 265 Chem. Soc., 1988, 110, 7566; (c) R. Huber, Eur. J. Biochem., 1990, 187, 283.

2 (a) M. O. Senge, in The Porphyrin Handook, Vol. 1 (K. M. Smith, K. M. Kadish, R. Guilard, eds.), Academic Press, San Diego, 2000, pp. 239; (b) R. E. Haddad, S. Gazeau, J. Pecaut, J.-C. Marchon, C. J. 270 Medforth and J. A. Shelnutt, J. Am. Chem. Soc., 2003, 125, 1253; (c) J. A. Shelnutt, X.-Z. Song, J.-G. Ma, S.-L. Jia, W. Jentzen and C. J. Medforth, Chem. Soc. Rev., 1998, 27, 31; (d) W. Jentzen, J. Ma and J. A. Shelnutt, Biophys. J., 1998, 74, 753.

3 (a) W. Jentzen, J. Ma and J. A. Shelnutt, Biophys. J., 1998, 74, 753; 275 (b) J. D. Hobbs and J. A. Shelnutt, J. Protein Chem., 1995, 14, 19.

4 (a) J. Deisenhofer,O. Epp, K. Miki, R. Huber and H. Michel, Nature, 1986, 318, 618; (b) G. Feher, J. P. Allen, M. Y. Okamura and D. C. Rees, Nature, 1989, 339, 111.

5 (a) J. Deisenhofer and H. Michel, Biosci. Rep., 2005, 24, 325; b) C. 280 R. Lancaster and H. Michel, Photosynth. Res., 1996, 48, 65.

6 M. E. Michel-Beyerle, M. Plato, J. Deisenhofer, H. Michel, M. Bixon and J. Jortner, Biochim. Biophys. Acta, 1988, 932, 52.

7 M. H. Stowell, T. M. McPhillips, D. C. Rees, S. M. Soltis, E. Abresch and G. Feher, Science, 1997, 276, 812.

2858 (a) W. R. Scheidt and C. A. Reed, Chem. Rev., 1981, 81, 543; (b) C. Kratky, R. Waditschatka, C. Angst, J. E. Johansen, J. C. Plaquevent, J. Schreiber and A. Eschenmoser, Helv. Chim. Acta, 1985, 68, 1312.

9 (a) W. Jentzen, M. C. Simpson, J. D. Hobbs, X. Song, T. Ema, N. Y. Nelson, C. J. Medforth, K. M. Smith and M. Veyrat, J. Am. Chem. $290 \quad$ Soc., 1995, 117, 11085; (b) W. Jentzen, X. Song and J. A. Shelnutt, J. Phys. Chem. B, 1997, 101, 1684.

10 (a) M. O. Senge and S. A. MacGowan, in Handbook of Porphyrin Science (K. M. Smith, K. M. Kadish, R. Guilard, Eds.), World Scientific/Imperial College Press, 2010; Vol. 13, pp. 253; (b) M. O. 295 Senge and K. M. Smith, Photochem. Photobiol., 1991, 54, 841; (c) C. E. Strouse, Proc. Natl. Acad. Sci. USA 1974, 71, 325.

11 M. O. Senge, W. W. Kalisch and K. Ruhlandt-Senge, Chem. Commun., 1996, 2149.

12 M. O. Senge, Chem. Commun., 2006, 243.

30013 C. Olea, J. Kuriyan and M. A. Marletta, J. Am. Chem. Soc., 2010, 132, 12794.

14 W. W. Parson and A. Warshel, in The Purple Phototrophic Bacteria (F. Daldal, C. N. Hunter, M. C. Thurnauer and J. T. Beatty, Eds.), Springer, Dordrecht, 2009, pp. 355.

30515 A. J. Hoff and J. Deisenhofer, Phys. Rep., 1997, 287, 1.

16 E. Daviso, S. Prakash, A. Alia, P. Gast, J. Neugebauer, G. Jeschke and J. Matysik, Proc. Natl. Acad. Sci. USA, 2009, 106, 22281.

17 B. Röder, M. Büchner, I. Rückmann and M. O. Senge, Photochem. Photobiol. Sci., 2010, 9, 1152.

31018 P. D. Laible, V. Chynwat, M. C. Thurnauer, M. Schiffer, D. K. Hanson and H. A. Frank, Biophys. J., 1998, 74, 2623.

19 V. Palaniappan, P. C. Martin, V. Chynwat, H. A. Frank and D. F. Bocian, J. Am. Chem. Soc., 1993, 115, 12035.

20 (a) J. Breton, Biochim. Biophys. Acta, 1985, 801, 235; (b) D. W. 315 Reed and B. Ke, J. Biol. Chem., 1973, 248, 3041.

21 J. Oelze, Meth. Microbiol., 1985, 18, 257. 


\section{GRAPHICAL ABSTRACT:}

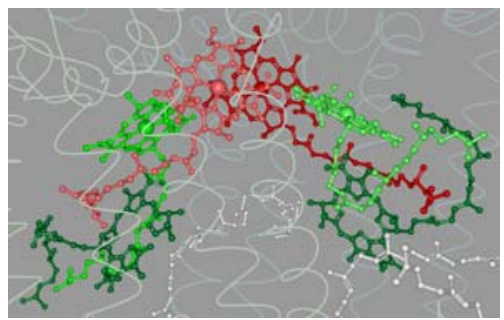

Each of the chemically identical cofactors of the electron transfer chain of purple bacteria adopts distinct conformations induced by the apoprotein. In describing how these conformational modifications

contribute to ETC function, a chemical rationale for their biofunctional utility is proposed in which the protein exerts conformational influence upon the cofactor to affect desired properties. This serves as a general model for the control of tetrapyrrole function in nature. 


\section{Supplementary Information}

Conformational control of cofactors in nature - Conservation of tetrapyrrole conformations in the photosynthetic reaction centers of purple bacteria

Stuart A. MacGowan and Mathias O. Senge

\section{Contents}

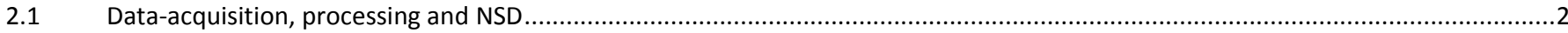

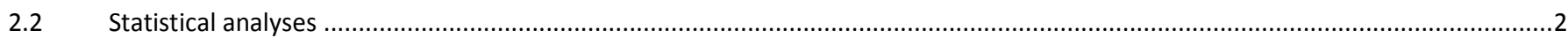

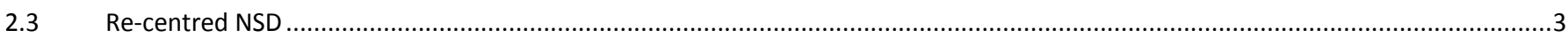

3 Preliminary analysis: Confirming experimental consistency with distinct cofactor conformations ........................................................

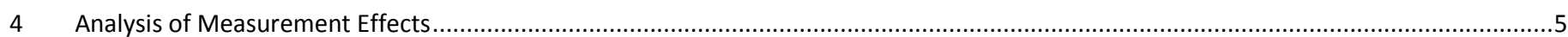

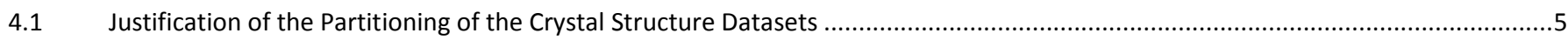

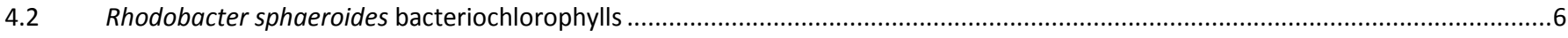

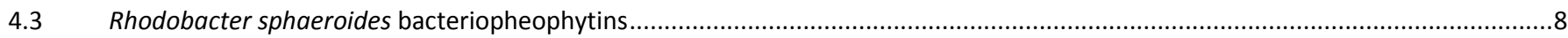

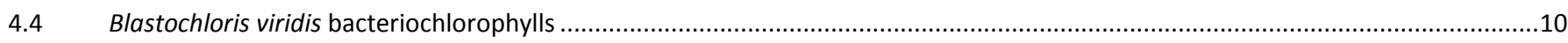

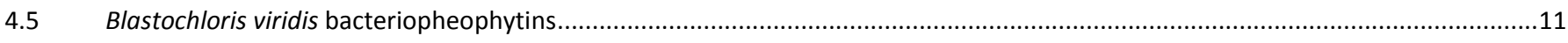

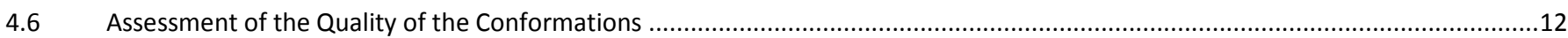

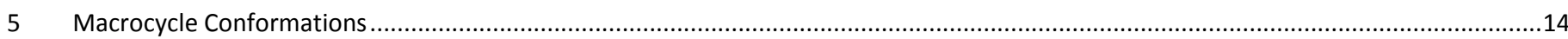

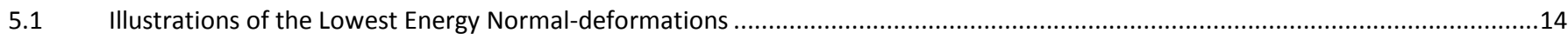

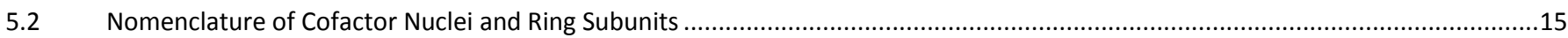

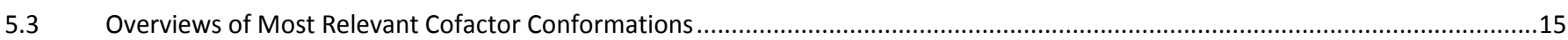

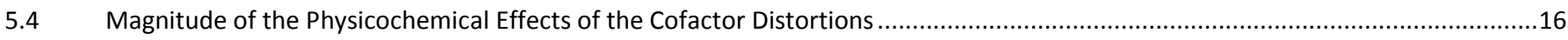



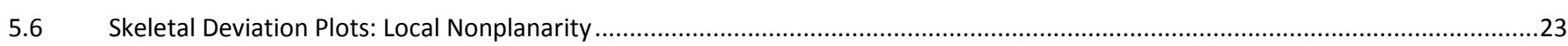

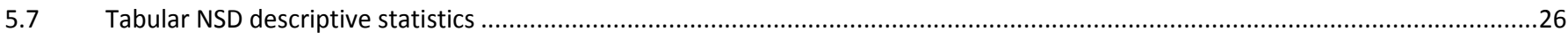

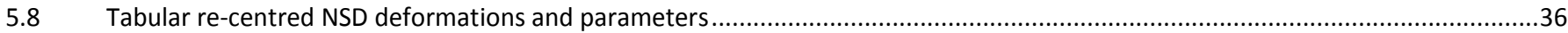





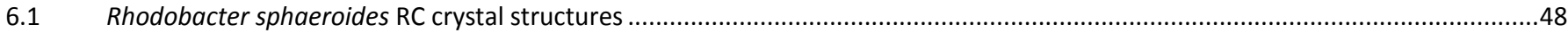

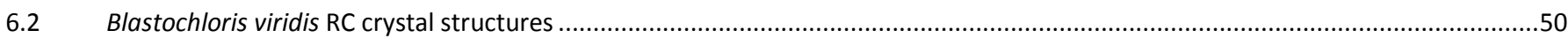

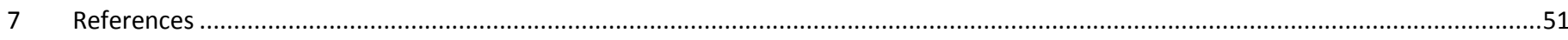

$8 \quad$ Cofactor NSDs

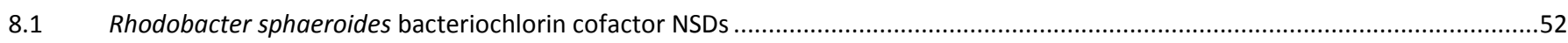

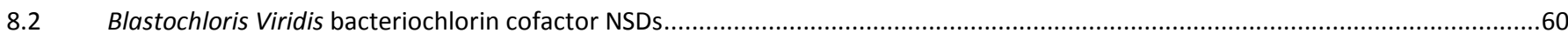

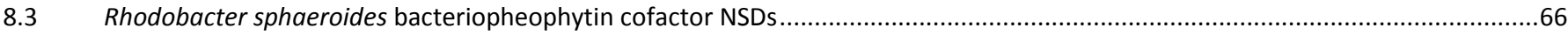






\section{Note on content}

Other than to provide the appropriate data and detailed experimental methods used by and produced from our analyses there are three major purposes of this supplement. The first is to show how we proved that almost the entire set of published reaction centre (RC) crystal structures was in agreement of with the idea of distinct conformations for each of the cofactor functionalities of the electron transfer chain (ETC). The second point is to provide explicit justification and to describe the process for/by which we have partitioned the reaction centre crystal structures, in particular the $R$. sphaeroides set, based on the resolved conformations of their ETC cofactors (Section 4). Finally, the third purpose is to illustrate the local nonplanar distortions of the cofactors (i.e. in terms of their individual atomic z-axis displacements; Section 5) and consequently, to demonstrate the specific adequacies of the NSD reduced basis sets that are cited in our work as a suitable vantage point from which to compare their conformations.

In brief, Section 2 contains the detailed experimental methodology that would be required to reproduce our work. A brief account of the analysis that confirmed that the crystal structure data were consistent with, and would yield, distinct cofactor conformations for each of the functionalities is relayed in Section 3 followed by the logic, rationale and analysis behind the crystal structure partitioning in Section 4 , including our argument for the selection of the best candidate crystal structure set to derive the mean cofactor conformations. Section 5 provides the full, quantitative conformational description of the conformations of each cofactor from each of the crystal structure subsets as well as the appropriate descriptive statistics. This includes the NSD data of the reduced basis deemed adequate for describing each particular conformation in both chart and tabular form; skeletal deviation plots of the cofactors from the most relevant subsets and the cofactor coordinates that we have derived. We have also provided brief descriptive conformational overviews of the cofactors we consider to be the most important from the set of crystal structures we determined to be most reliable, to place the data in context, and in addition we include a brief discussion of the magnitude of the potential physicochemical impact of the distortions. Finally, the PDB structures that were included in the study are presented in Section 6, references in Section 1 and the individual NSDs for each of the cofactors are given in Section 8.

\section{Experimental Methodology}

\subsection{Data-acquisition, processing and NSD}

The raw data, i.e. the cofactor atomic coordinates, were downloaded in batches by PDB ligand I.D. from the PDB Ligand Expo site (www.ligandexpo.rcsb.org; last accessed: $30^{\text {th }}$ April 2011), which is part of the RCSB PDB ${ }^{[1]}$ (www.pdb.org) project. Specifically, every BCL (BChl a), BCB ( $B C h l$ b), BPH (BPhe a) and BPB (BPhe b) found in the directory as of 18/10/2010 was downloaded in .ipdb format on that day; the BH1 cofactors (BPhe a of structures 2BNP, 2BNS and 2BOZ) were downloaded subsequently on 28/03/2011. Additionally, any cofactor structure that included alternate conformations was recompiled as two separate structures and the NSD of both were obtained. The PDB structures included are identified in Section 6.

The macrocycle of each cofactor was decomposed into deformations along its normal deformations by the procedure of normal-coordinate structural decomposition ${ }^{[2]}$ (NSD). The NSD computations were carried out using the online NSD Web Program ${ }^{[3]}$ with each cofactor oriented consistently, as described in the program's online documentation; the program automatically performs the NSD computation on any tetrapyrrole structure in PDB format. In order to speed up the tedious process of loading the coordinates, running the decomposition, reorienting the structure to obtain consistent signs (phases) for the normal-coordinates and storing the results, a short macro that automated much of the process (excepting operator verification of the cofactor orientation) was written and implemented in the AutoHotkey scripting language.

\subsection{Statistical analyses}

The preliminary analysis that is described in Section 3 involved the agglomerative hierarchical clustering (AHC) of each of the cofactors' minimum basis NSD results in order to discover whether or not the crystal structure data were consistent with the idea of distinct cofactor conformations for each of the functionalities. Thus, the AHC was performed on data matrices containing observations of the NSD minimum basis for each of the cofactors of a given species' with the XL Stat plugin for Microsoft Excel using the Euclidean distance measure to build the dissimilarity matrix and Ward's method for the agglomeration.

The collected NSD data were then treated so that each X-ray structure (PDB entry) was considered as an individual observation and the minimum basis NSD deformations of its cofactors the observables. In detail, the data was formed into an $m \times n$ matrix where $m$ is the number of crystal structures included and $n$, the number of variables, equals the number of cofactors included (i.e. four bacteriochlorophylls or two bacteriopheophytins) times the number of basis parameters used (i.e. $B 2 u_{-} m_{-} \mathrm{BA}^{*}$ is a distinct variable from $\mathrm{B} 2 \mathrm{u} \mathrm{m}_{-} \mathrm{BB}$ and there is no categorical variable for cofactor function); in the analyses of the experimental effects that follow, the 12 normal-coordinates of the minimum basis were used for each cofactor. The result of this approach was the potential to isolate so-called batch effects that are present from particular (sets of) structure determinations via variable-axis rotations from methods such as principal component and factor analysis (PCA and FA). The analyses presented in Section 4 were all performed on data matrices in this configuration.

\footnotetext{
${ }^{*}$ i.e. minimum basis $\mathrm{B} 2 \mathrm{u}$ displacement of cofactor $\mathrm{BA}$
} 
The kernel density estimations, Euclidean dissimilarities, FAs and the PCAs used were calculated in the R programing environment ${ }^{[4]}$ using the kde, daisy, factanal and prcomp functions in the ks, cluster and base libraries, respectively. The bandwidth parameters, $\mathrm{h}$, of the KDEs were chosen using the plug-in selector, executed with the hpi (or Hpi for bivariate KDEs) function also in the ks library. The decision as to the number of factors extracted in the factor analyses was made based on our initial exploratory analyses (Section 4). The data matrices were untransformed; Varimax rotation was performed in the FAs and the crystal structure scores were computed using the regression method.

Once the crystal structure set partitioning was complete, the mean (arithmetic) normal-coordinates were calculated to yield the average conformation of each of the cofactors in each subset of crystal structures (Section 5). Both the sample standard deviation (SD) and standard error of the mean (SE) are given where appropriate as measures of the sample variation; the SD represents the observed single experiment precision of each of the measured parameters whereas the SE should provide appropriate confidence intervals for the precision of the sample ${ }^{*}$.

\subsection{Re-centred NSD}

Included also in Section 5 are tabular listings of NSD data that we have termed as the 're-centred NSD' (Section 5.7). These data were obtained by performing the NSD computation on coordinates that were obtained from the statistical mean complete basis NSD for each cofactor of each subset (coordinates which are approximately equivalent to averaged atomic coordinates). For example, the re-centred NSD of cofactor DL from subset RBC4 was obtained by converting the corresponding statistical average NSD deformations of the complete basis to Cartesian coordinates and then performing the NSD computation on this structure. The reason for doing this was twofold; to obtain the Doop and $\delta o o p$ parameters for the mean conformation and also to ensure that the mean NSD deformations were representative of the NSD of the mean conformation. This latter point was considered a prudent check since the extended and complete basis sets are not completely orthogonal ${ }^{[2 \mathrm{~b}]}$ although we did not expect any substantial deviation owing to the relatively small dot products of the normal-deformation vectors ${ }^{[2 \mathrm{~b}]}$ and our own analysis of the numerical stability of NSD based on the condition numbers of the basis matrices. Whilst the complete basis re-centred molecular conformations are precisely the same as those given by the mean results the NSD description of this conformation may have been different due to the nonorthogonality of the complete basis.

\section{Preliminary analysis: Confirming experimental consistency with distinct cofactor conformations}

Preliminary analysis of the collected data was performed using agglomerative hierarchical clustering (for AHC details see Section 2.2) to identify structures bearing similar conformations, in terms of the in- and out-of-plane distortions of the minimum basis.

The Blastochloris viridis AHC (Figure 1) shows that there were six clusters of cofactors bearing similar conformations (including one cluster of a single structure, indicating its relative uniqueness) each of which contained predominantly only structures of one particular ETC functionality. Specifically, the cluster pattern demonstrates that the DM and DL cofactors (i.e. each half of the special-pair) are conformationally distinct from the accessory monomers and also from each other (although they are related relative to the rest as seen by the position of their cluster union in the dendrogram; top right, Figure 1). It is also seen that the first accessory cofactors (BA and BB) form a distinct group but are on the whole not distinguishable from each other whilst the second accessories (ФA and $Ф B$ ) form two groups, each predominantly composed of one of either $\Phi$ A or $\Phi B$, but not exclusively, and that these clusters are also closely related. The cluster that consists of a single observation was identified as a DM cofactor from a 'hetero-dimer' mutant structure where the DM bacteriochlorophyll had been replaced with a bacteriopheophytin; but note its proximity to the main DM cluster. The OOP mean conformations of each of the cofactors that were correctly classified are also displayed with these results (bottom left, Figure 1).

Similar to the above, the $R$. sphaeroides AHC proved that there were distinct conformations for each of the functionalities in this species too (Figure 2), the main difference being the presence of a cluster that predominantly contained the BB cofactors and the closeness of the DL group to that of the BA's (implying their conformational similarity). In addition, whilst there were two groups for the accessory bacteriopheophytins, the cluster membership for each group was split equally between $\Phi$ As and $\Phi B$ s. Also note that the BA group not only contained the vast majority of the BA cofactors, but also a significant number of the other bacteriochlorin functionalities (i.e. BB, DM, and DL; bottom right, Figure 2).

\footnotetext{
* Whilst we do not assert that the samples themselves are normally distributed we do invoke the Central Limit Theorem to state that the sample mean should be so and hence the SE provides approximate $95 \%$ confidence intervals for the means. The SDs may provide a useful test to see whether we have accounted for all of the systematic variation of the data by comparing them to the theoretical precision of a single experiment. However, we have not attempted this here as this calculation, to our knowledge, is not trivial. To do so, one would need to know the approximate experimental error in each NSD at a given resolution and would need to take into account: the theoretical mean atomic positional error, a reduced dimensionality component owing to separation of the in- and out-of-plane coordinates (already performed by Jentzen et $a l)$, the error in the calculation of the mean plane, the effect of restraints on these errors and possibly, owing to the random distribution of positional error, its probabilistic propagation into each mode given the nodal (phase change) patterns of the different normaldeformations. In addition to this later point, should probabilistic concerns be minor, at the very least one would need to know entrywise condition numbers for the NSD calculations.
} 

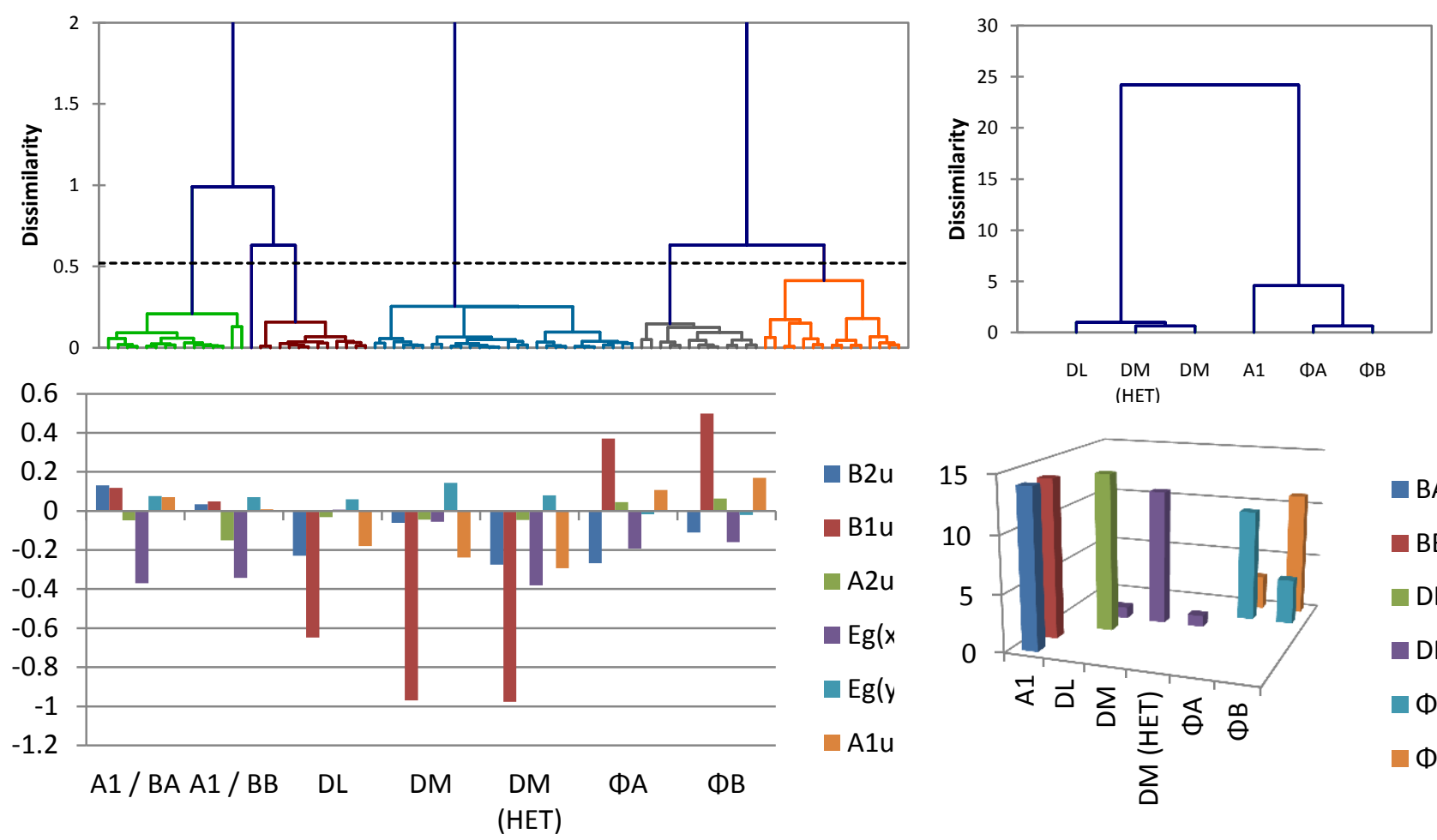

B2u

- B1u

A2u

- $\mathrm{Eg}(\mathrm{X}$

Eg(y

- A1u

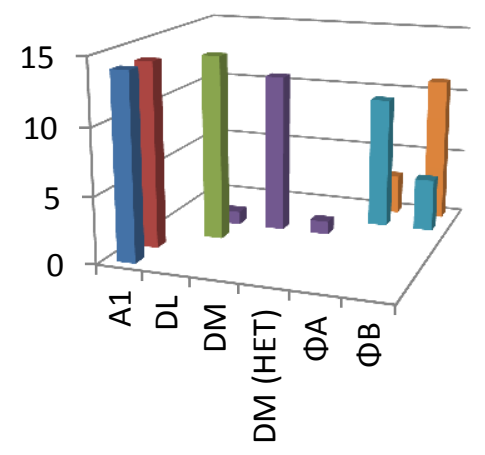

- BA

BB

DL

DM

- ФA

ФB

Figure 1: Initial AHC of B. viridis data. Cluster dendrograms (top) showing the clusters that predominantly contain DL (green), DM (brown), A1 (blue), ФA (grey) and ФB (orange) cofactors and their relationship; dissimilarity is given by Wards' criterion. Cluster assignment by function (bottom right); the axis labels indicate the cluster as seen in the dendrogram and the coloured bars enumerate how many cofactors of that functionality were present in the cluster. Mean minimum basis distortions of the correctly classified cofactors (bottom left; i.e. of cofactors that were present in the cluster pertaining to their functionality). $n=84$, within class variance $=0.053(12.59 \%)$, between class variance $=0.371(87.41 \%)$.
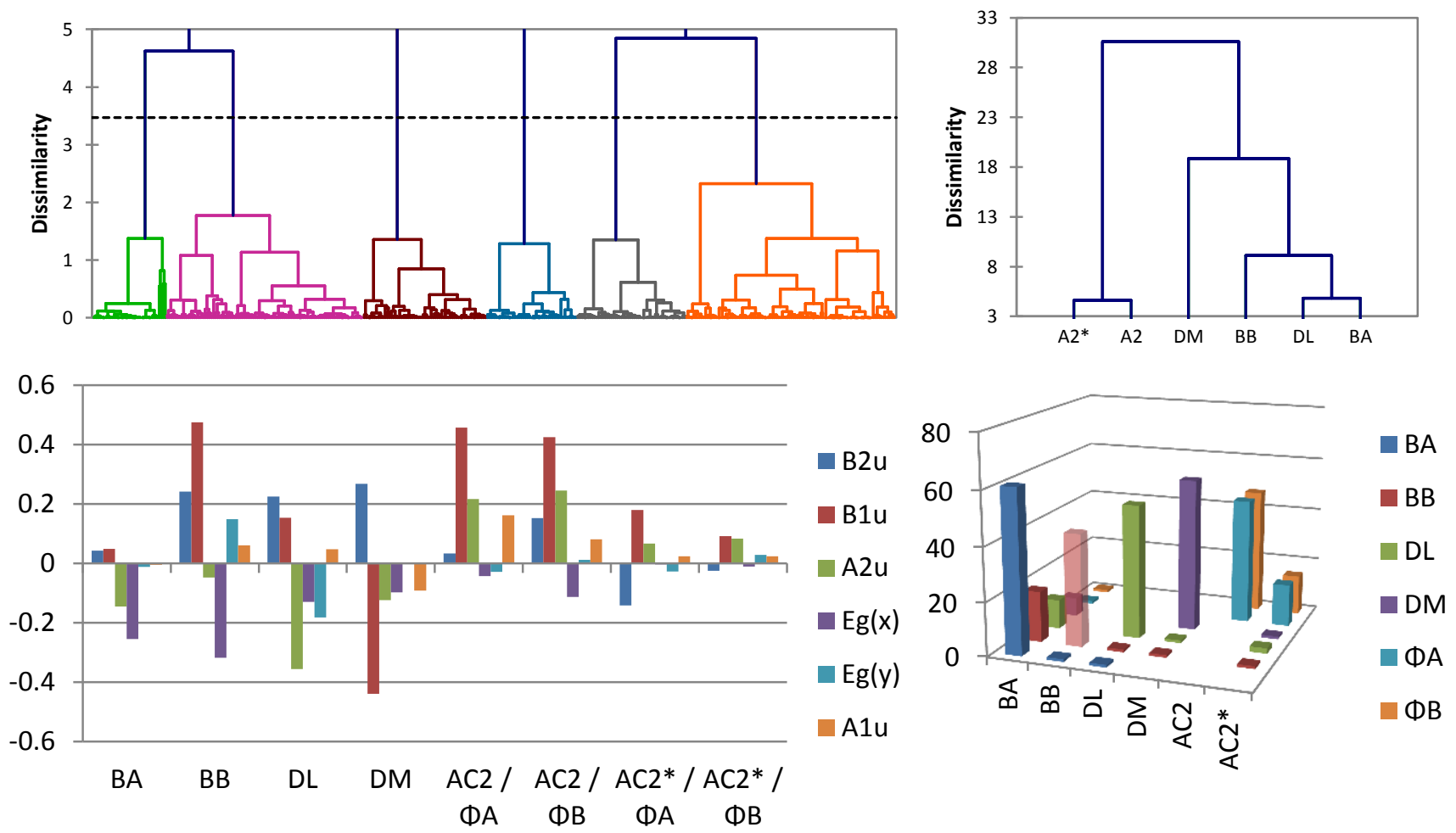

Figure 2: Initial AHC of $R$. sphaeroides data. Cluster dendrograms (top) showing the clusters that predominantly contain DL (grey), DM (brown), BA (orange), BB (blue), and the two clusters containing the bacteriopheophytin, AC2 (pink) and AC2* (green) cofactors and their relationship; dissimilarity is given by Wards' criterion. Cluster assignment by function (bottom right); the axis labels indicate the cluster as seen in the dendrogram and the coloured bars enumerate how many cofactors of that functionality were present in the cluster. Mean minimum basis distortions of the correctly classified cofactors (bottom left; i.e. of cofactors that were present in the cluster pertaining to their functionality). $n=383$, within class variance $=0.104(37.04 \%)$ and between class variance $=0.177(62.96 \%)$. 
The explanation of these features rest in the conformations of the functionalities, as captured by the mean deformations (bottom left, Figures 1 and 2). Thus, in $B$. viridis the BAs and BBs are similar in their relative absences of the B1u (ruffle) deformation and the DLs and DMs differ mostly in the extent, rather than make-up, of their distortions (DM being the most distorted cofactor). In contrast, the $R$. sphaeroides $\mathrm{BB}$ cofactor exhibits a predominant and considerable B1 $\mathrm{u}$ distortion and its DL is very different from DM owing to a phase change of the B1 $\mathrm{U}$ mode and the predominance of a large $\mathrm{A} 2 \mathrm{u}$ (dome) distortion. Furthermore, owing to the relative planarity of the BA type in $R$. sphaeroides (which appears to be a conserved feature between the species) it should be clear that the 'misclassified' (i.e. non BA cofactors in this group) display similar, near planar conformations and it should also be noted the AC2* set contains relatively planar $\Phi$ As and $\Phi B s$. Finally, whilst in both species the bacteriopheophytins are quite distinct from the bacteriochlorin cofactors (particularly in $R$. sphaeroides), neither species contains completely differentiable second accessories (in terms of A- / B- branch differentiation).

The interpretation of these results goes as far as to say that our initial hypothesis was somewhat validated; in that the protein scaffold imposes a rigid conformation on each of the cofactors, illustrated by the overall correct grouping of the cofactors' conformations by functionality. However, a critique of these observations would in fact point out that the only conclusion that can be definitively drawn is that the majority of the data is consistent with this hypothesis. In fact, on closer inspection we find that, for example, the PDB structures that contain planar DMs (a significantly nonplanar cofactor in most of the structures) report planar conformations for all the cofactors. Thus, a minority of structures are actually consistent with all of the cofactors having planar conformations. Of course, one could say simply that these are anomalous given their lesser numbers, but this would not be proper scientific process.

Fortunately, these types of results were anticipated at the outset of the investigation owing to the fact that the process of crystal structure determination is not always just a measurement of molecular dimensions; it often uses chemical knowledge to fill in the gaps that are present from lower resolution studies such as these. With this in mind, the extent of any aromatic planarity restraints, or in the extreme case, the presence of any such constraints may affect the observations of the planar macrocycles. Alternatively, it may be simply that the crystallographer felt that owing to the low resolution of his study, he could not comment accurately on the detailed conformation of the cofactors, and solved this part of the structure using rigid-body refinement of planar macrocycles.

There was one other such systematic variation between some of the structures in the $R$. sphaeroides data that became apparent during the initial AHCs. Notice in the dendrogram (top left, Figure 2) that there is a systematic pattern within each of the clusters, which indicates that there is more order to the data than simply the distinct conformations of the functionalities. Since the complete description of this variation via the more detailed AHCs that were carried out is laborious and difficult to follow, it suffices to say that following a similar method we discovered a subset of structures that differed in the extent of their nonplanarity and exhibited an unusual in-plane conformation and then to move on to the mode of analysis which uncovered the patterns of experimental origin.

\section{Analysis of Measurement Effects}

\subsection{Justification of the Partitioning of the Crystal Structure Datasets}

The significance of the following classification of the X-ray crystal structures of the bacterial electron transfer chains under study, based on the observed conformations of their tetrapyrrole cofactors rests on only one simple hypothesis:



That is to say that the cofactors are sufficiently removed from one another that the observed systematic differences between all of the cofactors of a given structure and another's geometries arise because of systematic differences between the measurements and not because of systematic differences between their conformations. As an example, consider two hypothetical crystal structures $A$ and $B$. If the four bacteriochlorophyll cofactors of A exhibit consistently and quantitatively precise increased nonplanarity over those of $B$, such that the relative difference between the conformations of the cofactors within each structure is maintained then we may draw two conclusions:

1) The relative differences of this conformational parameter between the specific cofactor functionalities has been measured precisely by both experiments

2) In the absence of any chemical or physical explanation of this difference the effect must arise from a systematic difference between the measurements of the two samples.

This is an ideal example (with obvious conclusions); in reality due to the random distribution of experimental error it is highly unlikely that such differences would obtain quantitative precision. However, this does not present a problem since our dataset (particularly of structures of $R$. sphaeroides) is moderately large and our applications of principal component analysis (PCA) and, in particular, factor analysis (FA) are able to resolve such features over this noise. 
With reference to the possible exceptions stated in this hypothesis, it was assumed that the experimentally correlated systematic differences would overwhelm such features. For instance, it is reasonable to suppose that in the charge-separated state a change of the conformations of the $\mathrm{P}^{+}$and $\Phi \mathrm{A}^{-}$cofactors, relative to their ground-state neutral counterparts would occur, however subtle. This would manifest itself as a systematic difference between structures in these two states and may be recoverable as a factor in the FA. We have not, however, found such a factor and though its definitive exclusion would require an even deeper statistical analysis, it is likely that the combination of the slightness of the change and the relatively low resolutions of the structures would make a feature such as this presently unobservable. Also, point mutations close to any of the cofactors may sometimes affect a change in their conformation, as has recently been demonstrated experimentally and were these perturbations larger than the experimental error one would expect systematic differences to be observed between structures of different mutations. However, again we have not been able to detect such features but if they are present there is no reason for them to have an effect on our results stated in this work.

However, on the other hand, it cannot be asserted with absolute authority that the inter-crystal structure set differences to be described do not represent real conformational differences; we consider this is quite unlikely (see also Section 4.6). In any case, the structure subsets should not be treated together since in such a situation that there was a chemical or physical reason for the coupling of the conformations so that the differences were actual (that we have not picked up on), these differences are so large compared to those within each set that they would have to be taken as an indication that the cofactors adopted multiple discrete conformations and so they would need to be treated separately anyway. It is for this reason that we have provided details of all the crystal structure subsets in this supplement.

\subsection{Rhodobacter sphaeroides bacteriochlorophylls}

Our exploratory analyses indicated that there were systematic differences between sets of structures as well as the inter-cofactor conformational data structure that was our target. These structure solution based differences are 'batch effects' of the crystal structure determinations and knowledge of their precise identity, character, elemental composition and origin were required to ensure a sound analysis. We identified four distinct sets in the $R$. sphaeroides bacteriochlorophyll dataset:

1. Structures that did not exhibit reasonable experimental variation (RBC1; $\mathrm{n}=9$ )

2. Structures that resolved the cofactors as planar or near planar $(\mathbf{R B C} 2 ; n=18)$

3. Structures with unusual in-plane conformations $(R B C 3 ; n=16)$

4. Structures that appear most reliable $(\mathbf{R B C} 4 ; \mathrm{n}=32)$

The purpose of this supplementary section is to provide the necessary information and derivation required to partition the data $(R$. sphaeroides bacteriochlorophyll set) into these clusters and also the (publically available) material required for their explanation.

\subsubsection{Tied structures}

The inter-structure Euclidean distance plot (Figure 3) shows a distinct group of outliers with near zero dissimilarity; it is clear that they originate from a different statistical distribution since they are present only after a distinct discontinuity of the empirical cumulative distribution of this parameter. Close inspection of the source of these outliers shows that they are closely related in terms of their experimental circumstance (i.e. they were measured at the same time by the same group (see Section 6, Table 41) and share a common starting model. We therefore interpret this subset as erroneous due to lack of appropriate experimental variability as they are replicas and do not represent independent measurements. The structures identified as such are labelled RBC1 (Section 6.1, Table 41) whilst the mean conformations exhibited by each cofactor may be found in Section 5.4.1, Figure 12.


Figure 3: Plot of inter-structure Euclidean distances of minimum basis from all $R$. sphaeroides bacteriochlorophyll cofactors. 


\subsubsection{Factor analysis: Identification of Crystal Structure Experimental Variations}

Subsets RBC2 (planar / near planar bacteriochlorophylls) and RBC3 (contracted bacteriochlorophylls) were identified in our original analyses through a combination of graphical and numerical exploratory measures. However, we found that we could express these findings most concisely in the form of an exploratory factor analysis (EFA). The optimal EFA was found to consist of two Varimax rotated factors, accounting for $44.1 \%$ of the total variance ( 22.6 and $21.5 \%$ each, respectively), extracted from the structure bacteriochlorophyll minimum basis data matrix. The factor loadings (Table 1) demonstrate that Factor 1 (F1) is mostly related to the nonplanarity exhibited by the cofactors in the structure determination whilst Factor 2 (F2) accounts for the observed structures with unusual in-plane conformations. With these loadings in mind, the apparent clusters observable in the plot of the structure scores (Figure 4) are readily identified, i.e. structures with a large negative score on F2 (mostly bottom left in Figure 4) exhibit unusually contracted macrocycles together with enhanced nonplanarity, particularly along the higher energy normal-modes (illustrated by $\delta o o p$ loading) whilst those crystal structures with a positive F1 value have resolved near planar cofactors. We therefore identify the bottom left cluster as RBC3 and the top and middle right clusters, which exhibit near planar and minimally nonplanar conformations (respectively) as corresponding to RBC2 (with descriptive statistics given separately as RBC2a and -2b, respectively, in Section 5.4.1) The remaining cluster (top left), which contains the highest proportion of structures of a single cluster, is identified as RBC4 which contains structures that exhibit significant nonplanarity with in-plane deformations akin to those of isolated related compounds. Also, note that we have treated structures that fall significantly outwith the level 25 regions of the KDE contours as outliers (OLs; see Section 6.1 for affected structures).

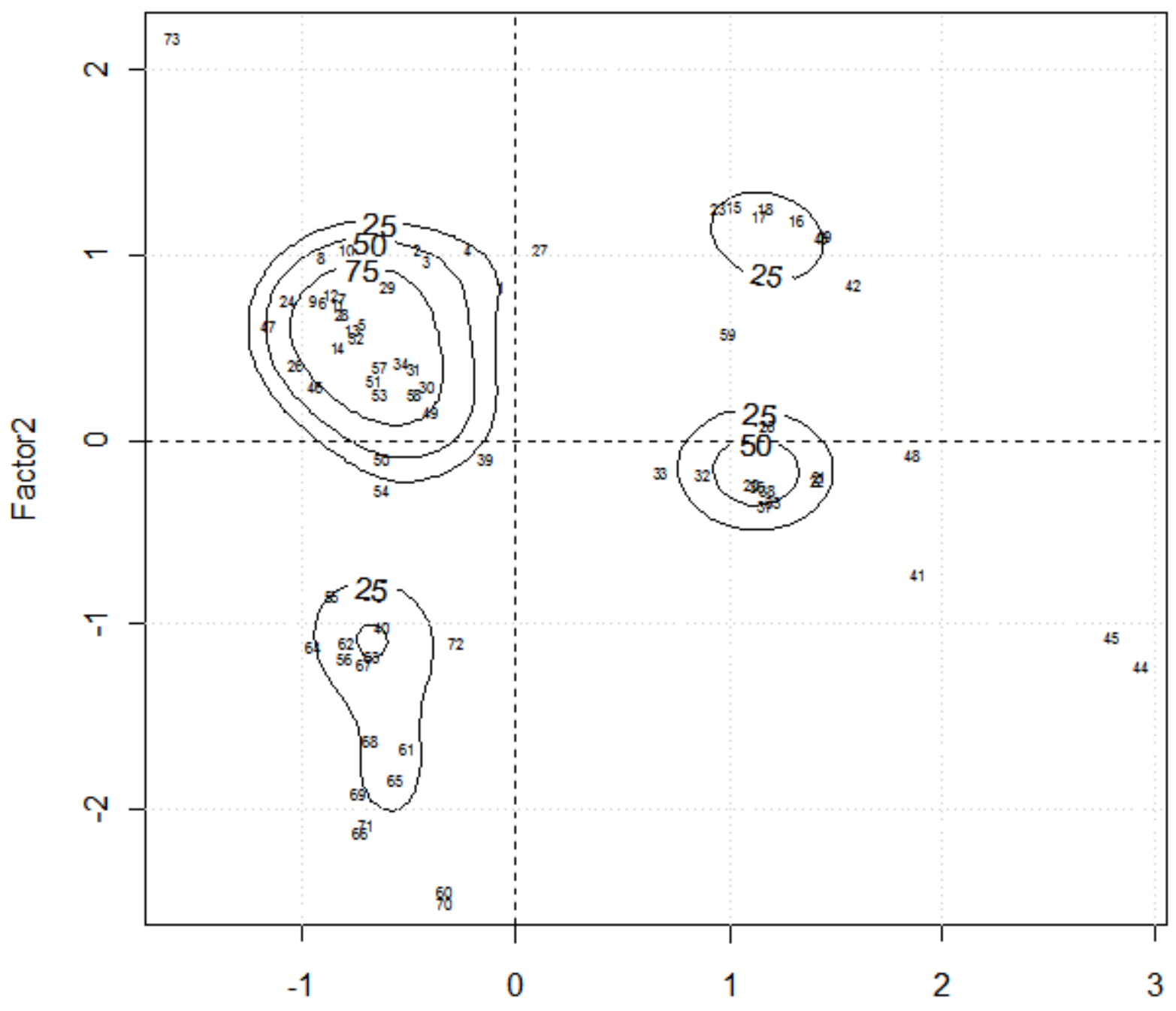

Factor1

Figure 4: Plot of the structure scores, on the Varimax rotated factors, computed by the regression method with kernel density contours overlaid (bandwidth matrix computed using the plug-in selector). Structure key in Section 6.1. 


\begin{tabular}{|c|c|c|c|c|c|c|c|c|}
\hline & $\mathrm{DM}$ & $\mathrm{DL}$ & BB & BA & $\mathrm{DM}$ & $\mathrm{DL}$ & BB & $\mathrm{BA}$ \\
\hline \multicolumn{5}{|c|}{ Doop } & \multicolumn{4}{|c|}{ Dip } \\
\hline $\mathrm{F} 1$ & -0.857 & -0.844 & -0.823 & -0.767 & 0.301 & 0.082 & -0.067 & -0.011 \\
\hline F2 & -0.406 & -0.428 & -0.411 & -0.457 & 0.290 & 0.522 & 0.264 & 0.263 \\
\hline \multicolumn{5}{|c|}{ Soop } & \multicolumn{4}{|c|}{$\delta i p$} \\
\hline $\mathrm{F} 1$ & -0.435 & -0.543 & -0.419 & -0.512 & 0.851 & 0.798 & 0.687 & 0.731 \\
\hline F2 & -0.743 & -0.752 & -0.707 & -0.733 & -0.210 & -0.246 & -0.013 & -0.072 \\
\hline \multicolumn{5}{|c|}{$B 2 u$} & \multicolumn{4}{|c|}{$B 2 g$} \\
\hline F1 & -0.697 & -0.526 & -0.712 & -0.421 & 0.496 & 0.243 & 0.084 & -0.115 \\
\hline F2 & -0.319 & -0.128 & -0.273 & 0.066 & -0.297 & -0.410 & -0.586 & -0.382 \\
\hline \multicolumn{5}{|c|}{$B 1 u$} & \multicolumn{4}{|c|}{$B 1 g$} \\
\hline $\mathrm{F} 1$ & 0.837 & -0.618 & -0.797 & -0.295 & 0.196 & 0.194 & 0.359 & 0.372 \\
\hline F2 & 0.341 & 0.225 & -0.357 & 0.178 & 0.230 & 0.262 & 0.299 & 0.378 \\
\hline \multicolumn{5}{|c|}{$A 2 u$} & \multicolumn{4}{|c|}{$E u(x)$} \\
\hline F1 & 0.389 & 0.784 & -0.100 & 0.240 & -0.382 & -0.191 & -0.338 & -0.273 \\
\hline F2 & 0.341 & 0.419 & -0.105 & 0.207 & -0.100 & -0.209 & -0.286 & -0.048 \\
\hline \multicolumn{5}{|c|}{$E g(x)$} & \multicolumn{4}{|c|}{$E u(y)$} \\
\hline F1 & 0.467 & 0.343 & 0.745 & 0.735 & 0.443 & 0.167 & 0.551 & -0.014 \\
\hline F2 & 0.286 & 0.645 & 0.496 & 0.563 & 0.010 & 0.338 & 0.228 & -0.172 \\
\hline \multicolumn{5}{|c|}{$E g(y)$} & \multicolumn{4}{|c|}{$A 1 g$} \\
\hline F1 & 0.272 & 0.597 & -0.397 & 0.243 & 0.028 & 0.136 & 0.071 & 0.063 \\
\hline F2 & -0.224 & 0.479 & -0.244 & 0.390 & 0.807 & 0.824 & 0.904 & 0.797 \\
\hline \multicolumn{5}{|c|}{$A 1 u$} & \multicolumn{4}{|c|}{$A 2 g$} \\
\hline F1 & 0.472 & -0.477 & -0.335 & -0.164 & 0.087 & -0.083 & 0.048 & 0.022 \\
\hline F2 & 0.550 & -0.584 & -0.432 & 0.098 & 0.863 & 0.771 & 0.875 & 0.838 \\
\hline
\end{tabular}

\subsection{Rhodobacter sphaeroides bacteriopheophytins}

Given the nature of subsets identified within the $R$. sphaeroides bacteriochlorophyll data, it was expected that similar groups should arise within that of the bacteriopheophytins, in particular, subsets RBC1 and RBC2. Thus, the same analytical methodology (inter-structure Euclidean distance plots and two-factor EFA) was applied here and confirmed the presence and identity of the analogous subsets:

1. Structures that did not exhibit reasonable experimental variation (RBP1; $\mathrm{n}=10$ )

2. Structures that resolved the cofactors as planar or near planar (RBP2; $n=16)$

3. A set consisting mostly of the structures found in RBC3 (RBP3; $n=17$ )

4. A set consisting mostly of the structures found in RBC4 (RBP4; $n=16)$

In addition, in this case, the EFA defined another subset (RBP5; $\mathrm{n}=10$ ) that had no explicit counterpart in the $R$. sphaeroides bacteriochlorophyll dataset (but does appear to be fairly related to RBC4).

\subsubsection{Tied Structures}

The inter-structure Euclidean distance plot based on the Rhodobacter bacteriopheophytin data (Figure 5) shows the presence of a subset of structures that exhibit effectively no experimental variation. As would be expected, included in this set are the structures from its counterpart in the bacteriochlorophyll data, RBC1 (see Section 6.1). However, in this case there is one extra structure exhibiting the lack of any experimental variance, namely PDB ID: 1QOV. 

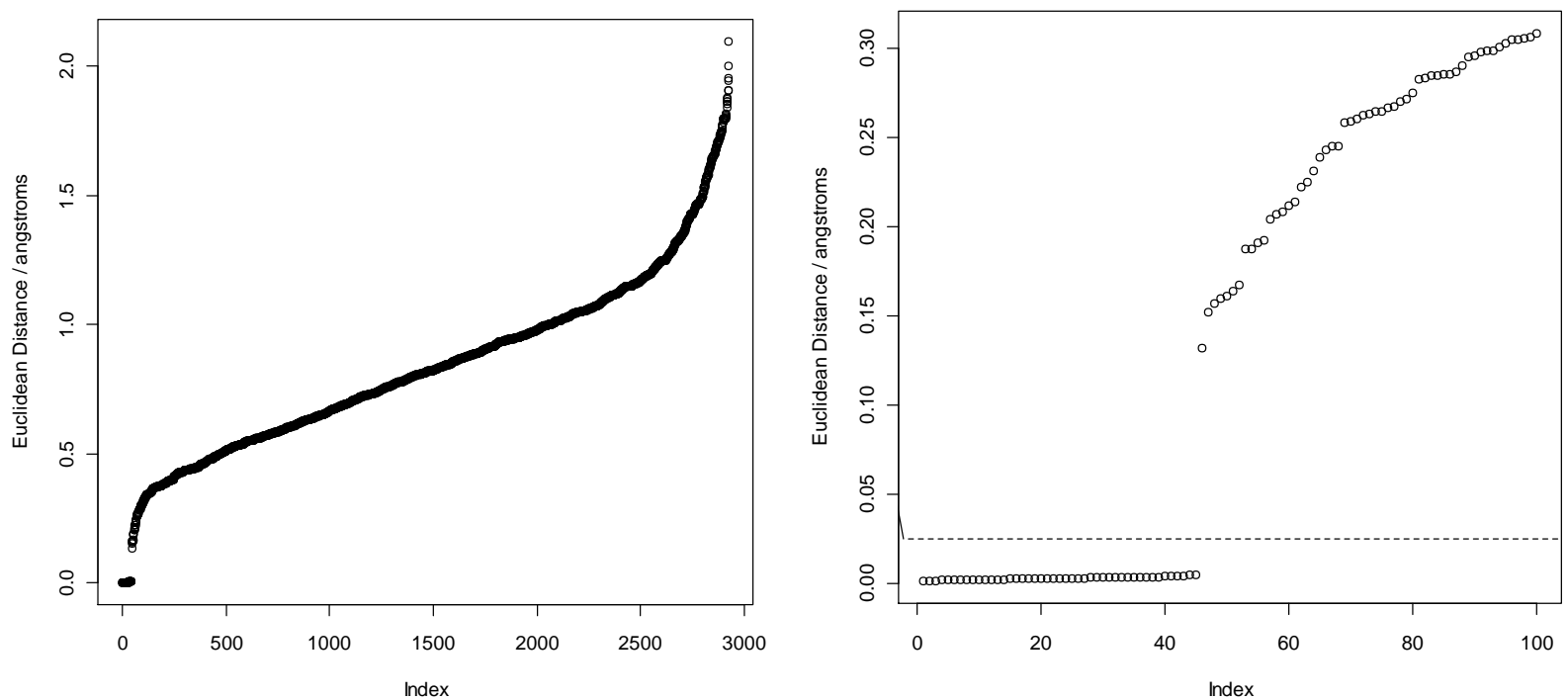

Figure 5: Plot of inter-structure Euclidean distances of minimum basis from all Rhodobacter bacteriopheophytin cofactors.

\subsubsection{Factor Analysis}

Factor 1 (26.2\% total variance; F1) of the Rhodobacter bacteriopheophytin EFA (Figure 6 and Table 2) is seen to correlate principally with the observed nonplanarity of the bacteriopheophytins and serves to differentiate, in order of increasing nonplanarity, subsets RBP2, -3 and -4. The nature of RBP2 is a somewhat trivial matter; it is the bacteriopheophytin counterpart of RBC2 in both composition (PDB structures; see Section 6.1) and conformational structure (planar cofactors). The differentiation of RBP3 and $\mathbf{- 4}$, however, is more interesting since these sets are composed of the same structures that are found in RBC3 and $\mathbf{- 4}$ (Section 6.1) and whilst in this case they are not separated on account of an in-plane A1g contraction, they are separated by numerous in- and out-of-plane parameters (Table 2), such that the difference between RBP3 and -4 is quite similar, qualitatively (compare NSDs in Section 5.4.3), as that of RBC3 and -4. This is particularly apparent from the large

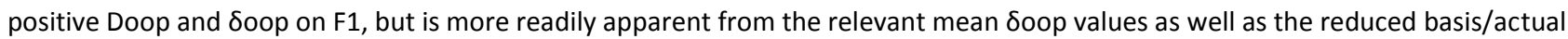
conformation comparisons in Sections 5.4 and 5.5.1. Note also that factor 2 accounts for $14.1 \%$ of the total variance and that the total variance accounted for by the two factors is $40.3 \%$, which is very similar to the variance accounted for by the two factors in the corresponding bacteriochlorophyll analysis (44.1\%) which confirms their relationship further. That $F 2$ in this analysis accounts for less variance than its counterpart in the bacteriochlorophyll set is readily acceptable since by comparison of the two analyses (below, Figure 6 and Section 4.2.2, Figure 4) shows that in this analysis RBP3 is differentiated from RBP4 by a combination of F2 and F1, as opposed to the differentiation of the related bacteriochlorophyll crystal structure sets (RBC3 and RBC4) by F2 alone. 


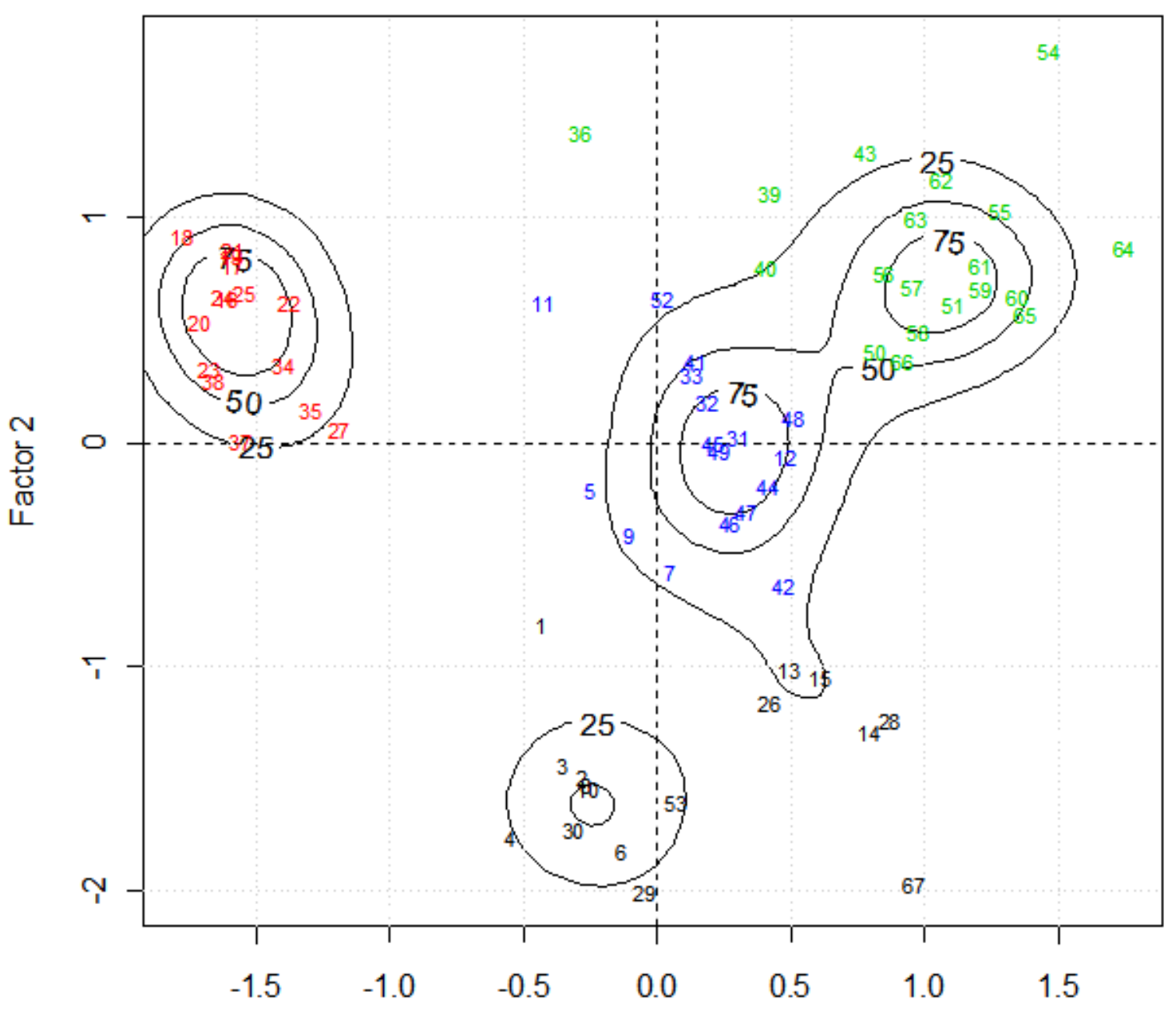

Factor 1

Figure 6: Plot of the structure scores, on the Varimax rotated factors, computed by the regression method with kernel density contours overlaid (bandwidth matrix computed using the plug-in selector). Structure key in Section 6.1; colouring for illustrative purposes only.

Table 2: Loadings of the original variables onto the Varimax rotated factors. Loadings in highest $\mathbf{2 5 \%}$ for each factor are marked in bold italic.

\begin{tabular}{|c|c|c|c|c|c|c|c|c|c|}
\hline & \multicolumn{2}{|c|}{ Factor 1} & \multicolumn{2}{|c|}{ Factor 2} & & \multicolumn{2}{|c|}{ Factor 1} & \multicolumn{2}{|c|}{ Factor 2} \\
\hline & $\Phi A$ & $\Phi B$ & $\Phi A$ & $\Phi B$ & & $\Phi A$ & $\Phi B$ & $\Phi A$ & $\Phi B$ \\
\hline Doop & 0.842 & 0.921 & -0.037 & -0.087 & Dip & 0.291 & 0.187 & 0.583 & 0.869 \\
\hline$\delta o o p$ & 0.870 & 0.830 & 0.213 & 0.285 & $\delta i p$ & 0.008 & 0.044 & 0.459 & 0.670 \\
\hline$B 2 u$ & 0.220 & 0.596 & 0.032 & 0.155 & $B 2 g$ & 0.213 & 0.302 & 0.681 & 0.579 \\
\hline$B 1 u$ & 0.741 & 0.663 & -0.205 & -0.279 & $B 1 g$ & -0.277 & -0.105 & -0.392 & -0.732 \\
\hline$A 2 u$ & 0.758 & 0.797 & 0.030 & -0.040 & $E u(x)$ & -0.389 & 0.019 & -0.142 & -0.093 \\
\hline$E g(x)$ & -0.287 & -0.438 & -0.200 & -0.241 & $E u(y)$ & -0.047 & -0.176 & -0.374 & -0.315 \\
\hline$E g(y)$ & -0.155 & 0.056 & 0.122 & -0.234 & $A 1 g$ & -0.018 & -0.036 & 0.117 & 0.562 \\
\hline$A 1 u$ & 0.730 & 0.431 & 0.407 & 0.096 & $A 2 g$ & -0.841 & -0.038 & -0.171 & -0.038 \\
\hline
\end{tabular}

\subsection{Blastochloris viridis bacteriochlorophylls}

The dataset comprising the conformations of the $B$. viridis bacteriochlorophyll cofactors was neither as large nor subject to as many experimental variations as that of $R$. sphaeroides described previously. However, we found in our exploratory analysis a single structure that appeared to resolve less cofactor nonplanarity than the rest of the experiments (analogous to RBC2, albeit not as extreme). The principal component analysis ${ }^{*}$ of the data was found to best illustrate the deviation of this structure, namely 1PRC, and the overall consistency of the

* Note that the preference of FA over PCA in the preceding section owed itself to the the rotation of the factors allowing their ease of interpretation; FA could not be used here since this data forms an underdetermined matrix. 
rest. The first PC of the $B$. viridis bacteriochlorophyll cofactors notably correlates with a number of the nonplanarity parameters (Table 3 ) such that the negative score of the outlying structure (1PRC; Figure 7) indicates that its cofactors are consistently less nonplanar than the rest of the data. Note also that this PC accounts for $36.72 \%$ of the total variance in the data and that the scree plot is consistent with there being the only one significant principle component.

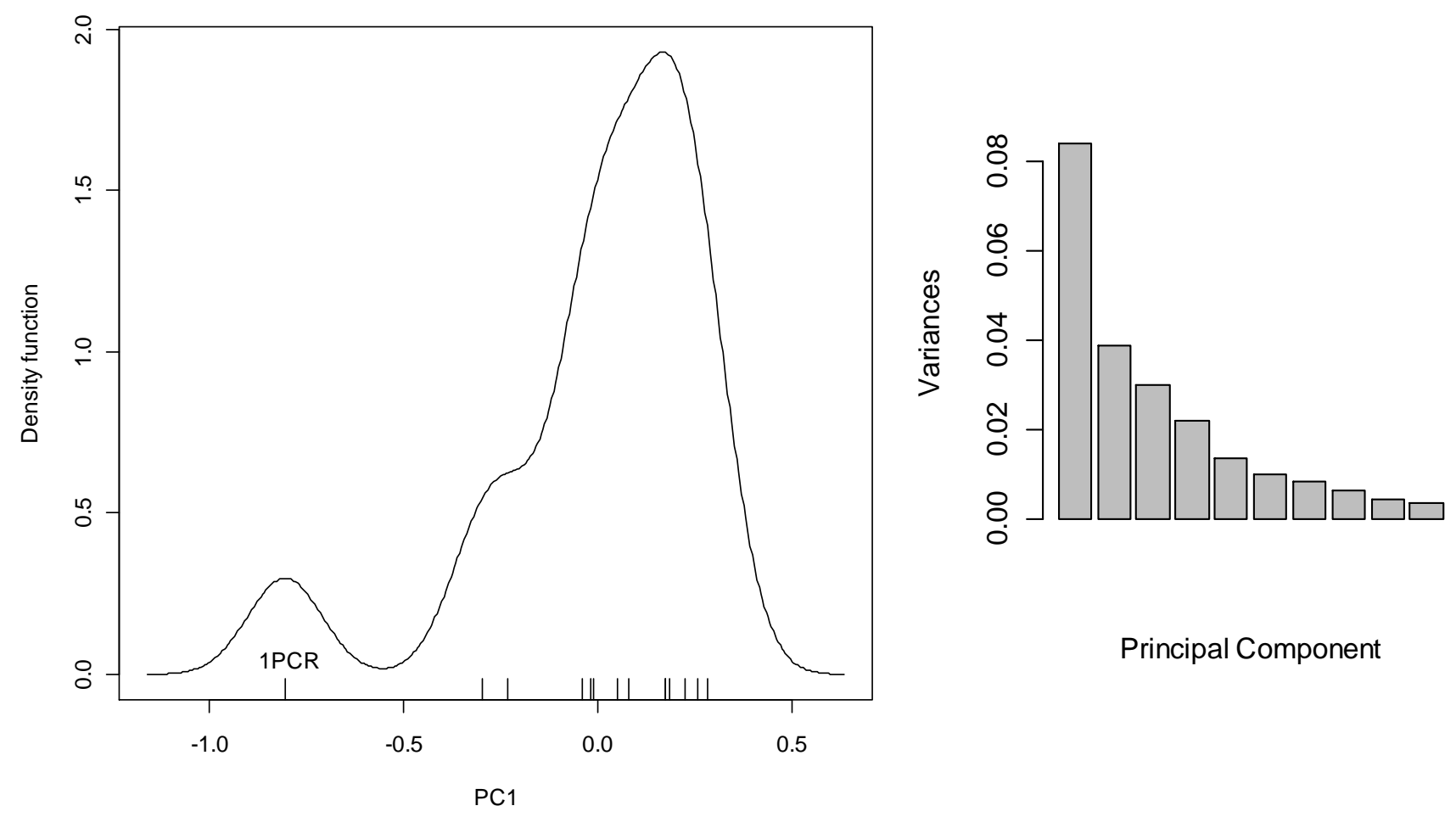

Figure 7: PCA of $B$. viridis bacteriochlorophyll dataset. Kernel density estimate of the distribution of the structure scores on the first



Table 3: Loadings of the original variables onto the first principal component. Loadings in highest $20 \%$ are marked in bold italic.

\begin{tabular}{|c|c|c|c|c|c|c|c|c|c|}
\hline & DM & $\mathrm{DL}$ & BB & $\mathrm{BA}$ & & $\mathrm{DM}$ & $\mathrm{DL}$ & BB & $B A$ \\
\hline Doop & 0.281 & 0.363 & 0.270 & 0.205 & Dip & 0.097 & 0.032 & 0.115 & 0.143 \\
\hline Soop & -0.015 & 0.001 & -0.001 & 0.002 & $\delta i p$ & -0.015 & -0.014 & -0.011 & -0.008 \\
\hline$B 2 u$ & -0.254 & -0.159 & -0.157 & 0.004 & $B 2 g$ & -0.092 & -0.016 & -0.020 & -0.033 \\
\hline$B 1 u$ & -0.278 & -0.344 & -0.048 & 0.039 & $B 1 g$ & -0.147 & 0.019 & -0.137 & -0.168 \\
\hline$A 2 u$ & -0.039 & -0.064 & -0.182 & 0.158 & $E u(x)$ & -0.088 & -0.048 & -0.039 & -0.118 \\
\hline$E g(x)$ & -0.107 & 0.060 & -0.255 & -0.196 & $E u(y)$ & 0.107 & 0.012 & 0.010 & 0.031 \\
\hline$E g(y)$ & 0.059 & -0.046 & -0.005 & 0.003 & $\mathrm{A1g}$ & 0.144 & 0.076 & 0.042 & 0.063 \\
\hline$A 1 u$ & 0.019 & -0.032 & 0.016 & 0.010 & $A 2 g$ & -0.040 & 0.003 & -0.020 & -0.11 \\
\hline
\end{tabular}

\subsection{Blastochloris viridis bacteriopheophytins}

Whilst we found no systematic variation in the $B$. viridis bacteriopheophytin conformations that correlated with the crystal structure determinations during our exploratory analyses, we felt it was appropriate to demonstrate this formally (particularly since this result is in contrast to what was found in the R. sphaeroides set, where experimental correlations in the bacteriochlorophyll data were echoed in their bacteriopheophytins). As confirmation, the PCA of this dataset (Figure 8) did not exhibit any significant structure in either the scree plot (meaning there are no significant PCs) or the distribution of the structure scores (i.e. no readily interpretable features).

However, whilst there is no definite structure to the PCA, the two observations to the right of the plot (Figure 8) flag as potential outliers in the $\mathrm{KDE}$ and were therefore assessed separately; it was found that these structures exhibited in-plane conformations that were quite elongated compared to the rest (Section 5.4.4). 


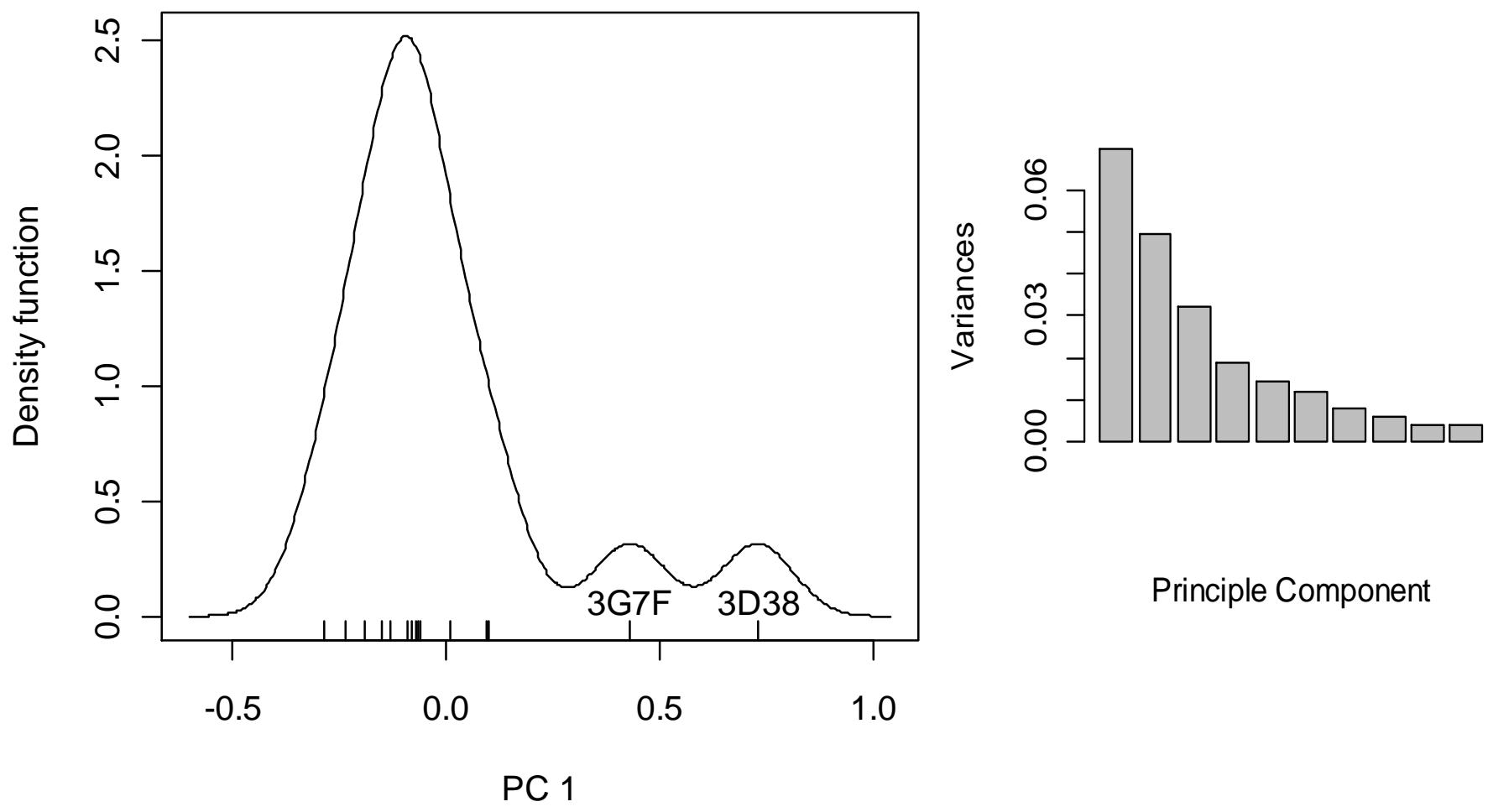

Figure 8: PCA of B. viridis bacteriopheophytin dataset. Kernel density estimate of the distribution of the structure scores on the first principle component (right; bandwidth determined by the plug-in selector, $\mathrm{h}=\mathbf{0 . 0 8 4 1 9 1 3 2}$ ) and PCA scree plot (left).

Table 4: Loadings of the original variables onto the first principal component. Loadings in highest $20 \%$ are marked in bold italic.

\begin{tabular}{|c|c|c|c|c|c|}
\hline & $\Phi A$ & $\Phi B$ & & $\Phi A$ & ФB \\
\hline Doop & 0.017 & 0.020 & Dip & 0.310 & 0.350 \\
\hline Soop & 0.027 & 0.024 & $\delta i p$ & 0.042 & 0.040 \\
\hline$B 2 u$ & 0.083 & 0.296 & $B 2 g$ & 0.111 & 0.223 \\
\hline$B 1 u$ & -0.072 & -0.073 & $B 1 g$ & -0.428 & -0.309 \\
\hline$A 2 u$ & -0.219 & 0.236 & $E u(x)$ & -0.036 & -0.117 \\
\hline$E g(x)$ & 0.114 & -0.063 & $E u(y)$ & -0.042 & 0.002 \\
\hline$E g(y)$ & -0.364 & 0.170 & $A 1 g$ & 0.044 & -0.014 \\
\hline$A 1 u$ & -0.020 & 0.085 & $A 2 g$ & -0.101 & -0.108 \\
\hline
\end{tabular}

\subsection{Assessment of the Quality of the Conformations}

As argued in the justification for the data driven partitioning of the crystal structure determinations (Section 4.1) we believe that the major experimental correlations uncovered in the reaction centre data do not represent any actual differences between cofactor conformations. The need therefore arises for us to assess each of the candidate sets for their potential to deliver reliable structures since it is our view that only one should be correct.

\subsubsection{R. sphaeroides Crystal Structure Sets}

The fact that the experimental types elucidated by each of the $R$. sphaeroides FAs are on the whole correspondent by crystal structure (Table 5, below and Table 41, Section 6), confirms the experimental dependance between the resolved conformations of the BChls and the BPhes. In the case of the $R$. sphaeroides candidates, both the tied structure set (RBC1 and RBP1) as well as the planar structure set (RBC2 and RBP2) of experiments should be rejected and not used for derivation of the mean cofactor geometries. The reasons for this are: the tied structures do not represent independent measurements of the cofactor's geometries and would therefore unduly weight their singular contribution ${ }^{*}$ to an average conformation whereas the planar sets are deemed as erroneous since high-resolution crystal structures of isolated and related molecules (e.g. Mg-chlorins) exhibit significant nonplanarity and also because of the relatively small number of structures exhibiting this

\footnotetext{
* These cofactor structures were most probably obtained via rigid-body refinement based on the cofactor coordinates from the oldest structure in the set; for this reason it would have been acceptable to allow a single structure from RBC1 to contribute to the average conformation but since our final dataset is still quite large it was unnecessary to do so.
} 
feature $(n=18)$. In addition to these arguments, a cursory look at the resolutions of the experiments involved shows that these sets contain structure determinations that are generally on the lower resolution side of the crystal structures (Table 6).

We must then decide between sets RBC3 and RBC4 (and of course, the corresponding bacteriopheophytin sets); sets both exhibiting cofactors that are substantially nonplanar. The key to this decision in our opinion rests in their conformational comparison to related small molecule crystal structures. Recalling that the most obvious feature of the RBC3 crystal structures is that the cofactors all exhibit a striking absence of the A1g distortion (which represents the degree of in-plane macrocycle expansion/contraction) when compared to each of the other subsets; we have found in our investigations of isolated chlorin crystal structures that this deformation is, without exception, almost entirely dependent on the central ion that is present in the macrocycle. Thus, the A1g deformation exhibited by the cofactors in RBC3 is in fact that of a metalated Cu macrocycle whilst that exhibited by RBC4 (and indeed the other subsets as well as the $B$. viridis bacteriochlorophylls) is consistent with $\mathrm{Mg}$ coordinated in the central cavity (specifically, we have compared them to Mg-chlorophyllides). In addition, the excessive higher energy distortions exhibited by the RBC3 cofactors (this feature is also shared with RBP3) are quite unprecedented in porphyrinoid cofactor conformations that are induced by protein interaction alone, since the energies required for their induction are significantly greater than that required by a combination of only lowest-energy normal-deformations. Finally, if the previous arguments are not sufficient, then it should be noted that the vast majority of the cofactors in this set (RBC3) were part of the same study (see Section 6.1); most of them were made at the same time by the same group and all of them were diffracted in the same place. Thus we suppose that at some point during their measurement or refinement a systematic error has been introduced (which we cannot specifically identify at this time) that has gone unnoticed since the goal of their study was quite different from determination and analysis of accurate conformations of the bacteriochlorophyll cofactors. It is for these reasons, and the appearance of the set's independence of measurement, that we believe the RBC4 $(n=32)$ structures to be the best candidates to provide accurate and reliable cofactor conformations.

Table 5: Contingency table of PDB structure count in RBC group (columns) and RBP group (rows). OL = outlier; NA = not included in factor analysis and 4, 5 (RBP group) = structures containing two RCs in asymmetric unit with one classed as RBP4 and the other as RBP5.

\begin{tabular}{l|llllll} 
& 1 & 2 & 3 & 4 & OL & NA \\
\hline 1 & 9 & & & 1 & & \\
2 & & 13 & & & & 1 \\
3 & & & 14 & & 3 & \\
4 & & & & 13 & & \\
OL & & & 1 & 5 & 2 & 1 \\
NA & & 2 & 1 & 3 & & \\
5 & & & & 2 & 2 & 1 \\
4,5 & & & & 3 & &
\end{tabular}

Table 6: Summary of crystal structures present in each set. [a] mean in parenthesis, [b] relates to structures with two RCs in asymmetric unit where each RC was classified differently as RBP4 or RBP5.

\begin{tabular}{|c|c|c|}
\hline & Crystal structures & Resolution(s) ${ }^{[a \mid}$ \\
\hline Total in PDB & 77 & $1.80-4.60(2.66)$ \\
\hline \multicolumn{3}{|c|}{ R. sphaeroides bacteriochlorophyll factor analysis } \\
\hline RBC1 & 9 & $2.70-3.20(3.02)$ \\
\hline $\mathrm{RBC2}$ & 15 (18 RCs) & $2.10-4.60(2.95)$ \\
\hline $\mathrm{RBC3}$ & 16 & $1.87-2.88(2.36)$ \\
\hline RBC4 & 27 (32 RCs) & $1.80-3.00(2.53)$ \\
\hline $\mathrm{OL}$ & 7 & $2.20-3.10(2.76)$ \\
\hline NA & 3 & $2.50-3.00(2.77)$ \\
\hline \multicolumn{3}{|c|}{ R. sphaeroides bacteriopheophytin factor analysis } \\
\hline RBP1 & 10 & $2.10-3.20(2.93)$ \\
\hline RBP2 & 14 (16 RCs) & $2.35-4.60(2.99)$ \\
\hline RBP3 & 17 & $1.87-2.88(2.36)$ \\
\hline RBP4 & $13(+3,16 \mathrm{RCs})^{[\mathrm{b}]}$ & $2.30-3.00(2.61)$ \\
\hline RBP5 & $5(+3,10 R C s)^{[b]}$ & $2.20-3.10(2.68)$ \\
\hline $\mathrm{OL}$ & 9 & $2.55-2.90(2.70)$ \\
\hline NA & 6 & $1.80-2.80(2.32)$ \\
\hline
\end{tabular}




\subsubsection{B. viridis Crystal Structure Sets}

For the $B$. viridis bacteriochlorophyll cofactors, the arguments stated above for the exclusion of the planar cofactor set, apply also to our decision in proceeding with their analysis using the BBC2 set of structures and not with structure 1PRC, even though the bacteriopheophytin cofactors of 1PRC do not reflect this feature. This lack of corroboration can be explained though since it is possible that different restraints have been applied to the bacteriopheophytins in this structure and so, since the conformations of the BChl cofactors of 1 PRC are all consistently and significantly less distorted than the rest, we feel it to remain reasonable to assume that this aspect is an experimental artefact.

As for the apparent outliers in the B. viridis bacteriopheophytin PCA, assessment of their individual conformations showed that the reason for this was that on the whole, the structures exhibited greater in-plane distortion as well as changes to the out-of-plane distortion pattern (Section 5.4.4).

\section{Macrocycle Conformations}

\subsection{Illustrations of the Lowest Energy Normal-deformations}



$\mathrm{E}_{\mathrm{g}(\mathrm{x})} / \operatorname{wave}(x)$

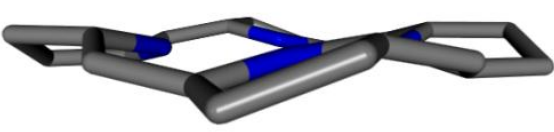

$\mathrm{B}_{1 \mathrm{u}} /$ ruffle

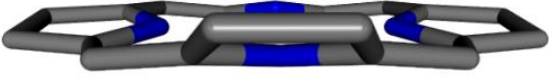

$\mathrm{E}_{\mathrm{g}(\mathrm{y})} / \operatorname{wave}(y)$

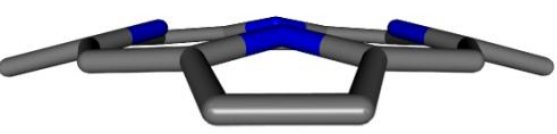

$\mathrm{A}_{2 \mathrm{u}} /$ dome



$\mathrm{A}_{1 \mathrm{u}} /$ propeller

Figure 9: Illustrations of the six lowest-energy out-of-plane normal-deformations.

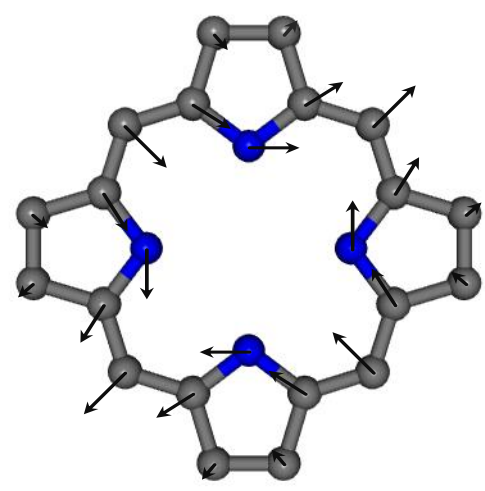

$\mathrm{B}_{2 \mathrm{~g}} /$ meso-stretch

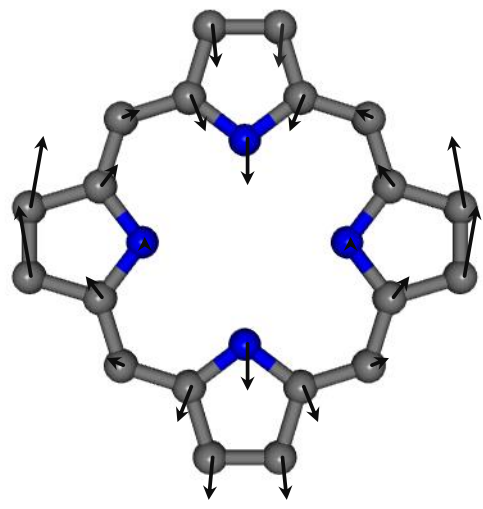

$\mathrm{E}_{\mathrm{u}(\mathrm{y})} /$ pyr-translate(y)


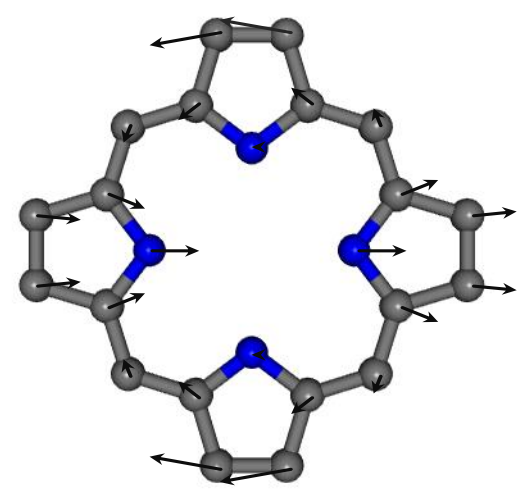

$\mathrm{E}_{\mathrm{u}(\mathrm{x})} /$ pyr-translate $(x)$

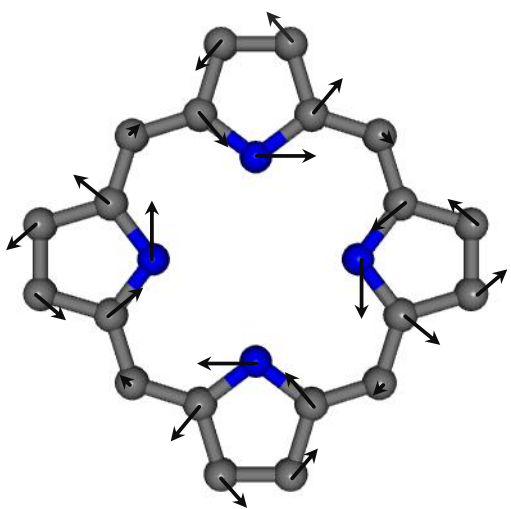

$\mathrm{A}_{2 \mathrm{~g}} /$ pyr-rotate

Figure 10: Illustrations of the six lowest-energy in-plane normal-deformations. 


\subsection{Nomenclature of Cofactor Nuclei and Ring Subunits}

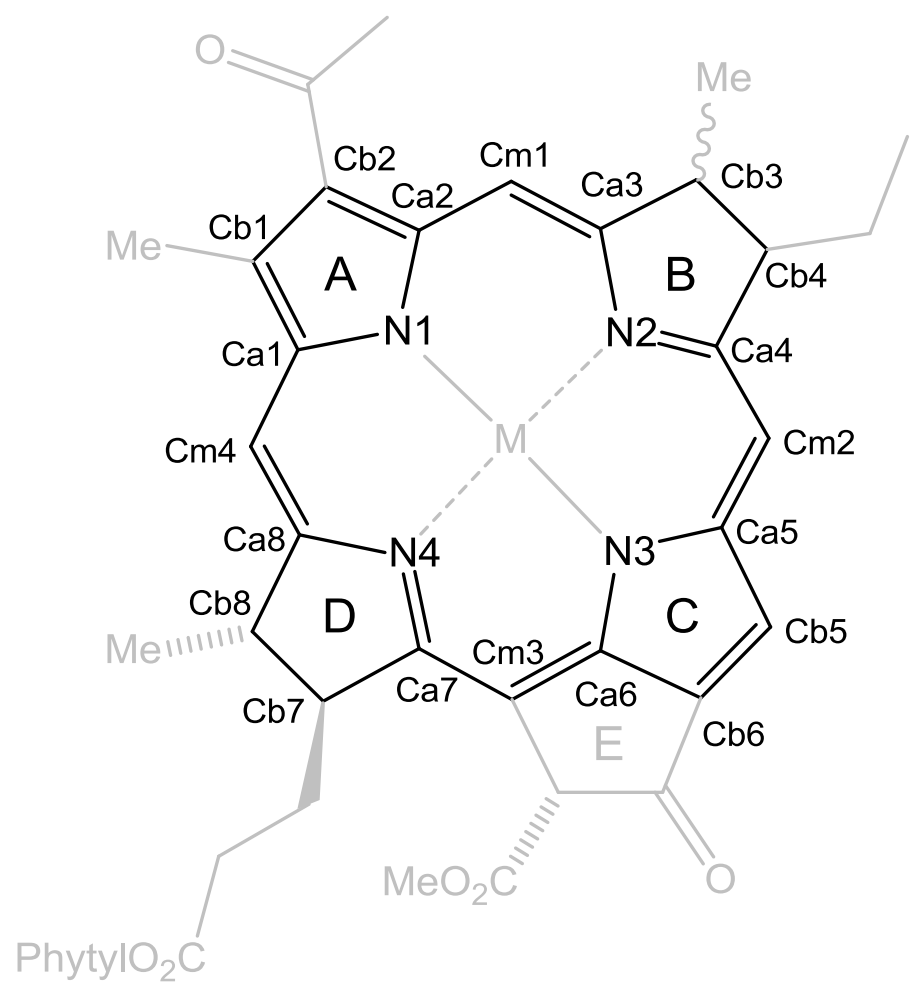

Figure 11: Nomenclature of cofactor nuclei and ring subunits used throughout.

\subsection{Overviews of Most Relevant Cofactor Conformations}

\subsubsection{R. sphaeroides $\mathrm{RBC} 4$ cofactors}

The minimum basis was deemed to be acceptable since the mean deviation between the simulated and actual conformations, $\delta$ oop values (Section 5.6.3 for descriptives of RBC4 dataset and Section 5.7.1 for Doop of mean conformation), were either within or close to Jentzen et al's criterion $^{[2 \mathrm{~b}]}$ (i.e. close to 0.03 ). From the NSDs (Section 5.4.1), it is immediately apparent that the cofactors assume statistically significant, unique nonplanar geometries and share a much more common IP conformation, showing that the IP conformation is mostly effected by the chemical structure and that the OOP deformation is controlled by the protein/cofactor environment. The key features of the NSDs of the cofactors are that BA is the most planar; BB is the most nonplanar, exhibiting a large B1u distortion; DL is quite nonplanar and exhibits a dominant $\mathrm{A} 2 \mathrm{u}$ distortion with contributions from $\mathrm{B} 2 \mathrm{u}$ and $\mathrm{B} 1 \mathrm{u}$, and $\mathrm{DM}$ is considerably nonplanar showing significant $\mathrm{B} 2 \mathrm{u}$ distortion and a large, negative B1u deformation (see Doop values in Section 5.6.3 and 5.7.1). In terms of skeletal displacements (Section 0), DL stands apart in that rings $A, B$ and $C$ (for nomenclature see Section 5.2) exhibit a 'tilt' in one direction along their $\mathrm{C} \alpha-\mathrm{C} \alpha$ axes, which originates from the A2u mode, although the OOP orientation of ring $A$ may be somewhat conserved among the four. It should also be readily observed that the only real failing of the minimum basis is that the $S P^{3}$-hybridisation of the $\beta$-carbons of the reduced rings $(B$ and $D$ ) that leads to their displacement above and below the plane is not entirely encapsulated (red/blue comparison, Section 0 ). This validates the use of the minimum basis for the conformational comparisons of these structures in our paper since these must be the distortions that are induced by the protein/cofactorand, in the case of the special-pair, the cofactor/cofactor interactions.

In addition, it is also noteworthy to draw attention to the differences of the Doop and סoop parameters between the descriptive statistics of the RBC4 dataset and their re-centred NSDs (i.e. the values of these parameters for the mean conformation). In particular, the reduction in the

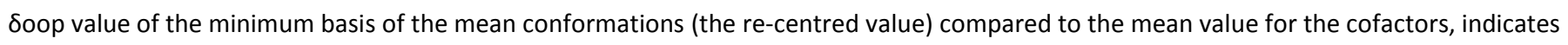
that the minimum basis provides a better description of the mean conformation than it does for most of the individual observations. It is possible to interpret this fact as showing that the averaging process has resulted in the removal of spurious higher-energy distortions. Considering this together with the knowledge that the remaining higher-energy distortions are required only to describe the $\mathrm{SP}^{3}$-hybridisation of the $\beta$-carbons of the reduced rings (as noted above) we may conclude that the force exerted by the protein upon the cofactor is only energetically capable of inducing these lower energy distortions.

\subsubsection{B. viridis $\mathrm{BBC1}$ cofactors}

Here it was found that the extended basis was required to obtain sufficient agreement between the simulated conformations and the observed mean conformation (to ensure that $\delta_{\text {oop }}$ was close to 0.03 ; Section 5.4.2) mostly due to, as the next-to-lowest energy deformations

\footnotetext{
* Of course, this conclusion is dependent upon our being correct about the erroneous nature of the RBC3 structures.
} 
show (Figure 18, Section 5.4.2), a strong $B 1 \mathrm{u}(2)^{*}$ and $\mathrm{A} 1 \mathrm{u}(2)$ distortion in each of the special-pair cofactors and also a strong $\mathrm{Eg}(\mathrm{x})(2)$ deformation in the accessory bacteriochlorophyll monomers (in particular of BB). In contrast to $R$. sphaeroides, the A/B-branch asymmetry of the special-pair originates from the extent rather than the make-up of the conformation (thus DL exhibits a large negative B1u distortion, although to a lesser extent than DM and does not show the A2u mode that contributed to DLs uniqueness in R. sphaeroides; ) and the accessory bacteriochlorophyll monomers do not exhibit A/B-branch asymmetry of the order that was found in $R$. sphaeroides (although note the considerably larger $\operatorname{Eg}(x)(2)$ distortion of BB). Similar to the case for $R$. sphaeroides and the adequacy of the minimum basis in that case, here the extended basis captures the majority of the cofactors' conformations (Section 5.5.2) excepting the localised perturbation of the $\mathrm{SP}^{3}$ hybridisation of the $\beta$-carbons on rings $B$ and $D$.

\subsection{Magnitude of the Physicochemical Effects of the Cofactor Distortions}

One way to assess the physicochemical impact of the observed macrocyclic distortion in the RC in the context of their relative importance compared to the other forms of functional modulation that the protein scaffold is known to exert, it is necessary to approximate the distortions' perturbation energy (deformation energy) and to then compare these energies with those that have been determined for the other factors. These deformation energies can be obtained directly from the NSD analysis ${ }^{[2 \mathrm{~b}]}$ since it has been shown that the total macrocycle distortion energy $\left(E_{d}\right)$ is obtained from the linear sum of the contributions of each of the normal-deformations $\left(\delta E_{i}\right)$, in turn given by the square of the normal-coordinate displacement $\left(d_{i}^{2}\right)$ multiplied by the respective force constant of the mode $\left(K_{i}\right)$, as shown below:-

$$
E_{d}=\sum_{i=1}^{n} \delta E_{i}=\sum_{i=1}^{n} K_{i} d_{i}^{2}
$$

The energies then give an idea of the potential magnitude of the effect that can be induced by macrocyclic distortion on an energetically determined quantity (e.g., reduction potential or absorption maximum). The force constants used were calculated by Jentzen et $a l^{[2 \mathrm{~b}]}$ and are expected to give reasonable estimates of the distortion energies for this purpose.

Table 7: Total macrocycle distortion energies derived from minimum basis NSD of each cofactors mean conformation.

\begin{tabular}{|l|l|l|l|l|l|l|l|l|}
\hline & \multicolumn{4}{l|}{ R. sphaeroides } & \multicolumn{3}{l|}{ B. viridis } \\
\hline & $\mathrm{BA}$ & $\mathrm{BB}$ & $\mathrm{DL}$ & $\mathrm{DM}$ & $\mathrm{BA}$ & $\mathrm{BB}$ & $\mathrm{DL}$ & $\mathrm{DM}$ \\
\hline$E_{d} / \mathrm{kJ} \mathrm{mol}^{-1}$ & 5.8941 & 10.2961 & 8.2253 & 6.2451 & 11.4459 & 10.1356 & 16.4346 & 30.5355 \\
\hline$E_{d} / \mathrm{meV}_{\text {molecule }}^{-1}$ & 61 & 107 & 85 & 65 & 119 & 105 & 170 & 317 \\
\hline
\end{tabular}

Table 8: Influences of both H-bonding to- and the introduction of polarised residues near P870 (data from Allen and Williams ${ }^{[5]}$ ).

\begin{tabular}{|l|l|l|}
\hline & H-bonding & Polarised residues \\
\hline Qy-band & $\begin{array}{l}-8 \text { to }-22 \mathrm{~nm} \text { shift (blue-shift) of Qy upon } \\
\text { removal of DL acetyl-HIS H-bond }\end{array}$ & Unavailable \\
\hline $\begin{array}{l}\mathrm{P} / \mathrm{P}^{\bullet+} \text { Midpoint } \\
\text { potential }\end{array}$ & $\begin{array}{l}420 \text { to } 765 \mathrm{mV}(+60-125 \mathrm{mV} / \mathrm{H} \text {-bond; 100meV } \\
\text { Hückel model stabilisation) }\end{array}$ & $\begin{array}{l}(\Delta)-60 \text { to }+50 \mathrm{mV} \text { (-ve charge / +ve charge 10 } \mathrm{A} \text { from donor; exponential } \\
\text { dependence on distance and dielectric constant) }\end{array}$ \\
\hline
\end{tabular}

Comparison of the estimated conformational distortion energies and experimental values of physicochemical changes brought about by other protein interactions derived from RC mutagenesis studies ${ }^{[5]}$ (Table 7Table 8) reveals that the nonplanar distortions represent energetic perturbations on a par with those brought about by $\mathrm{H}$-bonding and electrostatic influences. For example, the estimated $100 \mathrm{meV}$ stabilisation induced by each additional $\mathrm{H}$-bond to an acetyl-oxygen of a $\mathrm{BChl}$ constituent of the special-pair is certainly matched by the range of distortion energies exhibited by the cofactors (Table 7). This approach is valid on the basis of simple MO theory considering that the distortion of the macrocycle from its preferred geometry raises the energy of the MOs, including the HOMO, which affects decreased ionisation energy and consequently a decreased midpoint potential. Furthermore, this facile analysis may be improved somewhat by an assumption, employed by Zucchelli et $a l,{ }^{[6]}$ that a distortion of a given symmetry modifies the energy-level of an orbital with the same symmetry; this then allows specific estimation of the effect of the cofactor conformations on the Gouterman frontier orbitals ${ }^{[7]}$ of the BChls enabling a more balanced approach and a direct assessment of the conformationally induced spectral shift of the Qy-band.

Briefly, the four-orbital model describes the electronic structure of porphyrins by a configuration interaction between the HOMO, HOMO-1, LUMO and LUMO-1 of the conjugated $\pi$-system which gives rise to the high energy Soret and low energy Q-bands observed in their UV/Vis absorption spectra. ${ }^{[7]}$ The symmetries of these orbitals, when the model is applied to the D2h symmetric BChl macrocycle are Au, B1u, B2g and $\mathrm{B} 3 \mathrm{~g}$, respectively. ${ }^{[8]}$ Furthermore, after considering how the NSD basis-modes transform in the D2h point-group, the normal-modes corresponding to these symmetries are the $\operatorname{ruf}, \operatorname{dom}, \operatorname{wav}(x)$ and $\operatorname{wav}(y)$ distortions (details not shown). The individual contributions to the

\footnotetext{
"The parenthetic " 2 " following the symmetry of the distortion denotes that it is the second lowest, or "next-to-lowest", energy distortion of that symmetry that is referred to.
} 
total deformation energies $\left(\delta E_{i}\right)$ for each of the NSD minimum basis modes of these symmetries for each cofactor are presented (Table 9 ) as well as this models consequent effect on the four-orbital transitions (Table 10).

Table 9: Normal-deformation energies of the modes of the minimum basis associated with the two highest and lowest energy molecular orbitals of the BChl cofactors by symmetry.

\begin{tabular}{|c|c|c|c|c|c|c|c|c|c|c|}
\hline \multirow[t]{3}{*}{ Orbital } & \multirow[t]{3}{*}{ D2h orbital symmetry ${ }^{[8]}$} & \multirow[t]{3}{*}{ Matching (D2h) normal-mode(s) } & \multicolumn{8}{|c|}{ Perturbation / meV } \\
\hline & & & \multicolumn{4}{|c|}{ R. sphaeroides } & \multicolumn{4}{|c|}{ B. viridis } \\
\hline & & & BA & BB & $\mathrm{DL}$ & DM & $\mathrm{BA}$ & BB & $\mathrm{DL}$ & DM \\
\hline LUMO+1 & B3g & saddle / wave (y) [reduced axis] & 0 & 16 & 24 & 7 & 5 & 3 & 8 & 16 \\
\hline LUMO & B2g & wave $(\mathrm{x})$ & 51 & 53 & 6 & 7 & 98 & 90 & 0 & 3 \\
\hline HOMO & $\mathrm{Au}$ & ruffle & 1 & 31 & 6 & 35 & 3 & 0 & 79 & 157 \\
\hline HOMO-1 & B1u & dome & 9 & 2 & 48 & 4 & 1 & 12 & 1 & 1 \\
\hline
\end{tabular}

Table 10: Distortion induced shift of the LUMO $\leftarrow$ HOMO and LUMO+1 $\leftarrow$ HOMO-1 transitions predicted by like-symmetry assumptions of Zucchelli et $a l^{[6]}$.

\begin{tabular}{|c|c|c|c|c|c|c|c|c|}
\hline & \multicolumn{4}{|c|}{ R. sphaeroides } & \multicolumn{4}{|l|}{ B. viridis } \\
\hline & $\mathrm{BA}$ & $\mathrm{BB}$ & $\mathrm{DL}$ & $\mathrm{DM}$ & $\mathrm{BA}$ & $\mathrm{BB}$ & $\mathrm{DL}$ & $\mathrm{DM}$ \\
\hline$\Delta \mathrm{E}_{\mathrm{B} 2 \mathrm{~g} \leftarrow \mathrm{Au}} / \mathrm{meV}$ & 50 & 22 & 0 & -28 & 95 & 90 & -79 & -154 \\
\hline$\Delta \mathrm{E}_{\mathrm{B} 3 \mathrm{~g} \leftarrow \mathrm{B} 1 \mathrm{u}} / \mathrm{meV}$ & -9 & 14 & -24 & 3 & 4 & -9 & 7 & 15 \\
\hline
\end{tabular}

Since it has been shown that the $\mathrm{B} 2 \mathrm{~g} \leftarrow$ Au transition contributes greater than $90 \%$ of the Qy-band transition, ${ }^{[8]}$ the first row of entries in Table 10 provides a good estimate of the spectral shift due to conformational distortion (relative to a planar macrocycle). Thus, a blue-shift is predicted for all of the $\mathrm{BChl}$ accessory monomers, a slight red-shit for DM of $R$. sphaeroides and a considerable red-shift of the components of the B. viridis special-pair (P960). Thus, taking the solution Qy-absorption maximum of $795 \mathrm{~nm}$ for BChl $\mathrm{b}$ as reference ${ }^{[9]}$ (in this model, assumed to be planar), $\triangle E_{B 2 \mathrm{~g} \leftarrow A u}$ for $D L$ predicts a shift to $837 \mathrm{~nm}$ whilst in $D M$ the estimated maximum would be shifted to $882 \mathrm{~nm}$. Within the limits of the preceding approximations then, the red-shift of P960 relative to P870 may be assisted as much by conformational control as it is by the greater excitonic coupling of the former special-pair.

Another approach to the question of the magnitude of the physicochemical changes associated with the varying degrees of distortion of the cofactors is to draw on experimental results from conformationally designed synthetic porphyrins. The relevant results from two such studies concerning the effect of a gradual sterically induced, ruffle distortion of nickel porphyrins on redox and absorption properties, ${ }^{[10]}$ and that of a graded saddle distortion of free-base and zinc porphyrins on their photophysical behaviours ${ }^{[11]}$ have been extracted and are illustrated below (Figure 12 Figure 13). These studies also demonstrate that the potential physicochemical effects of macrocycle distortion of the extent observed in the RC are significant in the context of the other protein modulated influences.


Figure 12: Illustration of the relation between the total out-of-plane distortion (Doop) and the energy of the Qy-band (left) and the first half-wave oxidation potential (right) a a series of conformationally designed, sterically crowded ruffle Ni-porphyrins; data from Senge et $a l^{[10]}$.

For example, the approximate one angstrom shift in the total distortion of $(5,10,15,20$-tetrabutyl-2,3,7,8,12,13,17,18octaethylporphyrinato)nickel(II) (NiTBUOEP) relative to $(2,3,7,8,12,13,17,18$-octaethylporphyrinato)nickel(II) (NiOEP) leads to a $0.3 \mathrm{~V}$ reduction in the first half-wave oxidation potential a $0.29 \mathrm{eV}$ reduction in the Qy-band of the former relative to the latter ${ }^{[10]}$ (Figure 12); this level of 
distortion is similar to that exhibited by the special-pair cofactors of $B$. viridis. Concerning redox properties again, in a recent study by Olea et $a l^{[12]}$ mutations to the haem binding pocket of H-NOX, decreasing the total degree of distortion from $2.180 \AA$ in the wild-type to $0.870 \AA$ in the mutant affected a decrease of the haem midpoint potential by $171 \mathrm{mV}$. Note that this reversal in the trend between macrocycle distortion and redox potential is of course due the fact that the haem oxidation is a metal centred process. The relevance of this result to the $\mathrm{BChl}$ redox processes (macrocycle centred) is only apparent when one considers that reduction potentials are related to electron density; applying a pseudo-conservation principle coupled with the knowledge that the distortion of the wild-type H-NOX cofactor was confirmed to affect a measured decrease in electron density at the iron-centre, ${ }^{[12]}$ renders the proposal acceptable that potentials of macrocycle and metal-centred redox process, when affected predominantly by conformational effects, are inversely proportional. As another example, the decreasing singlet oxygen yields affected by a gradually increasing saddle distortion ${ }^{[11]}$ (Figure 13) as a consequence of the distortions' effects on numerous photo-physical parameters ${ }^{[11]}$ lends weight to the idea of a photo-protective role of the distortion of the BB cofactor in R. sphaeroides.
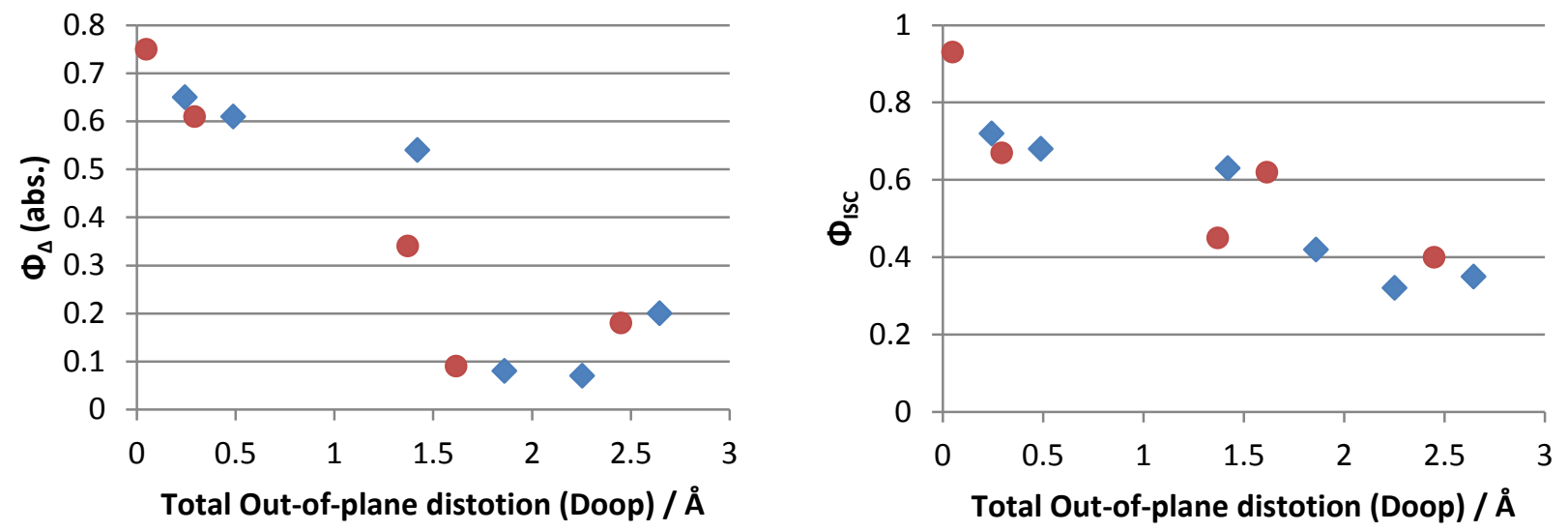

Figure 13: Illustrations of the association between the total out-of-plane distortion (Doop) and the singlet-oxygen (left) and the intersystem crossing quantum yields of a series of conformationally designed free-base- (blue diamonds) and $\mathrm{Zn}$ (red circles) saddled porphyrins; data from Roder et al $^{[11]}$.

\subsection{NSD Descriptive Statistics}

\subsubsection{Rhodobacter sphaeroides bacteriochlorophylls}
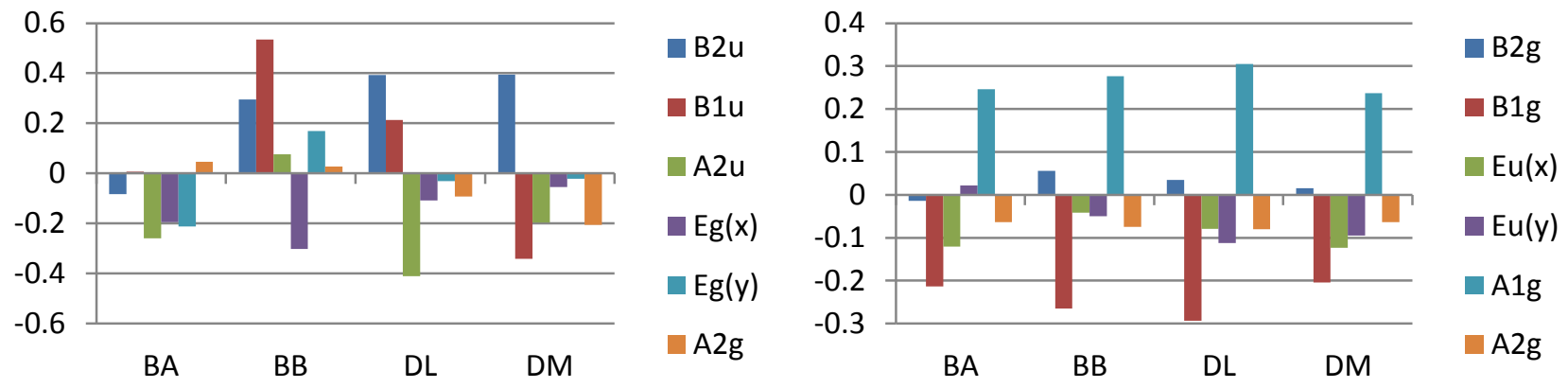

Figure 14: Mean minimum basis normal-coordinate displacements of each ETC cofactor in RBC1 ('no error set'). Out-of-plane (left) and inplane (right). Y-axes in angstroms; error bars indicate two standard errors (not visible since all are less than $0.00023 \AA ̊$ ). $n=9$.
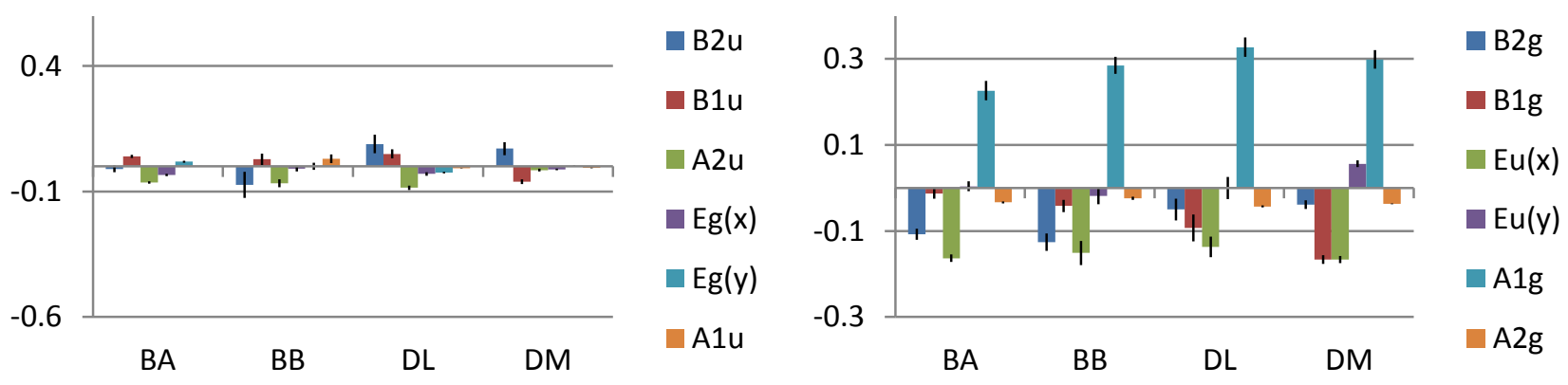

Figure 15: Mean minimum basis normal-coordinate displacements of each ETC cofactor in RBC2a ('planar set'). Out-of-plane (left) and inplane (right). $Y$-axes in angstroms; error bars indicate two standard errors. $n=8$. 



Figure 16: Mean minimum basis normal-coordinate displacements of each ETC cofactor in RBC2b ('planar set'). Out-of-plane (left) and inplane (right). $Y$-axes in angstroms; error bars indicate two standard errors. $n=10$.
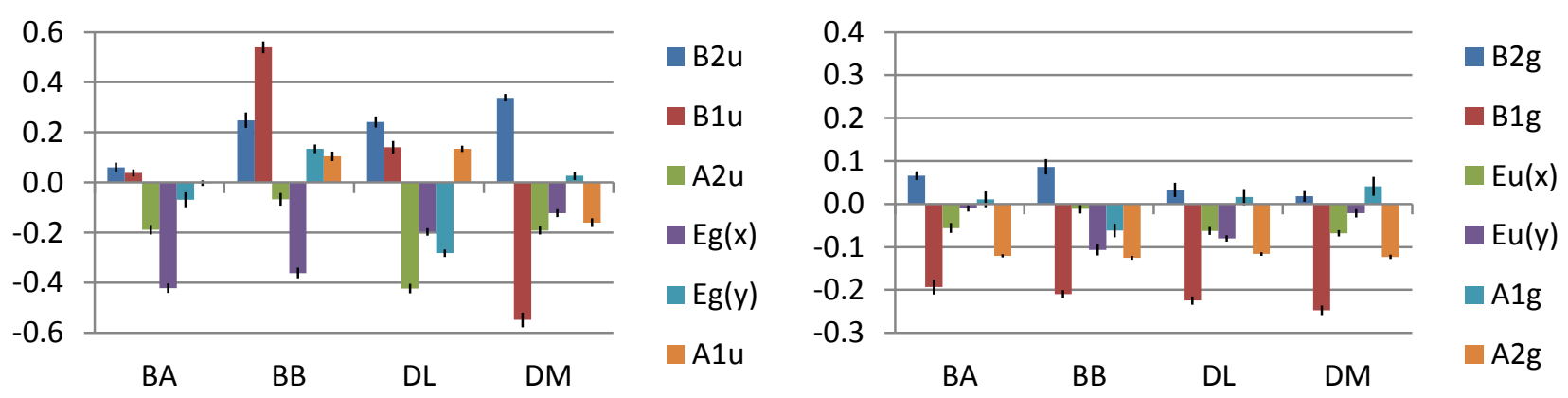

Figure 17: Mean minimum basis normal-coordinate displacements of each ETC cofactor in RBC3 ('unusual IP conformation set'). Out-ofplane (left) and in-plane (right). Y-axes in angstroms; error bars indicate two standard errors. $\mathrm{n}=16$.
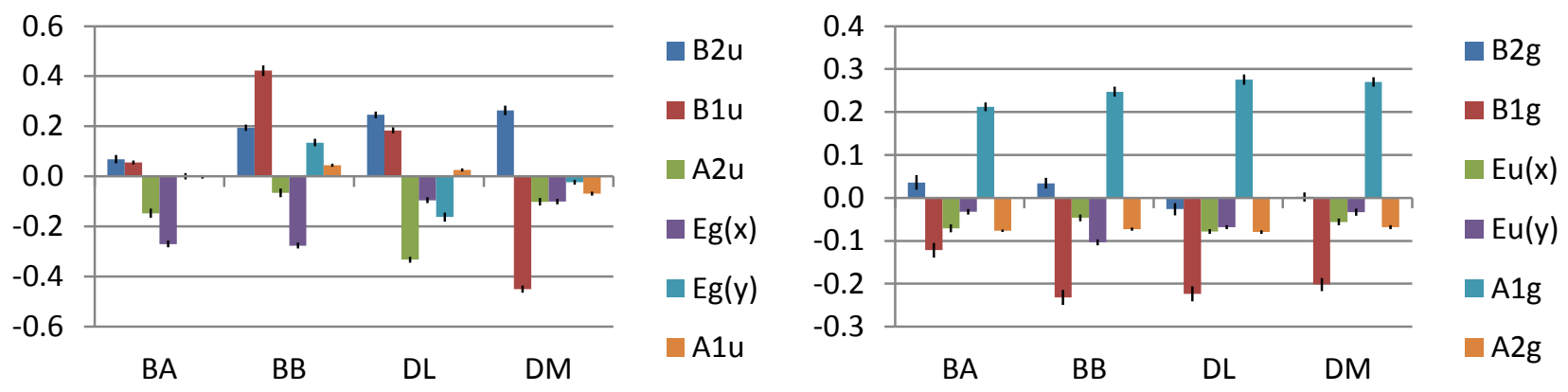

Figure 18: Mean minimum basis normal-coordinate displacements of each ETC cofactor in RBC4 ('deemed as most reliable set'). Out-ofplane (left) and in-plane (right). $Y$-axes in angstroms; error bars indicate two standard errors. $\mathbf{n}=32$. 
5.5.2 Blastochloris viridis bacteriochlorophylls
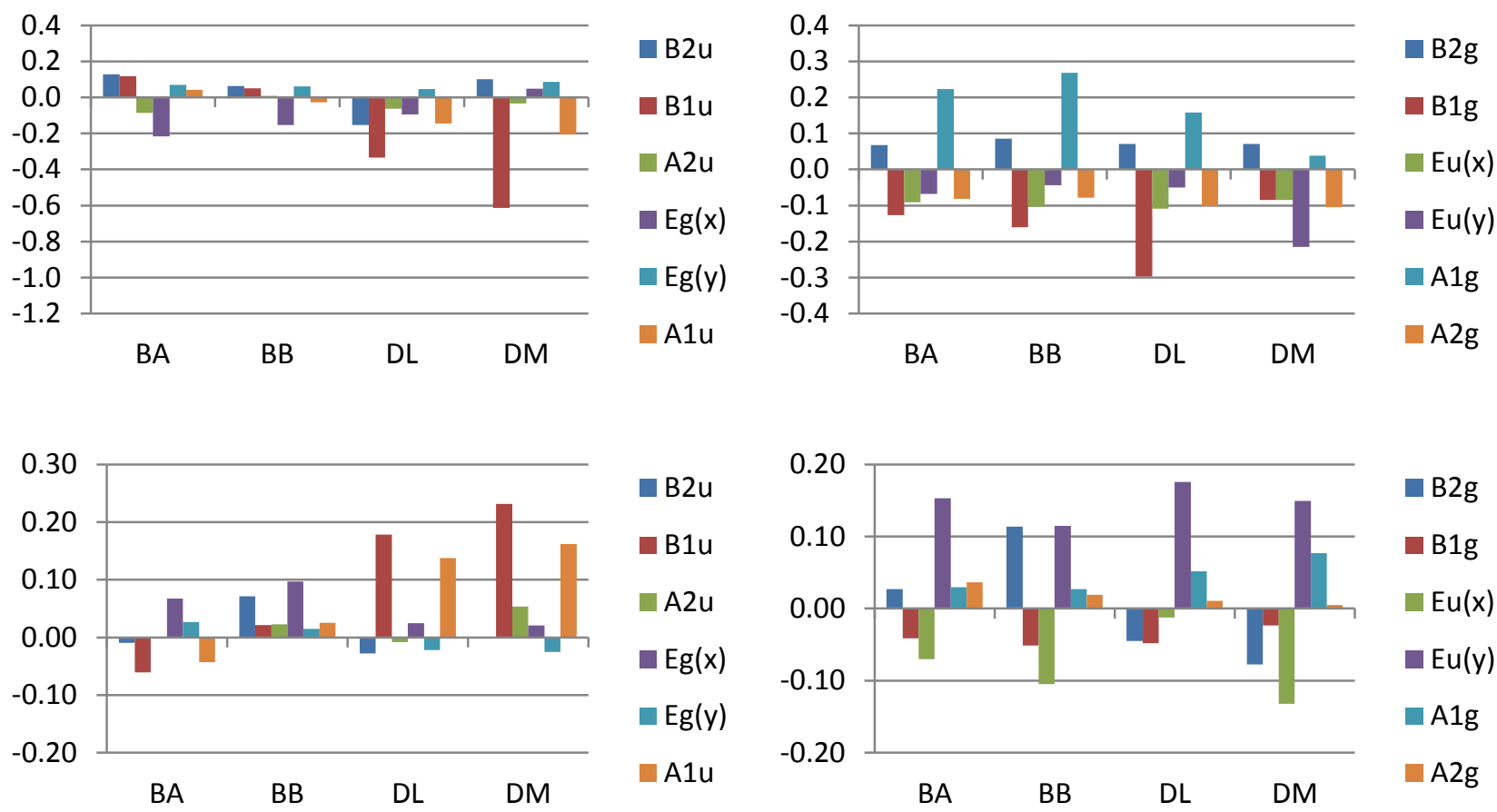

Figure 19: Extended basis normal-coordinate displacements of each ETC cofactor in 1PRC. Out-of-plane (left), in-plane (right), lowest energy (top) and next-to-lowest energy (bottom). Y-axes in angstroms.
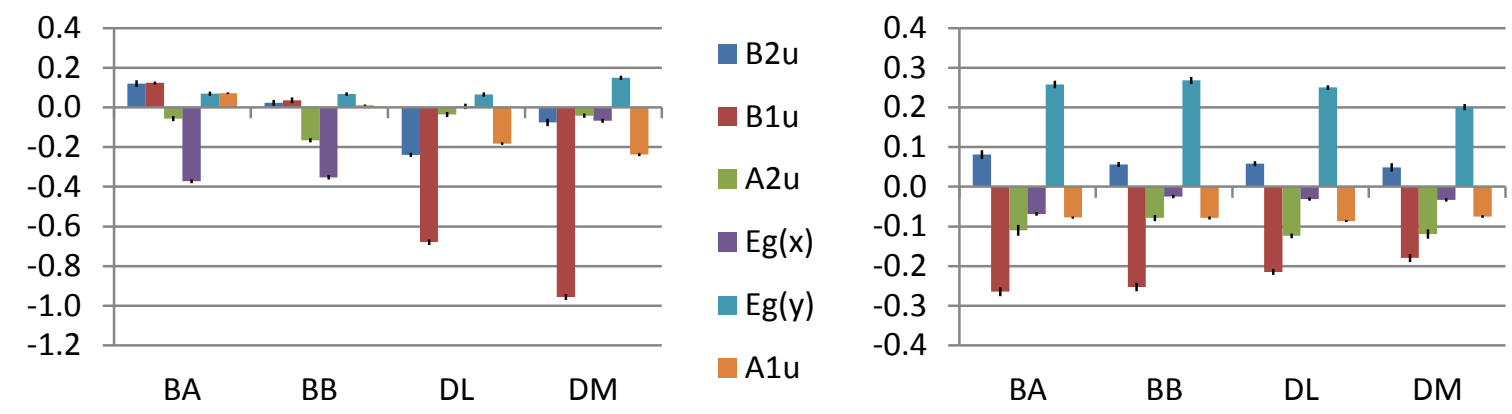

$$
\begin{aligned}
& \text { B2g } \\
& \text { B1g } \\
& \text { Eu(x) } \\
& \text { Eu(y) } \\
& \text { A1g } \\
& \text { A2g }
\end{aligned}
$$
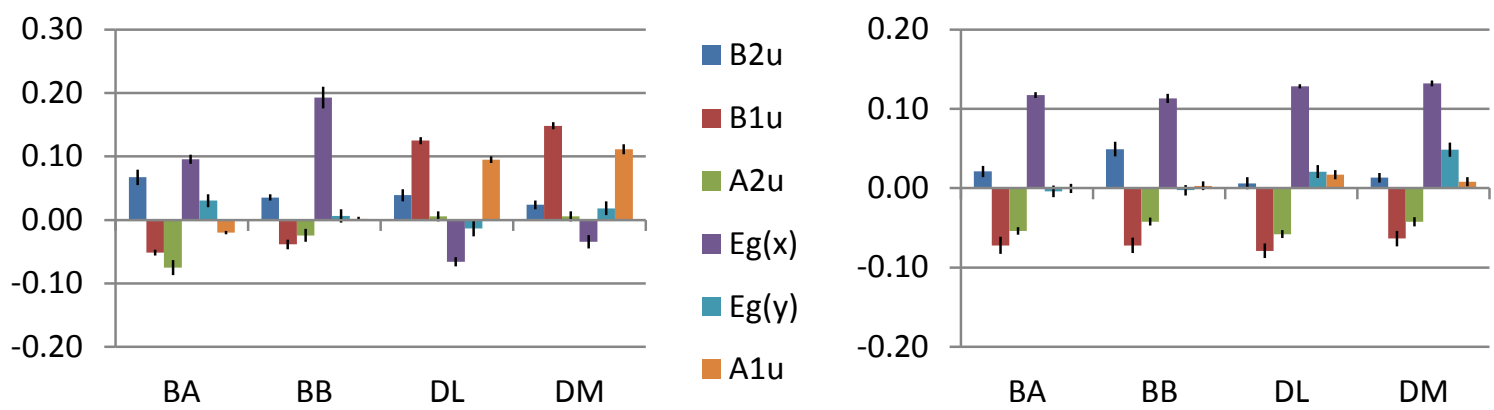

$$
\begin{aligned}
& B 2 g \\
& B 1 g \\
& E u(x) \\
& E u(y) \\
& A 1 g \\
& A 2 g
\end{aligned}
$$

Figure 20: Mean extended basis normal-coordinate displacements of each ETC cofactor in BBC1. Out-of-plane (left), in-plane (right), lowest energy (top) and next-to-lowest energy (bottom). Y-axes in angstroms; error bars indicate two standard errors; $\mathbf{n}=13$. 
5.5.3 Rhodobacter sphaeroides bacteriopheophytins


Figure 21: Mean minimum basis normal-coordinate displacements of each ETC cofactor in RBP2. Out-of-plane (left) and in-plane (right). Yaxes in angstroms; error bars indicate two standard errors and $n=16$.
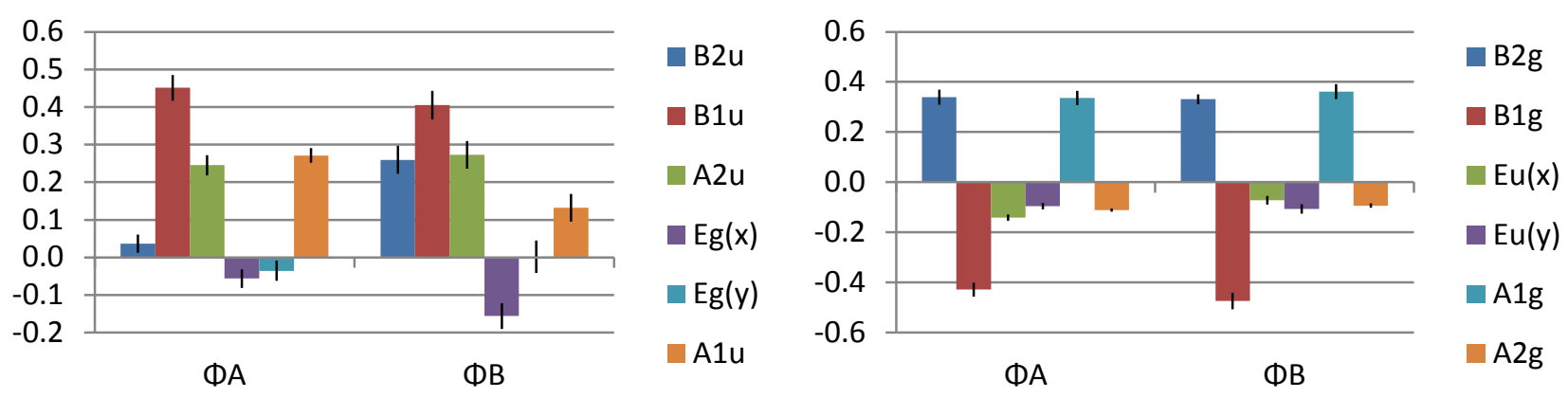

Figure 22: Mean minimum basis normal-coordinate displacements of each ETC cofactor in RBP3. Out-of-plane (left) and in-plane (right). Yaxes in angstroms; error bars indicate two standard errors and $n=17$.
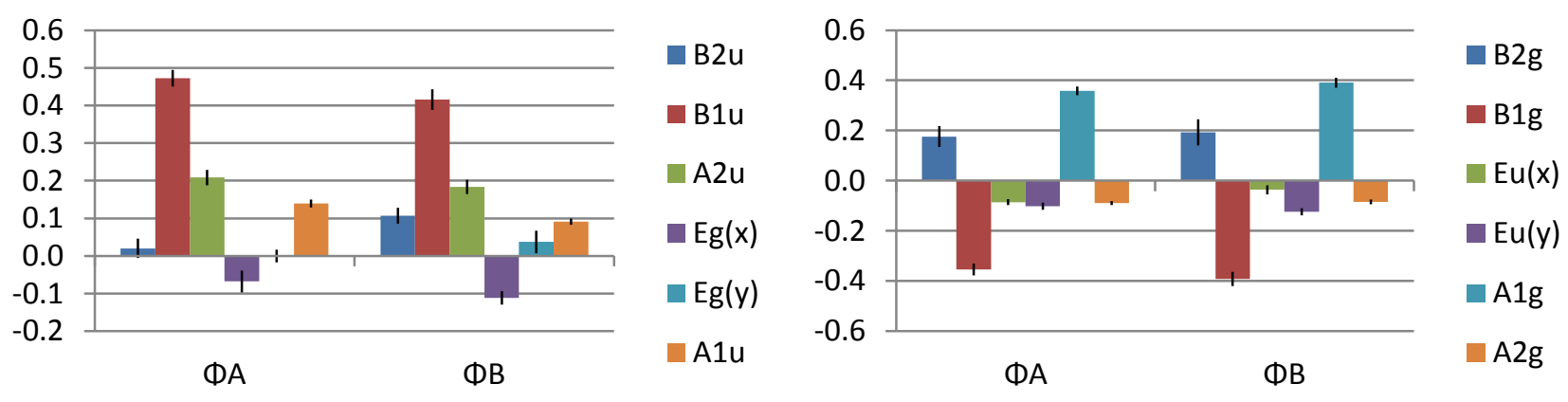

Figure 23: Mean minimum basis normal-coordinate displacements of each ETC cofactor in RBP4. Out-of-plane (left) and in-plane (right). Yaxes in angstroms; error bars indicate two standard errors and $n=16$.


Figure 24: Mean minimum basis normal-coordinate displacements of each ETC cofactor in RBP5. Out-of-plane (left) and in-plane (right). Yaxes in angstroms; error bars indicate two standard errors and $n=10$. 
5.5.4 Blastochloris viridis bacteriopheophytins

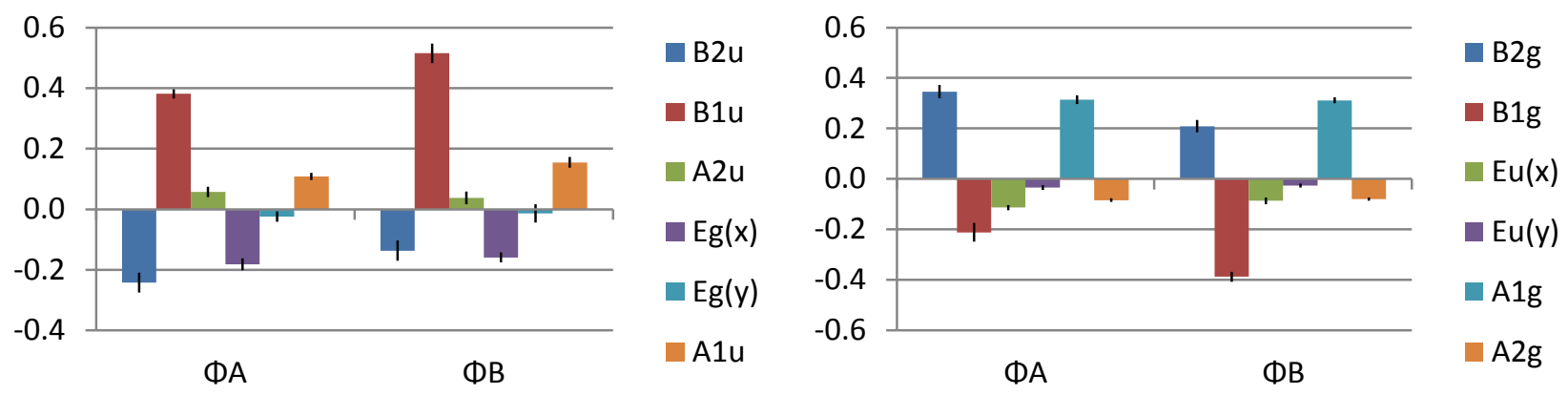

Figure 25: Mean minimum basis normal-coordinate displacements of each ETC cofactor in BBP1. Out-of-plane (left) and in-plane (right). Yaxes in angstroms; error bars indicate two standard errors and $\mathrm{n}=13$.
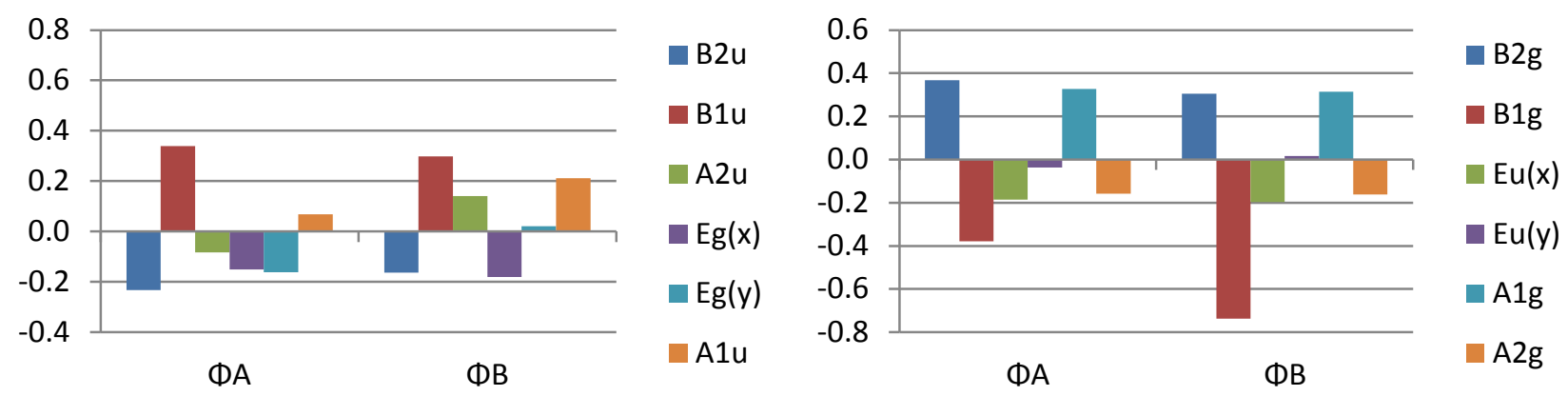

Figure 26: Minimum basis normal-coordinate displacements of each ETC cofactor in 3D38. Out-of-plane (left) and in-plane (right). Y-axes in angstroms.

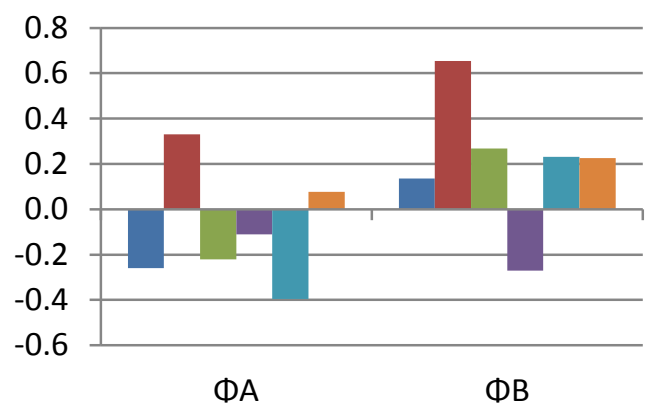

B2u
B1u
$A 2 u$
Eg(x)
Eg(y)
A1u

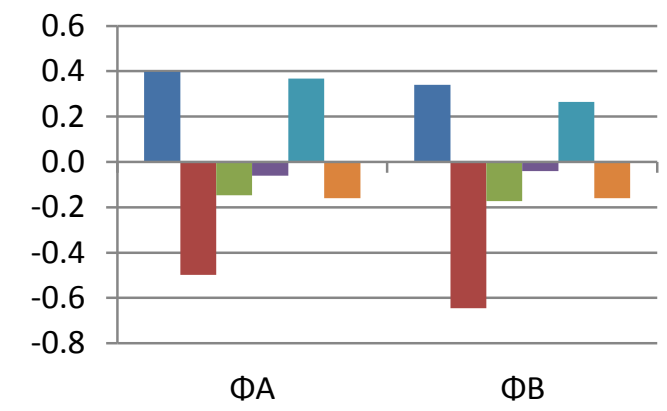

B2 $\mathrm{g}$

B1g

Eu(x)

- Eu(y)

A1g

A2g

Figure 27: Minimum basis normal-coordinate displacements of each ETC cofactor in 3G7F. Out-of-plane (left) and in-plane (right). Y-axes in angstroms. 


\subsection{Skeletal Deviation Plots: Local Nonplanarity}

\subsubsection{Rhodobacter sphaeroides bacteriochlorophylls}

\section{Skeletal Deviation Plots of Averaged Conformations (RBC3)}


Figure 28: Skeletal plots of z-axis displacements from the NSD minimum (blue) and extended basis (red) simulation of re-centred RBC3. BA (top left), BB (top right), DL (bottom left) and DM (bottom right). Y-axes in angstroms.
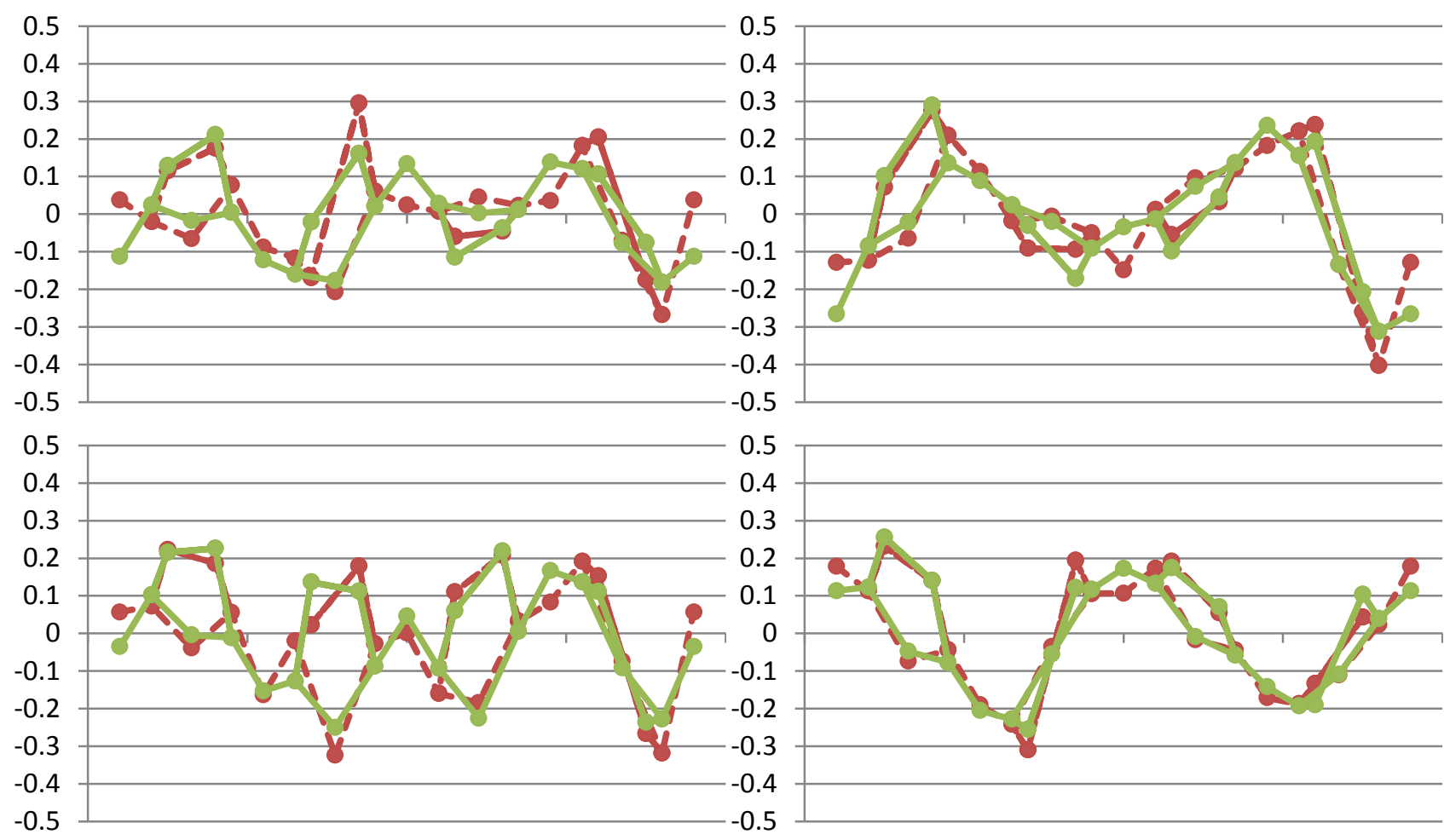

Figure 29: Skeletal plots of z-axis displacements from the NSD extended basis simulation (green) and complete basis, i.e. the observed structure, (red) of re-centred RBC3. BA (top left), BB (top right), DL (bottom left) and DM (bottom right). Y-axes in angstroms. 


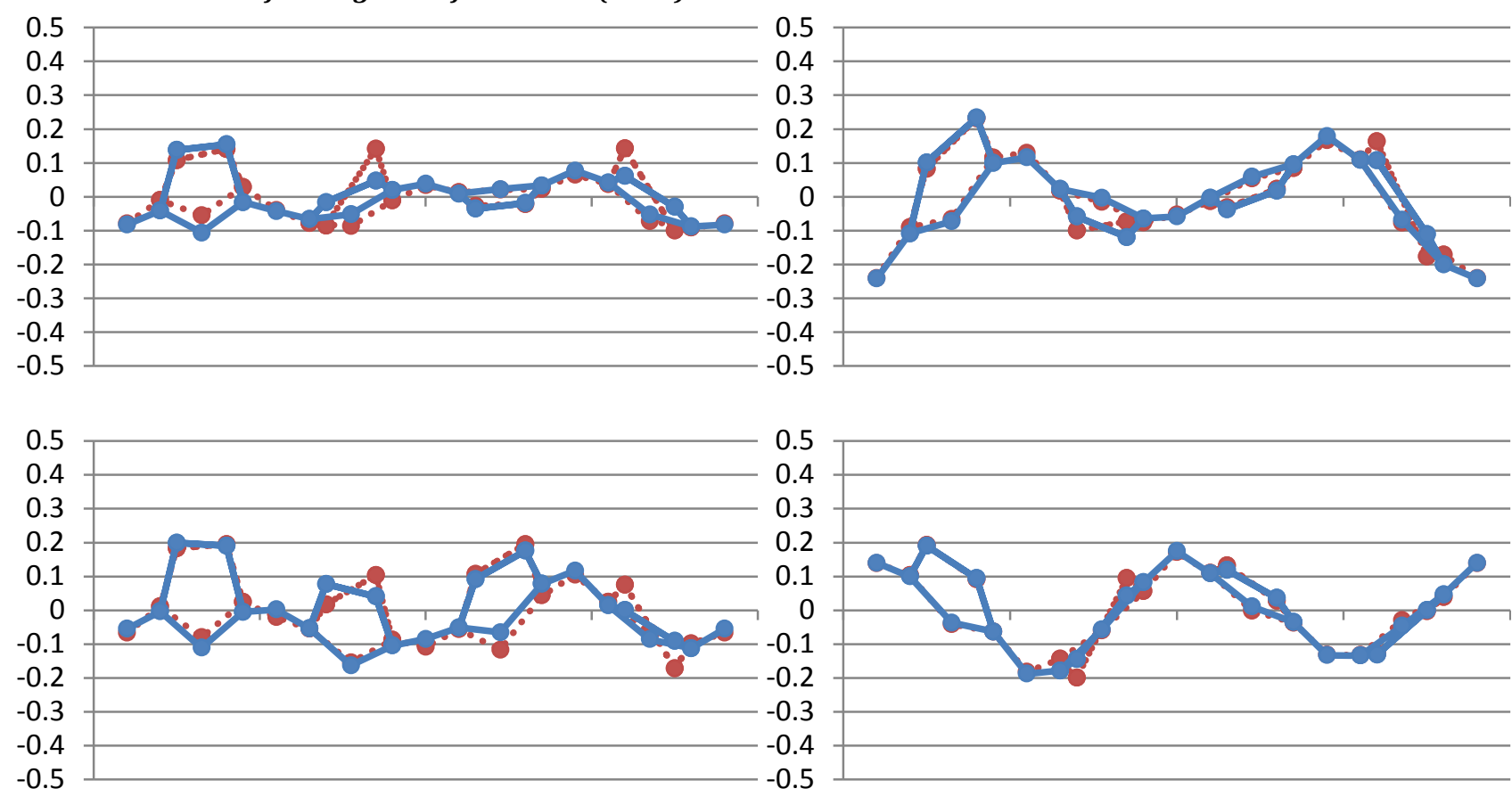

Figure 30: Comparison of minimum basis simulated (blue), with the observed (red), Z-axis displacements of: BA (top left), BB (top right), DL (bottom left) and DM (bottom right); axes in angstroms.

\subsubsection{Blastochloris viridis bacteriochlorophylls}
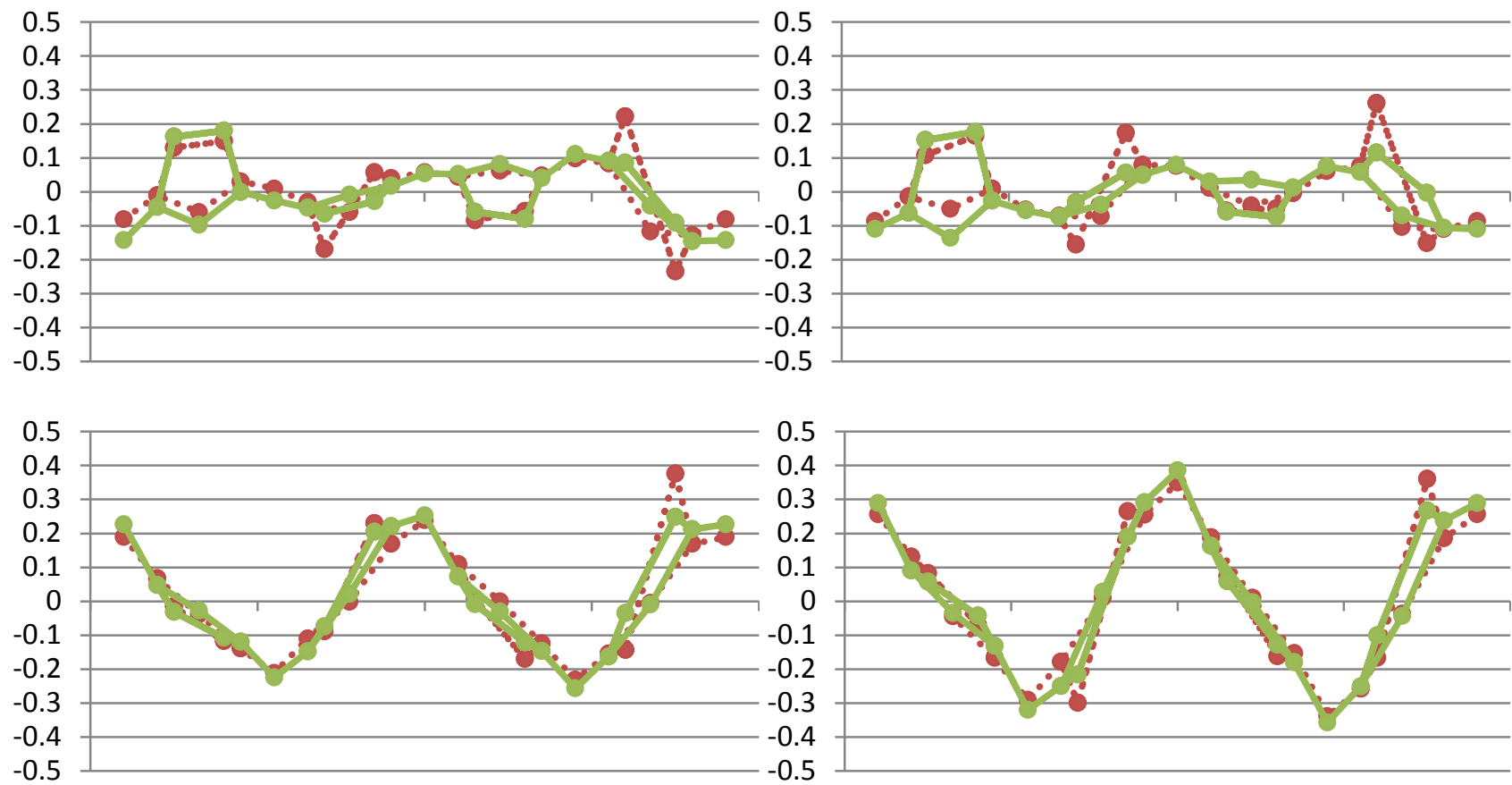

Figure 31: Comparison of the extended basis simulated (green) Z-axis distortions with those that are observed (red) for: BA (top left), BB (top right), DL (bottom left) and DM (bottom right). Y-Axes / angstroms 


\subsubsection{Rhodobacter sphaeroides bacteriopheophytins}

\section{Skeletal deviation plots (RBP4)}
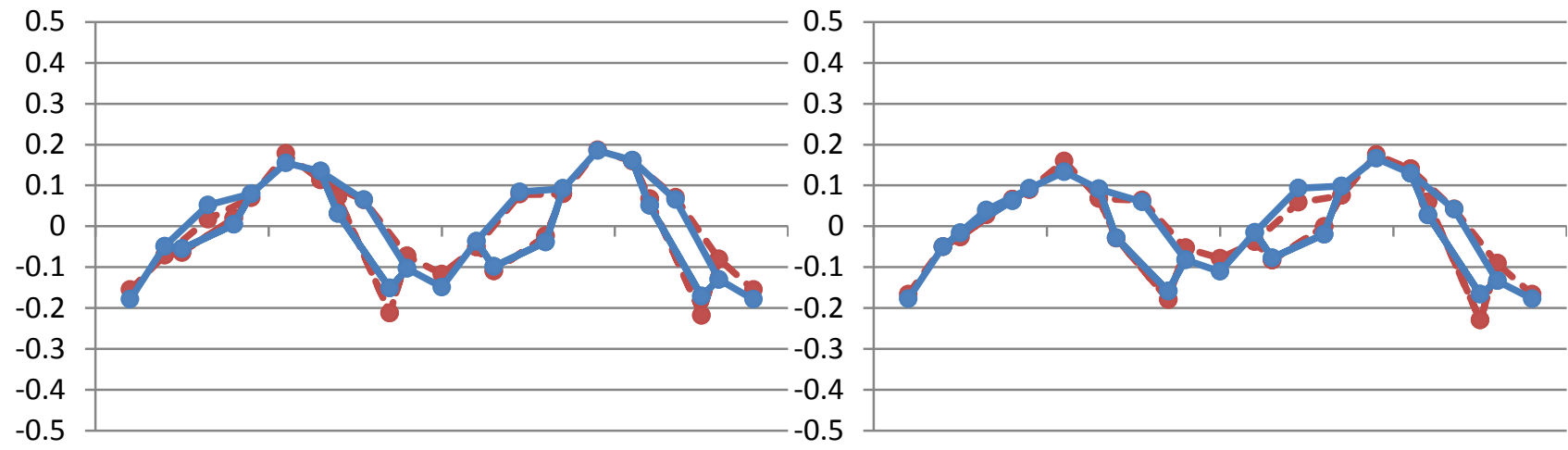

Figure 32: Comparison of the minimum basis simulated (blue) Z-axis distortions with those that are observed (red) for: ФA (left) and ФB (right). Y-Axes / angstroms.

\section{Skeletal deviation plots (RBP5)}
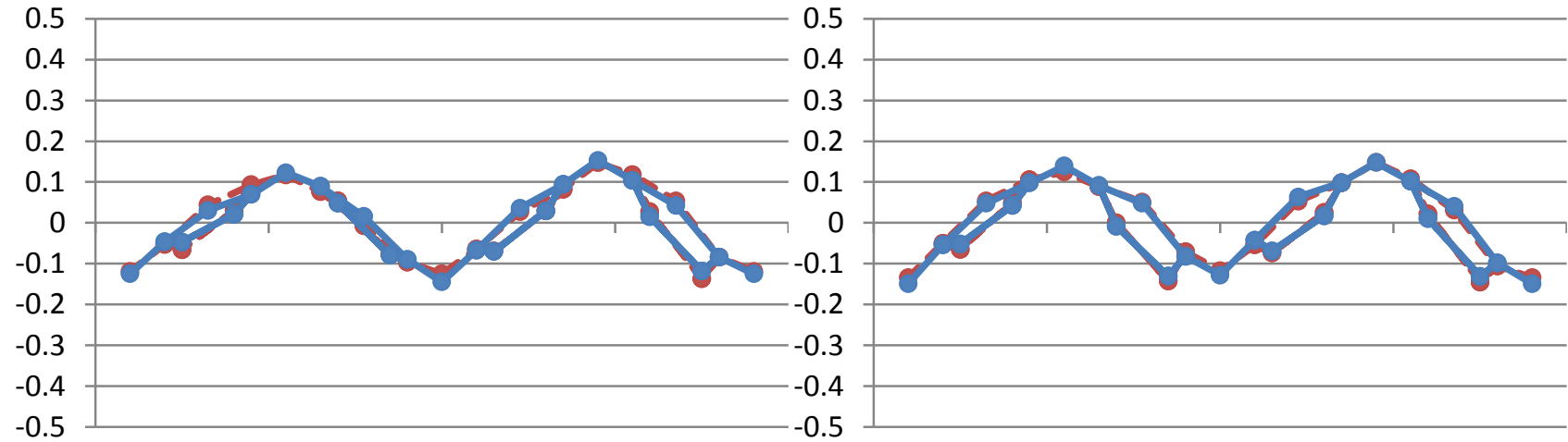

Figure 33: Comparison of the minimum basis simulated (blue) Z-axis distortions with those that are observed (red) for: $Ф A$ (left) and $Ф B$ (right). Y-Axes / angstroms.

\subsubsection{Blastochloris viridis bacteriopheophytins}

\section{Skeletal plots (BBP1)}
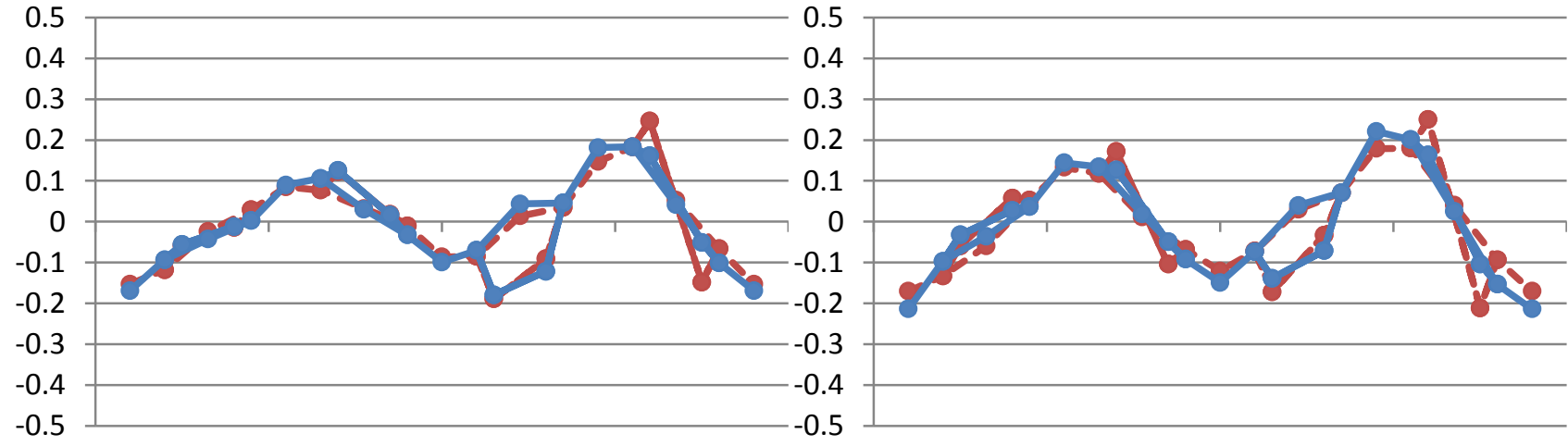

Figure 34: Comparison of the minimum basis simulated (blue) Z-axis distortions with those that are observed (red) for: ФA (left) and ФB (right). Y-Axes / angstroms. 


\subsection{Tabular NSD descriptive statistics}

\subsubsection{Rhodobacter sphaeroides bacteriochlorophylls - RBC2}

Table 11: Descriptive statistics of minimum basis out-of-plane parameters and normal-coordinate displacements for each ETC cofactor in all RBC2a experiments. $\mathrm{n}=8$.

\begin{tabular}{|c|c|c|c|c|c|c|c|c|}
\hline & BA & BB & $\mathrm{DL}$ & $\mathrm{DM}$ & $B A$ & BB & $\mathrm{DL}$ & DM \\
\hline & \multicolumn{4}{|c|}{ Doop } & \multicolumn{4}{|c|}{ Dip } \\
\hline MEAN & 0.0948 & 0.1851 & 0.1766 & 0.1122 & 0.3096 & 0.3686 & 0.4009 & 0.3937 \\
\hline SSD & 0.0156 & 0.0869 & 0.0372 & 0.0481 & 0.0511 & 0.0453 & 0.0478 & 0.0391 \\
\hline \multirow[t]{2}{*}{ SE } & 0.0055 & 0.0307 & 0.0132 & 0.0170 & 0.0181 & 0.0160 & 0.0169 & 0.0138 \\
\hline & \multicolumn{4}{|c|}{ 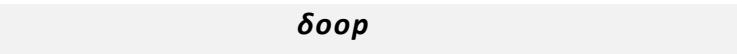 } & \multicolumn{4}{|c|}{ Sip } \\
\hline MEAN & 0.0061 & 0.0110 & 0.0045 & 0.0032 & 0.0588 & 0.0683 & 0.0621 & 0.0643 \\
\hline SSD & 0.0011 & 0.0111 & 0.0010 & 0.0006 & 0.0018 & 0.0157 & 0.0020 & 0.0027 \\
\hline \multirow[t]{2}{*}{ SE } & 0.0004 & 0.0039 & 0.0004 & 0.0002 & 0.0006 & 0.0056 & 0.0007 & 0.0009 \\
\hline & \multicolumn{4}{|c|}{$B 2 u$} & \multicolumn{4}{|c|}{$B 2 g$} \\
\hline MEAN & -0.0102 & -0.0737 & 0.0895 & 0.0707 & -0.1075 & -0.1260 & -0.0501 & -0.0392 \\
\hline SSD & 0.0376 & 0.1481 & 0.1064 & 0.0721 & 0.0367 & 0.0564 & 0.0718 & 0.0288 \\
\hline \multirow[t]{2}{*}{ SE } & 0.0133 & 0.0524 & 0.0376 & 0.0255 & 0.0130 & 0.0199 & 0.0254 & 0.0102 \\
\hline & \multicolumn{4}{|c|}{ B1u } & \multicolumn{4}{|c|}{ B1g } \\
\hline MEAN & 0.0393 & 0.0285 & 0.0501 & -0.0613 & -0.0127 & -0.0419 & -0.0933 & -0.1664 \\
\hline SSD & 0.0181 & 0.0655 & 0.0498 & 0.0267 & 0.0350 & 0.0410 & 0.0888 & 0.0291 \\
\hline \multirow[t]{2}{*}{ SE } & 0.0064 & 0.0232 & 0.0176 & 0.0094 & 0.0124 & 0.0145 & 0.0314 & 0.0103 \\
\hline & \multicolumn{4}{|c|}{$A 2 u$} & \multicolumn{4}{|c|}{$E u(x)$} \\
\hline MEAN & -0.0640 & -0.0679 & -0.0851 & -0.0146 & -0.1636 & -0.1513 & -0.1373 & -0.1664 \\
\hline SSD & 0.0124 & 0.0447 & 0.0256 & 0.0139 & 0.0253 & 0.0789 & 0.0678 & 0.0232 \\
\hline \multirow[t]{2}{*}{ SE } & 0.0044 & 0.0158 & 0.0091 & 0.0049 & 0.0089 & 0.0279 & 0.0240 & 0.0082 \\
\hline & \multicolumn{4}{|c|}{$E g(x)$} & \multicolumn{4}{|c|}{$E u(y)$} \\
\hline MEAN & -0.0343 & -0.0088 & -0.0297 & -0.0126 & 0.0039 & -0.0189 & -0.0005 & 0.0561 \\
\hline SSD & 0.0140 & 0.0294 & 0.0206 & 0.0083 & 0.0335 & 0.0533 & 0.0729 & 0.0221 \\
\hline \multirow[t]{2}{*}{ SE } & 0.0049 & 0.0104 & 0.0073 & 0.0029 & 0.0118 & 0.0189 & 0.0258 & 0.0078 \\
\hline & \multicolumn{4}{|c|}{$E g(y)$} & \multicolumn{4}{|c|}{$A 1 g$} \\
\hline MEAN & 0.0187 & 0.0000 & -0.0248 & 0.0020 & 0.2261 & 0.2850 & 0.3273 & 0.2989 \\
\hline SSD & 0.0109 & 0.0406 & 0.0078 & 0.0048 & 0.0641 & 0.0553 & 0.0637 & 0.0608 \\
\hline \multirow[t]{2}{*}{ SE } & 0.0039 & 0.0144 & 0.0027 & 0.0017 & 0.0227 & 0.0196 & 0.0225 & 0.0215 \\
\hline & \multicolumn{4}{|c|}{$A 1 u$} & \multicolumn{4}{|c|}{$A 2 g$} \\
\hline MEAN & 0.0003 & 0.0303 & -0.0069 & -0.0058 & -0.0337 & -0.0242 & -0.0432 & -0.0370 \\
\hline SSD & 0.0031 & 0.0492 & 0.0031 & 0.0052 & 0.0068 & 0.0112 & 0.0059 & 0.0027 \\
\hline SE & 0.0011 & 0.0174 & 0.0011 & 0.0018 & 0.0024 & 0.0040 & 0.0021 & 0.0010 \\
\hline
\end{tabular}

Table 12: Descriptive statistics of minimum basis out-of-plane parameters and normal-coordinate displacements for each ETC cofactor in all RBC2b experiments. $\mathrm{n}=10$.

\begin{tabular}{|c|c|c|c|c|c|c|c|c|}
\hline & BA & $\mathrm{BB}$ & $\mathrm{DL}$ & DM & $\mathrm{BA}$ & BB & $\mathrm{DL}$ & $\mathrm{DM}$ \\
\hline & \multicolumn{4}{|c|}{ Doop } & \multicolumn{4}{|c|}{ Dip } \\
\hline MEAN & 0.2903 & 0.3159 & 0.3275 & 0.2980 & 0.3143 & 0.3003 & 0.3888 & 0.3947 \\
\hline SSD & 0.0629 & 0.0604 & 0.0586 & 0.0712 & 0.0449 & 0.0432 & 0.0367 & 0.0239 \\
\hline \multirow[t]{2}{*}{ SE } & 0.0199 & 0.0191 & 0.0185 & 0.0225 & 0.0142 & 0.0137 & 0.0116 & 0.0076 \\
\hline & \multicolumn{4}{|c|}{ Soop } & \multicolumn{4}{|c|}{ sip } \\
\hline MEAN & 0.0238 & 0.0228 & 0.0188 & 0.0142 & 0.0687 & 0.0684 & 0.0661 & 0.0701 \\
\hline SSD & 0.0070 & 0.0062 & 0.0053 & 0.0026 & 0.0046 & 0.0021 & 0.0016 & 0.0012 \\
\hline
\end{tabular}




\begin{tabular}{|c|c|c|c|c|c|c|c|c|}
\hline & $\mathrm{BA}$ & BB & $\mathrm{DL}$ & $\mathrm{DM}$ & $B A$ & BB & $\mathrm{DL}$ & $\mathrm{DM}$ \\
\hline \multirow[t]{2}{*}{ SE } & 0.0022 & 0.0020 & 0.0017 & 0.0008 & 0.0014 & 0.0007 & 0.0005 & 0.0004 \\
\hline & \multicolumn{4}{|c|}{$B 2 u$} & \multicolumn{4}{|c|}{$B 2 g$} \\
\hline MEAN & -0.0468 & 0.0006 & 0.1391 & 0.1049 & 0.0655 & 0.0753 & 0.0719 & 0.1090 \\
\hline SSD & 0.0878 & 0.0884 & 0.0841 & 0.0865 & 0.0163 & 0.0163 & 0.0232 & 0.0281 \\
\hline \multirow[t]{2}{*}{ SE } & 0.0278 & 0.0280 & 0.0266 & 0.0273 & 0.0051 & 0.0052 & 0.0073 & 0.0089 \\
\hline & \multicolumn{4}{|c|}{$B 1 u$} & \multicolumn{4}{|c|}{$B 1 g$} \\
\hline MEAN & -0.0192 & 0.0937 & 0.0614 & -0.1933 & -0.1634 & -0.1652 & -0.2160 & -0.2355 \\
\hline SSD & 0.0175 & 0.0501 & 0.0328 & 0.0594 & 0.0396 & 0.0372 & 0.0199 & 0.0286 \\
\hline \multirow[t]{2}{*}{ SE } & 0.0055 & 0.0159 & 0.0104 & 0.0188 & 0.0125 & 0.0117 & 0.0063 & 0.0090 \\
\hline & \multicolumn{4}{|c|}{$A 2 u$} & \multicolumn{4}{|c|}{$E u(x)$} \\
\hline MEAN & -0.2114 & -0.2212 & -0.2472 & -0.1426 & -0.0519 & -0.0261 & -0.0456 & -0.0526 \\
\hline SSD & 0.0367 & 0.0443 & 0.0558 & 0.0099 & 0.0236 & 0.0382 & 0.0165 & 0.0240 \\
\hline \multirow[t]{2}{*}{ SE } & 0.0116 & 0.0140 & 0.0176 & 0.0031 & 0.0075 & 0.0121 & 0.0052 & 0.0076 \\
\hline & \multicolumn{4}{|c|}{$E g(x)$} & \multicolumn{4}{|c|}{$E u(y)$} \\
\hline MEAN & -0.1473 & -0.1332 & -0.1148 & -0.0847 & -0.0552 & -0.0641 & -0.1046 & -0.0493 \\
\hline SSD & 0.0270 & 0.0504 & 0.0339 & 0.0135 & 0.0385 & 0.0413 & 0.0370 & 0.0339 \\
\hline \multirow[t]{2}{*}{ SE } & 0.0085 & 0.0159 & 0.0107 & 0.0043 & 0.0122 & 0.0131 & 0.0117 & 0.0107 \\
\hline & \multicolumn{4}{|c|}{$E g(y)$} & \multicolumn{4}{|c|}{ A1g } \\
\hline MEAN & 0.0713 & 0.1134 & 0.0121 & 0.0646 & 0.2266 & 0.1956 & 0.2717 & 0.2678 \\
\hline SSD & 0.0547 & 0.0296 & 0.0288 & 0.0218 & 0.0317 & 0.0455 & 0.0431 & 0.0333 \\
\hline \multirow[t]{2}{*}{ SE } & 0.0173 & 0.0094 & 0.0091 & 0.0069 & 0.0100 & 0.0144 & 0.0136 & 0.0105 \\
\hline & \multicolumn{4}{|c|}{ A1u } & \multicolumn{4}{|c|}{$A 2 g$} \\
\hline MEAN & -0.0378 & -0.0055 & -0.0260 & -0.0511 & -0.0897 & -0.0980 & -0.0989 & -0.0896 \\
\hline SSD & 0.0264 & 0.0251 & 0.0165 & 0.0130 & 0.0110 & 0.0073 & 0.0018 & 0.0062 \\
\hline SE & 0.0084 & 0.0079 & 0.0052 & 0.0041 & 0.0035 & 0.0023 & 0.0006 & 0.0020 \\
\hline
\end{tabular}

5.7.2 Rhodobacter sphaeroides bacteriochlorophylls - RBC3

Table 13: Descriptive statistics of minimum basis out-of-plane parameters and normal-coordinate displacements for each ETC cofactor in all RBC3 experiments. $n=16$.

\begin{tabular}{|c|c|c|c|c|c|c|c|c|}
\hline & BA & BB & $\mathrm{DL}$ & DM & BA & BB & $\mathrm{DL}$ & DM \\
\hline & \multicolumn{4}{|c|}{ Doop } & \multicolumn{4}{|c|}{ Dip } \\
\hline MEAN & 0.4995 & 0.7487 & 0.6498 & 0.7200 & 0.2698 & 0.3092 & 0.2952 & 0.3108 \\
\hline SSD & 0.0900 & 0.0756 & 0.0578 & 0.0890 & 0.0322 & 0.0387 & 0.0393 & 0.0285 \\
\hline \multirow[t]{2}{*}{ SE } & 0.0225 & 0.0189 & 0.0145 & 0.0223 & 0.0080 & 0.0097 & 0.0098 & 0.0071 \\
\hline & \multicolumn{4}{|c|}{ Soop } & \multicolumn{4}{|c|}{ Sip } \\
\hline MEAN & 0.0946 & 0.0737 & 0.0979 & 0.0666 & 0.0506 & 0.0528 & 0.0539 & 0.0556 \\
\hline SSD & 0.0237 & 0.0233 & 0.0168 & 0.0249 & 0.0059 & 0.0053 & 0.0057 & 0.0050 \\
\hline \multirow[t]{2}{*}{ SE } & 0.0059 & 0.0058 & 0.0042 & 0.0062 & 0.0015 & 0.0013 & 0.0014 & 0.0012 \\
\hline & \multicolumn{4}{|c|}{$B 2 u$} & \multicolumn{4}{|c|}{$B 2 g$} \\
\hline MEAN & 0.0604 & 0.2486 & 0.2418 & 0.3382 & 0.0661 & 0.0866 & 0.0326 & 0.0180 \\
\hline SSD & 0.0760 & 0.1230 & 0.0874 & 0.0596 & 0.0403 & 0.0729 & 0.0669 & 0.0493 \\
\hline \multirow[t]{2}{*}{ SE } & 0.0190 & 0.0307 & 0.0218 & 0.0149 & 0.0101 & 0.0182 & 0.0167 & 0.0123 \\
\hline & \multicolumn{4}{|c|}{ B1u } & \multicolumn{4}{|c|}{ B1g } \\
\hline MEAN & 0.0387 & 0.5398 & 0.1404 & -0.5483 & -0.1934 & -0.2099 & -0.2247 & -0.2478 \\
\hline SSD & 0.0579 & 0.0946 & 0.1019 & 0.1179 & 0.0698 & 0.0370 & 0.0386 & 0.0441 \\
\hline \multirow[t]{2}{*}{ SE } & 0.0145 & 0.0237 & 0.0255 & 0.0295 & 0.0174 & 0.0093 & 0.0097 & 0.0110 \\
\hline & \multicolumn{4}{|c|}{$A 2 u$} & \multicolumn{4}{|c|}{$E u(x)$} \\
\hline MEAN & -0.1881 & -0.0672 & -0.4238 & -0.1913 & -0.0562 & -0.0115 & -0.0627 & -0.0681 \\
\hline
\end{tabular}




\begin{tabular}{|c|c|c|c|c|c|c|c|c|}
\hline & $B A$ & BB & $\mathrm{DL}$ & $\mathrm{DM}$ & BA & BB & $\mathrm{DL}$ & $\mathrm{DM}$ \\
\hline SSD & 0.0747 & 0.0983 & 0.0746 & 0.0675 & 0.0460 & 0.0424 & 0.0358 & 0.0288 \\
\hline \multirow[t]{2}{*}{ SE } & 0.0187 & 0.0246 & 0.0186 & 0.0169 & 0.0115 & 0.0106 & 0.0089 & 0.0072 \\
\hline & \multicolumn{4}{|c|}{$E g(x)$} & \multicolumn{4}{|c|}{$E u(y)$} \\
\hline MEAN & -0.4214 & -0.3613 & -0.1966 & -0.1219 & -0.0101 & -0.1067 & -0.0800 & -0.0217 \\
\hline SSD & 0.0761 & 0.0859 & 0.0596 & 0.0622 & 0.0298 & 0.0528 & 0.0296 & 0.0381 \\
\hline \multirow[t]{2}{*}{ SE } & 0.0190 & 0.0215 & 0.0149 & 0.0156 & 0.0074 & 0.0132 & 0.0074 & 0.0095 \\
\hline & \multicolumn{4}{|c|}{$E g(y)$} & \multicolumn{4}{|c|}{ A1g } \\
\hline MEAN & -0.0684 & 0.1348 & -0.2822 & 0.0270 & 0.0109 & -0.0621 & 0.0159 & 0.0411 \\
\hline SSD & 0.1190 & 0.0696 & 0.0614 & 0.0655 & 0.0727 & 0.0618 & 0.0736 & 0.0870 \\
\hline \multirow[t]{2}{*}{ SE } & 0.0297 & 0.0174 & 0.0154 & 0.0164 & 0.0182 & 0.0154 & 0.0184 & 0.0217 \\
\hline & \multicolumn{4}{|c|}{$A 1 u$} & \multicolumn{4}{|c|}{$A 2 g$} \\
\hline MEAN & -0.0023 & 0.1053 & 0.1342 & -0.1601 & -0.1205 & -0.1252 & -0.1163 & -0.1230 \\
\hline SSD & 0.0435 & 0.0758 & 0.0495 & 0.0708 & 0.0161 & 0.0175 & 0.0160 & 0.0211 \\
\hline SE & 0.0109 & 0.0190 & 0.0124 & 0.0177 & 0.0040 & 0.0044 & 0.0040 & 0.0053 \\
\hline
\end{tabular}

5.7.3 Rhodobacter sphaeroides bacteriochlorophylls - RBC4

Table 14: Descriptive statistics of minimum basis out-of-plane parameters and normal-coordinate displacements for each ETC cofactor in all RBC4 experiments. $\mathrm{n}=32$.

\begin{tabular}{|c|c|c|c|c|c|c|c|c|}
\hline & $B A$ & BB & $\mathrm{DL}$ & DM & $B A$ & BB & $\mathrm{DL}$ & DM \\
\hline & \multicolumn{4}{|c|}{ Doop } & \multicolumn{4}{|c|}{ Dip } \\
\hline MEAN & 0.3611 & 0.5848 & 0.5168 & 0.5694 & 0.3052 & 0.3894 & 0.4031 & 0.3704 \\
\hline SSD & 0.0688 & 0.1244 & 0.0677 & 0.0824 & 0.0740 & 0.0692 & 0.0609 & 0.0735 \\
\hline \multirow[t]{2}{*}{ SE } & 0.0122 & 0.0220 & 0.0120 & 0.0146 & 0.0131 & 0.0122 & 0.0108 & 0.0130 \\
\hline & \multicolumn{4}{|c|}{ Soop } & \multicolumn{4}{|c|}{ sip } \\
\hline MEAN & 0.0340 & 0.0255 & 0.0349 & 0.0205 & 0.0498 & 0.0519 & 0.0499 & 0.0546 \\
\hline SSD & 0.0153 & 0.0144 & 0.0155 & 0.0116 & 0.0050 & 0.0053 & 0.0046 & 0.0044 \\
\hline \multirow[t]{2}{*}{ SE } & 0.0027 & 0.0025 & 0.0027 & 0.0020 & 0.0009 & 0.0009 & 0.0008 & 0.0008 \\
\hline & \multicolumn{4}{|c|}{$B 2 u$} & \multicolumn{4}{|c|}{$B 2 g$} \\
\hline MEAN & 0.0691 & 0.1940 & 0.2457 & 0.2630 & 0.0358 & 0.0341 & -0.0261 & 0.0021 \\
\hline SSD & 0.0961 & 0.0702 & 0.0757 & 0.1078 & 0.0973 & 0.0693 & 0.0799 & 0.0609 \\
\hline \multirow[t]{2}{*}{ SE } & 0.0170 & 0.0124 & 0.0134 & 0.0191 & 0.0172 & 0.0122 & 0.0141 & 0.0108 \\
\hline & \multicolumn{4}{|c|}{ B1u } & \multicolumn{4}{|c|}{ B1g } \\
\hline MEAN & 0.0551 & 0.4220 & 0.1835 & -0.4504 & -0.1219 & -0.2318 & -0.2237 & -0.2019 \\
\hline SSD & 0.0451 & 0.1220 & 0.0678 & 0.0808 & 0.0956 & 0.1012 & 0.0992 & 0.0874 \\
\hline \multirow[t]{2}{*}{ SE } & 0.0080 & 0.0216 & 0.0120 & 0.0143 & 0.0169 & 0.0179 & 0.0175 & 0.0154 \\
\hline & \multicolumn{4}{|c|}{$A 2 u$} & \multicolumn{4}{|c|}{$E u(x)$} \\
\hline MEAN & -0.1472 & -0.0656 & -0.3326 & -0.1012 & -0.0713 & -0.0463 & -0.0785 & -0.0559 \\
\hline SSD & 0.1008 & 0.0953 & 0.0673 & 0.0858 & 0.0520 & 0.0436 & 0.0318 & 0.0450 \\
\hline \multirow[t]{2}{*}{ SE } & 0.0178 & 0.0169 & 0.0119 & 0.0152 & 0.0092 & 0.0077 & 0.0056 & 0.0079 \\
\hline & \multicolumn{4}{|c|}{$E g(x)$} & \multicolumn{4}{|c|}{$E u(y)$} \\
\hline MEAN & -0.2699 & -0.2761 & -0.0951 & -0.1004 & -0.0323 & -0.1035 & -0.0684 & -0.0335 \\
\hline SSD & 0.0739 & 0.0682 & 0.0672 & 0.0620 & 0.0370 & 0.0377 & 0.0253 & 0.0483 \\
\hline \multirow[t]{2}{*}{ SE } & 0.0131 & 0.0121 & 0.0119 & 0.0110 & 0.0065 & 0.0067 & 0.0045 & 0.0085 \\
\hline & \multicolumn{4}{|c|}{$E g(y)$} & \multicolumn{4}{|c|}{ A1g } \\
\hline MEAN & 0.0001 & 0.1349 & -0.1625 & -0.0228 & 0.2122 & 0.2472 & 0.2754 & 0.2702 \\
\hline SSD & 0.0729 & 0.0842 & 0.1054 & 0.0522 & 0.0558 & 0.0642 & 0.0686 & 0.0632 \\
\hline SE & 0.0129 & 0.0149 & 0.0186 & 0.0092 & 0.0099 & 0.0113 & 0.0121 & 0.0112 \\
\hline
\end{tabular}




\begin{tabular}{lccccccccc}
\hline & BA & BB & DL & DM & BA & BB & DL & DM \\
\hline \multirow{2}{*}{ MEAN } & -0.0022 & 0.0451 & 0.0253 & -0.0689 & -0.0766 & -0.0729 & -0.0796 & -0.0685 \\
SSD & 0.0383 & 0.0342 & 0.0348 & 0.0463 & 0.0164 & 0.0185 & 0.0224 & 0.0262 \\
SE & 0.0068 & 0.0060 & 0.0061 & 0.0082 & 0.0029 & 0.0033 & 0.0040 & 0.0046 \\
\hline
\end{tabular}

\subsubsection{Blastochloris viridis bacteriochlorophylls - 1PRC}

\section{Minimum Basis}

Table 15: Minimum basis out-of-plane parameters and normal-coordinate displacements for each ETC cofactor in 1PRC.

\begin{tabular}{|c|c|c|c|c|c|c|c|}
\hline BA & BB & $\mathrm{DL}$ & DM & BA & BB & $\mathrm{DL}$ & DM \\
\hline \multicolumn{4}{|c|}{ Doop } & \multicolumn{4}{|c|}{ Dip } \\
\hline 0.3019 & 0.1875 & 0.4142 & 0.663 & 0.2998 & 0.3533 & 0.3796 & 0.2809 \\
\hline \multicolumn{4}{|c|}{ Soop } & \multicolumn{4}{|c|}{ sip } \\
\hline 0.0394 & 0.0458 & 0.0401 & 0.0518 & 0.0472 & 0.0492 & 0.0515 & 0.0521 \\
\hline \multicolumn{4}{|c|}{$B 2 u$} & \multicolumn{4}{|c|}{$B 2 g$} \\
\hline 0.1285 & 0.0619 & -0.154 & 0.1016 & 0.0674 & 0.0859 & 0.0714 & 0.0714 \\
\hline \multicolumn{4}{|c|}{ B1u } & \multicolumn{4}{|c|}{$B 1 g$} \\
\hline 0.1168 & 0.0503 & -0.3335 & -0.6127 & -0.1271 & -0.1604 & -0.2989 & -0.0871 \\
\hline \multicolumn{4}{|c|}{$A 2 u$} & \multicolumn{4}{|c|}{$E u(x)$} \\
\hline-0.0856 & 0.0065 & -0.0628 & -0.0368 & -0.0911 & -0.1037 & -0.1082 & -0.0834 \\
\hline \multicolumn{4}{|c|}{$E g(x)$} & \multicolumn{4}{|c|}{$E u(y)$} \\
\hline-0.2169 & -0.1562 & -0.0963 & 0.0465 & -0.0692 & -0.0454 & -0.0525 & -0.2157 \\
\hline \multicolumn{4}{|c|}{$E g(y)$} & \multicolumn{4}{|c|}{ A1g } \\
\hline 0.0698 & 0.06 & 0.0452 & 0.0876 & 0.2218 & 0.2691 & 0.1565 & 0.0381 \\
\hline \multicolumn{4}{|c|}{$A 1 u$} & \multicolumn{4}{|c|}{$A 2 g$} \\
\hline 0.0412 & -0.0272 & -0.146 & -0.2064 & -0.083 & -0.0804 & -0.1035 & -0.1063 \\
\hline
\end{tabular}

\section{Extended Basis}

Table 16: Extended basis parameters and normal-coordinate displacements for each ETC cofactor in 1PRC.

\begin{tabular}{|c|c|c|c|c|c|c|c|c|}
\hline & BA & BB & $\mathrm{DL}$ & DM & BA & BB & $\mathrm{DL}$ & DM \\
\hline & \multicolumn{4}{|c|}{ Doop } & \multicolumn{4}{|c|}{ Dip } \\
\hline \multirow[t]{2}{*}{ N/A } & 0.3201 & 0.2294 & 0.4737 & 0.7232 & 0.3503 & 0.4072 & 0.4272 & 0.3634 \\
\hline & \multicolumn{4}{|c|}{ Soop } & \multicolumn{4}{|c|}{ sip } \\
\hline \multirow[t]{2}{*}{ N/A } & 0.0401 & 0.0443 & 0.0176 & 0.0145 & 0.0360 & 0.0332 & 0.0345 & 0.0314 \\
\hline & \multicolumn{4}{|c|}{$B 2 u$} & \multicolumn{4}{|c|}{$B 2 g$} \\
\hline $1^{\text {st }}$ & 0.1280 & 0.0627 & -0.1544 & 0.1016 & 0.0673 & 0.0853 & 0.0711 & 0.0703 \\
\hline \multirow[t]{2}{*}{$2^{\text {nd }}$} & -0.0093 & 0.0711 & -0.0282 & -0.0004 & 0.0271 & 0.1138 & -0.0451 & -0.0778 \\
\hline & \multicolumn{4}{|c|}{ B1u } & \multicolumn{4}{|c|}{$B 1 g$} \\
\hline $1^{\text {st }}$ & 0.1168 & 0.0503 & -0.3335 & -0.6127 & -0.1266 & -0.1601 & -0.2967 & -0.0844 \\
\hline \multirow[t]{2}{*}{$2^{\text {nd }}$} & -0.0607 & 0.0215 & 0.1784 & 0.2313 & -0.0411 & -0.0514 & -0.0484 & -0.0237 \\
\hline & \multicolumn{4}{|c|}{$A 2 u$} & \multicolumn{4}{|c|}{$E u(x)$} \\
\hline $1^{\text {st }}$ & -0.0857 & 0.0078 & -0.0636 & -0.0341 & -0.0910 & -0.1040 & -0.1094 & -0.0847 \\
\hline \multirow[t]{2}{*}{$2^{\text {nd }}$} & -0.0011 & 0.0228 & -0.0085 & 0.0537 & -0.0701 & -0.1047 & -0.0125 & -0.1323 \\
\hline & \multicolumn{4}{|c|}{$E g(x)$} & \multicolumn{4}{|c|}{$E u(y)$} \\
\hline $1^{\text {st }}$ & -0.2160 & -0.1544 & -0.0956 & 0.0480 & -0.0681 & -0.0442 & -0.0499 & -0.2148 \\
\hline $2^{\text {nd }}$ & 0.0673 & 0.0967 & 0.0244 & 0.0206 & 0.1532 & 0.1146 & 0.1754 & 0.1494 \\
\hline
\end{tabular}




\begin{tabular}{|c|c|c|c|c|c|c|c|c|}
\hline & BA & BB & $\mathrm{DL}$ & DM & $\mathrm{BA}$ & BB & $\mathrm{DL}$ & $\mathrm{DM}$ \\
\hline & \multicolumn{4}{|c|}{$E g(y)$} & \multicolumn{4}{|c|}{$A 1 g$} \\
\hline $1^{\text {st }}$ & 0.0700 & 0.0599 & 0.0454 & 0.0855 & 0.2228 & 0.2681 & 0.1576 & 0.0379 \\
\hline \multirow[t]{2}{*}{$2^{\text {nd }}$} & 0.0264 & 0.0150 & -0.0219 & -0.0252 & 0.0294 & 0.0271 & 0.0515 & 0.0771 \\
\hline & \multicolumn{4}{|c|}{ A1u } & \multicolumn{4}{|c|}{$A 2 g$} \\
\hline $1^{\text {st }}$ & 0.0412 & -0.0272 & -0.1460 & -0.2064 & -0.0811 & -0.0789 & -0.1017 & -0.1052 \\
\hline $2^{\text {nd }}$ & -0.0431 & 0.0254 & 0.1378 & 0.1618 & 0.0363 & 0.0192 & 0.0107 & 0.0050 \\
\hline
\end{tabular}

\subsubsection{Blastochloris viridis bacteriochlorophylls - BBC1}

\section{Minimum Basis}

Table 17: Descriptive statistics of minimum basis out-of-plane parameters and normal-coordinate displacements for each ETC cofactor in all BBC1 experiments; $\mathrm{n}=13$.

\begin{tabular}{|c|c|c|c|c|c|c|c|c|}
\hline & BA & BB & $\mathrm{DL}$ & DM & BA & BB & $\mathrm{DL}$ & DM \\
\hline & \multicolumn{4}{|c|}{ Doop } & \multicolumn{4}{|c|}{ Dip } \\
\hline MEAN & 0.4466 & 0.4269 & 0.7582 & 1.0145 & 0.4245 & 0.3956 & 0.3739 & 0.3259 \\
\hline SSD & 0.0619 & 0.0590 & 0.0794 & 0.0765 & 0.0337 & 0.0664 & 0.0399 & 0.0465 \\
\hline \multirow[t]{2}{*}{ SE } & 0.0109 & 0.0104 & 0.0140 & 0.0135 & 0.0060 & 0.0117 & 0.0071 & 0.0082 \\
\hline & \multicolumn{4}{|c|}{ Soop } & \multicolumn{4}{|c|}{ sip } \\
\hline MEAN & 0.0493 & 0.0528 & 0.0383 & 0.0415 & 0.0450 & 0.0453 & 0.0459 & 0.0437 \\
\hline SSD & 0.0084 & 0.0071 & 0.0067 & 0.0104 & 0.0059 & 0.0067 & 0.0072 & 0.0078 \\
\hline \multirow[t]{2}{*}{ SE } & 0.0015 & 0.0012 & 0.0012 & 0.0018 & 0.0010 & 0.0012 & 0.0013 & 0.0014 \\
\hline & \multicolumn{4}{|c|}{$B 2 u$} & \multicolumn{4}{|c|}{$B 2 g$} \\
\hline MEAN & 0.1173 & 0.0225 & -0.2394 & -0.0766 & 0.0803 & 0.0551 & 0.0568 & 0.0482 \\
\hline SSD & 0.0956 & 0.0802 & 0.0608 & 0.1066 & 0.0597 & 0.0379 & 0.0359 & 0.0609 \\
\hline \multirow[t]{2}{*}{ SE } & 0.0169 & 0.0142 & 0.0107 & 0.0188 & 0.0105 & 0.0067 & 0.0063 & 0.0108 \\
\hline & \multicolumn{4}{|c|}{$B 1 u$} & \multicolumn{4}{|c|}{$B 1 g$} \\
\hline MEAN & 0.1230 & 0.0359 & -0.6792 & -0.9567 & -0.2644 & -0.2521 & -0.2148 & -0.1793 \\
\hline SSD & 0.0408 & 0.0865 & 0.0748 & 0.0837 & 0.0600 & 0.0594 & 0.0431 & 0.0549 \\
\hline \multirow[t]{2}{*}{ SE } & 0.0072 & 0.0153 & 0.0132 & 0.0148 & 0.0106 & 0.0105 & 0.0076 & 0.0097 \\
\hline & \multicolumn{4}{|c|}{$\mathrm{A2u}$} & \multicolumn{4}{|c|}{$E u(x)$} \\
\hline MEAN & -0.0514 & -0.1636 & -0.0365 & -0.0416 & -0.1096 & -0.0781 & -0.1234 & -0.1188 \\
\hline SSD & 0.0685 & 0.0606 & 0.0693 & 0.0619 & 0.0759 & 0.0431 & 0.0331 & 0.0664 \\
\hline \multirow[t]{2}{*}{ SE } & 0.0121 & 0.0107 & 0.0122 & 0.0109 & 0.0134 & 0.0076 & 0.0059 & 0.0117 \\
\hline & \multicolumn{4}{|c|}{$E g(x)$} & \multicolumn{4}{|c|}{$E u(y)$} \\
\hline MEAN & -0.3750 & -0.3589 & 0.0070 & -0.0661 & -0.0703 & -0.0260 & -0.0328 & -0.0347 \\
\hline SSD & 0.0563 & 0.0672 & 0.0746 & 0.0589 & 0.0292 & 0.0230 & 0.0243 & 0.0250 \\
\hline \multirow[t]{2}{*}{ SE } & 0.0099 & 0.0119 & 0.0132 & 0.0104 & 0.0052 & 0.0041 & 0.0043 & 0.0044 \\
\hline & \multicolumn{4}{|c|}{$E g(y)$} & \multicolumn{4}{|c|}{ A1g } \\
\hline MEAN & 0.0677 & 0.0670 & 0.0655 & 0.1498 & 0.2568 & 0.2671 & 0.2493 & 0.1993 \\
\hline SSD & 0.0601 & 0.0517 & 0.0622 & 0.0554 & 0.0554 & 0.0514 & 0.0351 & 0.0432 \\
\hline \multirow[t]{2}{*}{ SE } & 0.0106 & 0.0091 & 0.0110 & 0.0098 & 0.0098 & 0.0091 & 0.0062 & 0.0076 \\
\hline & \multicolumn{4}{|c|}{$A 1 u$} & \multicolumn{4}{|c|}{$A 2 g$} \\
\hline MEAN & 0.0706 & 0.0113 & -0.1827 & -0.2380 & -0.0782 & -0.0792 & -0.0877 & -0.0762 \\
\hline SSD & 0.0244 & 0.0229 & 0.0348 & 0.0435 & 0.0161 & 0.0186 & 0.0141 & 0.0180 \\
\hline SE & 0.0043 & 0.0041 & 0.0061 & 0.0077 & 0.0028 & 0.0033 & 0.0025 & 0.0032 \\
\hline
\end{tabular}


Table 18: Descriptive statistics of extended basis out-of-plane parameters and lowest energy normal-coordinate displacements for each ETC cofactor in all BBC1 experiments; $n=13$.

\begin{tabular}{|c|c|c|c|c|c|c|c|c|}
\hline & $B A$ & BB & $\mathrm{DL}$ & DM & BA & BB & $\mathrm{DL}$ & DM \\
\hline & \multicolumn{4}{|c|}{ Doop } & \multicolumn{4}{|c|}{ Dip } \\
\hline MEAN & 0.4852 & 0.4897 & 0.7864 & 1.0385 & 0.4590 & 0.4333 & 0.4180 & 0.3760 \\
\hline SSD & 0.0749 & 0.0661 & 0.0787 & 0.0771 & 0.0378 & 0.0656 & 0.0396 & 0.0380 \\
\hline \multirow[t]{2}{*}{ SE } & 0.0132 & 0.0117 & 0.0139 & 0.0136 & 0.0067 & 0.0116 & 0.0070 & 0.0067 \\
\hline & \multicolumn{4}{|c|}{ Soop } & \multicolumn{4}{|c|}{ Sip } \\
\hline MEAN & 0.0375 & 0.0367 & 0.0220 & 0.0205 & 0.0283 & 0.0290 & 0.0275 & 0.0254 \\
\hline SSD & 0.0105 & 0.0099 & 0.0073 & 0.0084 & 0.0088 & 0.0091 & 0.0088 & 0.0084 \\
\hline \multirow[t]{2}{*}{ SE } & 0.0019 & 0.0017 & 0.0013 & 0.0015 & 0.0016 & 0.0016 & 0.0016 & 0.0015 \\
\hline & \multicolumn{4}{|c|}{$B 2 u$} & \multicolumn{4}{|c|}{$B 2 g$} \\
\hline MEAN & 0.1191 & 0.0234 & -0.2389 & -0.0765 & 0.0808 & 0.0560 & 0.0578 & 0.0489 \\
\hline SSD & 0.0967 & 0.0802 & 0.0611 & 0.1067 & 0.0597 & 0.0375 & 0.0360 & 0.0608 \\
\hline \multirow[t]{2}{*}{ SE } & 0.0171 & 0.0142 & 0.0108 & 0.0189 & 0.0106 & 0.0066 & 0.0064 & 0.0108 \\
\hline & \multicolumn{4}{|c|}{ B1u } & \multicolumn{4}{|c|}{ B1g } \\
\hline MEAN & 0.1230 & 0.0359 & -0.6792 & -0.9567 & -0.2646 & -0.2525 & -0.2150 & -0.1797 \\
\hline SSD & 0.0408 & 0.0865 & 0.0748 & 0.0837 & 0.0610 & 0.0600 & 0.0434 & 0.0551 \\
\hline \multirow[t]{2}{*}{ SE } & 0.0072 & 0.0153 & 0.0132 & 0.0148 & 0.0108 & 0.0106 & 0.0077 & 0.0097 \\
\hline & \multicolumn{4}{|c|}{$A 2 u$} & \multicolumn{4}{|c|}{$E u(x)$} \\
\hline MEAN & -0.0567 & -0.1664 & -0.0365 & -0.0421 & -0.1101 & -0.0787 & -0.1239 & -0.1193 \\
\hline SSD & 0.0682 & 0.0617 & 0.0698 & 0.0632 & 0.0761 & 0.0431 & 0.0331 & 0.0663 \\
\hline \multirow[t]{2}{*}{ SE } & 0.0121 & 0.0109 & 0.0123 & 0.0112 & 0.0134 & 0.0076 & 0.0059 & 0.0117 \\
\hline & \multicolumn{4}{|c|}{$E g(x)$} & \multicolumn{4}{|c|}{$E u(y)$} \\
\hline MEAN & -0.3728 & -0.3527 & 0.0054 & -0.0670 & -0.0686 & -0.0245 & -0.0312 & -0.0331 \\
\hline SSD & 0.0561 & 0.0658 & 0.0751 & 0.0586 & 0.0286 & 0.0229 & 0.0240 & 0.0253 \\
\hline \multirow[t]{2}{*}{ SE } & 0.0099 & 0.0116 & 0.0133 & 0.0104 & 0.0051 & 0.0041 & 0.0043 & 0.0045 \\
\hline & \multicolumn{4}{|c|}{$E g(y)$} & \multicolumn{4}{|c|}{$A 1 g$} \\
\hline MEAN & 0.0686 & 0.0671 & 0.0645 & 0.1488 & 0.2576 & 0.2679 & 0.2499 & 0.2006 \\
\hline SSD & 0.0602 & 0.0515 & 0.0620 & 0.0557 & 0.0558 & 0.0518 & 0.0352 & 0.0434 \\
\hline \multirow[t]{2}{*}{ SE } & 0.0106 & 0.0091 & 0.0110 & 0.0098 & 0.0099 & 0.0092 & 0.0062 & 0.0077 \\
\hline & \multicolumn{4}{|c|}{$A 1 u$} & \multicolumn{4}{|c|}{$A 2 g$} \\
\hline MEAN & 0.0706 & 0.0113 & -0.1827 & -0.2380 & -0.0777 & -0.0788 & -0.0866 & -0.0752 \\
\hline SSD & 0.0244 & 0.0229 & 0.0348 & 0.0435 & 0.0160 & 0.0187 & 0.0143 & 0.0180 \\
\hline SE & 0.0043 & 0.0041 & 0.0061 & 0.0077 & 0.0028 & 0.0033 & 0.0025 & 0.0032 \\
\hline
\end{tabular}

Table 19: Descriptive statistics of extended basis out-of-plane next-to-lowest energy normal-coordinate displacements for each ETC cofactor in all BBC1 experiments; $\mathrm{n}=13$.

\begin{tabular}{|c|c|c|c|c|c|c|c|c|}
\hline & BA & BB & $\mathrm{DL}$ & $\mathrm{DM}$ & BA & BB & $\mathrm{DL}$ & $\mathrm{DM}$ \\
\hline & \multicolumn{4}{|c|}{$B 2 u$} & \multicolumn{4}{|c|}{$B 2 g$} \\
\hline MEAN & 0.0670 & 0.0354 & 0.0390 & 0.0236 & 0.0210 & 0.0493 & 0.0062 & 0.0131 \\
\hline SSD & 0.0705 & 0.0287 & 0.0539 & 0.0393 & 0.0400 & 0.0507 & 0.0423 & 0.0332 \\
\hline \multirow[t]{2}{*}{ SE } & 0.0125 & 0.0051 & 0.0095 & 0.0069 & 0.0071 & 0.0090 & 0.0075 & 0.0059 \\
\hline & \multicolumn{4}{|c|}{$B 1 u$} & \multicolumn{4}{|c|}{ B1g } \\
\hline MEAN & -0.0514 & -0.0386 & 0.1249 & 0.1483 & -0.0720 & -0.0721 & -0.0789 & -0.0634 \\
\hline SSD & 0.0266 & 0.0426 & 0.0324 & 0.0302 & 0.0608 & 0.0543 & 0.0515 & 0.0549 \\
\hline SE & 0.0047 & 0.0075 & 0.0057 & 0.0053 & 0.0108 & 0.0096 & 0.0091 & 0.0097 \\
\hline
\end{tabular}




\begin{tabular}{|c|c|c|c|c|c|c|c|c|}
\hline & $B A$ & BB & $\mathrm{DL}$ & $\mathrm{DM}$ & BA & BB & $\mathrm{DL}$ & $\mathrm{DM}$ \\
\hline & \multicolumn{4}{|c|}{$A 2 u$} & \multicolumn{4}{|c|}{$E u(x)$} \\
\hline MEAN & -0.0752 & -0.0243 & 0.0056 & 0.0056 & -0.0541 & -0.0424 & -0.0580 & -0.0425 \\
\hline SSD & 0.0657 & 0.0563 & 0.0435 & 0.0442 & 0.0265 & 0.0282 & 0.0280 & 0.0327 \\
\hline \multirow[t]{2}{*}{ SE } & 0.0116 & 0.0100 & 0.0077 & 0.0078 & 0.0047 & 0.0050 & 0.0050 & 0.0058 \\
\hline & \multicolumn{4}{|c|}{$E g(x)$} & \multicolumn{4}{|c|}{$E u(y)$} \\
\hline MEAN & 0.0955 & 0.1926 & -0.0660 & -0.0347 & 0.1171 & 0.1132 & 0.1282 & 0.1320 \\
\hline SSD & 0.0424 & 0.0964 & 0.0425 & 0.0593 & 0.0208 & 0.0320 & 0.0141 & 0.0203 \\
\hline \multirow[t]{2}{*}{ SE } & 0.0075 & 0.0170 & 0.0075 & 0.0105 & 0.0037 & 0.0057 & 0.0025 & 0.0036 \\
\hline & \multicolumn{4}{|c|}{$E g(y)$} & \multicolumn{4}{|c|}{ A1g } \\
\hline MEAN & 0.0303 & 0.0062 & -0.0135 & 0.0184 & -0.0038 & -0.0027 & 0.0208 & 0.0485 \\
\hline SSD & 0.0581 & 0.0603 & 0.0705 & 0.0615 & 0.0415 & 0.0378 & 0.0465 & 0.0519 \\
\hline \multirow[t]{2}{*}{ SE } & 0.0103 & 0.0107 & 0.0125 & 0.0109 & 0.0073 & 0.0067 & 0.0082 & 0.0092 \\
\hline & \multicolumn{4}{|c|}{ A1u } & \multicolumn{4}{|c|}{$A 2 g$} \\
\hline MEAN & -0.0201 & 0.0018 & 0.0948 & 0.1111 & -0.0003 & 0.0029 & 0.0170 & 0.0080 \\
\hline SSD & 0.0159 & 0.0187 & 0.0309 & 0.0449 & 0.0323 & 0.0317 & 0.0335 & 0.0326 \\
\hline SE & 0.0028 & 0.0033 & 0.0055 & 0.0079 & 0.0057 & 0.0056 & 0.0059 & 0.0058 \\
\hline
\end{tabular}

5.7.6 Rhodobacter sphaeroides bacteriopheophytins - RBP2

Table 20: Descriptive statistics of minimum basis in- and out-of-plane parameters and normal-coordinate displacements for each ETC cofactor in all RBP2 experiments; $\mathrm{n}=16$.

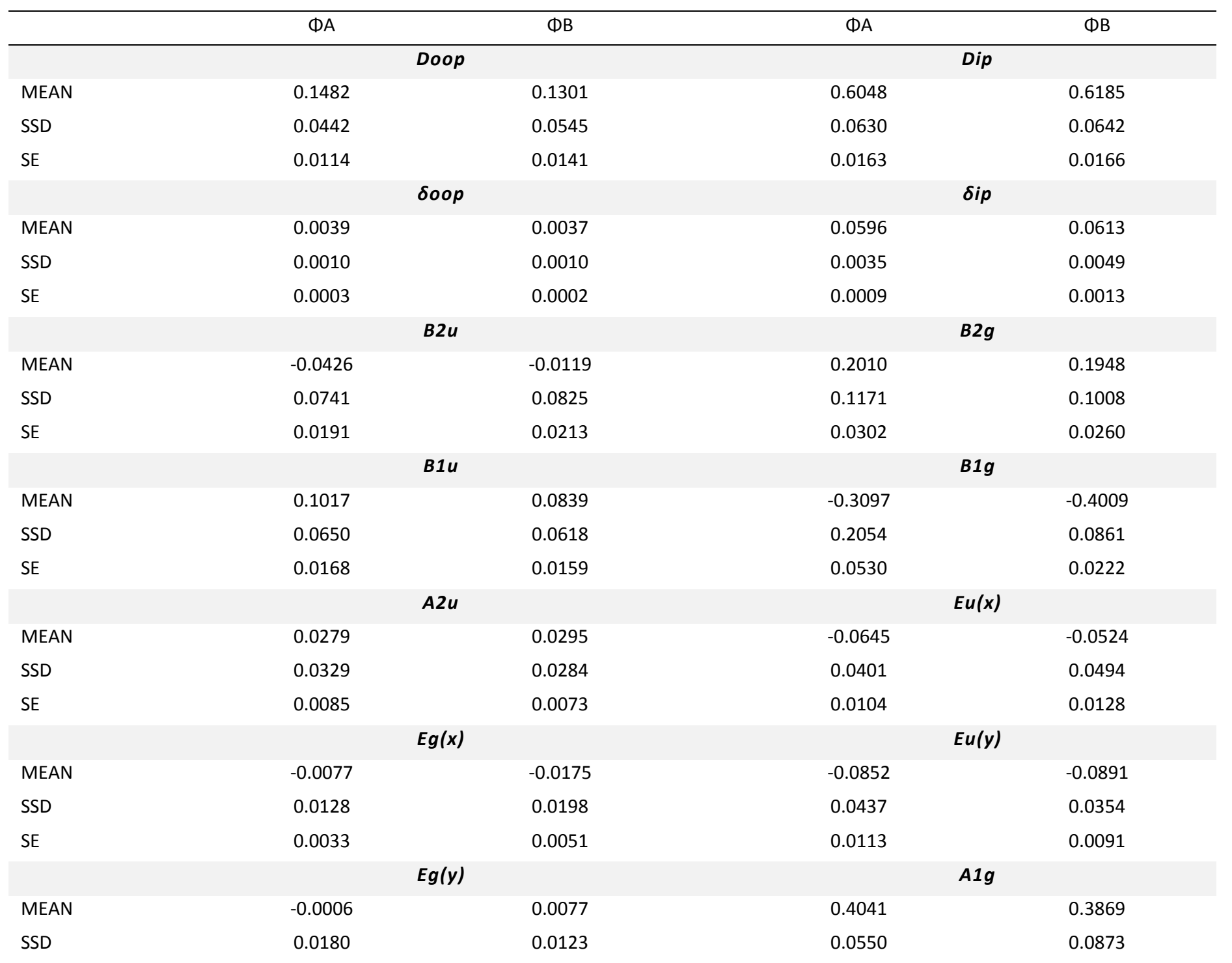




\begin{tabular}{|c|c|c|c|c|}
\hline & $\Phi A$ & ФB & $\Phi A$ & ФB \\
\hline \multirow[t]{2}{*}{ SE } & 0.0046 & 0.0032 & 0.0142 & 0.0225 \\
\hline & \multicolumn{2}{|c|}{ A1u } & \multicolumn{2}{|c|}{$A 2 g$} \\
\hline MEAN & 0.0151 & 0.0110 & -0.0115 & -0.0200 \\
\hline SSD & 0.0065 & 0.0040 & 0.0108 & 0.0044 \\
\hline SE & 0.0017 & 0.0010 & 0.0028 & 0.0011 \\
\hline
\end{tabular}

5.7.7 Rhodobacter sphaeroides bacteriopheophytins - RBP3

Table 21: Descriptive statistics of minimum basis in- and out-of-plane parameters and normal-coordinate displacements for each ETC cofactor in all RBP3 experiments; $\mathrm{n}=17$.

\begin{tabular}{|c|c|c|c|c|}
\hline & $\Phi A$ & ФB & $\Phi A$ & ФB \\
\hline & \multicolumn{2}{|c|}{ Doop } & \multicolumn{2}{|c|}{ Dip } \\
\hline MEAN & 0.6118 & 0.6362 & 0.6870 & 0.7161 \\
\hline SSD & 0.0638 & 0.1280 & 0.0670 & 0.0488 \\
\hline \multirow[t]{2}{*}{ SE } & 0.0213 & 0.0427 & 0.0223 & 0.0163 \\
\hline & \multicolumn{2}{|c|}{  } & \multicolumn{2}{|c|}{ sip } \\
\hline MEAN & 0.0648 & 0.0768 & 0.0605 & 0.0653 \\
\hline SSD & 0.0178 & 0.0254 & 0.0049 & 0.0073 \\
\hline \multirow[t]{2}{*}{ SE } & 0.0059 & 0.0085 & 0.0016 & 0.0024 \\
\hline & \multicolumn{2}{|c|}{$B 2 u$} & \multicolumn{2}{|c|}{$B 2 g$} \\
\hline MEAN & 0.0365 & 0.2596 & 0.3392 & 0.3309 \\
\hline SSD & 0.0722 & 0.1128 & 0.0883 & 0.0581 \\
\hline \multirow[t]{2}{*}{ SE } & 0.0241 & 0.0376 & 0.0294 & 0.0194 \\
\hline & \multicolumn{2}{|c|}{$B 1 u$} & \multicolumn{2}{|c|}{ B1g } \\
\hline MEAN & 0.4511 & 0.4048 & -0.4287 & -0.4732 \\
\hline SSD & 0.1022 & 0.1135 & 0.0809 & 0.0986 \\
\hline \multirow[t]{2}{*}{ SE } & 0.0341 & 0.0378 & 0.0270 & 0.0329 \\
\hline & \multicolumn{2}{|c|}{$A 2 u$} & \multicolumn{2}{|c|}{$E u(x)$} \\
\hline MEAN & 0.2451 & 0.2729 & -0.1415 & -0.0723 \\
\hline SSD & 0.0802 & 0.1109 & 0.0387 & 0.0509 \\
\hline \multirow[t]{2}{*}{ SE } & 0.0267 & 0.0370 & 0.0129 & 0.0170 \\
\hline & \multicolumn{2}{|c|}{$E g(x)$} & \multicolumn{2}{|c|}{$E u(y)$} \\
\hline MEAN & -0.0564 & -0.1562 & -0.0955 & -0.1070 \\
\hline SSD & 0.0742 & 0.1017 & 0.0358 & 0.0571 \\
\hline \multirow[t]{2}{*}{ SE } & 0.0247 & 0.0339 & 0.0119 & 0.0190 \\
\hline & \multicolumn{2}{|c|}{$E g(y)$} & \multicolumn{2}{|c|}{ A1g } \\
\hline MEAN & -0.0355 & 0.0019 & 0.3354 & 0.3609 \\
\hline SSD & 0.0798 & 0.1304 & 0.0850 & 0.0895 \\
\hline \multirow[t]{2}{*}{ SE } & 0.0266 & 0.0435 & 0.0283 & 0.0298 \\
\hline & \multicolumn{2}{|c|}{$A 1 u$} & \multicolumn{2}{|c|}{$A 2 g$} \\
\hline MEAN & 0.2711 & 0.1321 & -0.1118 & -0.0934 \\
\hline SSD & 0.0590 & 0.1090 & 0.0191 & 0.0250 \\
\hline SE & 0.0197 & 0.0363 & 0.0064 & 0.0083 \\
\hline
\end{tabular}


5.7.8 Rhodobacter sphaeroides bacteriopheophytins - RBP4

Table 22: Descriptive statistics of minimum basis in- and out-of-plane parameters and normal-coordinate displacements for each ETC cofactor in all RBP4 experiments; $\mathbf{n}=16$.

\begin{tabular}{|c|c|c|c|c|}
\hline & $\Phi A$ & ФB & $\Phi A$ & DB \\
\hline & \multicolumn{2}{|c|}{ Doop } & \multicolumn{2}{|c|}{ Dip } \\
\hline MEAN & 0.5585 & 0.5080 & 0.5779 & 0.6320 \\
\hline SSD & 0.0514 & 0.0779 & 0.0498 & 0.0818 \\
\hline \multirow[t]{2}{*}{ SE } & 0.0171 & 0.0260 & 0.0166 & 0.0273 \\
\hline & \multicolumn{2}{|c|}{ Soop } & \multicolumn{2}{|c|}{$\delta i p$} \\
\hline MEAN & 0.0260 & 0.0232 & 0.0553 & 0.0561 \\
\hline SSD & 0.0074 & 0.0060 & 0.0068 & 0.0046 \\
\hline \multirow[t]{2}{*}{ SE } & 0.0025 & 0.0020 & 0.0023 & 0.0015 \\
\hline & \multicolumn{2}{|c|}{$B 2 u$} & \multicolumn{2}{|c|}{$B 2 g$} \\
\hline MEAN & 0.0200 & 0.1073 & 0.1757 & 0.1929 \\
\hline SSD & 0.0766 & 0.0633 & 0.1255 & 0.1563 \\
\hline \multirow[t]{2}{*}{ SE } & 0.0255 & 0.0211 & 0.0418 & 0.0521 \\
\hline & \multicolumn{2}{|c|}{ B1u } & \multicolumn{2}{|c|}{ B1g } \\
\hline MEAN & 0.4724 & 0.4156 & -0.3540 & -0.3919 \\
\hline SSD & 0.0659 & 0.0821 & 0.0731 & 0.0851 \\
\hline \multirow[t]{2}{*}{ SE } & 0.0220 & 0.0274 & 0.0244 & 0.0284 \\
\hline & \multicolumn{2}{|c|}{$A 2 u$} & \multicolumn{2}{|c|}{$E u(x)$} \\
\hline MEAN & 0.2084 & 0.1835 & -0.0855 & -0.0360 \\
\hline SSD & 0.0609 & 0.0567 & 0.0348 & 0.0535 \\
\hline \multirow[t]{2}{*}{ SE } & 0.0203 & 0.0189 & 0.0116 & 0.0178 \\
\hline & \multicolumn{2}{|c|}{$E g(x)$} & \multicolumn{2}{|c|}{$E u(y)$} \\
\hline MEAN & -0.0677 & -0.1116 & -0.1022 & -0.1241 \\
\hline SSD & 0.0862 & 0.0525 & 0.0433 & 0.0438 \\
\hline \multirow[t]{2}{*}{ SE } & 0.0287 & 0.0175 & 0.0144 & 0.0146 \\
\hline & \multicolumn{2}{|c|}{$E g(y)$} & \multicolumn{2}{|c|}{$A 1 g$} \\
\hline MEAN & -0.0004 & 0.0373 & 0.3586 & 0.3909 \\
\hline SSD & 0.0511 & 0.0894 & 0.0520 & 0.0601 \\
\hline \multirow[t]{2}{*}{ SE } & 0.0170 & 0.0298 & & \\
\hline & \multicolumn{2}{|c|}{$A 1 u$} & \multicolumn{2}{|c|}{$A 2 g$} \\
\hline MEAN & 0.1394 & 0.0912 & -0.0898 & -0.0839 \\
\hline SSD & 0.0301 & 0.0241 & 0.0230 & 0.0286 \\
\hline SE & 0.0100 & 0.0080 & 0.0077 & 0.0095 \\
\hline
\end{tabular}

\subsubsection{Rhodobacter sphaeroides bacteriopheophytins - RBP5}

Table 23: Descriptive statistics of minimum basis in- and out-of-plane parameters and normal-coordinate displacements for each ETC cofactor in all RBP5 experiments; $\mathrm{n}=9$.

\begin{tabular}{|c|c|c|c|c|}
\hline & $\Phi A$ & ФB & ФA & DB \\
\hline & \multicolumn{2}{|c|}{ Doop } & \multicolumn{2}{|c|}{ Dip } \\
\hline MEAN & 0.4511 & 0.4571 & 0.3801 & 0.3615 \\
\hline SSD & 0.1181 & 0.0972 & 0.0681 & 0.0323 \\
\hline \multirow[t]{2}{*}{ SE } & 0.0394 & 0.0324 & 0.0227 & 0.0108 \\
\hline & \multicolumn{2}{|c|}{ Soop } & \multicolumn{2}{|c|}{ sip } \\
\hline MEAN & 0.0169 & 0.0159 & 0.0549 & 0.0541 \\
\hline SSD & 0.0069 & 0.0076 & 0.0041 & 0.0016 \\
\hline SE & 0.0023 & 0.0025 & 0.0014 & 0.0005 \\
\hline
\end{tabular}




\begin{tabular}{|c|c|c|c|c|}
\hline & $\Phi A$ & ФB & $\Phi A$ & ФB \\
\hline & \multicolumn{2}{|c|}{$B 2 u$} & \multicolumn{2}{|c|}{$B 2 g$} \\
\hline MEAN & 0.0265 & 0.0769 & -0.0469 & -0.0557 \\
\hline SSD & 0.1259 & 0.0397 & 0.0657 & 0.1468 \\
\hline \multirow[t]{2}{*}{ SE } & 0.0420 & 0.0132 & 0.0219 & 0.0489 \\
\hline & \multicolumn{2}{|c|}{ B1u } & \multicolumn{2}{|c|}{ B1g } \\
\hline MEAN & 0.3857 & 0.3992 & -0.2718 & -0.2325 \\
\hline SSD & 0.1314 & 0.1119 & 0.0769 & 0.0526 \\
\hline \multirow[t]{2}{*}{ SE } & 0.0438 & 0.0373 & 0.0256 & 0.0175 \\
\hline & \multicolumn{2}{|c|}{$A 2 u$} & \multicolumn{2}{|c|}{$E u(x)$} \\
\hline MEAN & 0.0981 & 0.1564 & -0.0748 & -0.0376 \\
\hline SSD & 0.1047 & 0.0655 & 0.0391 & 0.0521 \\
\hline \multirow[t]{2}{*}{ SE } & 0.0349 & 0.0218 & 0.0130 & 0.0174 \\
\hline & \multicolumn{2}{|c|}{$E g(x)$} & \multicolumn{2}{|c|}{$E u(y)$} \\
\hline MEAN & -0.0111 & -0.0329 & -0.0409 & -0.0483 \\
\hline SSD & 0.0706 & 0.0592 & 0.0425 & 0.0417 \\
\hline \multirow[t]{2}{*}{ SE } & 0.0235 & 0.0197 & 0.0142 & 0.0139 \\
\hline & \multicolumn{2}{|c|}{$E g(y)$} & \multicolumn{2}{|c|}{ A1g } \\
\hline MEAN & -0.0560 & 0.0141 & 0.2143 & 0.1965 \\
\hline SSD & 0.0877 & 0.0607 & 0.0711 & 0.0432 \\
\hline \multirow[t]{2}{*}{ SE } & 0.0292 & 0.0202 & 0.0237 & 0.0144 \\
\hline & \multicolumn{2}{|c|}{$A 1 u$} & \multicolumn{2}{|c|}{$A 2 g$} \\
\hline MEAN & 0.0460 & 0.0415 & -0.0462 & -0.0679 \\
\hline SSD & 0.0333 & 0.0460 & 0.0266 & 0.0160 \\
\hline SE & 0.0111 & 0.0153 & 0.0089 & 0.0053 \\
\hline
\end{tabular}

\subsubsection{Blastochloris viridis bacteriopheophytins - BBP1}

Table 24: Descriptive statistics of minimum basis in- and out-of-plane parameters and normal-coordinate displacements for each ETC cofactor in all BBP1 experiments; $n=13$.

\begin{tabular}{|c|c|c|c|c|}
\hline & $\Phi A$ & $\Phi B$ & $\Phi A$ & ФB \\
\hline \multicolumn{3}{|c|}{ Doop } & \multicolumn{2}{|c|}{ Dip } \\
\hline MEAN & 0.5271 & 0.6099 & 0.5529 & 0.5665 \\
\hline SSD & 0.0650 & 0.1122 & 0.0984 & 0.0417 \\
\hline \multirow[t]{2}{*}{ SE } & 0.0180 & 0.0311 & 0.0273 & 0.0116 \\
\hline & \multicolumn{2}{|c|}{ Soop } & \multicolumn{2}{|c|}{ sip } \\
\hline MEAN & 0.0301 & 0.0378 & 0.0493 & 0.0485 \\
\hline SSD & 0.0066 & 0.0093 & 0.0081 & 0.0101 \\
\hline \multirow[t]{2}{*}{ SE } & 0.0018 & 0.0026 & 0.0022 & 0.0028 \\
\hline & \multicolumn{2}{|c|}{$B 2 u$} & \multicolumn{2}{|c|}{$B 2 g$} \\
\hline MEAN & -0.2420 & -0.1369 & 0.3464 & 0.2090 \\
\hline SSD & 0.1193 & 0.1210 & 0.0923 & 0.0882 \\
\hline \multirow[t]{2}{*}{ SE } & 0.0331 & 0.0336 & 0.0256 & 0.0245 \\
\hline & \multicolumn{2}{|c|}{$B 1 u$} & \multicolumn{2}{|c|}{ B1g } \\
\hline MEAN & 0.3812 & 0.5148 & -0.2115 & -0.3877 \\
\hline SSD & 0.0540 & 0.1144 & 0.1323 & 0.0713 \\
\hline \multirow[t]{2}{*}{ SE } & 0.0150 & 0.0317 & 0.0367 & 0.0198 \\
\hline & \multicolumn{2}{|c|}{$A 2 u$} & \multicolumn{2}{|c|}{$E u(x)$} \\
\hline MEAN & 0.0571 & 0.0380 & -0.1132 & -0.0869 \\
\hline SSD & 0.0617 & 0.0756 & 0.0374 & 0.0479 \\
\hline
\end{tabular}




\begin{tabular}{|c|c|c|c|c|}
\hline & DA & ФB & $\Phi A$ & ФB \\
\hline \multirow[t]{2}{*}{ SE } & 0.0171 & 0.0210 & 0.0104 & 0.0133 \\
\hline & \multicolumn{2}{|c|}{$E g(x)$} & \multicolumn{2}{|c|}{$E u(y)$} \\
\hline MEAN & -0.1818 & -0.1591 & -0.0338 & -0.0265 \\
\hline SSD & 0.0709 & 0.0603 & 0.0340 & 0.0268 \\
\hline \multirow[t]{2}{*}{ SE } & 0.0197 & 0.0167 & 0.0094 & 0.0074 \\
\hline & \multicolumn{2}{|c|}{$E g(y)$} & \multicolumn{2}{|c|}{$A 1 g$} \\
\hline MEAN & -0.0243 & -0.0140 & 0.3136 & 0.3117 \\
\hline SSD & 0.0616 & 0.1088 & 0.0627 & 0.0421 \\
\hline \multirow[t]{2}{*}{ SE } & 0.0171 & 0.0302 & 0.0174 & 0.0117 \\
\hline & \multicolumn{2}{|c|}{ A1u } & \multicolumn{2}{|c|}{$A 2 g$} \\
\hline MEAN & 0.1082 & 0.1547 & -0.0838 & -0.0801 \\
\hline SSD & 0.0435 & 0.0648 & 0.0264 & 0.0217 \\
\hline SE & 0.0121 & 0.0180 & 0.0073 & 0.0060 \\
\hline
\end{tabular}

\subsection{Tabular re-centred NSD deformations and parameters}

5.8.1 Rhodobacter sphaeroides bacteriochlorophylls - RBC4

Table 25: Minimum basis parameters and normal-coordinate displacements of each ETC cofactor in RBC4 (average coordinates).

\begin{tabular}{|c|c|c|c|c|c|c|c|}
\hline$B A$ & BB & $\mathrm{DL}$ & $\mathrm{DM}$ & $B A$ & BB & $\mathrm{DL}$ & $\mathrm{DM}$ \\
\hline \multicolumn{4}{|c|}{ Doop } & \multicolumn{4}{|c|}{ Dip } \\
\hline 0.3198 & 0.5623 & 0.4905 & 0.5447 & 0.2704 & 0.3662 & 0.3792 & 0.3501 \\
\hline \multicolumn{4}{|c|}{$\delta o o p$} & \multicolumn{4}{|c|}{ sip } \\
\hline 0.0268 & 0.0170 & 0.0267 & 0.0110 & 0.0445 & 0.0463 & 0.0445 & 0.0488 \\
\hline \multicolumn{4}{|c|}{$B 2 u$} & \multicolumn{4}{|c|}{$B 2 g$} \\
\hline 0.0688 & 0.1937 & 0.2455 & 0.2625 & 0.0361 & 0.0340 & -0.0261 & 0.0022 \\
\hline \multicolumn{4}{|c|}{ B1u } & \multicolumn{4}{|c|}{ B1g } \\
\hline 0.0557 & 0.4215 & 0.1834 & -0.4498 & -0.1221 & -0.2316 & -0.2238 & -0.2020 \\
\hline \multicolumn{4}{|c|}{$A 2 u$} & \multicolumn{4}{|c|}{$E u(x)$} \\
\hline-0.1467 & -0.0658 & -0.3326 & -0.1008 & -0.0718 & -0.0465 & -0.0786 & -0.0558 \\
\hline \multicolumn{4}{|c|}{$E g(x)$} & \multicolumn{4}{|c|}{$E u(y)$} \\
\hline-0.2700 & -0.2763 & -0.0953 & -0.1004 & -0.0323 & -0.1038 & -0.0680 & -0.0337 \\
\hline \multicolumn{4}{|c|}{$E g(y)$} & \multicolumn{4}{|c|}{$A 1 g$} \\
\hline 0.0004 & 0.1352 & -0.1623 & -0.0229 & 0.2120 & 0.2471 & 0.2753 & 0.2700 \\
\hline \multicolumn{4}{|c|}{$A 1 u$} & \multicolumn{4}{|c|}{$A 2 g$} \\
\hline-0.0025 & 0.0452 & 0.0256 & -0.0688 & -0.0762 & -0.0728 & -0.0799 & -0.0677 \\
\hline
\end{tabular}

\subsubsection{Blastochloris viridis bacteriochlorophylls - BBC1}

\section{Minimum Basis}

Table 26: Minimum basis parameters and normal-coordinate displacements of each ETC cofactor in BBC1 (from averaged coordinates).

\begin{tabular}{|c|c|c|c|c|c|c|c|}
\hline $\mathrm{BA}$ & BB & $\mathrm{DL}$ & $\mathrm{DM}$ & BA & BB & $\mathrm{DL}$ & DM \\
\hline \multicolumn{4}{|c|}{ Doop } & \multicolumn{4}{|c|}{ Dip } \\
\hline 0.4262 & 0.4024 & 0.7467 & 1.0031 & 0.4066 & 0.3884 & 0.3682 & 0.3088 \\
\hline \multicolumn{4}{|c|}{ Soop } & \multicolumn{4}{|c|}{ Sip } \\
\hline 0.0424 & 0.0443 & 0.0319 & 0.0365 & 0.0377 & 0.0367 & 0.0389 & 0.0347 \\
\hline \multicolumn{4}{|c|}{$B 2 u$} & \multicolumn{4}{|c|}{$B 2 g$} \\
\hline 0.1174 & 0.0226 & -0.2390 & -0.0769 & 0.0802 & 0.0549 & 0.0568 & 0.0481 \\
\hline
\end{tabular}




\begin{tabular}{|c|c|c|c|c|c|c|c|}
\hline \multicolumn{4}{|c|}{$B 1 u$} & \multicolumn{4}{|c|}{$B 1 g$} \\
\hline 0.123 & 0.0363 & -0.6791 & -0.9567 & -0.2642 & -0.2519 & -0.2148 & -0.1792 \\
\hline \multicolumn{4}{|c|}{$A 2 u$} & \multicolumn{4}{|c|}{$E u(x)$} \\
\hline-0.0517 & -0.1638 & -0.0366 & -0.0417 & -0.1098 & -0.0784 & -0.1237 & -0.1189 \\
\hline \multicolumn{4}{|c|}{$E g(x)$} & \multicolumn{4}{|c|}{$E u(y)$} \\
\hline-0.3750 & -0.3588 & 0.0071 & -0.0662 & -0.0701 & -0.0262 & -0.0323 & -0.0351 \\
\hline \multicolumn{4}{|c|}{$E g(y)$} & \multicolumn{4}{|c|}{$A 1 g$} \\
\hline 0.0671 & 0.0670 & 0.0654 & 0.1497 & 0.2569 & 0.2670 & 0.2495 & 0.1992 \\
\hline \multicolumn{4}{|c|}{$A 1 u$} & \multicolumn{4}{|c|}{$A 2 g$} \\
\hline 0.0704 & 0.0110 & -0.1833 & -0.2377 & -0.0783 & -0.0789 & -0.0875 & -0.0767 \\
\hline
\end{tabular}

\section{Extended Basis}

Table 27: Extended basis parameters and normal-coordinate displacements of each ETC cofactor in BBC1 (from averaged coordinates).

\begin{tabular}{|c|c|c|c|c|c|c|c|c|}
\hline & $B A$ & BB & $\mathrm{DL}$ & $\mathrm{DM}$ & BA & BB & $\mathrm{DL}$ & DM \\
\hline & \multicolumn{4}{|c|}{ Doop } & \multicolumn{4}{|c|}{ Dip } \\
\hline \multirow[t]{2}{*}{ N/A } & 0.4525 & 0.4505 & 0.7672 & 1.0212 & 0.4331 & 0.4158 & 0.4029 & 0.3481 \\
\hline & \multicolumn{4}{|c|}{ Soop } & \multicolumn{4}{|c|}{ Sip } \\
\hline \multirow[t]{2}{*}{ N/A } & 0.0341 & 0.034 & 0.0192 & 0.0158 & 0.0223 & 0.0229 & 0.0213 & 0.0179 \\
\hline & \multicolumn{4}{|c|}{$B 2 u$} & \multicolumn{4}{|c|}{$B 2 g$} \\
\hline $1^{\text {st }}$ & 0.1192 & 0.0235 & -0.2386 & -0.0767 & 0.0807 & 0.0558 & 0.0578 & 0.0488 \\
\hline \multirow[t]{2}{*}{$2^{\text {nd }}$} & 0.0672 & 0.0352 & 0.0391 & 0.0236 & 0.0208 & 0.0495 & 0.0057 & 0.0133 \\
\hline & \multicolumn{4}{|c|}{ B1u } & \multicolumn{4}{|c|}{ B1g } \\
\hline $1^{\text {st }}$ & 0.1230 & 0.0363 & -0.6791 & -0.9567 & -0.2645 & -0.2522 & -0.215 & -0.1796 \\
\hline \multirow[t]{2}{*}{$2^{\text {nd }}$} & -0.0510 & -0.0382 & 0.1247 & 0.1481 & -0.0722 & -0.0720 & -0.0787 & -0.0630 \\
\hline & \multicolumn{4}{|c|}{$A 2 u$} & \multicolumn{4}{|c|}{$E u(x)$} \\
\hline $1^{\text {st }}$ & -0.057 & -0.1666 & -0.0366 & -0.0421 & -0.1103 & -0.0790 & -0.1243 & -0.1194 \\
\hline \multirow[t]{2}{*}{$2^{\text {nd }}$} & -0.0752 & -0.0242 & 0.0055 & 0.006 & -0.0541 & -0.0426 & -0.0576 & -0.0429 \\
\hline & \multicolumn{4}{|c|}{$E g(x)$} & \multicolumn{4}{|c|}{$E u(y)$} \\
\hline $1^{\text {st }}$ & -0.3728 & -0.3526 & 0.0054 & -0.0671 & -0.0684 & -0.0247 & -0.0307 & -0.0335 \\
\hline \multirow[t]{2}{*}{$2^{\text {nd }}$} & 0.0951 & 0.1926 & -0.0663 & -0.0351 & 0.1173 & 0.1130 & 0.1288 & 0.1323 \\
\hline & \multicolumn{4}{|c|}{$E g(y)$} & \multicolumn{4}{|c|}{$A 1 g$} \\
\hline $1^{\text {st }}$ & 0.0681 & 0.067 & 0.0643 & 0.1488 & 0.2577 & 0.2679 & 0.25 & 0.2005 \\
\hline \multirow[t]{2}{*}{$2^{\text {nd }}$} & 0.0306 & 0.0064 & -0.0134 & 0.0187 & -0.0042 & -0.0024 & 0.0204 & 0.0479 \\
\hline & \multicolumn{4}{|c|}{ A1u } & \multicolumn{4}{|c|}{$A 2 g$} \\
\hline $1^{\text {st }}$ & 0.0704 & 0.011 & -0.1833 & -0.2377 & -0.0778 & -0.0785 & -0.0865 & -0.0757 \\
\hline $2^{\text {nd }}$ & -0.0200 & 0.0022 & 0.0949 & 0.1107 & -0.0005 & 0.0029 & 0.0169 & 0.0081 \\
\hline
\end{tabular}

\subsubsection{Rhodobacter sphaeroides bacteriopheophytins - RBP4}

Table 28: Minimum basis in- and out-of-plane parameters and normal-coordinate displacements for each ETC cofactor in all RBP4 experiments.

\begin{tabular}{|c|c|c|c|}
\hline$\Phi A$ & ФB & $\Phi A$ & ФB \\
\hline \multicolumn{2}{|c|}{ Doop } & \multicolumn{2}{|c|}{ Dip } \\
\hline 0.5394 & 0.4902 & 0.5573 & 0.6061 \\
\hline \multicolumn{2}{|c|}{$\delta o o p$} & \multicolumn{2}{|c|}{$\delta i p$} \\
\hline 0.0211 & 0.0184 & 0.0494 & 0.0518 \\
\hline \multicolumn{2}{|c|}{$B 2 u$} & \multicolumn{2}{|c|}{$B 2 g$} \\
\hline 0.0204 & 0.1073 & 0.1755 & 0.1923 \\
\hline
\end{tabular}


B1u

0.4724

$A 2 u$

0.2083

$-0.0673$

$E g(y)$

$-0.0004$

0.1395
$E g(x)$

g(y)

A1u

0.0372

0.0916
B1g

$-0.3539$

$-0.3921$

$E u(x)$

$-0.0851$

$-0.0358$

$E u(y)$

$-0.1020$

$-0.1245$

A1g

0.3590

0.3909

A2g

$-0.0897$

$-0.0836$

\subsubsection{Rhodobacter sphaeroides bacteriopheophytins - RBP5}

Table 29: Minimum basis in- and out-of-plane parameters and normal-coordinate displacements for each ETC cofactor in all RBP5 experiments.

\begin{tabular}{|c|c|c|c|}
\hline$\Phi A$ & ФB & $\Phi A$ & ФB \\
\hline \multicolumn{2}{|c|}{ Doop } & \multicolumn{2}{|c|}{ Dip } \\
\hline 0.4057 & 0.4391 & 0.3632 & 0.3226 \\
\hline \multicolumn{2}{|c|}{ Soop } & \multicolumn{2}{|c|}{ sip } \\
\hline 0.0101 & 0.0085 & 0.0527 & 0.0513 \\
\hline \multicolumn{2}{|c|}{$B 2 u$} & \multicolumn{2}{|c|}{$B 2 g$} \\
\hline 0.0263 & 0.0774 & -0.0475 & -0.0556 \\
\hline \multicolumn{2}{|c|}{ B1u } & \multicolumn{2}{|c|}{$B 1 g$} \\
\hline 0.386 & 0.3993 & -0.2727 & -0.2323 \\
\hline \multicolumn{2}{|c|}{$A 2 u$} & \multicolumn{2}{|c|}{$E u(x)$} \\
\hline 0.0978 & 0.1564 & -0.075 & -0.0382 \\
\hline \multicolumn{2}{|c|}{$E g(x)$} & \multicolumn{2}{|c|}{$E u(y)$} \\
\hline-0.0113 & -0.0326 & -0.0406 & -0.0479 \\
\hline \multicolumn{2}{|c|}{$E g(y)$} & \multicolumn{2}{|c|}{$A 1 g$} \\
\hline-0.056 & 0.0141 & 0.2143 & 0.1967 \\
\hline \multicolumn{2}{|c|}{ A1u } & \multicolumn{2}{|c|}{$A 2 g$} \\
\hline 0.0458 & 0.0409 & -0.0459 & -0.0679 \\
\hline
\end{tabular}

5.8.5 Blastochloris viridis bacteriopheophytins - BBP1

Table 30: Minimum basis in- and out-of-plane parameters and normal-coordinate displacements for each ETC cofactor in all BBP1 experiments.

\begin{tabular}{|c|c|c|c|}
\hline$\Phi A$ & ФB & $\Phi A$ & ФB \\
\hline \multicolumn{2}{|c|}{ Doop } & \multicolumn{2}{|c|}{ Dip } \\
\hline 0.5021 & 0.5783 & 0.5329 & 0.5529 \\
\hline \multicolumn{2}{|c|}{$\delta o o p$} & \multicolumn{2}{|c|}{$\delta i p$} \\
\hline 0.0218 & 0.0325 & 0.0425 & 0.0415 \\
\hline \multicolumn{2}{|c|}{$B 2 u$} & \multicolumn{2}{|c|}{$B 2 g$} \\
\hline-0.2419 & -0.1368 & 0.3464 & 0.2088 \\
\hline \multicolumn{2}{|c|}{ B1u } & \multicolumn{2}{|c|}{ B1g } \\
\hline 0.381 & 0.5147 & -0.2112 & -0.388 \\
\hline \multicolumn{2}{|c|}{$A 2 u$} & \multicolumn{2}{|c|}{$E u(x)$} \\
\hline 0.0571 & 0.0381 & -0.1131 & -0.0867 \\
\hline
\end{tabular}




\begin{tabular}{cccccc}
\hline & $E g(x)$ & & & $E u(y)$ & -0.0263 \\
-0.1815 & $E g(y)$ & -0.159 & -0.0343 & $\boldsymbol{A 1 g}$ & 0.3114 \\
-0.0245 & $\boldsymbol{A 1 u}$ & -0.0137 & 0.3137 & $\boldsymbol{A 2 g}$ & -0.08 \\
\hline
\end{tabular}

\subsection{Cofactor Coordinates}

\subsubsection{Rhodobacter sphaeroides bacteriochlorophylls - RBC4}

Table 31: Atomic coordinates of the best estimate ( $R B C 4$ ) of the conformation of the $R$. sphaeroides BA macrocycle (angstroms). $\mathrm{n}=32$.

\begin{tabular}{|c|c|c|c|c|c|c|c|c|c|}
\hline \multirow[b]{2}{*}{ Atom } & \multicolumn{3}{|c|}{ Minimum } & \multicolumn{3}{|c|}{ Extended } & \multicolumn{3}{|c|}{ Complete } \\
\hline & $x$ & $y$ & $z$ & $x$ & y & z & $x$ & y & $z$ \\
\hline N1 & -2.0330 & -0.0311 & -0.1061 & -2.0343 & 0.0010 & -0.0364 & -2.0640 & 0.0060 & -0.0550 \\
\hline N2 & -0.0114 & 2.0712 & -0.0518 & 0.0173 & 2.0694 & -0.0922 & 0.0210 & 2.0591 & -0.0870 \\
\hline N3 & 2.0005 & 0.0297 & 0.0226 & 1.9808 & 0.0648 & 0.0100 & 2.0010 & 0.0630 & 0.0209 \\
\hline N4 & 0.0145 & -2.0566 & -0.0520 & 0.0389 & -2.0811 & -0.0675 & 0.0311 & -2.0689 & -0.0721 \\
\hline $\mathrm{Cm} 1$ & -2.4203 & 2.4363 & -0.0426 & -2.4322 & 2.4073 & -0.0540 & -2.4150 & 2.4080 & -0.0390 \\
\hline $\mathrm{Cm} 2$ & 2.4323 & 2.4467 & 0.0382 & 2.4712 & 2.4492 & 0.0570 & 2.4500 & 2.4411 & 0.0339 \\
\hline $\mathrm{Cm} 3$ & 2.4201 & -2.4268 & 0.0774 & 2.4181 & -2.3973 & 0.0866 & 2.4431 & -2.3819 & 0.0650 \\
\hline $\mathrm{Cm} 4$ & -2.4220 & -2.4607 & -0.0821 & -2.3987 & -2.4028 & -0.1020 & -2.4069 & -2.4080 & -0.0790 \\
\hline Ca1 & -2.8697 & -1.1304 & -0.0406 & -2.8580 & -1.0843 & -0.0143 & -2.8569 & -1.1040 & -0.0100 \\
\hline $\mathrm{Ca} 2$ & -2.8410 & 1.0898 & -0.0160 & -2.8698 & 1.0820 & 0.0158 & -2.8770 & 1.1050 & 0.0290 \\
\hline $\mathrm{Ca} 3$ & -1.0982 & 2.9136 & -0.0658 & -1.1022 & 2.8813 & -0.0976 & -1.0910 & 2.8481 & -0.0780 \\
\hline $\mathrm{Ca} 4$ & 1.1050 & 2.8852 & 0.0204 & 1.1346 & 2.8982 & 0.0165 & 1.1410 & 2.8662 & -0.0111 \\
\hline $\mathrm{Ca} 5$ & 2.8500 & 1.1140 & 0.0092 & 2.8683 & 1.1134 & 0.0127 & 2.8810 & 1.1152 & 0.0129 \\
\hline Ca6 & 2.8112 & -1.0866 & 0.0334 & 2.7735 & -1.0541 & 0.0228 & 2.7661 & -1.0438 & 0.0229 \\
\hline $\mathrm{Ca} 7$ & 1.1085 & -2.8912 & 0.0423 & 1.1269 & -2.9201 & 0.0529 & 1.1471 & -2.8930 & 0.0379 \\
\hline Ca8 & -1.0859 & -2.8853 & -0.0879 & -1.0744 & -2.8832 & -0.1144 & -1.0869 & -2.8500 & -0.0910 \\
\hline $\mathrm{Cb} 1$ & -4.2498 & -0.6850 & 0.1380 & -4.2539 & -0.6820 & 0.1170 & -4.2269 & -0.6970 & 0.1080 \\
\hline $\mathrm{Cb} 2$ & -4.2267 & 0.6769 & 0.1548 & -4.2597 & 0.6713 & 0.1471 & -4.2470 & 0.6790 & 0.1410 \\
\hline $\mathrm{Cb} 3$ & -0.6441 & 4.3007 & -0.0160 & -0.6724 & 4.2724 & -0.0208 & -0.7361 & 4.3121 & -0.0850 \\
\hline $\mathrm{Cb} 4$ & 0.7168 & 4.2755 & 0.0470 & 0.6958 & 4.2812 & 0.0737 & 0.7429 & 4.3021 & 0.1420 \\
\hline Cb5 & 4.2241 & 0.6599 & -0.0353 & 4.2357 & 0.6287 & -0.0362 & 4.2180 & 0.6352 & -0.0271 \\
\hline $\mathrm{Cb} 6$ & 4.1931 & -0.6963 & -0.0187 & 4.1627 & -0.7184 & -0.0232 & 4.1471 & -0.7388 & -0.0221 \\
\hline $\mathrm{Cb} 7$ & 0.6923 & -4.2786 & 0.0614 & 0.6989 & -4.3134 & 0.0809 & 0.7542 & -4.3459 & 0.1429 \\
\hline $\mathrm{Cb} 8$ & -0.6661 & -4.2709 & -0.0299 & -0.6672 & -4.2836 & -0.0345 & -0.7358 & -4.3089 & -0.1000 \\
\hline
\end{tabular}

Table 32: Atomic coordinates of the best estimate ( $R B C 4)$ of the conformation of the $R$. sphaeroides BB macrocycle (angstroms). $\mathrm{n}=32$.

\begin{tabular}{lccccccccc}
\hline & \multicolumn{3}{c}{ Minimum } & \multicolumn{3}{c}{ Extended } & \multicolumn{3}{c}{ Complete } \\
Atom & $\mathrm{x}$ & $\mathrm{y}$ & $\mathrm{z}$ & $\mathrm{x}$ & $\mathrm{y}$ & $\mathrm{z}$ & $\mathrm{x}$ & $\mathrm{y}$ \\
\hline $\mathrm{N} 1$ & -2.0131 & -0.0312 & -0.0726 & -2.0167 & 0.0037 & -0.0515 & -2.0511 & 0.0151 & -0.0651 \\
$\mathrm{~N} 2$ & -0.0115 & 2.1156 & -0.0030 & 0.0191 & 2.1124 & -0.0180 & 0.0171 & 2.0960 & -0.0150 \\
N3 & 1.9921 & 0.0267 & 0.0591 & 1.9732 & 0.0622 & 0.0416 & 1.9960 & 0.0629 & 0.0540 \\
N4 & 0.0135 & -2.0685 & -0.0675 & 0.0395 & -2.0930 & -0.0657 & 0.0349 & -2.0819 & -0.0771 \\
Cm1 & -2.4221 & 2.4442 & 0.1166 & -2.4265 & 2.4145 & 0.1126 & -2.4060 & 2.4141 & 0.1299 \\
Cm2 & 2.4318 & 2.4575 & -0.0584 & 2.4681 & 2.4566 & -0.0397 & 2.4501 & 2.4449 & -0.0530 \\
Cm3 & 2.4084 & -2.4438 & 0.1793 & 2.3987 & -2.4141 & 0.1834 & 2.4229 & -2.3981 & 0.1669 \\
\hline
\end{tabular}


Minimum

Extended

Complete

\begin{tabular}{lccccccccc} 
Atom & $\mathrm{x}$ & $\mathrm{y}$ & $\mathrm{z}$ & $\mathrm{x}$ & $\mathrm{y}$ & $\mathrm{z}$ & $\mathrm{x}$ & $\mathrm{y}$ \\
\hline Cm4 & -2.4116 & -2.4724 & -0.2415 & -2.3824 & -2.4077 & -0.2434 & -2.3921 & -2.4109 & -0.2401 \\
Ca1 & -2.8430 & -1.1398 & -0.1080 & -2.8328 & -1.0910 & -0.1046 & -2.8411 & -1.1139 & -0.0901 \\
Ca2 & -2.8271 & 1.0862 & 0.0997 & -2.8566 & 1.0788 & 0.1158 & -2.8610 & 1.1072 & 0.1159 \\
Ca3 & -1.1051 & 2.9532 & 0.0229 & -1.1035 & 2.9224 & 0.0087 & -1.0949 & 2.8901 & 0.0180 \\
Ca4 & 1.1069 & 2.9295 & -0.0640 & 1.1350 & 2.9399 & -0.0599 & 1.1401 & 2.9070 & -0.0760 \\
Ca5 & 2.8431 & 1.1122 & -0.0030 & 2.8639 & 1.1113 & -0.0082 & 2.8760 & 1.1120 & -0.0130 \\
Ca6 & 2.7949 & -1.1011 & 0.0962 & 2.7544 & -1.0680 & 0.0902 & 2.7519 & -1.0541 & 0.0850 \\
Ca7 & 1.1004 & -2.9102 & 0.1088 & 1.1145 & -2.9441 & 0.1168 & 1.1348 & -2.9130 & 0.1100 \\
Ca8 & -1.0832 & -2.9010 & -0.1996 & -1.0678 & -2.8951 & -0.2044 & -1.0812 & -2.8639 & -0.1711 \\
Cb1 & -4.2218 & -0.7098 & 0.1015 & -4.2298 & -0.7133 & 0.0917 & -4.2000 & -0.7288 & 0.0829 \\
Cb2 & -4.2087 & 0.6519 & 0.2338 & -4.2442 & 0.6402 & 0.2339 & -4.2230 & 0.6422 & 0.2319 \\
Cb3 & -0.6554 & 4.3478 & -0.0581 & -0.6839 & 4.3225 & -0.0573 & -0.7559 & 4.3541 & -0.1000 \\
Cb4 & 0.7096 & 4.3263 & -0.1191 & 0.6873 & 4.3309 & -0.1174 & 0.7452 & 4.3650 & -0.0730 \\
Cb5 & 4.2152 & 0.6364 & -0.0373 & 4.2306 & 0.6107 & -0.0363 & 4.2090 & 0.6179 & -0.0320 \\
Cb6 & 4.1768 & -0.7215 & 0.0178 & 4.1455 & -0.7386 & 0.0171 & 4.1279 & -0.7621 & 0.0230 \\
Cb7 & 0.6833 & -4.2992 & 0.1076 & 0.6888 & -4.3406 & 0.1196 & 0.7368 & -4.3770 & 0.1639 \\
Cb8 & -0.6734 & -4.2891 & -0.1111 & -0.6747 & -4.3007 & -0.1249 & -0.7362 & -4.3250 & -0.1761 \\
\hline & & & & & & & &
\end{tabular}

Table 33: Atomic coordinates of the best estimate (RBC4) of the conformation of the $R$. sphaeroides DL macrocycle (angstroms). $n=32$.

\begin{tabular}{|c|c|c|c|c|c|c|c|c|c|}
\hline \multirow[b]{2}{*}{ Atom } & \multicolumn{3}{|c|}{ Minimum } & \multicolumn{3}{|c|}{ Extended } & \multicolumn{3}{|c|}{ Complete } \\
\hline & $x$ & $y$ & $z$ & $x$ & $y$ & $z$ & $x$ & $y$ & $z$ \\
\hline N1 & -2.0275 & -0.0179 & -0.1108 & -2.0302 & 0.0160 & -0.0650 & -2.0610 & 0.0169 & -0.0801 \\
\hline N2 & -0.0274 & 2.1116 & -0.1629 & 0.0013 & 2.1080 & -0.1517 & 0.0010 & 2.0979 & -0.1541 \\
\hline N3 & 1.9919 & 0.0150 & -0.0654 & 1.9740 & 0.0499 & -0.1208 & 2.0030 & 0.0509 & -0.1171 \\
\hline N4 & 0.0307 & -2.0808 & -0.0856 & 0.0545 & -2.1041 & -0.0603 & 0.0430 & -2.0900 & -0.0661 \\
\hline $\mathrm{Cm} 1$ & -2.4338 & 2.4619 & 0.0024 & -2.4429 & 2.4381 & -0.0303 & -2.4301 & 2.4399 & -0.0201 \\
\hline $\mathrm{Cm} 2$ & 2.4209 & 2.4464 & -0.0849 & 2.4600 & 2.4499 & -0.0733 & 2.4399 & 2.4339 & -0.1081 \\
\hline $\mathrm{Cm} 3$ & 2.4282 & -2.4537 & 0.1170 & 2.4221 & -2.4285 & 0.1153 & 2.4430 & -2.4221 & 0.1040 \\
\hline $\mathrm{Cm} 4$ & -2.4042 & -2.4641 & -0.0550 & -2.3806 & -2.4066 & -0.0847 & -2.3821 & -2.4071 & -0.0672 \\
\hline Ca1 & -2.8527 & -1.1299 & -0.0035 & -2.8421 & -1.0852 & 0.0059 & -2.8461 & -1.1040 & 0.0108 \\
\hline $\mathrm{Ca} 2$ & -2.8426 & 1.1034 & -0.0047 & -2.8708 & 1.0990 & 0.0138 & -2.8780 & 1.1209 & 0.0239 \\
\hline $\mathrm{Ca} 3$ & -1.1130 & 2.9615 & -0.0537 & -1.1144 & 2.9319 & -0.0720 & -1.1061 & 2.8969 & -0.0551 \\
\hline $\mathrm{Ca} 4$ & 1.0977 & 2.9167 & -0.1044 & 1.1265 & 2.9260 & -0.0827 & 1.1330 & 2.8989 & -0.0880 \\
\hline $\mathrm{Ca} 5$ & 2.8362 & 1.1052 & -0.0511 & 2.8571 & 1.1068 & -0.0762 & 2.8760 & 1.1079 & -0.0561 \\
\hline Ca6 & 2.8050 & -1.1066 & 0.0778 & 2.7657 & -1.0766 & 0.0508 & 2.7600 & -1.0641 & 0.0440 \\
\hline $\mathrm{Ca} 7$ & 1.1198 & -2.9259 & 0.0144 & 1.1348 & -2.9574 & 0.0411 & 1.1530 & -2.9311 & 0.0240 \\
\hline Ca8 & -1.0723 & -2.9055 & -0.1128 & -1.0601 & -2.9006 & -0.1251 & -1.0711 & -2.8721 & -0.0981 \\
\hline Cb1 & -4.2356 & -0.6938 & 0.1990 & -4.2406 & -0.6971 & 0.1939 & -4.2110 & -0.7121 & 0.1829 \\
\hline $\mathrm{Cb} 2$ & -4.2250 & 0.6699 & 0.1904 & -4.2568 & 0.6591 & 0.1961 & -4.2410 & 0.6689 & 0.1948 \\
\hline $\mathrm{Cb} 3$ & -0.6470 & 4.3525 & 0.0772 & -0.6747 & 4.3263 & 0.0667 & -0.7471 & 4.3569 & 0.0169 \\
\hline $\mathrm{Cb} 4$ & 0.7174 & 4.3163 & 0.0405 & 0.6958 & 4.3183 & 0.0539 & 0.7579 & 4.3469 & 0.1039 \\
\hline Cb5 & 4.2114 & 0.6439 & 0.0914 & 4.2254 & 0.6182 & 0.1057 & 4.2019 & 0.6290 & 0.1070 \\
\hline $\mathrm{Cb} 6$ & 4.1842 & -0.7136 & 0.1755 & 4.1523 & -0.7313 & 0.1911 & 4.1290 & -0.7490 & 0.1950 \\
\hline $\mathrm{Cb} 7$ & 0.6984 & -4.3148 & 0.0002 & 0.7045 & -4.3521 & 0.0215 & 0.7650 & -4.3841 & 0.0750 \\
\hline $\mathrm{Cb} 8$ & -0.6606 & -4.2977 & -0.0909 & -0.6612 & -4.3079 & -0.1134 & -0.7320 & -4.3301 & -0.1721 \\
\hline
\end{tabular}


Table 34: Atomic coordinates of the best estimate ( $R B C 4$ ) of the conformation of the $R$. sphaeroides $D M$ macrocycle (angstroms). $\mathrm{n}=32$.

\begin{tabular}{|c|c|c|c|c|c|c|c|c|c|}
\hline \multirow[b]{2}{*}{ Atom } & \multicolumn{3}{|c|}{ Minimum } & \multicolumn{3}{|c|}{ Extended } & \multicolumn{3}{|c|}{ Complete } \\
\hline & $x$ & $y$ & $z$ & $x$ & $y$ & $z$ & $x$ & $y$ & $z$ \\
\hline N1 & -2.0255 & -0.0205 & -0.0368 & -2.0277 & 0.0208 & -0.0370 & -2.0599 & 0.0190 & -0.0410 \\
\hline N2 & -0.0175 & 2.0985 & -0.0570 & 0.0095 & 2.0927 & -0.0571 & 0.0111 & 2.0860 & -0.0599 \\
\hline N3 & 2.0002 & 0.0191 & 0.0111 & 1.9823 & 0.0644 & -0.0007 & 2.0051 & 0.0650 & -0.0019 \\
\hline N4 & 0.0199 & -2.0833 & -0.0460 & 0.0438 & -2.1112 & -0.0361 & 0.0361 & -2.0960 & -0.0290 \\
\hline $\mathrm{Cm} 1$ & -2.4267 & 2.4539 & -0.1880 & -2.4396 & 2.4252 & -0.1768 & -2.4209 & 2.4310 & -0.1820 \\
\hline $\mathrm{Cm} 2$ & 2.4247 & 2.4524 & 0.1747 & 2.4615 & 2.4561 & 0.1689 & 2.4370 & 2.4460 & 0.1711 \\
\hline $\mathrm{Cm} 3$ & 2.4251 & -2.4471 & -0.1331 & 2.4121 & -2.4079 & -0.1212 & 2.4381 & -2.3960 & -0.1310 \\
\hline $\mathrm{Cm} 4$ & -2.4153 & -2.4639 & 0.1402 & -2.3800 & -2.3996 & 0.1270 & -2.3889 & -2.3990 & 0.1390 \\
\hline Ca1 & -2.8562 & -1.1274 & 0.0999 & -2.8363 & -1.0757 & 0.1096 & -2.8409 & -1.0950 & 0.1030 \\
\hline $\mathrm{Ca} 2$ & -2.8383 & 1.1011 & -0.0629 & -2.8754 & 1.0964 & -0.0728 & -2.8849 & 1.1240 & -0.0640 \\
\hline Ca3 & -1.1064 & 2.9430 & -0.1785 & -1.1097 & 2.9075 & -0.1726 & -1.0980 & 2.8760 & -0.1440 \\
\hline $\mathrm{Ca} 4$ & 1.1000 & 2.9122 & 0.0828 & 1.1268 & 2.9222 & 0.0780 & 1.1301 & 2.8920 & 0.0561 \\
\hline $\mathrm{Ca} 5$ & 2.8422 & 1.1129 & 0.1092 & 2.8671 & 1.1164 & 0.1179 & 2.8811 & 1.1140 & 0.1101 \\
\hline $\mathrm{Ca} 6$ & 2.8139 & -1.1004 & -0.0353 & 2.7712 & -1.0605 & -0.0535 & 2.7651 & -1.0470 & -0.0370 \\
\hline $\mathrm{Ca} 7$ & 1.1132 & -2.9227 & -0.1340 & 1.1259 & -2.9532 & -0.1197 & 1.1471 & -2.9230 & -0.1329 \\
\hline $\mathrm{Ca} 8$ & -1.0840 & -2.9093 & 0.0467 & -1.0669 & -2.9123 & 0.0414 & -1.0809 & -2.8750 & 0.0390 \\
\hline $\mathrm{Cb} 1$ & -4.2373 & -0.6848 & 0.1903 & -4.2369 & -0.6850 & 0.1903 & -4.2049 & -0.7030 & 0.1920 \\
\hline $\mathrm{Cb} 2$ & -4.2218 & 0.6779 & 0.0942 & -4.2629 & 0.6693 & 0.0952 & -4.2449 & 0.6740 & 0.0909 \\
\hline $\mathrm{Cb} 3$ & -0.6538 & 4.3338 & -0.1451 & -0.6789 & 4.3024 & -0.1677 & -0.7349 & 4.3280 & -0.2000 \\
\hline $\mathrm{Cb} 4$ & 0.7090 & 4.3080 & 0.0433 & 0.6902 & 4.3112 & 0.0636 & 0.7330 & 4.3390 & 0.0941 \\
\hline Cb5 & 4.2183 & 0.6594 & 0.1192 & 4.2371 & 0.6232 & 0.1242 & 4.2211 & 0.6290 & 0.1321 \\
\hline Cb6 & 4.1946 & -0.6989 & 0.0363 & 4.1633 & -0.7263 & 0.0358 & 4.1431 & -0.7460 & 0.0271 \\
\hline $\mathrm{Cb} 7$ & 0.6908 & -4.3130 & -0.1311 & 0.6954 & -4.3536 & -0.1515 & 0.7671 & -4.3880 & -0.1290 \\
\hline $\mathrm{Cb} 8$ & -0.6693 & -4.3008 & -0.0001 & -0.6717 & -4.3223 & 0.0148 & -0.7559 & -4.3550 & -0.0030 \\
\hline
\end{tabular}

5.9.2 Blastochloris viridis bacteriochlorophylls - BBC1

Table 35: Atomic coordinates of the best estimate of the conformation of the B.viridis BA macrocycle (angstroms); $\mathrm{n}=13$.

\begin{tabular}{|c|c|c|c|c|c|c|c|c|c|}
\hline \multirow[b]{2}{*}{ Atom } & \multicolumn{3}{|c|}{ Minimum } & \multicolumn{3}{|c|}{ Extended } & \multicolumn{3}{|c|}{ Complete } \\
\hline & $x$ & $y$ & $z$ & $x$ & $y$ & $z$ & $x$ & y & $z$ \\
\hline N1 & -2.0231 & -0.0432 & -0.0973 & -2.0189 & -0.0088 & -0.0423 & -2.0310 & -0.0041 & -0.0600 \\
\hline N2 & -0.0005 & 2.1162 & -0.0092 & 0.0168 & 2.1130 & -0.0383 & 0.0270 & 2.1050 & -0.0599 \\
\hline N3 & 1.9734 & 0.0402 & 0.0815 & 1.9564 & 0.0763 & 0.0449 & 1.9719 & 0.0800 & 0.0621 \\
\hline N4 & 0.0052 & -2.0844 & -0.0411 & 0.0203 & -2.1090 & -0.0999 & 0.0210 & -2.1121 & -0.1170 \\
\hline $\mathrm{Cm} 1$ & -2.4065 & 2.4444 & -0.0258 & -2.4374 & 2.4342 & -0.0312 & -2.4191 & 2.4480 & 0.0091 \\
\hline $\mathrm{Cm} 2$ & 2.4387 & 2.4665 & 0.0541 & 2.4855 & 2.4760 & 0.1111 & 2.4599 & 2.4660 & 0.0571 \\
\hline $\mathrm{Cm} 3$ & 2.4028 & -2.4314 & 0.1112 & 2.4086 & -2.4075 & 0.1494 & 2.4450 & -2.4021 & 0.0980 \\
\hline $\mathrm{Cm} 4$ & -2.4195 & -2.4894 & -0.1426 & -2.4118 & -2.4485 & -0.1082 & -2.4120 & -2.4551 & -0.0800 \\
\hline $\mathrm{Ca} 1$ & -2.8578 & -1.1511 & -0.0445 & -2.8385 & -1.1133 & -0.0149 & -2.8360 & -1.1221 & -0.0110 \\
\hline $\mathrm{Ca} 2$ & -2.8242 & 1.0862 & -0.0008 & -2.8519 & 1.0866 & 0.0332 & -2.8481 & 1.1009 & 0.0301 \\
\hline $\mathrm{Ca} 3$ & -1.0885 & 2.9567 & -0.0472 & -1.1045 & 2.9324 & -0.0723 & -1.0911 & 2.9100 & -0.0309 \\
\hline $\mathrm{Ca} 4$ & 1.1185 & 2.9319 & 0.0174 & 1.1501 & 2.9352 & 0.0331 & 1.1400 & 2.9370 & 0.0391 \\
\hline Ca5 & 2.8310 & 1.1217 & 0.0513 & 2.8511 & 1.1272 & 0.0331 & 2.8559 & 1.1220 & 0.0441 \\
\hline $\mathrm{Ca} 6$ & 2.7755 & -1.0855 & 0.0412 & 2.7415 & -1.0555 & 0.0503 & 2.7229 & -1.0470 & 0.0480 \\
\hline $\mathrm{Ca} 7$ & 1.1014 & -2.9154 & 0.0908 & 1.1204 & -2.9387 & 0.0901 & 1.1380 & -2.9040 & 0.0839 \\
\hline
\end{tabular}


Minimum

Extended

Complete

\begin{tabular}{lccccccccc} 
Atom & $\mathrm{x}$ & $\mathrm{y}$ & $\mathrm{z}$ & $\mathrm{x}$ & $\mathrm{y}$ & $\mathrm{z}$ & $\mathrm{x}$ & $\mathrm{y}$ \\
\hline Ca8 & -1.0864 & -2.9249 & -0.1452 & -1.0872 & -2.9290 & -0.1775 & -1.1110 & -2.9031 & -0.1280 \\
Cb1 & -4.2356 & -0.7017 & 0.1627 & -4.2255 & -0.7054 & 0.1351 & -4.2200 & -0.7241 & 0.1300 \\
Cb2 & -4.2101 & 0.6626 & 0.1800 & -4.2341 & 0.6509 & 0.1617 & -4.2330 & 0.6539 & 0.1491 \\
Cb3 & -0.6296 & 4.3521 & -0.0644 & -0.6472 & 4.3240 & -0.0629 & -0.6991 & 4.3489 & -0.1689 \\
Cb4 & 0.7344 & 4.3281 & -0.0274 & 0.7250 & 4.3208 & -0.0336 & 0.7669 & 4.3160 & 0.0582 \\
Cb5 & 4.1988 & 0.6491 & -0.0590 & 4.2088 & 0.6235 & -0.0836 & 4.2130 & 0.6300 & -0.0839 \\
Cb6 & 4.1562 & -0.7065 & -0.0800 & 4.1261 & -0.7236 & -0.0756 & 4.1100 & -0.7409 & -0.0569 \\
Cb7 & 0.7024 & -4.3097 & 0.0857 & 0.7067 & -4.3358 & 0.1225 & 0.7320 & -4.3501 & 0.2219 \\
Cb8 & -0.6561 & -4.3125 & -0.0914 & -0.6605 & -4.3249 & -0.1243 & -0.7030 & -4.3530 & -0.2341 \\
\hline
\end{tabular}

Table 36: Atomic coordinates of the best estimate of the conformation of the B.viridis BB macrocycle (angstroms); $\mathbf{n}=13$.

\begin{tabular}{|c|c|c|c|c|c|c|c|c|c|}
\hline \multirow[b]{2}{*}{ Atom } & \multicolumn{3}{|c|}{ Minimum } & \multicolumn{3}{|c|}{ Extended } & \multicolumn{3}{|c|}{ Complete } \\
\hline & $x$ & y & $z$ & $x$ & $y$ & $z$ & $x$ & y & $z$ \\
\hline N1 & -2.0204 & -0.0363 & -0.1362 & -2.0149 & -0.0044 & -0.0351 & -2.0309 & 0.0039 & -0.0510 \\
\hline N2 & -0.0075 & 2.1059 & -0.0380 & 0.0079 & 2.1033 & -0.0548 & 0.0191 & 2.0989 & -0.0720 \\
\hline N3 & 1.9849 & 0.0352 & 0.0349 & 1.9693 & 0.0712 & -0.0503 & 1.9821 & 0.0839 & -0.0411 \\
\hline N4 & 0.0108 & -2.0940 & -0.0699 & 0.0211 & -2.1181 & -0.0928 & 0.0210 & -2.1190 & -0.1040 \\
\hline $\mathrm{Cm} 1$ & -2.4111 & 2.4485 & -0.0546 & -2.4410 & 2.4388 & -0.0899 & -2.4160 & 2.4479 & -0.0521 \\
\hline $\mathrm{Cm} 2$ & 2.4308 & 2.4656 & 0.0794 & 2.4761 & 2.4724 & 0.1323 & 2.4501 & 2.4590 & 0.0748 \\
\hline $\mathrm{Cm} 3$ & 2.4123 & -2.4375 & 0.0752 & 2.4154 & -2.4114 & 0.1085 & 2.4581 & -2.4120 & 0.0610 \\
\hline $\mathrm{Cm} 4$ & -2.4210 & -2.4802 & -0.1101 & -2.4163 & -2.4418 & -0.1211 & -2.4189 & -2.4480 & -0.0870 \\
\hline Ca1 & -2.8576 & -1.1403 & -0.0627 & -2.8413 & -1.1024 & -0.0194 & -2.8399 & -1.1091 & -0.0140 \\
\hline $\mathrm{Ca} 2$ & -2.8227 & 1.0934 & -0.0261 & -2.8451 & 1.0947 & 0.0178 & -2.8440 & 1.1099 & 0.0090 \\
\hline Ca3 & -1.0943 & 2.9491 & -0.0733 & -1.1122 & 2.9257 & -0.1116 & -1.0979 & 2.9060 & -0.0711 \\
\hline $\mathrm{Ca} 4$ & 1.1085 & 2.9232 & 0.0500 & 1.1370 & 2.9265 & 0.0789 & 1.1260 & 2.9250 & 0.0799 \\
\hline Ca5 & 2.8347 & 1.1242 & 0.0300 & 2.8583 & 1.1269 & -0.0030 & 2.8591 & 1.1230 & 0.0119 \\
\hline Ca6 & 2.7917 & -1.0880 & 0.0129 & 2.7565 & -1.0589 & -0.0145 & 2.7391 & -1.0500 & -0.0041 \\
\hline $\mathrm{Ca} 7$ & 1.1067 & -2.9279 & 0.0582 & 1.1246 & -2.9461 & 0.0900 & 1.1441 & -2.9110 & 0.0741 \\
\hline $\mathrm{Ca} 8$ & -1.0888 & -2.9264 & -0.1061 & -1.0906 & -2.9347 & -0.1522 & -1.1139 & -2.9100 & -0.1100 \\
\hline $\mathrm{Cb} 1$ & -4.2353 & -0.6845 & 0.1527 & -4.2271 & -0.6789 & 0.1222 & -4.2199 & -0.6960 & 0.1071 \\
\hline $\mathrm{Cb} 2$ & -4.2080 & 0.6789 & 0.1763 & -4.2271 & 0.6764 & 0.1664 & -4.2229 & 0.6749 & 0.1650 \\
\hline $\mathrm{Cb} 3$ & -0.6412 & 4.3425 & -0.0301 & -0.6674 & 4.3155 & -0.0494 & -0.7130 & 4.3430 & -0.1551 \\
\hline Cb4 & 0.7214 & 4.3185 & 0.0567 & 0.7033 & 4.3119 & 0.0790 & 0.7351 & 4.3030 & 0.1738 \\
\hline Cb5 & 4.2061 & 0.6633 & -0.0586 & 4.2203 & 0.6296 & -0.0642 & 4.2261 & 0.6420 & -0.0561 \\
\hline Cb6 & 4.1724 & -0.6938 & -0.0733 & 4.1423 & -0.7192 & -0.0540 & 4.1271 & -0.7451 & -0.0491 \\
\hline $\mathrm{Cb} 7$ & 0.6941 & -4.3221 & 0.1154 & 0.7100 & -4.3442 & 0.1590 & 0.7371 & -4.3530 & 0.2611 \\
\hline $\mathrm{Cb} 8$ & -0.6664 & -4.3172 & -0.0027 & -0.6591 & -4.3327 & -0.0419 & -0.7069 & -4.3671 & -0.1510 \\
\hline
\end{tabular}

Table 37: Atomic coordinates of the best estimate of the conformation of the B. viridis DL macrocycle (angstroms); $n=13$.

\begin{tabular}{lccccccrrrr}
\hline & \multicolumn{3}{c}{ Minimum } & \multicolumn{3}{c}{ Extended } & \multicolumn{3}{c}{ Complete } \\
Atom & $\mathrm{x}$ & $\mathrm{y}$ & $\mathrm{z}$ & $\mathrm{x}$ & $\mathrm{y}$ & $\mathrm{z}$ & $\mathrm{x}$ & $\mathrm{y}$ & -0.0399 \\
\hline N1 & -2.0343 & -0.0394 & -0.0276 & -2.0301 & 0.0000 & -0.0430 & -2.0419 & 0.0050 \\
N2 & -0.0085 & 2.0966 & 0.0215 & 0.0104 & 2.0936 & 0.0006 & 0.0251 & 2.0861 & -0.0020 \\
N3 & 1.9783 & 0.0380 & -0.0310 & 1.9605 & 0.0760 & 0.0175 & 1.9750 & 0.0831 & 0.0000 \\
N4 & 0.0138 & -2.0820 & -0.0097 & 0.0295 & -2.1093 & -0.0173 & 0.0220 & -2.1050 & -0.0051 \\
\hline
\end{tabular}


Minimum

Extended

Complete

\begin{tabular}{|c|c|c|c|c|c|c|c|c|c|}
\hline Atom & $x$ & $y$ & z & $x$ & $y$ & z & $x$ & $y$ & $z$ \\
\hline $\mathrm{Cm} 1$ & -2.4113 & 2.4466 & -0.2244 & -2.4359 & 2.4314 & -0.1772 & -2.4210 & 2.4420 & -0.2118 \\
\hline $\mathrm{Cm} 2$ & 2.4341 & 2.4579 & 0.2524 & 2.4801 & 2.4580 & 0.2049 & 2.4570 & 2.4430 & 0.2380 \\
\hline $\mathrm{Cm} 3$ & 2.4146 & -2.4286 & -0.2567 & 2.4112 & -2.3979 & -0.2368 & 2.4510 & -2.3899 & -0.2311 \\
\hline $\mathrm{Cm} 4$ & -2.4201 & -2.4804 & 0.2265 & -2.4067 & -2.4258 & 0.1977 & -2.4090 & -2.4380 & 0.1890 \\
\hline Ca1 & -2.8709 & -1.1436 & 0.0474 & -2.8511 & -1.0980 & 0.0703 & -2.8450 & -1.1080 & 0.0670 \\
\hline $\mathrm{Ca} 2$ & -2.8337 & 1.0924 & -0.1195 & -2.8628 & 1.0898 & -0.1541 & -2.8640 & 1.1030 & -0.1389 \\
\hline Ca3 & -1.0907 & 2.9437 & -0.1486 & -1.1026 & 2.9220 & -0.1285 & -1.0900 & 2.8980 & -0.1098 \\
\hline $\mathrm{Ca} 4$ & 1.1125 & 2.9083 & 0.2210 & 1.1450 & 2.9074 & 0.1814 & 1.1340 & 2.9090 & 0.1691 \\
\hline $\mathrm{Ca} 5$ & 2.8333 & 1.1201 & 0.0723 & 2.8572 & 1.1217 & 0.1253 & 2.8580 & 1.1180 & 0.1080 \\
\hline Ca6 & 2.7861 & -1.0821 & -0.1471 & 2.7478 & -1.0459 & -0.1611 & 2.7311 & -1.0379 & -0.1241 \\
\hline $\mathrm{Ca} 7$ & 1.1116 & -2.9133 & -0.1635 & 1.1263 & -2.9430 & -0.1632 & 1.1421 & -2.9090 & -0.1551 \\
\hline $\mathrm{Ca} 8$ & -1.0828 & -2.9165 & 0.2119 & -1.0819 & -2.9173 & 0.2026 & -1.1029 & -2.8940 & 0.1689 \\
\hline $\mathrm{Cb} 1$ & -4.2504 & -0.6864 & -0.0315 & -4.2437 & -0.6996 & -0.0211 & -4.2339 & -0.7120 & -0.0159 \\
\hline $\mathrm{Cb} 2$ & -4.2211 & 0.6784 & -0.1074 & -4.2491 & 0.6559 & -0.1172 & -4.2500 & 0.6600 & -0.1169 \\
\hline $\mathrm{Cb} 3$ & -0.6242 & 4.3347 & -0.0748 & -0.6411 & 4.3114 & -0.1159 & -0.6870 & 4.3421 & -0.0889 \\
\hline $\mathrm{Cb} 4$ & 0.7380 & 4.3030 & 0.2048 & 0.7295 & 4.2939 & 0.2595 & 0.7650 & 4.2870 & 0.2301 \\
\hline $\mathrm{Cb} 5$ & 4.2032 & 0.6592 & -0.0083 & 4.2193 & 0.6402 & -0.0028 & 4.2241 & 0.6431 & 0.0079 \\
\hline $\mathrm{Cb} 6$ & 4.1660 & -0.6957 & -0.1215 & 4.1352 & -0.7051 & -0.1445 & 4.1210 & -0.7189 & -0.1702 \\
\hline $\mathrm{Cb} 7$ & 0.7081 & -4.3063 & -0.0349 & 0.7109 & -4.3417 & -0.0998 & 0.7231 & -4.3740 & -0.1442 \\
\hline $\mathrm{Cb} 8$ & -0.6515 & -4.3047 & 0.2489 & -0.6578 & -4.3178 & 0.3227 & -0.6839 & -4.3329 & 0.3759 \\
\hline
\end{tabular}

Table 38: Atomic coordinates of the best estimate of the conformation of the B.viridis DM macrocycle (angstroms); $\mathrm{n}=13$.

\begin{tabular}{|c|c|c|c|c|c|c|c|c|c|}
\hline \multirow[b]{2}{*}{ Atom } & \multicolumn{3}{|c|}{ Minimum } & \multicolumn{3}{|c|}{ Extended } & \multicolumn{3}{|c|}{ Complete } \\
\hline & $x$ & y & $z$ & $x$ & $y$ & $z$ & $x$ & y & $z$ \\
\hline N1 & -2.0301 & -0.0342 & -0.0348 & -2.0272 & 0.0055 & -0.0413 & -2.0420 & 0.0100 & -0.0450 \\
\hline N2 & -0.0076 & 2.0803 & 0.0280 & 0.0067 & 2.0755 & 0.0286 & 0.0160 & 2.0760 & 0.0102 \\
\hline N3 & 1.9762 & 0.0327 & -0.0032 & 1.9632 & 0.0725 & 0.0241 & 1.9770 & 0.0749 & 0.0102 \\
\hline N4 & 0.0127 & -2.0644 & -0.0433 & 0.0242 & -2.0907 & -0.0602 & 0.0170 & -2.0950 & -0.0370 \\
\hline $\mathrm{Cm} 1$ & -2.4109 & 2.4409 & -0.3202 & -2.4275 & 2.4232 & -0.2775 & -2.4140 & 2.4340 & -0.2909 \\
\hline $\mathrm{Cm} 2$ & 2.4309 & 2.4484 & 0.3855 & 2.4626 & 2.4438 & 0.3308 & 2.4400 & 2.4350 & 0.3493 \\
\hline $\mathrm{Cm} 3$ & 2.4134 & -2.4238 & -0.3573 & 2.3968 & -2.3847 & -0.3198 & 2.4380 & -2.3730 & -0.3389 \\
\hline $\mathrm{Cm} 4$ & -2.4168 & -2.4703 & 0.2894 & -2.3919 & -2.4151 & 0.2540 & -2.4000 & -2.4240 & 0.2559 \\
\hline Ca1 & -2.8646 & -1.1368 & 0.0905 & -2.8425 & -1.0911 & 0.1280 & -2.8370 & -1.0970 & 0.1309 \\
\hline $\mathrm{Ca} 2$ & -2.8335 & 1.0911 & -0.1316 & -2.8634 & 1.0890 & -0.1746 & -2.8630 & 1.0990 & -0.1670 \\
\hline $\mathrm{Ca} 3$ & -1.0894 & 2.9258 & -0.2499 & -1.0971 & 2.9050 & -0.2241 & -1.0829 & 2.8840 & -0.1789 \\
\hline $\mathrm{Ca} 4$ & 1.1101 & 2.8928 & 0.2914 & 1.1319 & 2.8906 & 0.2638 & 1.1120 & 2.9000 & 0.2543 \\
\hline Ca5 & 2.8291 & 1.1133 & 0.1627 & 2.8552 & 1.1143 & 0.2060 & 2.8580 & 1.1060 & 0.1873 \\
\hline Ca6 & 2.7871 & -1.0820 & -0.1795 & 2.7511 & -1.0435 & -0.2014 & 2.7350 & -1.0359 & -0.1528 \\
\hline $\mathrm{Ca} 7$ & 1.1089 & -2.8952 & -0.2508 & 1.1144 & -2.9201 & -0.2452 & 1.1250 & -2.8910 & -0.2580 \\
\hline Ca8 & -1.0809 & -2.8992 & 0.2374 & -1.0736 & -2.9058 & 0.2161 & -1.0950 & -2.8800 & 0.1859 \\
\hline $\mathrm{Cb} 1$ & -4.2438 & -0.6874 & 0.0578 & -4.2375 & -0.6973 & 0.0762 & -4.2260 & -0.7100 & 0.0829 \\
\hline $\mathrm{Cb} 2$ & -4.2192 & 0.6760 & -0.0431 & -4.2503 & 0.6580 & -0.0611 & -4.2490 & 0.6580 & -0.0691 \\
\hline Cb3 & -0.6271 & 4.3139 & -0.2154 & -0.6416 & 4.2942 & -0.2757 & -0.6450 & 4.3080 & -0.2988 \\
\hline $\mathrm{Cb} 4$ & 0.7337 & 4.2846 & 0.1899 & 0.7253 & 4.2783 & 0.2564 & 0.7280 & 4.2740 & 0.2653 \\
\hline Cb5 & 4.1989 & 0.6584 & 0.0580 & 4.2211 & 0.6353 & 0.0652 & 4.2310 & 0.6370 & 0.0733 \\
\hline Cb6 & 4.1657 & -0.6954 & -0.1284 & 4.1415 & -0.7102 & -0.1446 & 4.1250 & -0.7240 & -0.1627 \\
\hline
\end{tabular}




\begin{tabular}{lccccccrrr} 
Atom & $\mathrm{x}$ & $\mathrm{y}$ & $\mathrm{z}$ & $\mathrm{x}$ & $\mathrm{y}$ & $\mathrm{z}$ & $\mathrm{x}$ & $\mathrm{y}$ & $\mathrm{z}$ \\
\hline Cb7 & 0.7075 & -4.2849 & -0.1003 & 0.7117 & -4.3203 & -0.1704 & 0.7280 & -4.3510 & -0.1661 \\
Cb8 & -0.6505 & -4.2846 & 0.2672 & -0.6531 & -4.3062 & 0.3467 & -0.6761 & -4.3151 & 0.3599
\end{tabular}

\subsubsection{Rhodobacter sphaeroides bacteriopheophytins - RBP4}

Table 39: Atomic coordinates of the best estimate of the conformation of the ФA macrocycle (RBP4, angstroms); $n=16$.

\begin{tabular}{|c|c|c|c|c|c|c|c|c|c|}
\hline \multirow[b]{2}{*}{ Atom } & \multicolumn{3}{|c|}{ Minimum } & \multicolumn{3}{|c|}{ Extended } & \multicolumn{3}{|c|}{ Complete } \\
\hline & $x$ & $y$ & $z$ & $x$ & y & $z$ & $x$ & y & $z$ \\
\hline N1 & -2.0206 & -0.0702 & 0.0520 & -2.0312 & -0.0431 & 0.0126 & -2.0640 & -0.0411 & 0.0170 \\
\hline N2 & 0.0188 & 2.1611 & 0.0649 & 0.0639 & 2.1581 & 0.0640 & 0.0530 & 2.1450 & 0.0640 \\
\hline N3 & 1.9820 & 0.0659 & 0.0840 & 1.9612 & 0.0971 & 0.0680 & 1.9970 & 0.0960 & 0.0779 \\
\hline N4 & -0.0152 & -2.1149 & 0.0651 & 0.0204 & -2.1351 & 0.0809 & 0.0131 & -2.1430 & 0.0710 \\
\hline $\mathrm{Cm} 1$ & -2.3942 & 2.4382 & 0.1551 & -2.3825 & 2.4025 & 0.1625 & -2.3620 & 2.3979 & 0.1779 \\
\hline $\mathrm{Cm} 2$ & 2.4618 & 2.5038 & -0.1489 & 2.4985 & 2.5104 & -0.1121 & 2.4750 & 2.5010 & -0.1170 \\
\hline $\mathrm{Cm} 3$ & 2.3834 & -2.4314 & 0.1852 & 2.3755 & -2.4149 & 0.1784 & 2.3962 & -2.4060 & 0.1871 \\
\hline $\mathrm{Cm} 4$ & -2.4391 & -2.5249 & -0.1786 & -2.4064 & -2.4593 & -0.1400 & -2.4179 & -2.4541 & -0.1550 \\
\hline Ca1 & -2.8652 & -1.1795 & -0.0489 & -2.8702 & -1.1324 & -0.0822 & -2.8719 & -1.1461 & -0.0710 \\
\hline $\mathrm{Ca} 2$ & -2.8109 & 1.0705 & 0.0795 & -2.8343 & 1.0632 & 0.0898 & -2.8400 & 1.0919 & 0.0699 \\
\hline $\mathrm{Ca} 3$ & -1.0814 & 2.9898 & 0.1347 & -1.0696 & 2.9495 & 0.1336 & -1.0611 & 2.9039 & 0.1129 \\
\hline $\mathrm{Ca} 4$ & 1.1376 & 2.9870 & -0.1033 & 1.1659 & 3.0106 & -0.0907 & 1.1699 & 2.9800 & -0.0730 \\
\hline $\mathrm{Ca} 5$ & 2.8518 & 1.1470 & -0.0367 & 2.8710 & 1.1465 & -0.0540 & 2.8920 & 1.1501 & -0.0510 \\
\hline Ca6 & 2.7657 & -1.0797 & 0.0920 & 2.7198 & -1.0578 & 0.1048 & 2.7071 & -1.0590 & 0.0791 \\
\hline $\mathrm{Ca} 7$ & 1.0848 & -2.9415 & 0.1618 & 1.1037 & -2.9793 & 0.1584 & 1.1261 & -2.9470 & 0.1590 \\
\hline $\mathrm{Ca} 8$ & -1.1062 & -2.9651 & -0.1302 & -1.0810 & -2.9511 & -0.1021 & -1.0879 & -2.9261 & -0.0800 \\
\hline $\mathrm{Cb} 1$ & -4.2397 & -0.7181 & -0.0551 & -4.2632 & -0.7059 & -0.0603 & -4.2369 & -0.7162 & -0.0641 \\
\hline $\mathrm{Cb} 2$ & -4.2009 & 0.6479 & 0.0044 & -4.2345 & 0.6539 & 0.0134 & -4.2170 & 0.6508 & 0.0199 \\
\hline $\mathrm{Cb} 3$ & -0.6329 & 4.3969 & 0.0315 & -0.6838 & 4.3665 & 0.0581 & -0.7611 & 4.4009 & 0.0719 \\
\hline $\mathrm{Cb} 4$ & 0.7355 & 4.3867 & -0.1513 & 0.6889 & 4.4030 & -0.1978 & 0.7629 & 4.4430 & -0.2121 \\
\hline $\mathrm{Cb} 5$ & 4.2169 & 0.6445 & -0.0984 & 4.2291 & 0.6083 & -0.1014 & 4.2090 & 0.6232 & -0.1099 \\
\hline $\mathrm{Cb} 6$ & 4.1532 & -0.7147 & -0.0387 & 4.1142 & -0.7440 & -0.0391 & 4.0841 & -0.7639 & -0.0239 \\
\hline $\mathrm{Cb} 7$ & 0.6874 & -4.3444 & 0.0505 & 0.7049 & -4.3874 & 0.0672 & 0.7632 & -4.4050 & 0.0671 \\
\hline $\mathrm{Cb} 8$ & -0.6728 & -4.3549 & -0.1708 & -0.6602 & -4.3594 & -0.2119 & -0.7288 & -4.3761 & -0.2179 \\
\hline
\end{tabular}

Table 40: Atomic coordinates of the best estimate of the conformation of the ФB macrocycle (RBP4, angstroms); $n=16$.

\begin{tabular}{lccccccccc}
\hline & \multicolumn{3}{c}{ Minimum } & \multicolumn{3}{c}{ Extended } & \multicolumn{3}{c}{ Complete } \\
Atom & $\mathrm{x}$ & $\mathrm{y}$ & $\mathrm{z}$ & $\mathrm{x}$ & $\mathrm{y}$ & $\mathrm{z}$ & $\mathrm{x}$ & $\mathrm{y}$ \\
\hline $\mathrm{N} 1$ & -2.0084 & -0.0730 & 0.0400 & -2.0203 & -0.0430 & 0.0286 & -2.0559 & -0.0349 & 0.0270 \\
$\mathrm{~N} 2$ & 0.0236 & 2.1799 & 0.0596 & 0.0721 & 2.1752 & 0.0616 & 0.0671 & 2.1760 & 0.0640 \\
$\mathrm{~N} 3$ & 1.9922 & 0.0677 & 0.0931 & 1.9715 & 0.1037 & 0.0468 & 2.0081 & 0.1010 & 0.0581 \\
$\mathrm{~N} 4$ & -0.0220 & -2.1235 & 0.0419 & 0.0128 & -2.1448 & 0.0546 & 0.0080 & -2.1550 & 0.0421 \\
Cm1 & -2.3941 & 2.4363 & 0.1332 & -2.3812 & 2.4030 & 0.1371 & -2.3699 & 2.3931 & 0.1599 \\
Cm2 & 2.4656 & 2.5161 & -0.1110 & 2.5066 & 2.5261 & -0.0707 & 2.4821 & 2.5170 & -0.0780 \\
Cm3 & 2.3762 & -2.4392 & 0.1662 & 2.3638 & -2.4231 & 0.1786 & 2.3730 & -2.4140 & 0.1751 \\
Cm4 & -2.4427 & -2.5307 & -0.1771 & -2.4085 & -2.4635 & -0.1537 & -2.4160 & -2.4639 & -0.1660 \\
Ca1 & -2.8541 & -1.1834 & -0.0504 & -2.8614 & -1.1331 & -0.0651 & -2.8609 & -1.1539 & -0.0499 \\
Ca2 & -2.8015 & 1.0654 & 0.0928 & -2.8239 & 1.0627 & 0.1072 & -2.8309 & 1.0901 & 0.0900 \\
Ca3 & -1.0850 & 3.0011 & 0.0915 & -1.0732 & 2.9567 & 0.0839 & -1.0639 & 2.9141 & 0.0678 \\
\hline
\end{tabular}




\begin{tabular}{llllllllll} 
Atom & $\mathrm{x}$ & $\mathrm{y}$ & $\mathrm{z}$ & $\mathrm{x}$ & $\mathrm{y}$ & $\mathrm{z}$ & $\mathrm{x}$ & $\mathrm{y}$ \\
\hline Ca4 & 1.1390 & 3.0118 & -0.0818 & 1.1674 & 3.0373 & -0.0600 & 1.1731 & 3.0080 & -0.0531 \\
Ca5 & 2.8612 & 1.1530 & -0.0150 & 2.8874 & 1.1539 & -0.0391 & 2.9061 & 1.1560 & -0.0380 \\
Ca6 & 2.7698 & -1.0859 & 0.0984 & 2.7163 & -1.0652 & 0.0944 & 2.7051 & -1.0630 & 0.0751 \\
Ca7 & 1.0753 & -2.9527 & 0.1293 & 1.0939 & -2.9909 & 0.1398 & 1.1140 & -2.9610 & 0.1402 \\
Ca8 & -1.1146 & -2.9745 & -0.1333 & -1.0864 & -2.9629 & -0.1210 & -1.0950 & -2.9329 & -0.0908 \\
Cb1 & -4.2268 & -0.7271 & -0.0165 & -4.2530 & -0.7072 & -0.0229 & -4.2279 & -0.7159 & -0.0270 \\
Cb2 & -4.1900 & 0.6377 & 0.0625 & -4.2227 & 0.6519 & 0.0641 & -4.1998 & 0.6551 & 0.0660 \\
Cb3 & -0.6505 & 4.4130 & -0.0285 & -0.7150 & 4.3786 & -0.0239 & -0.7928 & 4.4071 & -0.0291 \\
Cb4 & 0.7198 & 4.4129 & -0.1586 & 0.6592 & 4.4297 & -0.1848 & 0.7342 & 4.4590 & -0.1791 \\
Cb5 & 4.2272 & 0.6400 & -0.0774 & 4.2448 & 0.5925 & -0.0751 & 4.2231 & 0.6050 & -0.0829 \\
Cb6 & 4.1601 & -0.7220 & -0.0197 & 4.1145 & -0.7631 & -0.0171 & 4.0901 & -0.7790 & -0.0009 \\
Cb7 & 0.6701 & -4.3571 & 0.0272 & 0.7002 & -4.4014 & 0.0424 & 0.7590 & -4.4230 & 0.0592 \\
Cb8 & -0.6902 & -4.3658 & -0.1665 & -0.6647 & -4.3732 & -0.2058 & -0.7300 & -4.3850 & -0.2298 \\
\hline
\end{tabular}

5.9.4 Rhodobacter sphaeroides bacteriopheophytins - RBP5

Table 41: Atomic coordinates of the best estimate of the conformation of the ФA macrocycle (RBP5, angstroms); $\mathrm{n}=10$.

\begin{tabular}{|c|c|c|c|c|c|c|c|c|c|}
\hline \multirow[b]{2}{*}{ Atom } & \multicolumn{3}{|c|}{ Minimum } & \multicolumn{3}{|c|}{ Extended } & \multicolumn{3}{|c|}{ Complete } \\
\hline & $x$ & $y$ & $z$ & $x$ & $y$ & z & $x$ & y & $z$ \\
\hline N1 & -2.0051 & -0.0024 & 0.0305 & -2.0048 & 0.0173 & 0.0458 & -2.0270 & 0.0239 & 0.0440 \\
\hline N2 & -0.0230 & 2.1026 & 0.0159 & 0.0140 & 2.1128 & -0.0095 & 0.0169 & 2.0900 & -0.0080 \\
\hline N3 & 1.9711 & 0.0007 & 0.0359 & 1.9423 & 0.0239 & 0.0251 & 1.9470 & 0.0351 & 0.0270 \\
\hline N4 & 0.0262 & -2.0842 & 0.0426 & 0.0611 & -2.1114 & 0.0558 & 0.0591 & -2.0950 & 0.0540 \\
\hline $\mathrm{Cm} 1$ & -2.4247 & 2.4665 & 0.1230 & -2.4231 & 2.4013 & 0.1203 & -2.4051 & 2.4029 & 0.1171 \\
\hline $\mathrm{Cm} 2$ & 2.4063 & 2.4397 & -0.1449 & 2.4371 & 2.4188 & -0.1213 & 2.4270 & 2.4111 & -0.1250 \\
\hline $\mathrm{Cm} 3$ & 2.4233 & -2.4571 & 0.1529 & 2.4300 & -2.4128 & 0.1457 & 2.4402 & -2.4079 & 0.1470 \\
\hline $\mathrm{Cm} 4$ & -2.3944 & -2.4548 & -0.1250 & -2.3682 & -2.3739 & -0.1244 & -2.3759 & -2.3781 & -0.1190 \\
\hline Ca1 & -2.8240 & -1.1159 & -0.0466 & -2.8214 & -1.0585 & -0.0494 & -2.8450 & -1.0670 & -0.0531 \\
\hline $\mathrm{Ca} 2$ & -2.8259 & 1.1121 & 0.0693 & -2.8509 & 1.0783 & 0.0867 & -2.8720 & 1.0969 & 0.0930 \\
\hline Ca3 & -1.1060 & 2.9516 & 0.0892 & -1.1049 & 2.9178 & 0.0755 & -1.1031 & 2.8880 & 0.0760 \\
\hline $\mathrm{Ca} 4$ & 1.0916 & 2.9137 & -0.0894 & 1.1252 & 2.9393 & -0.0944 & 1.1289 & 2.9010 & -0.0961 \\
\hline $\mathrm{Ca} 5$ & 2.8045 & 1.0973 & -0.0669 & 2.8079 & 1.0766 & -0.0651 & 2.8260 & 1.0801 & -0.0640 \\
\hline $\mathrm{Ca} 6$ & 2.7938 & -1.1102 & 0.0939 & 2.7631 & -1.0679 & 0.0859 & 2.7780 & -1.0459 & 0.0821 \\
\hline $\mathrm{Ca} 7$ & 1.1158 & -2.9259 & 0.1041 & 1.1438 & -2.9584 & 0.1157 & 1.1511 & -2.9390 & 0.1180 \\
\hline $\mathrm{Ca} 8$ & -1.0707 & -2.9114 & -0.0836 & -1.0581 & -2.9003 & -0.0834 & -1.0699 & -2.8650 & -0.0840 \\
\hline $\mathrm{Cb} 1$ & -4.2043 & -0.6862 & -0.0468 & -4.2231 & -0.6767 & -0.0650 & -4.1890 & -0.7061 & -0.0660 \\
\hline $\mathrm{Cb} 2$ & -4.2031 & 0.6755 & 0.0207 & -4.2421 & 0.6708 & 0.0304 & -4.2090 & 0.6859 & 0.0300 \\
\hline $\mathrm{Cb} 3$ & -0.6524 & 4.3407 & 0.0480 & -0.6811 & 4.3204 & 0.0561 & -0.7571 & 4.3570 & 0.0540 \\
\hline $\mathrm{Cb} 4$ & 0.7093 & 4.3115 & -0.0791 & 0.6919 & 4.3360 & -0.0809 & 0.7559 & 4.3710 & -0.0771 \\
\hline Cb5 & 4.1782 & 0.6553 & -0.0701 & 4.1820 & 0.6277 & -0.0715 & 4.1380 & 0.6411 & -0.0690 \\
\hline $\mathrm{Cb} 6$ & 4.1672 & -0.7005 & 0.0295 & 4.1457 & -0.7131 & 0.0300 & 4.1520 & -0.7509 & 0.0290 \\
\hline $\mathrm{Cb} 7$ & 0.7024 & -4.3148 & 0.0152 & 0.7019 & -4.3549 & 0.0264 & 0.7651 & -4.3920 & 0.0270 \\
\hline $\mathrm{Cb} 8$ & -0.6560 & -4.3039 & -0.1183 & -0.6682 & -4.3130 & -0.1344 & -0.7318 & -4.3370 & -0.1370 \\
\hline
\end{tabular}

Table 42: Atomic coordinates of the best estimate of the conformation of the ФВ macrocycle (RBP5, angstroms); $n=10$.

Minimum

Atom
Extended

$\mathrm{X}$ y
Complete

y

Z 


\begin{tabular}{|c|c|c|c|c|c|c|c|c|c|}
\hline Atom & $x$ & $y$ & z & $x$ & $y$ & z & $x$ & $y$ & z \\
\hline N1 & -2.0011 & -0.0069 & 0.0479 & -1.9969 & 0.0068 & 0.0559 & -2.0150 & 0.0139 & 0.0540 \\
\hline $\mathrm{N} 2$ & -0.0320 & 2.0930 & 0.0476 & 0.0001 & 2.1068 & 0.0519 & 0.0070 & 2.0789 & 0.0511 \\
\hline N3 & 1.9837 & 0.0048 & 0.0634 & 1.9553 & 0.0231 & 0.0511 & 1.9621 & 0.0290 & 0.0531 \\
\hline N4 & 0.0336 & -2.0713 & 0.0409 & 0.0630 & -2.0977 & 0.0331 & 0.0640 & -2.0771 & 0.0311 \\
\hline $\mathrm{Cm} 1$ & -2.4308 & 2.4590 & 0.1394 & -2.4320 & 2.3912 & 0.1269 & -2.4180 & 2.3969 & 0.1241 \\
\hline $\mathrm{Cm} 2$ & 2.4043 & 2.4371 & -0.1283 & 2.4325 & 2.4094 & -0.1130 & 2.4260 & 2.4069 & -0.1170 \\
\hline $\mathrm{Cm} 3$ & 2.4256 & -2.4561 & 0.1477 & 2.4367 & -2.4085 & 0.1467 & 2.4461 & -2.3931 & 0.1480 \\
\hline $\mathrm{Cm} 4$ & -2.3938 & -2.4467 & -0.1491 & -2.3761 & -2.3698 & -0.1368 & -2.3849 & -2.3751 & -0.1340 \\
\hline Ca1 & -2.8248 & -1.1137 & -0.0541 & -2.8243 & -1.0578 & -0.0514 & -2.8470 & -1.0681 & -0.0500 \\
\hline $\mathrm{Ca} 2$ & -2.8224 & 1.1053 & 0.0978 & -2.8410 & 1.0675 & 0.1034 & -2.8620 & 1.0839 & 0.1060 \\
\hline $\mathrm{Ca} 3$ & -1.1147 & 2.9427 & 0.0911 & -1.1186 & 2.9130 & 0.0899 & -1.1121 & 2.8859 & 0.0890 \\
\hline $\mathrm{Ca} 4$ & 1.0837 & 2.9014 & -0.0822 & 1.1156 & 2.9269 & -0.0758 & 1.1209 & 2.8900 & -0.0709 \\
\hline $\mathrm{Ca} 5$ & 2.8191 & 1.0987 & -0.0425 & 2.8203 & 1.0717 & -0.0525 & 2.8350 & 1.0729 & -0.0539 \\
\hline Ca6 & 2.8018 & -1.1099 & 0.0981 & 2.7764 & -1.0675 & 0.1002 & 2.7950 & -1.0441 & 0.0980 \\
\hline $\mathrm{Ca} 7$ & 1.1160 & -2.9203 & 0.1015 & 1.1457 & -2.9457 & 0.1036 & 1.1541 & -2.9241 & 0.1080 \\
\hline $\mathrm{Ca} 8$ & -1.0694 & -2.8908 & -0.0978 & -1.0628 & -2.8833 & -0.1042 & -1.0720 & -2.8491 & -0.1060 \\
\hline $\mathrm{Cb} 1$ & -4.2043 & -0.6846 & -0.0518 & -4.2229 & -0.6678 & -0.0606 & -4.1940 & -0.6991 & -0.0650 \\
\hline $\mathrm{Cb} 2$ & -4.1989 & 0.6753 & 0.0420 & -4.2316 & 0.6764 & 0.0461 & -4.1990 & 0.6928 & 0.0490 \\
\hline $\mathrm{Cb} 3$ & -0.6627 & 4.3300 & -0.0084 & -0.6926 & 4.3137 & -0.0020 & -0.7661 & 4.3599 & 0.0000 \\
\hline $\mathrm{Cb} 4$ & 0.6987 & 4.2978 & -0.1306 & 0.6814 & 4.3237 & -0.1410 & 0.7360 & 4.3490 & -0.1429 \\
\hline Cb5 & 4.1943 & 0.6501 & -0.0687 & 4.1975 & 0.6200 & -0.0785 & 4.1490 & 0.6310 & -0.0739 \\
\hline Cb6 & 4.1773 & -0.7067 & 0.0170 & 4.1609 & -0.7206 & 0.0280 & 4.1670 & -0.7610 & 0.0251 \\
\hline $\mathrm{Cb} 7$ & 0.6871 & -4.3055 & 0.0100 & 0.6919 & -4.3384 & 0.0250 & 0.7540 & -4.3801 & 0.0220 \\
\hline $\mathrm{Cb} 8$ & -0.6705 & -4.2827 & -0.1308 & -0.6786 & -4.2929 & -0.1462 & -0.7460 & -4.3201 & -0.1450 \\
\hline
\end{tabular}

5.9.5 Blastochloris viridis bacteriopheophytins - BBP1

Table 43: Atomic coordinates of the best estimate of the conformation of the B. viridis ФA macrocycle (BBP1, angstroms); $n=13$.

\begin{tabular}{|c|c|c|c|c|c|c|c|c|c|}
\hline \multirow[b]{2}{*}{ Atom } & \multicolumn{3}{|c|}{ Minimum } & \multicolumn{3}{|c|}{ Extended } & \multicolumn{3}{|c|}{ Complete } \\
\hline & $x$ & y & z & $x$ & $y$ & $z$ & $x$ & y & $z$ \\
\hline N1 & -2.0454 & -0.1085 & -0.0429 & -2.0421 & -0.0745 & -0.0225 & -2.0437 & -0.0767 & -0.0240 \\
\hline N2 & 0.0625 & 2.1091 & 0.0302 & 0.0886 & 2.1087 & 0.0301 & 0.1007 & 2.1146 & 0.0315 \\
\hline N3 & 1.9941 & 0.1070 & 0.0438 & 1.9722 & 0.1450 & 0.0087 & 1.9903 & 0.1623 & 0.0143 \\
\hline N4 & -0.0576 & -2.0938 & 0.0418 & -0.0354 & -2.1216 & 0.0609 & -0.0427 & -2.1263 & 0.0534 \\
\hline $\mathrm{Cm} 1$ & -2.3597 & 2.3935 & 0.0897 & -2.3848 & 2.3689 & 0.0582 & -2.3590 & 2.3821 & 0.0852 \\
\hline $\mathrm{Cm} 2$ & 2.4986 & 2.5226 & -0.0988 & 2.5481 & 2.5282 & -0.0644 & 2.5203 & 2.5132 & -0.0856 \\
\hline $\mathrm{Cm} 3$ & 2.3621 & -2.3773 & 0.1815 & 2.3684 & -2.3475 & 0.1627 & 2.4042 & -2.3595 & 0.1469 \\
\hline $\mathrm{Cm} 4$ & -2.4851 & -2.5435 & -0.1689 & -2.4720 & -2.4890 & -0.1611 & -2.4711 & -2.4944 & -0.1530 \\
\hline Ca1 & -2.9148 & -1.1986 & -0.0935 & -2.8990 & -1.1521 & -0.1124 & -2.8969 & -1.1563 & -0.1180 \\
\hline $\mathrm{Ca} 2$ & -2.8126 & 1.0433 & 0.0026 & -2.8414 & 1.0363 & 0.0356 & -2.8387 & 1.0537 & 0.0289 \\
\hline Ca3 & -1.0428 & 2.9211 & 0.1061 & -1.0557 & 2.8901 & 0.0820 & -1.0576 & 2.8620 & 0.0772 \\
\hline $\mathrm{Ca} 4$ & 1.1683 & 2.9551 & -0.0327 & 1.2042 & 2.9663 & -0.0087 & 1.1893 & 2.9647 & -0.0102 \\
\hline $\mathrm{Ca} 5$ & 2.8811 & 1.1762 & -0.0699 & 2.9006 & 1.1769 & -0.1031 & 2.9056 & 1.1697 & -0.0851 \\
\hline Ca6 & 2.7683 & -1.0347 & 0.0457 & 2.7291 & -0.9998 & 0.0491 & 2.7172 & -0.9917 & 0.0352 \\
\hline $\mathrm{Ca} 7$ & 1.0613 & -2.8919 & 0.1835 & 1.0851 & -2.9203 & 0.1885 & 1.0941 & -2.8890 & 0.1834 \\
\hline $\mathrm{Ca} 8$ & -1.1405 & -2.9611 & -0.1011 & -1.1370 & -2.9621 & -0.0911 & -1.1543 & -2.9326 & -0.0664 \\
\hline Cb1 & -4.2845 & -0.7062 & -0.0555 & -4.2817 & -0.7038 & -0.0654 & -4.2768 & -0.7227 & -0.0588 \\
\hline
\end{tabular}




\begin{tabular}{lllllllllr} 
Atom & $\mathrm{x}$ & $\mathrm{y}$ & $\mathrm{z}$ & $\mathrm{x}$ & $\mathrm{y}$ & $\mathrm{z}$ & $\mathrm{x}$ & $\mathrm{y}$ & $\mathrm{z}$ \\
\hline Cb2 & -4.2141 & 0.6600 & -0.0122 & -4.2433 & 0.6522 & -0.0038 & -4.2508 & 0.6580 & -0.0140 \\
Cb3 & -0.6110 & 4.3268 & 0.1252 & -0.6382 & 4.2961 & 0.1550 & -0.7033 & 4.3089 & 0.1232 \\
Cb4 & 0.7530 & 4.3398 & 0.0162 & 0.7354 & 4.3409 & -0.0150 & 0.8011 & 4.3412 & 0.0176 \\
Cb5 & 4.2418 & 0.6784 & -0.1792 & 4.2520 & 0.6459 & -0.1745 & 4.2569 & 0.6574 & -0.1890 \\
Cb6 & 4.1631 & -0.6787 & -0.1219 & 4.1294 & -0.7007 & -0.1121 & 4.1067 & -0.7162 & -0.0911 \\
Cb7 & 0.6876 & -4.2990 & 0.1614 & 0.6946 & -4.3315 & 0.2071 & 0.7284 & -4.3440 & 0.2462 \\
Cb8 & -0.6737 & -4.3396 & -0.0513 & -0.6770 & -4.3526 & -0.1037 & -0.7198 & -4.3785 & -0.1477 \\
\hline
\end{tabular}

Table 44: Atomic coordinates of the best estimate of the conformation of the B.viridis ФВ macrocycle (BВP1, angstroms); $\mathbf{n}=13$.

\begin{tabular}{|c|c|c|c|c|c|c|c|c|c|}
\hline \multirow[b]{2}{*}{ Atom } & \multicolumn{3}{|c|}{ Minimum } & \multicolumn{3}{|c|}{ Extended } & \multicolumn{3}{|c|}{ Complete } \\
\hline & $x$ & $y$ & $z$ & $x$ & $y$ & $z$ & $x$ & $y$ & $z$ \\
\hline N1 & -2.0050 & -0.0740 & -0.0359 & -2.0019 & -0.0388 & -0.0574 & -2.0019 & -0.0265 & -0.0588 \\
\hline $\mathrm{N} 2$ & 0.0297 & 2.1411 & 0.0189 & 0.0561 & 2.1398 & 0.0136 & 0.0640 & 2.1552 & 0.0107 \\
\hline N3 & 1.9656 & 0.0728 & 0.0400 & 1.9436 & 0.1131 & 0.0289 & 1.9511 & 0.1295 & 0.0303 \\
\hline N4 & -0.0260 & -2.1291 & 0.0255 & -0.0039 & -2.1572 & 0.0479 & -0.0130 & -2.1668 & 0.0412 \\
\hline $\mathrm{Cm} 1$ & -2.3736 & 2.4329 & 0.1440 & -2.4017 & 2.4105 & 0.1140 & -2.3722 & 2.4168 & 0.1330 \\
\hline $\mathrm{Cm} 2$ & 2.4557 & 2.5107 & -0.1490 & 2.5069 & 2.5208 & -0.0954 & 2.4760 & 2.5135 & -0.1212 \\
\hline $\mathrm{Cm} 3$ & 2.3753 & -2.4205 & 0.2210 & 2.3828 & -2.3914 & 0.1776 & 2.4206 & -2.4024 & 0.1793 \\
\hline $\mathrm{Cm} 4$ & -2.4453 & -2.5268 & -0.2136 & -2.4318 & -2.4752 & -0.1663 & -2.4307 & -2.4838 & -0.1703 \\
\hline Ca1 & -2.8573 & -1.1747 & -0.0969 & -2.8400 & -1.1287 & -0.1396 & -2.8423 & -1.1321 & -0.1331 \\
\hline $\mathrm{Ca} 2$ & -2.7868 & 1.0716 & 0.0362 & -2.8169 & 1.0672 & 0.0642 & -2.8124 & 1.0826 & 0.0525 \\
\hline $\mathrm{Ca} 3$ & -1.0651 & 2.9693 & 0.1344 & -1.0796 & 2.9356 & 0.1236 & -1.0808 & 2.9058 & 0.1177 \\
\hline $\mathrm{Ca} 4$ & 1.1365 & 2.9771 & -0.0919 & 1.1725 & 2.9900 & -0.0802 & 1.1544 & 2.9923 & -0.0676 \\
\hline Ca5 & 2.8316 & 1.1574 & -0.0733 & 2.8518 & 1.1604 & -0.1011 & 2.8571 & 1.1576 & -0.0721 \\
\hline Ca6 & 2.7527 & -1.0652 & 0.0710 & 2.7122 & -1.0310 & 0.0923 & 2.7034 & -1.0261 & 0.0707 \\
\hline Ca7 & 1.0792 & -2.9467 & 0.2008 & 1.1041 & -2.9736 & 0.1970 & 1.1131 & -2.9424 & 0.1797 \\
\hline Ca8 & -1.1151 & -2.9815 & -0.1531 & -1.1108 & -2.9853 & -0.1265 & -1.1315 & -2.9561 & -0.0935 \\
\hline $\mathrm{Cb} 1$ & -4.2267 & -0.6948 & -0.0323 & -4.2217 & -0.6882 & -0.0564 & -4.2233 & -0.6999 & -0.0592 \\
\hline $\mathrm{Cb} 2$ & -4.1765 & 0.6699 & 0.0273 & -4.2060 & 0.6667 & 0.0572 & -4.2074 & 0.6608 & 0.0578 \\
\hline Cb3 & -0.6292 & 4.3746 & 0.1267 & -0.6587 & 4.3403 & 0.1883 & -0.7068 & 4.3497 & 0.1720 \\
\hline $\mathrm{Cb} 4$ & 0.7349 & 4.3718 & -0.0495 & 0.7149 & 4.3732 & -0.1186 & 0.7684 & 4.3651 & -0.1035 \\
\hline Cb5 & 4.1940 & 0.6731 & -0.1385 & 4.2036 & 0.6349 & -0.1550 & 4.2115 & 0.6393 & -0.1716 \\
\hline $\mathrm{Cb} 6$ & 4.1373 & -0.6847 & -0.0709 & 4.1019 & -0.7126 & -0.0519 & 4.0859 & -0.7272 & -0.0330 \\
\hline $\mathrm{Cb} 7$ & 0.6880 & -4.3531 & 0.1631 & 0.6974 & -4.3838 & 0.2174 & 0.7255 & -4.3982 & 0.2501 \\
\hline $\mathrm{Cb} 8$ & -0.6740 & -4.3714 & -0.1039 & -0.6747 & -4.3865 & -0.1737 & -0.7089 & -4.4068 & -0.2113 \\
\hline
\end{tabular}




\section{PDB Structures}

The raw data, i.e. the cofactor atomic coordinates, were downloaded in batches by PDB ligand I.D. from the PDB Ligand Expo site. Specifically, every $B C L(B C h l a), B C B(B C h l$ b), BPH (BPheo a) and BPB ( $B P$ heo b) found in the directory as of 18/10/2010 was downloaded in .ipdb format on that day and the BH1 (BPheo a), in structures 2BNP, 2BNS and 2BOZ, cofactors were downloaded on 28/03/2011. Additionally, any cofactor structure that included alternate conformations was recompiled as two separate structures and both were included in the final analysis.

\subsection{Rhodobacter sphaeroides RC crystal structures}

Table 45: Information relating to the PDB structures of the $R$. sphaeroides reaction center that were included in the analysis. [a] OL denotes outlier in relevant factor analysis; [b] structures containing two RCs in asymmetric unit and therefore two measurements of each cofactor; [c] not included in factor analysis because of erroneous, missing or chemically different cofactor (see comments) and [d] not included in relevant factor analysis since structures do not exhibit any reasonable experimental variation.

\begin{tabular}{|c|c|c|c|c|c|c|c|c|c|c|}
\hline PDB ID & Space & $\mathrm{R}$ & Res. & $\mathrm{T} / \mathrm{K}$ & Comments & Key 1 & Key 2 & $\mathrm{RBC}^{\mathrm{a}}$ & $\mathrm{RBP}^{\mathrm{a}}$ & Ref. \\
\hline $1 \mathrm{AIG}{ }^{b}$ & P 43212 & 0.215 & 2.6 & 77 & Light & 1,2 & 1,2 & 4 & 5 & [13] \\
\hline $1 \mathrm{AIJ}{ }^{\mathrm{b}}$ & P 43212 & 0.216 & 2.2 & 77 & Dark & 3,4 & 3,4 & 4 & 5 & [13] \\
\hline $1 \mathrm{DS} 8^{\mathrm{b}}$ & P 43212 & 0.227 & 2.5 & 90 & Dark; $\mathrm{Cd}^{2+} \mathrm{PT}$ inhibitor & 5,6 & 5,6 & 4 & 4,5 & [14] \\
\hline $1 D V 3^{b}$ & P 43212 & 0.226 & 2.5 & 90 & Light; $\mathrm{Cd}^{2+} \mathrm{PT}$ inhibitor & 7,8 & 7,8 & 4 & 4,5 & [14] \\
\hline $1 D V 6^{b}$ & P 43212 & 0.238 & 2.5 & 90 & Dark; $\mathrm{Zn}^{2+} \mathrm{PT}$ inhibitor & 9,10 & 9,10 & 4 & 4,5 & [14] \\
\hline 1E14 & P 3121 & 0.226 & 2.7 & 100 & FM197R/GM203D [BA] & 11 & 11 & 4 & $\mathrm{OL}$ & [15] \\
\hline 1E6D & P 3121 & 0.174 & 2.3 & 298 & WM115F/ FM197R [BA / P] & 12 & 12 & 4 & 4 & [16] \\
\hline $1 \mathrm{~F} 6 \mathrm{~N}$ & P 3121 & 0.221 & 2.8 & 277 & Error in BCL L304 (BA) & $N A^{c}$ & 13 & - & $\mathrm{OL}$ & [17] \\
\hline 1FNP & P 3121 & 0.216 & 2.6 & 277 & PL209Y & 13 & 14 & 4 & $\mathrm{OL}$ & [17] \\
\hline $1 \mathrm{FNQ}$ & P 3121 & 0.217 & 2.6 & 277 & PL209E & 14 & 15 & 4 & $\mathrm{OL}$ & [17] \\
\hline 1JGW & P 3121 & 0.211 & 2.8 & 298 & TM21L & 15 & 16 & $2 \mathrm{~A}$ & 2 & [18] \\
\hline 1JGX & P 3121 & 0.211 & 3.01 & 298 & TM21D & 16 & 17 & $2 \mathrm{~A}$ & 2 & [18] \\
\hline $1 \mathrm{JGY}$ & P 3121 & 0.218 & 2.7 & 298 & YM76F & 17 & 18 & $2 \mathrm{~A}$ & 2 & [18] \\
\hline $1 J G Z$ & P 3121 & 0.215 & 2.7 & 298 & YM76K & 18 & 19 & $2 \mathrm{~A}$ & 2 & [18] \\
\hline $1 \mathrm{JHO}$ & P 3121 & 0.225 & 3.5 & 298 & EL205L & 19 & 20 & $2 \mathrm{~A}$ & 2 & [18] \\
\hline $1 \mathrm{~K} 6 \mathrm{~L}$ & P 3121 & 0.193 & 3.1 & 293 & & $N A^{d}$ & $N A^{d}$ & 1 & 1 & [19] \\
\hline $1 \mathrm{~K} 6 \mathrm{~N}$ & P 3121 & 0.203 & 3.1 & 293 & EL212A/DL213A & $N A^{d}$ & $N A^{d}$ & 1 & 1 & [19] \\
\hline $1 \mathrm{KBY}$ & P 3121 & 0.195 & 2.5 & 298 & HM202L [DM $\rightarrow$ BPheo] & $N A^{c}$ & 21 & - & 2 & [20] \\
\hline 1L9B & P 1211 & 0.22 & 2.4 & 100 & Cytochrome $\mathrm{C}_{2}$ bound & 20 & 22 & $2 B$ & 2 & [21] \\
\hline $1 \mathrm{LgJ}^{\mathrm{b}}$ & P 1211 & 0.248 & 3.25 & 100 & Cytochrome $\mathrm{C}_{2}$ bound & 21,22 & 23,24 & $2 \mathrm{~B}$ & 2 & [21] \\
\hline $1 \mathrm{M} 3 \mathrm{X}$ & P 3121 & 0.185 & 2.55 & 298 & Surface lipids [BA / ФB] & 23 & 25 & $2 \mathrm{~A}$ & 2 & [22] \\
\hline 1MPS & P 3121 & 0.194 & 2.55 & 298 & YM177F/FM197R [P] & 24 & 26 & 4 & $\mathrm{OL}$ & [22] \\
\hline 10GV & P 42212 & 0.214 & 2.35 & 100 & & 25 & 27 & $2 \mathrm{~B}$ & 2 & [23] \\
\hline 1PCR & P 3121 & 0.186 & 2.65 & NA & & 26 & 28 & 4 & $\mathrm{OL}$ & [24] \\
\hline 1PSS & P 212121 & 0.223 & 3.0 & NA & See ref. & 27 & 29 & $\mathrm{OL}$ & 5 & [25] \\
\hline 1PST & P 212121 & 0.218 & 3.0 & NA & See ref. [DM $\rightarrow$ BPheo] & $N A^{c}$ & 30 & - & 5 & [25] \\
\hline 1QOV & P 3121 & 0.169 & 2.1 & 298 & AM260W & 28 & $N A^{d}$ & 4 & 1 & [26] \\
\hline 1RG5 & P 3121 & 0.155 & 2.5 & 298 & $\mathrm{R}-26.1$ & 29 & 31 & 4 & 4 & [27] \\
\hline $1 R G N$ & P 3121 & 0.189 & 2.8 & 100 & $\begin{array}{l}\mathrm{R}-26.1 \text { reconstituted } \\
\text { spheroidene }\end{array}$ & 30 & 32 & 4 & 4 & [27] \\
\hline 1RQK & P 3121 & 0.162 & 2.7 & 298 & $\begin{array}{l}\text { R-26.1 reconstituted with } \\
\text { 3,4-dihydrospheroidene }\end{array}$ & 31 & 33 & 4 & 4 & [27] \\
\hline 1RVJ & P 3121 & 0.218 & 2.75 & 100 & $\begin{array}{l}\mathrm{DL} 213 \mathrm{~N} / \mathrm{RH} 177 \mathrm{H} ; \text { [ФA is BPheo } \\
\text { b] }\end{array}$ & 32 & $N A^{c}$ & $2 B$ & - & [28] \\
\hline $1 \mathrm{RY} 5$ & P 3121 & 0.211 & 2.1 & 292 & $\mathrm{DL213N}$; [ФA is BPheo b] & 33 & $N A^{c}$ & $2 B$ & - & [28] \\
\hline $1 \mathrm{RZH}$ & P 3121 & 0.221 & 1.8 & 100 & $\begin{array}{l}\text { DL213N/RM233C; [ } \Phi \text { A is BPheo } \\
\text { b] }\end{array}$ & 34 & $N A^{c}$ & 4 & - & [28] \\
\hline
\end{tabular}




\begin{tabular}{|c|c|c|c|c|c|c|c|c|c|c|}
\hline PDB ID & Space & $R$ & Res. & $\mathrm{T} / \mathrm{K}$ & Comments & Key 1 & Key 2 & $\mathrm{RBC}^{\mathrm{a}}$ & $\mathrm{RBP}^{\mathrm{a}}$ & Ref. \\
\hline $1 R Z Z^{b}$ & P 43212 & 0.216 & 2.4 & 100 & $\begin{array}{l}\text { DL213N/RM233C; [ФA is BPheo } \\
\text { b] }\end{array}$ & 35,36 & $N A^{c}, 34$ & $2 \mathrm{~B}$ & 2 & [28] \\
\hline $1500^{b}$ & P 43212 & 0.226 & 2.6 & 100 & $\begin{array}{l}\text { DL213N/RM233C; [ФA is BPheo } \\
\text { b] }\end{array}$ & 37,38 & $N A^{c}, 35$ & $2 \mathrm{~B}$ & 2 & [28] \\
\hline 1UMX & P 3121 & 0.224 & 2.8 & 298 & $\mathrm{RM} 267 \mathrm{~L} ;[$ [A/B is BPheo b] & 39 & $N A^{c}$ & 4 & - & [29] \\
\hline 1YF6 & P 3121 & 0.197 & 2.25 & 100 & $\begin{array}{l}\text { Quintuple mutant }[\mathrm{Q}(\mathrm{A}) \\
\text { deficient; } \Phi \mathrm{A} \rightarrow \mathrm{BChl}]\end{array}$ & 40 & $N A^{c}$ & 3 & - & [30] \\
\hline 1YST & P 212121 & 0.234 & 3.0 & NA & Rhodobacter sphaeroides $Y$ & 41 & 36 & $\mathrm{OL}$ & $\mathrm{OL}$ & [31] \\
\hline 1Z9J & P 4222 & 0.299 & 4.5 & 298 & Redox active metal centre & 42 & 37 & $2 \mathrm{~A}$ & 2 & [32] \\
\hline $129 \mathrm{~K}$ & P 4222 & 0.33 & 4.6 & 298 & As above & 43 & 38 & $2 A$ & 2 & [32] \\
\hline 2BNP & P 42212 & 0.21 & 2.7 & 100 & Dark & 44 & 39 & $\mathrm{OL}$ & 3 & [33] \\
\hline 2BNS & P 42212 & 0.209 & 2.5 & 100 & Light & 45 & 40 & $\mathrm{OL}$ & 3 & [33] \\
\hline $2 \mathrm{BOZ}$ & P 3121 & 0.174 & 2.4 & 100 & GM203L [BA] & 46 & 41 & 4 & 4 & [34] \\
\hline 2GMR & P 212121 & 0.215 & 2.5 & 100 & DL210N & 47 & 42 & 4 & 4 & [35] \\
\hline $2 \mathrm{GNU}$ & P 42212 & 0.2 & 2.2 & 100 & & 48 & 43 & $\mathrm{OL}$ & 3 & [36] \\
\hline $2 \mathrm{HG} 3$ & P 3121 & 0.164 & 2.7 & 100 & R-26.1 & 49 & 44 & 4 & 4 & [37] \\
\hline $2 \mathrm{HG} 9$ & P 3121 & 0.179 & 2.45 & 100 & $\mathrm{R}-26.1$ & 50 & 45 & 4 & 4 & [37] \\
\hline $2 \mathrm{HH} 1$ & P 3121 & 0.178 & 2.55 & 100 & $R-26.1$ & 51 & 46 & 4 & 4 & [37] \\
\hline $2 \mathrm{HHK}$ & P 3121 & 0.172 & 2.5 & 100 & $\mathrm{R}-26.1$ & 52 & 47 & 4 & 4 & [37] \\
\hline $2 \mathrm{HIT}$ & P 3121 & 0.182 & 2.75 & NA & $\mathrm{R}-26.1$ & 53 & 48 & 4 & 4 & [37] \\
\hline $2 \mathrm{HJ} 6$ & P 3121 & 0.174 & 3.0 & 100 & $\mathrm{R}-26.1$ & 54 & 49 & 4 & 4 & [37] \\
\hline $2 \mathrm{~J} 8 \mathrm{C}$ & P 3121 & 0.178 & 1.87 & 100 & Dark, pH8 & 55 & 50 & 3 & 3 & [38] \\
\hline $2 J 8 D$ & P 3121 & 0.197 & 2.07 & 100 & Light, pH8 & 56 & 51 & 3 & 3 & [38] \\
\hline $2 \mathrm{JIY}$ & P 3121 & 0.181 & 2.2 & 100 & AM149W [ФВ exclusion] & 57 & $N A^{c}$ & 4 & - & [39] \\
\hline $2 \mathrm{JJO}$ & P 3121 & 0.203 & 2.8 & 100 & AM248W [QA exclusion] & 58 & 52 & 4 & 4 & [39] \\
\hline $2 \mathrm{RCR}$ & P 212121 & & 3.1 & NA & & 59 & 53 & $\mathrm{OL}$ & 5 & [40] \\
\hline 2UWS & P 3121 & 0.202 & 2.9 & 100 & Light, pH6.5 & 60 & 54 & 3 & $\mathrm{OL}$ & [38] \\
\hline 2UWT & P 3121 & 0.196 & 2.5 & 100 & As above & 61 & 55 & 3 & 3 & [38] \\
\hline 2UWU & P 3121 & 0.208 & 2.04 & 100 & Dark, pH6.5 & 62 & 56 & 3 & 3 & [38] \\
\hline 2UWV & P 3121 & 0.225 & 2.13 & 100 & Light pH6.5 & 63 & 57 & 3 & 3 & [38] \\
\hline 2UWW & P 3121 & 0.205 & 2.05 & 100 & Dark, pH6.5 & 64 & 58 & 3 & 3 & [38] \\
\hline $2 U \times 3$ & P 3121 & 0.185 & 2.5 & 100 & Dark, pH9 & 65 & 59 & 3 & 3 & [38] \\
\hline $2 U \times 4$ & P 3121 & 0.19 & 2.51 & 100 & Light, pH9 & 66 & 60 & 3 & 3 & [38] \\
\hline $2 U \times 5$ & P 3121 & 0.202 & 2.21 & 100 & As above & 67 & 61 & 3 & 3 & [38] \\
\hline $2 U X J$ & P 43212 & 0.194 & 2.25 & 100 & Dark, pH10 & 68 & 62 & 3 & 3 & [38] \\
\hline $2 U X K$ & P 43212 & 0.19 & 2.31 & 100 & Light, pH10 & 69 & 63 & 3 & 3 & [38] \\
\hline $2 U X L$ & P 3121 & 0.176 & 2.88 & 100 & Dark, pH10 & 70 & 64 & 3 & 3 & [38] \\
\hline 2UXM & P 3121 & 0.186 & 2.7 & 100 & Light, $\mathrm{pH} 10$ & 71 & 65 & 3 & 3 & [38] \\
\hline $2 W \times 5$ & P 3121 & 0.237 & 2.63 & 100 & FL181R [BB hexacoordinated] & 72 & 66 & 3 & 3 & [41] \\
\hline $3 D S Y$ & P 3121 & 0.191 & 3.0 & 293 & EL212Q & $N A^{d}$ & $N A^{d}$ & 1 & 1 & {$[42]$} \\
\hline 3DTA & P 3121 & 0.199 & 3.2 & 293 & EL212Q, NM44D & $N A^{d}$ & $N A^{d}$ & 1 & 1 & [42] \\
\hline 3DTR & P 3121 & 0.195 & 3.1 & 293 & EL212Q, LL227F & $N A^{d}$ & $N A^{d}$ & 1 & 1 & [42] \\
\hline 3DTS & P 3121 & 0.209 & 3.1 & 293 & EL212Q, DL213A, RM233L & $N A^{d}$ & $N A^{d}$ & 1 & 1 & [42] \\
\hline 3DU2 & P 3121 & 0.192 & 3.1 & 293 & EL212A & $N A^{d}$ & $N A^{d}$ & 1 & 1 & [42] \\
\hline 3DU3 & P 3121 & 0.191 & 2.8 & 273 & EL212Q, DL213A, AM249Y & $N A^{d}$ & $N A^{d}$ & 1 & 1 & [43] \\
\hline $3 D \cup Q$ & P 3121 & 0.209 & 2.7 & 273 & EL212Q, DL213A, NM5D & $N A^{d}$ & $N A^{d}$ & 1 & 1 & [43] \\
\hline $4 R C R$ & P 212121 & 0.227 & 2.8 & NA & $\mathrm{R}-26$ & 73 & 67 & $\mathrm{OL}$ & $\mathrm{OL}$ & [44] \\
\hline
\end{tabular}




\subsection{Blastochloris viridis RC crystal structures}

Table 46: Information relating to the PDB structures of the B. viridis reaction center that were included in the analysis.

\begin{tabular}{|c|c|c|c|c|c|c|c|c|}
\hline PDB ID & Space Group & $\mathrm{R}$ & Res. & $\mathrm{T} / \mathrm{K}$ & Comments & BBC & BBP & Ref. \\
\hline 1DXR & P 43212 & 0.194 & 2.00 & 263 & 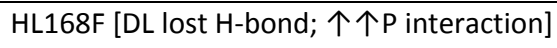 & 2 & 1 & {$[45]$} \\
\hline 1PRC & P 43212 & 0.193 & 2.30 & NA & & 1 & 1 & {$[46]$} \\
\hline $1 \mathrm{R} 2 \mathrm{C}$ & P 43212 & 0.202 & 2.86 & 293 & & 2 & 1 & [47] \\
\hline IVRN & P 43212 & 0.191 & 2.20 & 100 & & 2 & 1 & {$[48]$} \\
\hline $215 \mathrm{~N}$ & P 43212 & 0.173 & 1.96 & NA & & 2 & 1 & [49] \\
\hline $2 J B L$ & P 43212 & 0.190 & 2.40 & 263 & Stigmatellin inhibited & 2 & 1 & {$[50]$} \\
\hline 2PRC & P 43212 & 0.182 & 2.45 & 263 & Ubiquinone-2 complex [QB] & 2 & 1 & {$[51]$} \\
\hline 2WJM & P 21212 & 0.174 & 1.95 & 100 & Lipidic sponge phase & 2 & 1 & {$[52]$} \\
\hline $2 \mathrm{WJN}$ & P 21212 & 0.172 & 1.86 & 100 & As above & 2 & 1 & {$[52]$} \\
\hline 3D38 & P 3121 & 0.194 & 3.21 & 100 & Crystallised with methyl-beta-cyclodextrin & 2 & $\mathrm{OL}$ & {$[53]$} \\
\hline $3 G 7 F$ & P 43212 & 0.177 & 2.50 & 100 & HM200L, [heterodimer (DM)] & NA & $\mathrm{OL}$ & {$[54]$} \\
\hline $3 P R C$ & P 43212 & 0.178 & 2.40 & 263 & QB depleted & 2 & 1 & {$[51]$} \\
\hline 5PRC & P 43212 & 0.190 & 2.35 & 263 & Atrazine complex & 2 & 1 & {$[55]$} \\
\hline $6 \mathrm{PRC}$ & P 43212 & 0.184 & 2.30 & 263 & Triazine complex & 2 & 1 & {$[55]$} \\
\hline 7PRC & P 43212 & 0.184 & 2.65 & 263 & As above & 2 & 1 & {$[55]$} \\
\hline
\end{tabular}




\section{References}

[1] H. M. Berman, J. Westbrook, Z. Feng, G. Gilliland, T. N. Bhat, H. Weissig, I. N. Shindyalov, P. E. Bourne, Nucleic Acids Res 2000, 28, 235-242.

[2] a) W. Jentzen, M. C. Simpson, J. D. Hobbs, X. Song, T. Ema, N. Y. Nelson, C. J. Medforth, K. M. Smith, M. Veyrat, J. Am. Chem. Soc. 1995, 117, 11085-11097; b) W. Jentzen, X. Song, J. A. Shelnutt, J. Phys. Chem. B 1997, 101, 1684-1699; C) W. Jentzen, J. Ma, J. A. Shelnutt, Biophys. J. 1998, 74, 753-763.

[3] S. Lisong, J. A. Shelnutt, 3.0 ed., 2001.

[4] R. D. C. Team, Vienna, Austria, 2010.

[5] J. P. Allen, J. C. Williams, Adv. Photosynth. Respir. 2006, 25, $283-$ 295.

[6] G. Zucchelli, D. Brogioli, A. P. Casazza, F. M. Garlaschi, R. C. Jennings, Biophys. J. 2007, 93, 2240-2254.

[7] M. Gouterman, G. Wagniere, L. C. Snyder, J. Mol. Spectrosc. 1963, 11, 108-127.

[8] E. Gudowska-Nowak, M. D. Newton, J. Fajer, J. Phys. Chem. 1990 94, 5795-5801.

[9] J. Oelze, in Methods in Microbiology, Vol. 18 (Ed.: G. Gottschank), Academic Press, London, 1985, pp. 257-284.

[10] M. O. Senge, M. W. Renner, W. W. Kalisch, J. Fajer, Dalton 2000, 381-385.

[11] B. Roder, M. Buchner, I. Ruckmann, M. O. Senge, Photochem Photobiol Sci 2010, 9, 1152-1158.

[12] C. Olea, J. Kuriyan, M. A. Marletta, J. Am. Chem. Soc. 2010, 132, 12794-12795.

[13] M. H. Stowell, T. M. McPhillips, D. C. Rees, S. M. Soltis, E. Abresch G. Feher, Science 1997, 276, 812-816.

[14] H. L. Axelrod, E. C. Abresch, M. L. Paddock, M. Y. Okamura, G. Feher, Proc Natl Acad Sci U S A 2000, 97, 1542-1547.

[15] P. K. Fyfe, J. P. Ridge, K. E. McAuley, R. J. Cogdell, N. W. Isaacs, M. R. Jones, Biochemistry 2000, 39, 5953-5960.

[16] J. P. Ridge, P. K. Fyfe, K. E. McAuley, M. E. van Brederode, B. Robert, R. van Grondelle, N. W. Isaacs, R. J. Cogdell, M. R. Jones, Biochem J 2000, 351 Pt 3, 567-578.

[17] A. Kuglstatter, U. Ermler, H. Michel, L. Baciou, G. Fritzsch, Biochemistry 2001, 40, 4253-4260.

[18] A. Camara-Artigas, C. L. Magee, J. C. Williams, J. P. Allen, Acto Crystallogr D Biol Crystallogr 2001, 57, 1281-1286.

[19] P. R. Pokkuluri, P. D. Laible, Y. L. Deng, T. N. Wong, D. K. Hanson, M. Schiffer, Biochemistry 2002, 41, 5998-6007.

[20] A. Camara-Artigas, C. Magee, A. Goetsch, J. P. Allen, Photosynth Res 2002, 74, 87-93.

[21] H. L. Axelrod, E. C. Abresch, M. Y. Okamura, A. P. Yeh, D. C. Rees, G. Feher, J Mol Biol 2002, 319, 501-515.

[22] A. Camara-Artigas, D. Brune, J. P. Allen, Proc Natl Acad Sci U S A 2002, 99, 11055-11060.

[23] G. Katona, U. Andreasson, E. M. Landau, L. E. Andreasson, R. Neutze, J Mol Biol 2003, 331, 681-692.

[24] U. Ermler, G. Fritzsch, S. K. Buchanan, H. Michel, Structure 1994, 2, 925-936.

[25] A. J. Chirino, E. J. Lous, M. Huber, J. P. Allen, C. C. Schenck, M. L. Paddock, G. Feher, D. C. Rees, Biochemistry 1994, 33, 4584-4593.

[26] K. E. McAuley, P. K. Fyfe, J. P. Ridge, N. W. Isaacs, R. J. Cogdell, M. R. Jones, Proc Nat/ Acad Sci U S A 1999, 96, 14706-14711.

[27] A. W. Roszak, K. McKendrick, A. T. Gardiner, I. A. Mitchell, N. W. Isaacs, R. J. Cogdell, H. Hashimoto, H. A. Frank, Structure 2004, 12, 765-773.

[28] Q. Xu, H. L. Axelrod, E. C. Abresch, M. L. Paddock, M. Y. Okamura, G. Feher, Structure 2004, 12, 703-715.

[29] P. K. Fyfe, N. W. Isaacs, R. J. Cogdell, M. R. Jones, Biochim Biophys Acta 2004, 1608, 11-22.
[30] M. L. Paddock, C. Chang, Q. Xu, E. C. Abresch, H. L. Axelrod, G. Feher, M. Y. Okamura, Biochemistry 2005, 44, 6920-6928.

[31] B. Arnoux, J. F. Gaucher, A. Ducruix, F. Reiss-Husson, Acta Crystallogr D Biol Crystallogr 1995, 51, 368-379.

[32] M. Thielges, G. Uyeda, A. Camara-Artigas, L. Kalman, J. C. Williams, J. P. Allen, Biochemistry 2005, 44, 7389-7394.

[33] G. Katona, A. Snijder, P. Gourdon, U. Andreasson, O. Hansson, L. E. Andreasson, R. Neutze, Nat Struct Mol Biol 2005, 12, 630-631.

[34] J. A. Potter, P. K. Fyfe, D. Frolov, M. C. Wakeham, R. van Grondelle, B. Robert, M. R. Jones, J Biol Chem 2005, 280, 27155-27164.

[35] S. Hermes, J. M. Stachnik, D. Onidas, A. Remy, E. Hofmann, K. Gerwert, Biochemistry 2006, 45, 13741-13749.

[36] P. Wadsten, A. B. Wohri, A. Snijder, G. Katona, A. T. Gardiner, R. J. Cogdell, R. Neutze, S. Engstrom, J Mol Biol 2006, 364, 44-53.

[37] A. W. Roszak, A. T. Gardiner, N. W. Isaacs, R. J. Cogdell, Biochemistry 2007, 46, 2909-2916.

[38] J. Koepke, E. M. Krammer, A. R. Klingen, P. Sebban, G. M. Ullmann, G. Fritzsch, J Mol Biol 2007, 371, 396-409.

[39] P. K. Fyfe, J. A. Potter, J. Cheng, C. M. Williams, A. J. Watson, M. R. Jones, Biochemistry 2007, 46, 10461-10472.

[40] C. H. Chang, O. el-Kabbani, D. Tiede, J. Norris, M. Schiffer, Biochemistry 1991, 30, 5352-5360.

[41] D. Frolov, M. Marsh, L. I. Crouch, P. K. Fyfe, B. Robert, R. van Grondelle, A. Hadfield, M. R. Jones, Biochemistry 2010, 49, 18821892.

[42] P. R. Pokkuluri, P. D. Laible, D. K. Hanson, M. Schiffer, 2009

[43] P. R. Pokkuluri, P. D. Laible, S. L. Ginell, D. K. Hanson, M. Schiffer, 2009.

[44] T. O. Yeates, H. Komiya, A. Chirino, D. C. Rees, J. P. Allen, G. Feher, Proc Natl Acad Sci U S A 1988, 85, 7993-7997.

[45] C. R. Lancaster, M. V. Bibikova, P. Sabatino, D. Oesterhelt, H. Michel, J Biol Chem 2000, 275, 39364-39368.

[46] J. Deisenhofer, O. Epp, I. Sinning, H. Michel, J Mol Biol 1995, 246, 429-457.

[47] R. H. Baxter, N. Ponomarenko, V. Srajer, R. Pahl, K. Moffat, J. R. Norris, Proc Natl Acad Sci U S A 2004, 101, 5982-5987.

[48] R. H. Baxter, B. L. Seagle, N. Ponomarenko, J. R. Norris, Acta Crystallogr D Biol Crystallogr 2005, 61, 605-612.

[49] L. Li, D. Mustafi, Q. Fu, V. Tereshko, D. L. Chen, J. D. Tice, R. F. Ismagilov, Proc Natl Acad Sci U S A 2006, 103, 19243-19248.

[50] C. R. Lancaster, C. Hunte, J. Kelley, 3rd, B. L. Trumpower, R. Ditchfield, J Mol Biol 2007, 368, 197-208.

[51] C. R. Lancaster, H. Michel, Structure 1997, 5, 1339-1359.

[52] A. B. Wohri, W. Y. Wahlgren, E. Malmerberg, L. C. Johansson, R. Neutze, G. Katona, Biochemistry 2009, 48, 9831-9838.

[53] L. Li, S. Nachtergaele, A. M. Seddon, V. Tereshko, N. Ponomarenko, R. F. Ismagilov, J Am Chem Soc 2008, 130, 14324-14328.

[54] N. S. Ponomarenko, L. Li, A. R. Marino, V. Tereshko, A. Ostafin, J. A. Popova, E. J. Bylina, R. F. Ismagilov, J. R. Norris, Jr., Biochim Biophys Acta 2009, 1788, 1822-1831.

[55] C. R. Lancaster, H. Michel, J Mol Biol 1999, 286, 883-898. 


\section{Cofactor NSDs}

8.1 Rhodobacter sphaeroides bacteriochlorin cofactor NSDs

Table 47: Minimum basis normal-mode displacements and conformational parameters of all BA cofactors from crystal structures of Rhodobacter sphaeroides.

\begin{tabular}{|c|c|c|c|c|c|c|c|c|c|c|c|c|c|c|c|c|}
\hline Cofactor ID & Dip & Sip & B2g & B1g & $\mathrm{Eu}(\mathrm{x})$ & Eu(y) & A1g & A2g & Doop & Soop & $\mathrm{B} 2 \mathrm{u}$ & B1u & $\mathrm{A} 2 \mathrm{u}$ & $\mathrm{Eg}(\mathrm{x})$ & $\mathrm{Eg}(\mathrm{y})$ & Alu \\
\hline 1AIG - BCL L283 & 0.2438 & 0.0511 & -0.1464 & -0.0317 & -0.0548 & -0.0913 & 0.1495 & -0.0577 & 0.2084 & 0.0182 & 0.0474 & 0.0271 & -0.1257 & -0.1475 & 0.0532 & 0.0088 \\
\hline 1AIG - BCL N284 & 0.1836 & 0.0538 & -0.0930 & 0.0513 & -0.0102 & 0.0041 & 0.1292 & -0.0748 & 0.3500 & 0.0215 & 0.2086 & -0.0296 & -0.2045 & -0.1762 & 0.0700 & 0.0173 \\
\hline 1AIJ - BCL L283 & 0.2276 & 0.0504 & -0.0266 & -0.0253 & -0.0311 & -0.0748 & 0.1991 & -0.0655 & 0.2906 & 0.0186 & 0.0390 & 0.1024 & -0.2276 & -0.1321 & 0.0527 & 0.0197 \\
\hline 1AIJ - BCL R283 & 0.2667 & 0.0536 & -0.0683 & -0.0228 & -0.0225 & -0.0626 & 0.2415 & -0.0567 & 0.2422 & 0.0159 & 0.0789 & 0.0780 & -0.1436 & -0.1560 & 0.0362 & 0.0096 \\
\hline 1DS8 - BCL L1004 & 0.2114 & 0.0550 & -0.0806 & -0.0721 & -0.0340 & -0.0665 & 0.1508 & -0.0682 & 0.4044 & 0.0335 & 0.0275 & 0.1393 & -0.2324 & -0.2970 & 0.0321 & 0.0122 \\
\hline 1DS8 - BCL R2004 & 0.1991 & 0.0518 & 0.0422 & -0.0038 & 0.0046 & 0.0128 & 0.1763 & -0.0812 & 0.4173 & 0.0329 & -0.0959 & 0.0701 & -0.2796 & -0.2853 & 0.0100 & 0.0199 \\
\hline 1DV3 - BCL M1004 & 0.2020 & 0.0567 & -0.0810 & 0.0064 & -0.0059 & -0.1032 & 0.1372 & -0.0687 & 0.3817 & 0.0302 & -0.0004 & 0.1161 & -0.2133 & -0.2937 & 0.0105 & 0.0177 \\
\hline 1DV3 - BCL R2004 & 0.2018 & 0.0511 & 0.0263 & -0.0353 & -0.0029 & -0.0264 & 0.1820 & -0.0703 & 0.4227 & 0.0327 & -0.0529 & 0.0768 & -0.3067 & -0.2718 & 0.0201 & 0.0410 \\
\hline 1DV6 - BCL M1004 & 0.2269 & 0.0537 & -0.1174 & -0.0307 & -0.0176 & 0.0134 & 0.1714 & -0.0831 & 0.4116 & 0.0333 & 0.0020 & 0.0957 & -0.2645 & -0.2996 & 0.0221 & 0.0084 \\
\hline 1DV6 - BCL R2004 & 0.2308 & 0.0527 & -0.0341 & -0.0441 & 0.0012 & -0.0968 & 0.1905 & -0.0670 & 0.3700 & 0.0319 & -0.0262 & 0.0496 & -0.2664 & -0.2442 & 0.0322 & 0.0458 \\
\hline 1E14 - BCL C304 & 0.3628 & 0.0531 & 0.0594 & -0.1872 & -0.1495 & 0.0128 & 0.2617 & -0.0456 & 0.4230 & 0.0312 & 0.2734 & -0.0584 & -0.2793 & -0.1494 & -0.0167 & 0.0134 \\
\hline 1E6D - BCL C304 & 0.3886 & 0.0490 & 0.1456 & -0.2406 & -0.0653 & -0.0073 & 0.2497 & -0.0724 & 0.3354 & 0.0265 & 0.0545 & 0.0299 & -0.1113 & -0.3072 & 0.0393 & -0.0178 \\
\hline 1EYS - BCL L604 & 0.3555 & 0.0473 & -0.0100 & -0.2209 & -0.1714 & -0.0821 & 0.1766 & -0.1009 & 0.3242 & 0.0841 & -0.0252 & 0.0741 & -0.0668 & -0.2226 & 0.2103 & 0.0274 \\
\hline 1F6N - BCL L304 & 0.5775 & 0.1251 & -0.4794 & -0.2220 & 0.0024 & -0.0609 & 0.2165 & -0.0616 & 0.4998 & 0.1051 & 0.0812 & 0.0541 & -0.3487 & -0.2869 & 0.1597 & -0.1047 \\
\hline 1FNP - BCL L304 & 0.2985 & 0.0446 & 0.0817 & -0.1718 & -0.0717 & -0.0167 & 0.2088 & -0.0627 & 0.4363 & 0.0737 & 0.1052 & 0.0090 & -0.2267 & -0.3252 & 0.0708 & -0.1306 \\
\hline 1FNQ - BCL L304 & 0.3266 & 0.0431 & 0.0660 & -0.1919 & -0.1009 & -0.0499 & 0.2194 & -0.0684 & 0.3786 & 0.0713 & 0.1821 & 0.0156 & -0.1782 & -0.2610 & -0.0040 & -0.1002 \\
\hline 1JGW - BCL M853 & 0.3162 & 0.0567 & -0.1092 & 0.0099 & -0.1693 & -0.0036 & 0.2412 & -0.0337 & 0.1149 & 0.0066 & 0.0245 & 0.0494 & -0.0864 & -0.0508 & 0.0108 & -0.0010 \\
\hline 1JGX - BCL L853 & 0.3372 & 0.0579 & -0.0818 & -0.0074 & -0.1347 & -0.0234 & 0.2949 & -0.0361 & 0.0948 & 0.0070 & -0.0090 & 0.0341 & -0.0697 & -0.0426 & 0.0322 & 0.0044 \\
\hline 1JGY - BCL M853 & 0.3054 & 0.0587 & -0.1010 & 0.0147 & -0.1770 & -0.0082 & 0.2246 & -0.0322 & 0.0821 & 0.0056 & -0.0551 & 0.0178 & -0.0490 & -0.0270 & 0.0153 & 0.0047 \\
\hline 1JGZ - BCL M853 & 0.3463 & 0.0578 & -0.1271 & -0.0252 & -0.1818 & 0.0208 & 0.2610 & -0.0394 & 0.0965 & 0.0059 & -0.0161 & 0.0643 & -0.0588 & -0.0378 & 0.0061 & -0.0017 \\
\hline 1JHO - BCL L853 & 0.3901 & 0.0589 & -0.1727 & 0.0132 & -0.1988 & 0.0081 & 0.2844 & -0.0418 & 0.0881 & 0.0053 & -0.0016 & 0.0441 & -0.0700 & -0.0238 & 0.0187 & 0.0008 \\
\hline 1K6L - BCL L304 & 0.3538 & 0.0509 & -0.0142 & -0.2130 & -0.1207 & 0.0217 & 0.2460 & -0.0640 & 0.3996 & 0.0676 & -0.0823 & 0.0064 & -0.2593 & -0.1959 & -0.2126 & 0.0455 \\
\hline $1 \mathrm{~K} 6 \mathrm{~N}-\mathrm{BCL}$ L304 & 0.3543 & 0.0509 & -0.0141 & -0.2133 & -0.1204 & 0.0218 & 0.2466 & -0.0636 & 0.3994 & 0.0677 & -0.0827 & 0.0073 & -0.2590 & -0.1954 & -0.2128 & 0.0458 \\
\hline 1KBY - BCL L853 & 0.3604 & 0.0572 & -0.0543 & -0.0105 & -0.1948 & 0.0154 & 0.2960 & -0.0322 & 0.1179 & 0.0063 & 0.0210 & 0.0583 & -0.0858 & -0.0470 & 0.0219 & -0.0025 \\
\hline 1L9B - BCL L1004 & 0.3172 & 0.0672 & 0.0626 & -0.1595 & -0.0413 & -0.0886 & 0.2328 & -0.0865 & 0.2477 & 0.0204 & -0.0052 & -0.0017 & -0.1939 & -0.1394 & 0.0526 & -0.0394 \\
\hline 1L9J - BCL L1004 & 0.3274 & 0.0690 & 0.0649 & -0.1897 & -0.0656 & -0.0925 & 0.2169 & -0.0842 & 0.2517 & 0.0182 & -0.0150 & -0.0442 & -0.1971 & -0.1184 & 0.0831 & -0.0375 \\
\hline 1L9J - BCL R2004 & 0.3469 & 0.0676 & 0.0520 & -0.1871 & -0.0900 & -0.0982 & 0.2376 & -0.0919 & 0.2316 & 0.0145 & -0.0193 & -0.0126 & -0.1918 & -0.1127 & 0.0489 & -0.0356 \\
\hline 1M3X - BCL L853 & 0.2869 & 0.0576 & -0.0562 & 0.0214 & -0.1317 & -0.0388 & 0.2423 & -0.0333 & 0.1197 & 0.0077 & 0.0468 & 0.0607 & -0.0703 & -0.0523 & 0.0276 & -0.0015 \\
\hline 1MPS - BCL L304 & 0.4180 & 0.0378 & 0.0595 & -0.1927 & -0.0773 & -0.0651 & 0.3446 & -0.0717 & 0.3985 & 0.0665 & -0.0181 & 0.1227 & -0.2287 & -0.2995 & -0.0368 & 0.0101 \\
\hline 10GV - BCL L1283 & 0.3790 & 0.0672 & 0.0648 & -0.2053 & -0.0621 & -0.0836 & 0.2779 & -0.0959 & 0.2626 & 0.0244 & -0.0293 & 0.0023 & -0.1911 & -0.1383 & 0.1111 & -0.0092 \\
\hline 1PCR - BCL L304 & 0.3538 & 0.0509 & -0.0162 & -0.2140 & -0.1206 & 0.0215 & 0.2450 & -0.0644 & 0.3995 & 0.0676 & -0.0819 & 0.0051 & -0.2585 & -0.1962 & -0.2132 & 0.0455 \\
\hline 1PSS - BCL M4 & 0.2549 & 0.0511 & 0.0183 & -0.0403 & -0.0639 & -0.1118 & 0.2045 & -0.0679 & 0.2358 & 0.0137 & 0.1027 & 0.1654 & -0.1037 & -0.0763 & -0.0096 & 0.0326 \\
\hline 1PST - BCL L4 & 0.2235 & 0.0512 & -0.0102 & -0.0236 & -0.0722 & -0.0653 & 0.1886 & -0.0650 & 0.3788 & 0.0195 & 0.1329 & 0.2703 & -0.2148 & -0.0663 & 0.0224 & 0.0416 \\
\hline 1PYH - BCL B304 & 0.3416 & 0.0498 & 0.0603 & -0.2854 & -0.1234 & -0.0362 & 0.0902 & -0.0830 & 0.3696 & 0.0339 & -0.0124 & 0.0563 & -0.0969 & -0.3498 & -0.0113 & -0.0373 \\
\hline
\end{tabular}




\begin{tabular}{|c|c|c|c|c|c|c|c|c|c|c|c|c|c|c|c|c|}
\hline Cofactor ID & Dip & Sip & B2g & B1g & $\mathrm{Eu}(\mathrm{x})$ & $\mathrm{Eu}(\mathrm{y})$ & A1g & $A 2 g$ & Doop & Soop & $\mathrm{B} 2 \mathrm{u}$ & B1u & $\mathrm{A} 2 \mathrm{u}$ & $\mathrm{Eg}(\mathrm{x})$ & $\mathrm{Eg}(\mathrm{y})$ & A1u \\
\hline 1QOV - BCL L1304 & 0.3753 & 0.0492 & 0.0943 & -0.2763 & -0.0331 & -0.0127 & 0.2255 & -0.0589 & 0.3965 & 0.0388 & 0.0303 & 0.0440 & -0.1016 & -0.3686 & -0.0667 & -0.0610 \\
\hline 1RG5 - BCL L304 & 0.4007 & 0.0444 & 0.0127 & -0.1858 & -0.1069 & -0.0433 & 0.3246 & -0.0849 & 0.3473 & 0.0316 & -0.0238 & 0.1166 & -0.0986 & -0.3078 & 0.0433 & -0.0093 \\
\hline 1RGN - BCL L304 & 0.3384 & 0.0560 & 0.0914 & -0.1629 & -0.2087 & -0.0656 & 0.1651 & -0.0674 & 0.2506 & 0.0234 & 0.1011 & 0.0193 & -0.0885 & -0.1900 & -0.0909 & 0.0018 \\
\hline 1RQK - BCL L304 & 0.2783 & 0.0448 & 0.0292 & -0.1355 & -0.0876 & -0.0103 & 0.2073 & -0.0862 & 0.2790 & 0.0245 & 0.1465 & 0.0899 & 0.0222 & -0.2029 & 0.0808 & 0.0107 \\
\hline 1RVJ - BCL M854 & 0.2508 & 0.0814 & 0.0905 & -0.0787 & -0.0171 & -0.0168 & 0.2102 & -0.0615 & 0.3183 & 0.0349 & -0.0046 & -0.0235 & -0.2223 & -0.2087 & -0.0319 & -0.0820 \\
\hline 1RY5 - BCL L854 & 0.3785 & 0.0672 & 0.0647 & -0.2050 & -0.0625 & -0.0835 & 0.2774 & -0.0960 & 0.2623 & 0.0243 & -0.0287 & 0.0018 & -0.1907 & -0.1384 & 0.1114 & -0.0095 \\
\hline 1RZH - BCL L854 & 0.3753 & 0.0492 & 0.0944 & -0.2767 & -0.0329 & -0.0126 & 0.2251 & -0.0589 & 0.3962 & 0.0387 & 0.0303 & 0.0441 & -0.1011 & -0.3684 & -0.0662 & -0.0614 \\
\hline 1RZZ - BCL L1004 & 0.2621 & 0.0685 & 0.0300 & -0.1193 & -0.0563 & -0.0504 & 0.1993 & -0.0903 & 0.2664 & 0.0185 & -0.0627 & -0.0291 & -0.1953 & -0.1483 & 0.0401 & -0.0666 \\
\hline $1 S 00$ - BCL L1004 & 0.2741 & 0.0667 & 0.0758 & -0.1518 & -0.0726 & -0.0287 & 0.1778 & -0.0929 & 0.2591 & 0.0189 & 0.0871 & -0.0464 & -0.1766 & -0.1457 & 0.0227 & -0.0668 \\
\hline $1 S 00-B C L$ S2004 & 0.3035 & 0.0664 & 0.0720 & -0.1734 & -0.0240 & 0.0025 & 0.2156 & -0.0991 & 0.4091 & 0.0314 & -0.2209 & -0.0213 & -0.2745 & -0.1534 & 0.1380 & -0.0117 \\
\hline 1UMX - BCL L1283 & 0.4510 & 0.0608 & 0.3753 & 0.0933 & -0.1439 & 0.0032 & 0.1415 & -0.1144 & 0.2370 & 0.0229 & 0.0877 & 0.0451 & -0.0632 & -0.1948 & 0.0599 & -0.0299 \\
\hline 1YF6 - BCL L854 & 0.2429 & 0.0477 & 0.0907 & -0.0873 & -0.0168 & 0.0422 & 0.1724 & -0.1066 & 0.4600 & 0.0557 & 0.1055 & 0.1351 & -0.2827 & -0.3023 & -0.0338 & -0.0986 \\
\hline 1YST - BCL L275 & 0.3032 & 0.0598 & -0.0709 & -0.2094 & 0.0159 & -0.1061 & 0.1446 & -0.1032 & 0.1613 & 0.0113 & -0.0475 & 0.0577 & -0.1209 & -0.0532 & 0.0547 & -0.0029 \\
\hline 1Z9J - BCL B853 & 0.2730 & 0.0605 & -0.1327 & -0.0625 & -0.1771 & 0.0033 & 0.1460 & -0.0191 & 0.0738 & 0.0067 & -0.0041 & 0.0219 & -0.0556 & -0.0280 & 0.0324 & -0.0043 \\
\hline $179 \mathrm{~K}-\mathrm{BCL}$ B853 & 0.2215 & 0.0621 & -0.0789 & -0.0656 & -0.1380 & 0.0729 & 0.1142 & -0.0338 & 0.0888 & 0.0043 & -0.0673 & 0.0221 & -0.0518 & -0.0122 & 0.0062 & 0.0012 \\
\hline 2BNP - BCL A1283 & 0.3664 & 0.0733 & 0.2269 & 0.2039 & -0.1132 & -0.0207 & 0.1006 & -0.1334 & 0.0443 & 0.0145 & 0.0070 & -0.0141 & -0.0139 & -0.0389 & 0.0014 & 0.0017 \\
\hline 2BNS - BCL A1283 & 0.1855 & 0.0685 & -0.0256 & 0.0106 & -0.1096 & 0.0284 & 0.0304 & -0.1411 & 0.0498 & 0.0179 & -0.0048 & 0.0093 & -0.0275 & -0.0361 & -0.0176 & -0.0004 \\
\hline 2BOZ - BCL L1283 & 0.3445 & 0.0497 & -0.0006 & -0.2228 & -0.1121 & -0.0469 & 0.2118 & -0.0968 & 0.4451 & 0.0302 & 0.0695 & 0.0825 & -0.1701 & -0.3503 & -0.1867 & 0.0007 \\
\hline 2GMR - BCL L304 & 0.4409 & 0.0434 & 0.0313 & -0.2069 & -0.0985 & -0.0468 & 0.3663 & -0.0671 & 0.5235 & 0.0498 & 0.0259 & -0.0024 & -0.2649 & -0.4496 & -0.0020 & 0.0329 \\
\hline 2GNU - BCL L1283 & 0.3649 & 0.0630 & 0.1906 & -0.0942 & -0.0630 & -0.0287 & 0.2645 & -0.1149 & 0.1103 & 0.0257 & 0.0217 & 0.0152 & -0.0728 & -0.0638 & -0.0370 & 0.0270 \\
\hline 2HG3 - BCL L314 & 0.3021 & 0.0505 & 0.0699 & -0.1492 & -0.0535 & 0.0040 & 0.2301 & -0.0911 & 0.3375 & 0.0281 & 0.1466 & 0.0139 & -0.0489 & -0.2932 & -0.0621 & -0.0003 \\
\hline 2HG9 - BCL L314 & 0.2843 & 0.0503 & 0.1064 & -0.1050 & -0.1017 & -0.0339 & 0.2032 & -0.0753 & 0.3336 & 0.0346 & 0.0778 & 0.0586 & -0.0571 & -0.3125 & -0.0287 & -0.0056 \\
\hline $2 \mathrm{HH} 1$ - BCL L314 & 0.3409 & 0.0453 & 0.1364 & -0.1345 & -0.0821 & -0.0589 & 0.2506 & -0.0809 & 0.3182 & 0.0304 & 0.1501 & 0.0972 & 0.0143 & -0.2583 & -0.0483 & 0.0003 \\
\hline $2 \mathrm{HHK}-\mathrm{BCL}$ L314 & 0.3012 & 0.0457 & 0.1046 & -0.0783 & -0.0939 & -0.0594 & 0.2223 & -0.1089 & 0.3388 & 0.0287 & 0.1129 & 0.0532 & -0.0446 & -0.3115 & -0.0114 & 0.0055 \\
\hline 2HIT - BCL L314 & 0.3049 & 0.0448 & 0.1006 & -0.1184 & -0.1377 & 0.0244 & 0.1947 & -0.1066 & 0.4170 & 0.0246 & 0.2950 & 0.0534 & 0.0450 & -0.2746 & 0.0810 & 0.0018 \\
\hline 2HJ6 - BCL L314 & 0.2975 & 0.0565 & 0.0110 & -0.2110 & -0.0239 & -0.0024 & 0.1878 & -0.0893 & 0.3737 & 0.0260 & 0.1626 & 0.0516 & -0.0228 & -0.3122 & 0.1116 & -0.0079 \\
\hline $2 \mathrm{~J} 8 \mathrm{C}-\mathrm{BCL}$ L1282 & 0.2781 & 0.0472 & 0.0057 & -0.2001 & -0.1100 & -0.0272 & 0.0939 & -0.1252 & 0.5174 & 0.0638 & -0.0443 & 0.0442 & -0.2034 & -0.4673 & -0.0636 & -0.0043 \\
\hline 2J8D - BCL L1283 & 0.3066 & 0.0468 & -0.0370 & -0.2552 & -0.0249 & -0.0207 & 0.0925 & -0.1338 & 0.4486 & 0.1009 & 0.0359 & 0.0470 & -0.1009 & -0.4066 & -0.1489 & -0.0058 \\
\hline $2 \mathrm{JIY}$ - BCL L1284 & 0.3125 & 0.0432 & 0.0620 & -0.1740 & -0.1168 & -0.0059 & 0.2049 & -0.0886 & 0.3683 & 0.0275 & -0.0679 & 0.0744 & -0.1382 & -0.3224 & -0.0488 & 0.0116 \\
\hline 2JJO - BCL L1284 & 0.2755 & 0.0529 & 0.0108 & -0.1519 & -0.0913 & -0.0693 & 0.1738 & -0.0969 & 0.3215 & 0.0228 & 0.1223 & 0.0721 & -0.0461 & -0.2774 & 0.0614 & 0.0192 \\
\hline 2RCR - BCL L450 & 0.2986 & 0.0501 & 0.1434 & -0.1608 & 0.0869 & -0.0868 & 0.1394 & -0.0907 & 0.3557 & 0.0155 & -0.0686 & 0.1306 & -0.2415 & -0.0096 & 0.2131 & 0.0302 \\
\hline 2UWS - BCL L1282 & 0.2596 & 0.0574 & 0.1174 & -0.1750 & -0.0776 & -0.0175 & -0.0779 & -0.1030 & 0.4339 & 0.1203 & 0.1436 & 0.1193 & -0.1374 & -0.3195 & 0.1728 & 0.0510 \\
\hline 2UWT - BCL L1283 & 0.2612 & 0.0511 & 0.0631 & -0.1935 & -0.1051 & -0.0387 & 0.0276 & -0.1163 & 0.4506 & 0.1023 & 0.1138 & 0.0048 & -0.1507 & -0.4020 & 0.0560 & 0.0511 \\
\hline 2UWU - BCL L1282 & 0.3008 & 0.0420 & 0.0717 & -0.2639 & -0.0464 & -0.0380 & 0.0232 & -0.1075 & 0.4686 & 0.0901 & 0.0857 & 0.0067 & -0.1050 & -0.4480 & -0.0202 & -0.0086 \\
\hline 2UWV - BCL L1282 & 0.2996 & 0.0420 & 0.0688 & -0.2545 & -0.0594 & -0.0126 & 0.0427 & -0.1212 & 0.4425 & 0.0743 & 0.1728 & 0.0182 & -0.1089 & -0.3905 & 0.0249 & -0.0265 \\
\hline 2UWW - BCL L1282 & 0.2780 & 0.0433 & 0.0984 & -0.2315 & -0.0373 & -0.0237 & 0.0211 & -0.1079 & 0.4934 & 0.0919 & 0.1125 & -0.0020 & -0.1076 & -0.4664 & -0.0379 & -0.0161 \\
\hline 2UX3 - BCL L1282 & 0.2680 & 0.0547 & 0.0786 & -0.2030 & -0.0553 & -0.0467 & -0.0267 & -0.1360 & 0.5292 & 0.1024 & 0.0545 & 0.0414 & -0.1873 & -0.4513 & -0.1805 & 0.0635 \\
\hline 2UX4 - BCL L1282 & 0.2308 & 0.0595 & 0.0483 & -0.1832 & -0.0331 & -0.0155 & -0.0298 & -0.1232 & 0.6637 & 0.1103 & 0.0071 & -0.0260 & -0.3127 & -0.5468 & -0.2060 & 0.0247 \\
\hline 2UX5 - BCL L1282 & 0.3327 & 0.0453 & 0.0675 & -0.2903 & -0.0219 & -0.0191 & 0.0884 & -0.1149 & 0.5147 & 0.0791 & 0.0511 & 0.0356 & -0.2114 & -0.4474 & -0.1122 & -0.0599 \\
\hline 2UXJ - BCL L1282 & 0.2783 & 0.0513 & 0.1216 & -0.2115 & -0.0241 & 0.0363 & -0.0485 & -0.1169 & 0.4981 & 0.1074 & 0.1029 & 0.1190 & -0.2495 & -0.3920 & 0.0846 & -0.0160 \\
\hline 2UXK - BCL L1282 & 0.2100 & 0.0536 & 0.0491 & -0.1414 & -0.0448 & -0.0352 & -0.0453 & -0.1280 & 0.5448 & 0.1118 & 0.0591 & 0.0377 & -0.1975 & -0.4694 & -0.1781 & 0.0291 \\
\hline 2UXL - BCL L1282 & 0.2278 & 0.0534 & 0.0598 & -0.1391 & -0.0168 & -0.0147 & -0.0468 & -0.1620 & 0.6437 & 0.1273 & -0.1455 & 0.1012 & -0.2687 & -0.5087 & -0.2248 & 0.0376 \\
\hline 2UXM - BCL L1283 & 0.2853 & 0.0594 & 0.0489 & -0.2426 & -0.0351 & 0.0339 & -0.0236 & -0.1312 & 0.5963 & 0.1244 & 0.0640 & -0.0853 & -0.2845 & -0.4641 & -0.2173 & -0.0247 \\
\hline
\end{tabular}




\begin{tabular}{|c|c|c|c|c|c|c|c|c|c|c|c|c|c|c|c|c|}
\hline Cofactor ID & Dip & Sip & B2g & B1g & $\mathrm{Eu}(\mathrm{x})$ & $\mathrm{Eu}(\mathrm{y})$ & A1g & $\mathrm{A} 2 \mathrm{~g}$ & Doop & Soop & $\mathrm{B} 2 \mathrm{u}$ & B1u & $A 2 u$ & $\mathrm{Eg}(\mathrm{x})$ & $\mathrm{Eg}(\mathrm{y})$ & A1u \\
\hline 2WX5 - BCL M1304 & 0.2572 & 0.0554 & 0.1051 & -0.0220 & -0.1907 & 0.0361 & -0.0890 & -0.0948 & 0.2858 & 0.0522 & 0.0477 & 0.0215 & -0.1012 & -0.2599 & -0.0098 & -0.0327 \\
\hline 3DSY - BCL L501 & 0.3536 & 0.0509 & -0.0139 & -0.2129 & -0.1210 & 0.0218 & 0.2456 & -0.0639 & 0.3998 & 0.0676 & -0.0826 & 0.0070 & -0.2593 & -0.1960 & -0.2126 & 0.0456 \\
\hline 3DTA - BCL L501 & 0.3540 & 0.0509 & -0.0142 & -0.2129 & -0.1210 & 0.0222 & 0.2461 & -0.0642 & 0.3996 & 0.0677 & -0.0824 & 0.0063 & -0.2595 & -0.1956 & -0.2124 & 0.0458 \\
\hline 3DTR - BCL L501 & 0.3541 & 0.0509 & -0.0142 & -0.2130 & -0.1210 & 0.0219 & 0.2460 & -0.0645 & 0.3993 & 0.0676 & -0.0822 & 0.0068 & -0.2590 & -0.1963 & -0.2121 & 0.0450 \\
\hline 3DTS - BCL L501 & 0.3546 & 0.0509 & -0.0144 & -0.2135 & -0.1200 & 0.0219 & 0.2471 & -0.0631 & 0.3996 & 0.0677 & -0.0828 & 0.0068 & -0.2593 & -0.1953 & -0.2128 & 0.0458 \\
\hline 3DU2 - BCL L501 & 0.3537 & 0.0510 & -0.0142 & -0.2130 & -0.1206 & 0.0216 & 0.2458 & -0.0638 & 0.4000 & 0.0677 & -0.0827 & 0.0059 & -0.2595 & -0.1958 & -0.2129 & 0.0463 \\
\hline 3DU3 - BCL L501 & 0.3548 & 0.0510 & -0.0150 & -0.2135 & -0.1204 & 0.0212 & 0.2471 & -0.0636 & 0.3995 & 0.0679 & -0.0821 & 0.0064 & -0.2592 & -0.1953 & -0.2131 & 0.0460 \\
\hline 3DUQ - BCL L501 & 0.3549 & 0.0509 & -0.0148 & -0.2134 & -0.1209 & 0.0219 & 0.2470 & -0.0637 & 0.3992 & 0.0676 & -0.0822 & 0.0075 & -0.2590 & -0.1950 & -0.2130 & 0.0460 \\
\hline 4RCR - BCL M311 & 0.5346 & 0.0592 & 0.0850 & -0.0551 & 0.2437 & -0.0723 & 0.4591 & 0.0112 & 0.5349 & 0.0596 & 0.4237 & 0.0466 & -0.0377 & -0.1265 & 0.2615 & 0.1366 \\
\hline
\end{tabular}

Table 48 : Minimum basis normal-mode displacements and conformational parameters of all BB cofactors from crystal structures of Rhodobacter sphaeroides.

\begin{tabular}{|c|c|c|c|c|c|c|c|c|c|c|c|c|c|c|c|c|}
\hline Cofactor ID & Dip & $\delta$ ip & B2g & B1g & $\mathrm{Eu}(\mathrm{x})$ & $\mathrm{Eu}(\mathrm{y})$ & A1g & $A 2 g$ & Doop & Soop & $\mathrm{B} 2 \mathrm{u}$ & B1u & $A 2 u$ & $\mathrm{Eg}(\mathrm{x})$ & $\mathrm{Eg}(\mathrm{y})$ & A1u \\
\hline 1AIG - BCL M309 & 0.2535 & 0.0528 & -0.1265 & -0.0414 & -0.0650 & -0.1063 & 0.1652 & -0.0609 & 0.2137 & 0.0103 & 0.0670 & 0.1026 & -0.0893 & -0.1461 & -0.0360 & 0.0071 \\
\hline 1AIG - BCL N282 & 0.3205 & 0.0522 & -0.0282 & -0.0834 & -0.0728 & -0.0747 & 0.2881 & -0.0328 & 0.3919 & 0.0155 & 0.0863 & 0.3518 & -0.1160 & -0.0765 & 0.0445 & 0.0325 \\
\hline 1AIJ - BCL M309 & 0.2613 & 0.0528 & -0.0120 & -0.0886 & -0.0324 & -0.0684 & 0.2272 & -0.0541 & 0.4255 & 0.0148 & 0.2147 & 0.2992 & -0.1330 & -0.1522 & 0.0626 & 0.0257 \\
\hline 1AIJ - BCL S309 & 0.2824 & 0.0527 & -0.0075 & -0.0826 & -0.0302 & -0.0870 & 0.2488 & -0.0499 & 0.3895 & 0.0107 & 0.1001 & 0.2955 & -0.1664 & -0.1538 & 0.0412 & 0.0363 \\
\hline 1DS8 - BCL L1001 & 0.2808 & 0.0562 & -0.1079 & -0.0859 & -0.0718 & -0.0520 & 0.2107 & -0.0872 & 0.5191 & 0.0261 & 0.1839 & 0.3608 & -0.1362 & -0.2837 & 0.0488 & 0.0642 \\
\hline 1DS8 - BCL R2001 & 0.3153 & 0.0561 & 0.0273 & -0.0928 & 0.0397 & -0.1206 & 0.2656 & -0.0582 & 0.6416 & 0.0195 & 0.2234 & 0.5110 & -0.1836 & -0.2347 & 0.0376 & 0.1017 \\
\hline 1DV3 - BCL L1001 & 0.2850 & 0.0571 & -0.0097 & -0.0975 & -0.0383 & -0.0929 & 0.2334 & -0.0838 & 0.5403 & 0.0234 & 0.2381 & 0.3578 & -0.1330 & -0.2632 & 0.1364 & 0.0393 \\
\hline 1DV3 - BCL S2001 & 0.3816 & 0.0598 & 0.0394 & -0.1468 & 0.0274 & -0.0826 & 0.3362 & -0.0437 & 0.6704 & 0.0198 & 0.1106 & 0.5845 & -0.1899 & -0.2139 & 0.0660 & 0.0974 \\
\hline 1DV6 - BCL M1001 & 0.3066 & 0.0554 & -0.0667 & -0.1097 & -0.0134 & -0.0646 & 0.2580 & -0.0813 & 0.5867 & 0.0253 & 0.2313 & 0.4361 & -0.1217 & -0.2677 & 0.1032 & 0.0590 \\
\hline 1DV6 - BCL S2001 & 0.3454 & 0.0599 & -0.0360 & -0.1250 & 0.0345 & -0.0662 & 0.3065 & -0.0534 & 0.6357 & 0.0161 & 0.0842 & 0.5298 & -0.2329 & -0.2397 & 0.0188 & 0.0652 \\
\hline $1 \mathrm{E} 14$ - BCL C301 & 0.4279 & 0.0575 & 0.1211 & -0.2730 & -0.0862 & -0.1041 & 0.2669 & -0.0664 & 0.7275 & 0.0240 & 0.1794 & 0.5817 & 0.0245 & -0.3362 & 0.2069 & 0.0474 \\
\hline 1E6D - BCL C301 & 0.3809 & 0.0549 & 0.1028 & -0.2285 & -0.0795 & -0.0735 & 0.2610 & -0.0500 & 0.7048 & 0.0183 & 0.2315 & 0.5498 & -0.0038 & -0.3019 & 0.2206 & 0.0325 \\
\hline 1EYS - BCL M601 & 0.3635 & 0.0469 & -0.0262 & -0.1883 & -0.1691 & -0.1448 & 0.1887 & -0.1043 & 0.4407 & 0.0796 & -0.1807 & 0.2928 & 0.0383 & -0.1586 & 0.2088 & 0.0751 \\
\hline $1 F 6 N-B C L$ L301 & 0.4289 & 0.0398 & 0.0912 & -0.2319 & -0.0378 & -0.0720 & 0.3328 & -0.0673 & 0.6966 & 0.0678 & 0.2936 & 0.4649 & -0.3115 & -0.2838 & 0.0727 & -0.0023 \\
\hline 1FNP - BCL M801 & 0.4687 & 0.0426 & 0.0939 & -0.2842 & -0.0560 & -0.0861 & 0.3409 & -0.0580 & 0.6014 & 0.0562 & 0.2617 & 0.4019 & -0.2005 & -0.2423 & 0.1810 & -0.0080 \\
\hline 1FNQ - BCL M801 & 0.4188 & 0.0393 & 0.0736 & -0.2464 & -0.0320 & -0.0993 & 0.3062 & -0.0677 & 0.6395 & 0.0669 & 0.3098 & 0.3877 & -0.1787 & -0.3498 & 0.0724 & -0.0563 \\
\hline 1JGW - BCL L850 & 0.3482 & 0.0594 & -0.0687 & -0.0741 & -0.1192 & -0.0521 & 0.3051 & -0.0313 & 0.1296 & 0.0056 & 0.0333 & 0.0908 & -0.0743 & -0.0350 & 0.0262 & 0.0029 \\
\hline 1JGX - BCL L850 & 0.3107 & 0.0917 & -0.1649 & 0.0025 & -0.0529 & 0.0279 & 0.2562 & -0.0118 & 0.3260 & 0.0286 & -0.2679 & -0.0567 & -0.1195 & 0.0213 & -0.0612 & 0.1133 \\
\hline 1JGY - BCL L850 & 0.3850 & 0.0574 & -0.0802 & -0.0728 & -0.1733 & -0.0626 & 0.3182 & -0.0359 & 0.1066 & 0.0048 & 0.0465 & 0.0469 & -0.0759 & -0.0238 & 0.0258 & 0.0029 \\
\hline 1JGZ - BCL L850 & 0.4048 & 0.0604 & -0.1196 & -0.0294 & -0.0814 & -0.0703 & 0.3693 & -0.0267 & 0.1237 & 0.0046 & 0.0713 & 0.0603 & -0.0760 & -0.0249 & 0.0134 & 0.0015 \\
\hline 1JHO - BCL M850 & 0.3492 & 0.0953 & -0.1982 & 0.0261 & -0.1148 & 0.0850 & 0.2480 & -0.0056 & 0.3177 & 0.0293 & -0.2561 & -0.0753 & -0.1120 & 0.0361 & -0.0686 & 0.1054 \\
\hline 1K6L - BCL M501 & 0.3999 & 0.0510 & 0.0561 & -0.2651 & -0.0414 & -0.0496 & 0.2767 & -0.0754 & 0.7070 & 0.0167 & 0.2957 & 0.5347 & 0.0763 & -0.3020 & 0.1696 & 0.0277 \\
\hline 1K6N - BCL M501 & 0.3996 & 0.0509 & 0.0563 & -0.2649 & -0.0417 & -0.0503 & 0.2766 & -0.0745 & 0.7071 & 0.0167 & 0.2956 & 0.5347 & 0.0757 & -0.3022 & 0.1696 & 0.0274 \\
\hline $1 \mathrm{KBY}-\mathrm{BCL}$ L850 & 0.3286 & 0.0925 & -0.1424 & 0.0106 & -0.0620 & 0.0708 & 0.2806 & -0.0056 & 0.3002 & 0.0275 & -0.2372 & -0.0477 & -0.1223 & 0.0351 & -0.0659 & 0.1052 \\
\hline 1L9B - BCL M1001 & 0.3114 & 0.0694 & 0.0718 & -0.1698 & -0.0565 & 0.0134 & 0.2217 & -0.1023 & 0.2383 & 0.0143 & 0.0696 & 0.0403 & -0.1768 & -0.0898 & 0.1049 & -0.0021 \\
\hline 1L9J - BCL M1001 & 0.3050 & 0.0684 & 0.0555 & -0.1579 & -0.0417 & -0.0914 & 0.2133 & -0.0971 & 0.2716 & 0.0220 & 0.0612 & 0.0485 & -0.1965 & -0.0938 & 0.1415 & 0.0154 \\
\hline 1L9J - BCL S2001 & 0.3264 & 0.0636 & 0.0857 & -0.2270 & -0.0392 & -0.1140 & 0.1453 & -0.1095 & 0.2835 & 0.0209 & -0.1015 & 0.0250 & -0.2133 & -0.1180 & 0.0936 & -0.0354 \\
\hline 1M3X - BCL L850 & 0.3535 & 0.0588 & -0.0751 & -0.0505 & -0.1391 & -0.0487 & 0.3065 & -0.0332 & 0.1489 & 0.0052 & 0.0797 & 0.0869 & -0.0797 & -0.0380 & 0.0216 & -0.0031 \\
\hline 1MPS - BCL M801 & 0.4539 & 0.0435 & 0.0685 & -0.2337 & -0.0614 & -0.1079 & 0.3571 & -0.0613 & 0.4879 & 0.0453 & 0.1566 & 0.2909 & -0.1276 & -0.3097 & 0.1263 & 0.0282 \\
\hline
\end{tabular}




\begin{tabular}{|c|c|c|c|c|c|c|c|c|c|c|c|c|c|c|c|c|}
\hline Cofactor ID & Dip & Sip & B2g & B1g & $\mathrm{Eu}(\mathrm{x})$ & $\mathrm{Eu}(\mathrm{y})$ & A1g & $\mathrm{A} 2 \mathrm{~g}$ & Doop & Soop & $\mathrm{B} 2 \mathrm{u}$ & B1u & $A 2 u$ & $\mathrm{Eg}(\mathrm{x})$ & $\mathrm{Eg}(\mathrm{y})$ & A1u \\
\hline 10GV - BCL M1302 & 0.3841 & 0.0657 & 0.0604 & -0.2366 & -0.0764 & -0.0613 & 0.2641 & -0.0926 & 0.2882 & 0.0182 & -0.1606 & 0.0884 & -0.1861 & -0.0831 & 0.0883 & 0.0117 \\
\hline 1PCR - BCL L301 & 0.4079 & 0.0427 & 0.0831 & -0.3037 & -0.0868 & -0.1492 & 0.1850 & -0.0567 & 0.6496 & 0.0666 & 0.2258 & 0.5127 & -0.1166 & -0.2878 & 0.1072 & -0.0151 \\
\hline 1PSS - BCL M1 & 0.3011 & 0.0498 & -0.0452 & -0.1467 & -0.0491 & -0.1044 & 0.2265 & -0.0497 & 0.2196 & 0.0053 & -0.0060 & 0.2016 & -0.0825 & -0.0158 & -0.0071 & 0.0206 \\
\hline 1PST - BCL M1 & 0.2866 & 0.0510 & -0.0482 & -0.0650 & -0.0095 & -0.1400 & 0.2313 & -0.0488 & 0.3476 & 0.0124 & 0.1133 & 0.2480 & -0.1944 & -0.0810 & 0.0388 & 0.0249 \\
\hline 1PYH - BCL A301 & 0.3851 & 0.0495 & 0.0445 & -0.3138 & -0.0295 & -0.1179 & 0.1610 & -0.0850 & 0.6579 & 0.0217 & 0.2545 & 0.5034 & 0.0455 & -0.2947 & 0.1489 & 0.0597 \\
\hline 1QOV - BCL M1301 & 0.3990 & 0.0509 & 0.0560 & -0.2647 & -0.0402 & -0.0503 & 0.2760 & -0.0752 & 0.7064 & 0.0166 & 0.2950 & 0.5341 & 0.0772 & -0.3021 & 0.1694 & 0.0278 \\
\hline 1RG5 - BCL M501 & 0.5280 & 0.0546 & -0.0171 & -0.3444 & -0.0438 & -0.1158 & 0.3699 & -0.0878 & 0.4964 & 0.0179 & 0.2581 & 0.2676 & -0.0089 & -0.2889 & 0.1438 & 0.0634 \\
\hline 1RGN - BCL M908 & 0.4439 & 0.0574 & 0.0959 & -0.2912 & -0.0658 & -0.1830 & 0.2438 & -0.0761 & 0.7149 & 0.0270 & 0.1433 & 0.5595 & -0.0290 & -0.2404 & 0.3355 & 0.0799 \\
\hline 1RQK - BCL M501 & 0.4305 & 0.0576 & -0.1019 & -0.3233 & 0.0479 & -0.1258 & 0.2160 & -0.0753 & 0.6429 & 0.0192 & 0.3049 & 0.3734 & -0.0288 & -0.3181 & 0.2759 & 0.0535 \\
\hline 1RVJ - BCL L851 & 0.2970 & 0.0696 & 0.0794 & -0.1334 & -0.0307 & -0.0608 & 0.2241 & -0.0959 & 0.3694 & 0.0266 & 0.0322 & 0.1921 & -0.2119 & -0.1798 & 0.1452 & 0.0127 \\
\hline 1RY5 - BCL L851 & 0.3118 & 0.0702 & 0.0846 & -0.1528 & 0.0684 & -0.0996 & 0.2130 & -0.0822 & 0.4116 & 0.0347 & -0.0918 & 0.0967 & -0.2908 & -0.2446 & 0.0627 & -0.0575 \\
\hline 1RZH - BCL M851 & 0.3985 & 0.0508 & 0.0557 & -0.2646 & -0.0401 & -0.0503 & 0.2756 & -0.0750 & 0.7059 & 0.0167 & 0.2942 & 0.5342 & 0.0772 & -0.3019 & 0.1688 & 0.0281 \\
\hline 1RZZ - BCL L1001 & 0.2524 & 0.0691 & 0.1004 & -0.1348 & -0.0276 & -0.0228 & 0.1522 & -0.1048 & 0.3487 & 0.0252 & 0.0264 & 0.1112 & -0.2655 & -0.1474 & 0.1275 & -0.0105 \\
\hline 1RZZ - BCL S2001 & 0.3075 & 0.0686 & 0.0728 & -0.1552 & -0.0205 & -0.0814 & 0.2195 & -0.0997 & 0.3211 & 0.0218 & 0.1048 & 0.1125 & -0.2132 & -0.1122 & 0.1441 & 0.0254 \\
\hline 1RZZ - BCL S2004 & 0.3033 & 0.0659 & 0.0779 & -0.1642 & -0.0276 & -0.0119 & 0.2200 & -0.0983 & 0.3946 & 0.0326 & -0.1689 & -0.0169 & -0.2809 & -0.1698 & 0.1372 & -0.0192 \\
\hline 1S00 - BCL L1001 & 0.2212 & 0.0683 & 0.0921 & -0.1325 & -0.0097 & -0.0239 & 0.1129 & -0.0975 & 0.3834 & 0.0283 & -0.0016 & 0.1428 & -0.2870 & -0.1584 & 0.1381 & -0.0070 \\
\hline $1 \mathrm{~S} 00$ - BCL S2001 & 0.2858 & 0.0706 & 0.0501 & -0.1520 & -0.0273 & -0.0988 & 0.1897 & -0.0980 & 0.2427 & 0.0156 & 0.0671 & 0.0793 & -0.1713 & -0.1048 & 0.0877 & -0.0076 \\
\hline $1 U M X-B C L$ M1303 & 0.4439 & 0.0499 & 0.0752 & -0.3831 & -0.0014 & -0.1558 & 0.1051 & -0.0966 & 0.6360 & 0.0193 & 0.2411 & 0.4211 & -0.0617 & -0.2715 & 0.3014 & 0.0271 \\
\hline 1YF6 - BCL M851 & 0.2939 & 0.0574 & 0.0200 & -0.2547 & -0.0667 & 0.0226 & 0.0592 & -0.1122 & 0.7856 & 0.0500 & 0.4103 & 0.5667 & -0.0063 & -0.3300 & 0.0767 & 0.1133 \\
\hline 1YST - BCL M307 & 0.4590 & 0.0825 & 0.2431 & -0.3530 & 0.1105 & -0.0305 & 0.0719 & -0.0930 & 0.1919 & 0.0194 & -0.1126 & 0.1104 & -0.0344 & -0.0912 & -0.0201 & 0.0454 \\
\hline 1Z9J - BCL A850 & 0.3415 & 0.0596 & -0.0945 & -0.0961 & -0.2532 & -0.0022 & 0.1845 & -0.0171 & 0.1739 & 0.0055 & -0.1710 & -0.0025 & 0.0145 & 0.0198 & 0.0111 & 0.0164 \\
\hline $1 Z 9 K-B C L A 850$ & 0.4561 & 0.0635 & -0.2067 & -0.0408 & -0.2765 & -0.0285 & 0.2922 & -0.0316 & 0.1545 & 0.0044 & -0.1255 & 0.0777 & -0.0201 & -0.0258 & 0.0315 & 0.0029 \\
\hline 2BNP - BCL B1302 & 0.4237 & 0.0648 & 0.3391 & -0.1075 & -0.1815 & 0.0120 & 0.0629 & -0.1261 & 0.0588 & 0.0126 & -0.0114 & 0.0450 & -0.0153 & -0.0246 & 0.0116 & 0.0179 \\
\hline 2BNS - BCL B1302 & 0.3922 & 0.0636 & 0.2442 & -0.2180 & -0.1424 & 0.0404 & 0.0968 & -0.1241 & 0.0463 & 0.0136 & -0.0187 & 0.0262 & -0.0217 & -0.0131 & 0.0029 & 0.0213 \\
\hline 2BOZ - BCL M1303 & 0.3776 & 0.0503 & 0.0667 & -0.2654 & -0.0702 & -0.1660 & 0.1582 & -0.1007 & 0.7540 & 0.0196 & 0.1165 & 0.6149 & 0.0010 & -0.3593 & 0.2145 & 0.0404 \\
\hline 2GMR - BCL L301 & 0.4544 & 0.0438 & 0.1092 & -0.2831 & -0.0551 & -0.1281 & 0.2995 & -0.0721 & 0.7773 & 0.0448 & 0.2214 & 0.6166 & -0.1716 & -0.3228 & 0.1622 & 0.1222 \\
\hline 2GNU - BCL M1302 & 0.3649 & 0.0630 & 0.1906 & -0.0942 & -0.0630 & -0.0287 & 0.2645 & -0.1149 & 0.1103 & 0.0257 & 0.0217 & 0.0152 & -0.0728 & -0.0638 & -0.0370 & 0.0270 \\
\hline 2HG3 - BCL M311 & 0.4036 & 0.0540 & 0.1315 & -0.2693 & -0.0438 & -0.1211 & 0.2267 & -0.0714 & 0.4403 & 0.0204 & 0.0892 & 0.2861 & 0.0907 & -0.2943 & 0.0672 & 0.0686 \\
\hline 2HG9 - BCL M311 & 0.3912 & 0.0534 & 0.0238 & -0.3093 & -0.0059 & -0.1077 & 0.1780 & -0.1161 & 0.5694 & 0.0210 & 0.1874 & 0.3904 & 0.0488 & -0.3236 & 0.1592 & 0.0653 \\
\hline $2 \mathrm{HH} 1-\mathrm{BCL}$ M311 & 0.4494 & 0.0532 & 0.0720 & -0.3404 & -0.0673 & -0.0900 & 0.2469 & -0.0854 & 0.5193 & 0.0203 & 0.1546 & 0.3240 & 0.0351 & -0.3423 & 0.1388 & 0.0565 \\
\hline $2 \mathrm{HHK}-\mathrm{BCL}$ M311 & 0.4506 & 0.0481 & 0.0570 & -0.3124 & -0.0779 & -0.0528 & 0.2932 & -0.0859 & 0.4986 & 0.0216 & 0.2002 & 0.2789 & 0.0829 & -0.3291 & 0.1154 & 0.0474 \\
\hline 2HIT - BCL M311 & 0.3859 & 0.0458 & 0.1079 & -0.2379 & -0.1309 & -0.0937 & 0.2152 & -0.0921 & 0.5316 & 0.0217 & 0.2172 & 0.3790 & 0.0374 & -0.2604 & 0.1390 & 0.0577 \\
\hline 2HJ6 - BCL M311 & 0.4560 & 0.0492 & 0.0999 & -0.3601 & -0.1317 & -0.1672 & 0.1154 & -0.0981 & 0.5971 & 0.0216 & 0.1591 & 0.4633 & -0.0424 & -0.3137 & 0.1134 & 0.0590 \\
\hline 2J8C - BCL M1303 & 0.2969 & 0.0474 & -0.0200 & -0.2141 & -0.0504 & -0.1305 & 0.0854 & -0.1228 & 0.7657 & 0.0584 & 0.2673 & 0.5909 & -0.0086 & -0.3939 & 0.0691 & 0.0755 \\
\hline 2J8D - BCL M1308 & 0.3057 & 0.0487 & 0.1381 & -0.2227 & -0.0429 & -0.0697 & -0.0467 & -0.1262 & 0.7106 & 0.0648 & 0.3075 & 0.5430 & -0.0154 & -0.3092 & 0.1364 & 0.0320 \\
\hline $2 J I Y-B C L ~ L 1282$ & 0.4434 & 0.0524 & -0.0007 & -0.3531 & -0.0431 & -0.0950 & 0.2343 & -0.0783 & 0.6573 & 0.0234 & 0.2707 & 0.4388 & -0.0699 & -0.3739 & 0.1417 & 0.0384 \\
\hline 2JJO - BCL L1282 & 0.4152 & 0.0539 & 0.0446 & -0.2935 & -0.0889 & -0.1727 & 0.2000 & -0.0809 & 0.6402 & 0.0256 & 0.1504 & 0.4684 & -0.0309 & -0.3327 & 0.2319 & 0.0496 \\
\hline 2RCR - BCL M601 & 0.4219 & 0.0490 & 0.1877 & -0.2922 & 0.0016 & -0.0743 & 0.2153 & -0.0745 & 0.4668 & 0.0122 & 0.0730 & -0.3431 & -0.2001 & 0.0942 & 0.2142 & 0.0083 \\
\hline 2UWS - BCL M1303 & 0.3906 & 0.0600 & 0.1873 & -0.2200 & -0.0180 & -0.1891 & -0.1053 & -0.1481 & 0.8644 & 0.1258 & 0.2499 & 0.5925 & -0.2174 & -0.4535 & 0.0331 & 0.2822 \\
\hline 2UWT - BCL M1303 & 0.3370 & 0.0514 & 0.1077 & -0.2356 & 0.0213 & -0.1263 & -0.0902 & -0.1481 & 0.6665 & 0.0831 & 0.2573 & 0.5176 & -0.0420 & -0.2500 & 0.1619 & 0.1402 \\
\hline 2UWU - BCL M1303 & 0.2621 & 0.0515 & 0.0063 & -0.2045 & 0.0117 & -0.1073 & -0.0296 & -0.1196 & 0.6749 & 0.0613 & 0.2918 & 0.4875 & -0.0290 & -0.3242 & 0.1623 & 0.0196 \\
\hline 2UWV - BCL M1303 & 0.3006 & 0.0463 & 0.0614 & -0.2132 & 0.0371 & -0.1351 & -0.0927 & -0.1136 & 0.6852 & 0.0520 & 0.2719 & 0.4911 & -0.0183 & -0.3548 & 0.1670 & 0.0176 \\
\hline 2UWW - BCL M1304 & 0.2847 & 0.0534 & 0.0271 & -0.2405 & 0.0095 & -0.0962 & -0.0145 & -0.1136 & 0.7212 & 0.0617 & 0.3027 & 0.5324 & -0.0363 & -0.3406 & 0.1489 & 0.0738 \\
\hline
\end{tabular}




\begin{tabular}{|c|c|c|c|c|c|c|c|c|c|c|c|c|c|c|c|c|}
\hline Cofactor ID & Dip & $\delta$ ip & B2g & B1g & $\mathrm{Eu}(\mathrm{x})$ & Eu(y) & A1g & $A 2 g$ & Doop & Soop & $\mathrm{B} 2 \mathrm{u}$ & B1u & $\mathrm{A} 2 \mathrm{u}$ & $\mathrm{Eg}(\mathrm{x})$ & $\mathrm{Eg}(\mathrm{y})$ & A1u \\
\hline $2 U X 3-B C L$ M1303 & 0.3460 & 0.0579 & 0.1588 & -0.2301 & 0.0587 & -0.1042 & -0.0865 & -0.1406 & 0.7221 & 0.0924 & 0.3256 & 0.4942 & -0.0176 & -0.3762 & 0.0888 & 0.1465 \\
\hline $2 U X 4$ - BCL M1303 & 0.2855 & 0.0539 & 0.0605 & -0.1882 & 0.0130 & -0.1451 & -0.0786 & -0.1225 & 0.7575 & 0.0916 & 0.2903 & 0.5850 & -0.0347 & -0.3129 & 0.2054 & 0.0776 \\
\hline 2UX5 - BCL M1304 & 0.2849 & 0.0480 & 0.0952 & -0.1696 & 0.0290 & -0.0836 & -0.1515 & -0.1120 & 0.6859 & 0.0576 & 0.2777 & 0.4723 & -0.0301 & -0.3674 & 0.1848 & 0.0144 \\
\hline $2 U X J-B C L M 1303$ & 0.3096 & 0.0464 & 0.0674 & -0.2379 & -0.0573 & -0.0497 & -0.0753 & -0.1525 & 0.8292 & 0.0655 & 0.2615 & 0.6530 & -0.0934 & -0.4142 & 0.0790 & 0.0787 \\
\hline 2UXK - BCL M1303 & 0.3165 & 0.0557 & 0.1401 & -0.1888 & -0.0463 & -0.1577 & -0.0644 & -0.1173 & 0.9027 & 0.0846 & 0.2629 & 0.7494 & -0.0236 & -0.3620 & 0.1399 & 0.1818 \\
\hline 2UXL - BCL M1303 & 0.3512 & 0.0578 & 0.1555 & -0.2449 & -0.0832 & -0.0435 & -0.0932 & -0.1472 & 0.7851 & 0.1044 & -0.1329 & 0.3059 & -0.3823 & -0.5951 & 0.0153 & 0.0666 \\
\hline $2 U X M-B C L ~ M 1303$ & 0.3495 & 0.0626 & 0.2045 & -0.1905 & -0.0233 & -0.1364 & -0.1063 & -0.1167 & 0.7930 & 0.0892 & 0.2749 & 0.5599 & -0.0521 & -0.3850 & 0.2199 & 0.2012 \\
\hline $2 W X 5$ - BCL L1282 & 0.2332 & 0.0465 & -0.0241 & -0.1037 & 0.0237 & -0.1547 & -0.1026 & -0.0897 & 0.6298 & 0.0364 & 0.0581 & 0.4958 & -0.0684 & -0.2113 & 0.2675 & 0.1631 \\
\hline 3DSY - BCL M501 & 0.3998 & 0.0509 & 0.0562 & -0.2653 & -0.0412 & -0.0501 & 0.2765 & -0.0752 & 0.7070 & 0.0167 & 0.2953 & 0.5349 & 0.0764 & -0.3017 & 0.1697 & 0.0276 \\
\hline 3DTA - BCL M501 & 0.3998 & 0.0509 & 0.0560 & -0.2648 & -0.0414 & -0.0496 & 0.2770 & -0.0753 & 0.7072 & 0.0167 & 0.2959 & 0.5347 & 0.0764 & -0.3018 & 0.1701 & 0.0276 \\
\hline 3DTR - BCL M501 & 0.3999 & 0.0509 & 0.0562 & -0.2654 & -0.0410 & -0.0493 & 0.2767 & -0.0752 & 0.7070 & 0.0167 & 0.2954 & 0.5347 & 0.0767 & -0.3019 & 0.1697 & 0.0280 \\
\hline 3DTS - BCL M501 & 0.3993 & 0.0510 & 0.0560 & -0.2652 & -0.0417 & -0.0504 & 0.2761 & -0.0740 & 0.7069 & 0.0168 & 0.2954 & 0.5346 & 0.0758 & -0.3025 & 0.1696 & 0.0275 \\
\hline 3DU2 - BCL M501 & 0.3998 & 0.0509 & 0.0563 & -0.2653 & -0.0413 & -0.0499 & 0.2764 & -0.0754 & 0.7069 & 0.0167 & 0.2954 & 0.5345 & 0.0760 & -0.3024 & 0.1697 & 0.0277 \\
\hline 3DU3 - BCL M501 & 0.4002 & 0.0509 & 0.0563 & -0.2652 & -0.0419 & -0.0505 & 0.2770 & -0.0752 & 0.7077 & 0.0167 & 0.2965 & 0.5348 & 0.0759 & -0.3020 & 0.1706 & 0.0280 \\
\hline 3DUQ - BCL M501 & 0.3993 & 0.0511 & 0.0568 & -0.2651 & -0.0410 & -0.0501 & 0.2761 & -0.0743 & 0.7063 & 0.0167 & 0.2951 & 0.5341 & 0.0755 & -0.3023 & 0.1695 & 0.0278 \\
\hline 4RCR - BCL L282 & 0.4363 & 0.0352 & -0.0276 & -0.0790 & -0.0280 & -0.0289 & 0.4261 & 0.0124 & 0.5507 & 0.0679 & 0.0452 & 0.1944 & -0.4909 & -0.1241 & -0.0534 & 0.0645 \\
\hline
\end{tabular}

Table 49: Minimum basis normal-mode displacements and conformational parameters of all DL cofactors from crystal structures of Rhodobacter sphaeroides.

\begin{tabular}{|c|c|c|c|c|c|c|c|c|c|c|c|c|c|c|c|c|}
\hline Cofactor ID & Dip & Sip & B2g & B1g & $\mathrm{Eu}(\mathrm{x})$ & $\mathrm{Eu}(\mathrm{y})$ & A1g & $\mathrm{A} 2 \mathrm{~g}$ & Doop & Soop & B2u & B1u & $\mathrm{A} 2 \mathrm{u}$ & $\mathrm{Eg}(\mathrm{x})$ & $\mathrm{Eg}(\mathrm{y})$ & A1u \\
\hline 1AIG - BCL L282 & 0.3228 & 0.0501 & -0.1203 & -0.1097 & -0.0909 & -0.0711 & 0.2463 & -0.0611 & 0.3885 & 0.0137 & 0.1476 & 0.2466 & -0.2148 & -0.1123 & -0.0895 & 0.0398 \\
\hline 1AIG - BCL N283 & 0.3080 & 0.0473 & -0.1176 & -0.0447 & -0.0518 & -0.0563 & 0.2646 & -0.0565 & 0.5026 & 0.0142 & 0.2475 & 0.3314 & -0.2642 & -0.0376 & -0.0943 & 0.0374 \\
\hline 1AIJ - BCL L282 & 0.3189 & 0.0514 & -0.1060 & -0.0897 & -0.0671 & -0.0765 & 0.2615 & -0.0607 & 0.5339 & 0.0178 & 0.2361 & 0.3183 & -0.3421 & -0.0713 & -0.0669 & 0.0379 \\
\hline 1AIJ - BCL R282 & 0.3119 & 0.0524 & -0.0853 & -0.0715 & -0.0553 & -0.0694 & 0.2706 & -0.0615 & 0.4597 & 0.0138 & 0.1835 & 0.2907 & -0.2933 & -0.0633 & -0.0498 & 0.0261 \\
\hline 1DS8 - BCL L1002 & 0.3526 & 0.0500 & -0.0885 & -0.1272 & -0.0370 & -0.0812 & 0.2972 & -0.0634 & 0.5818 & 0.0236 & 0.2350 & 0.2720 & -0.4176 & -0.1203 & -0.1254 & 0.0690 \\
\hline 1DS8 - BCL R2002 & 0.3831 & 0.0524 & -0.1432 & -0.0295 & -0.0248 & -0.0475 & 0.3419 & -0.0747 & 0.5400 & 0.0270 & 0.2527 & 0.1746 & -0.4259 & -0.1029 & -0.0544 & 0.0475 \\
\hline 1DV3 - BCL L1002 & 0.4262 & 0.0498 & -0.1481 & -0.1424 & -0.0915 & -0.0570 & 0.3520 & -0.0624 & 0.5381 & 0.0223 & 0.2591 & 0.2146 & -0.3968 & -0.1155 & -0.0493 & 0.0556 \\
\hline 1DV3 - BCL R2002 & 0.4262 & 0.0543 & -0.1693 & -0.1426 & -0.0495 & -0.0606 & 0.3515 & -0.0543 & 0.5753 & 0.0239 & 0.3118 & 0.2079 & -0.4185 & -0.0931 & -0.0682 & 0.0458 \\
\hline 1DV6 - BCL L1002 & 0.3918 & 0.0488 & -0.1278 & -0.1336 & -0.0627 & -0.0908 & 0.3218 & -0.0597 & 0.6248 & 0.0320 & 0.3377 & 0.2801 & -0.4267 & -0.0908 & -0.0663 & 0.0560 \\
\hline 1DV6 - BCL R2002 & 0.4355 & 0.0542 & -0.1988 & -0.1528 & -0.0550 & -0.0638 & 0.3384 & -0.0719 & 0.5839 & 0.0159 & 0.2564 & 0.1774 & -0.4777 & -0.0623 & -0.0895 & 0.0601 \\
\hline $1 \mathrm{E} 14$ - BCL C302 & 0.3921 & 0.0490 & -0.0332 & -0.2689 & -0.0892 & -0.0020 & 0.2630 & -0.0566 & 0.5775 & 0.0367 & 0.3764 & 0.0944 & -0.3565 & -0.1681 & -0.1525 & 0.0653 \\
\hline 1E6D - BCL C302 & 0.3782 & 0.0533 & 0.0583 & -0.1952 & -0.0740 & -0.0380 & 0.3032 & -0.0520 & 0.4879 & 0.0368 & 0.2350 & 0.2113 & -0.3415 & 0.0491 & -0.1227 & 0.0642 \\
\hline 1EYS - BCL L602 & 0.3696 & 0.0483 & 0.0333 & -0.2302 & -0.1334 & -0.1109 & 0.2063 & -0.0991 & 0.3721 & 0.0770 & 0.0960 & 0.2987 & -0.0824 & -0.0220 & 0.1380 & 0.1172 \\
\hline $1 F 6 N-B C L$ L302 & 0.4508 & 0.0444 & 0.0507 & -0.2407 & -0.0848 & -0.0471 & 0.3588 & -0.0673 & 0.4775 & 0.0714 & 0.1672 & 0.2189 & -0.3465 & -0.0978 & -0.1483 & -0.0224 \\
\hline 1FNP - BCL L302 & 0.4599 & 0.0449 & 0.0555 & -0.2386 & -0.0535 & -0.0806 & 0.3718 & -0.0619 & 0.3882 & 0.0651 & 0.1333 & 0.1403 & -0.2517 & -0.1793 & -0.1264 & -0.0423 \\
\hline 1FNQ - BCL L302 & 0.4275 & 0.0460 & 0.0664 & -0.2221 & -0.0677 & -0.0688 & 0.3395 & -0.0672 & 0.4338 & 0.0653 & 0.1472 & 0.1807 & -0.2785 & -0.2026 & -0.1129 & -0.0496 \\
\hline 1JGW - BCL L851 & 0.4276 & 0.0611 & -0.0748 & -0.1215 & -0.1391 & 0.0202 & 0.3752 & -0.0441 & 0.2104 & 0.0052 & 0.1548 & 0.0789 & -0.1092 & -0.0413 & -0.0210 & -0.0037 \\
\hline 1JGX - BCL L851 & 0.4276 & 0.0611 & -0.0726 & -0.1092 & -0.1744 & 0.0338 & 0.3639 & -0.0409 & 0.1543 & 0.0041 & 0.1060 & 0.0692 & -0.0769 & -0.0344 & -0.0255 & -0.0026 \\
\hline 1JGY - BCL L851 & 0.4186 & 0.0624 & -0.0596 & -0.1116 & -0.2007 & 0.0072 & 0.3424 & -0.0401 & 0.1888 & 0.0051 & 0.1216 & 0.0739 & -0.1136 & -0.0412 & -0.0275 & -0.0076 \\
\hline 1JGZ - BCL L851 & 0.4694 & 0.0637 & 0.1225 & 0.1217 & 0.0227 & -0.1585 & 0.4040 & -0.0413 & 0.2034 & 0.0048 & -0.1639 & -0.0672 & -0.0898 & 0.0195 & -0.0385 & -0.0077 \\
\hline 1JH0 - BCL L851 & 0.3830 & 0.0601 & -0.0804 & -0.1076 & -0.1475 & 0.0013 & 0.3230 & -0.0504 & 0.1301 & 0.0040 & 0.0809 & 0.0437 & -0.0811 & -0.0333 & -0.0275 & -0.0064 \\
\hline 1K6L - BCL L302 & 0.4536 & 0.0425 & 0.0343 & -0.2938 & -0.0789 & -0.1124 & 0.3049 & -0.0801 & 0.6247 & 0.0683 & 0.3929 & 0.2137 & -0.4111 & -0.1081 & -0.0313 & -0.0919 \\
\hline
\end{tabular}




\begin{tabular}{|c|c|c|c|c|c|c|c|c|c|c|c|c|c|c|c|c|}
\hline Cofactor ID & Dip & Sip & B2g & B1g & $\mathrm{Eu}(\mathrm{x})$ & $\mathrm{Eu}(\mathrm{y})$ & A1g & $\mathrm{A} 2 \mathrm{~g}$ & Doop & Soop & $\mathrm{B} 2 \mathrm{u}$ & B1u & $A 2 u$ & $\mathrm{Eg}(\mathrm{x})$ & Eg(y) & A1u \\
\hline $1 \mathrm{~K} 6 \mathrm{~N}-\mathrm{BCL}$ L302 & 0.4541 & 0.0425 & 0.0341 & -0.2939 & -0.0790 & -0.1123 & 0.3056 & -0.0804 & 0.6250 & 0.0684 & 0.3934 & 0.2138 & -0.4112 & -0.1079 & -0.0309 & -0.0921 \\
\hline 1KBY - BCL L851 & 0.4042 & 0.0614 & -0.0891 & -0.1238 & -0.1738 & -0.0001 & 0.3293 & -0.0384 & 0.1935 & 0.0047 & 0.1442 & 0.0797 & -0.0922 & -0.0383 & -0.0182 & -0.0034 \\
\hline 1L9B - BCL L1002 & 0.3123 & 0.0665 & 0.1276 & -0.1925 & -0.0510 & -0.0429 & 0.1747 & -0.0960 & 0.4147 & 0.0166 & 0.3190 & 0.1024 & -0.2242 & -0.0855 & 0.0008 & -0.0464 \\
\hline 1L9J - BCL L1002 & 0.3656 & 0.0672 & 0.0848 & -0.2345 & -0.0516 & -0.0738 & 0.2314 & -0.0991 & 0.2491 & 0.0123 & 0.1748 & 0.0053 & -0.1512 & -0.0747 & -0.0200 & -0.0512 \\
\hline 1L9J - BCL R2002 & 0.4015 & 0.0675 & 0.0755 & -0.2436 & -0.0713 & -0.0636 & 0.2783 & -0.0978 & 0.3087 & 0.0124 & 0.2249 & 0.0436 & -0.1846 & -0.0772 & -0.0285 & -0.0445 \\
\hline 1M3X - BCL L851 & 0.3815 & 0.0616 & -0.0694 & -0.1138 & -0.1395 & -0.0123 & 0.3261 & -0.0428 & 0.2320 & 0.0061 & 0.1756 & 0.0901 & -0.1098 & -0.0452 & -0.0267 & -0.0073 \\
\hline 1MPS - BCL L302 & 0.3868 & 0.0398 & 0.0265 & -0.1674 & -0.0756 & -0.0508 & 0.3280 & -0.0713 & 0.4811 & 0.0713 & 0.1227 & 0.1287 & -0.3999 & -0.1818 & -0.0812 & 0.0156 \\
\hline 10GV - BCL L1282 & 0.4347 & 0.0669 & 0.0607 & -0.2246 & -0.0240 & -0.1555 & 0.3169 & -0.0983 & 0.2680 & 0.0154 & 0.0878 & 0.0767 & -0.2204 & -0.0965 & 0.0016 & -0.0196 \\
\hline 1PCR - BCL L302 & 0.4526 & 0.0424 & 0.0346 & -0.2927 & -0.0782 & -0.1119 & 0.3046 & -0.0809 & 0.6250 & 0.0682 & 0.3930 & 0.2134 & -0.4114 & -0.1091 & -0.0310 & -0.0920 \\
\hline 1PSS - BCL L2 & 0.3045 & 0.0488 & -0.0885 & -0.1093 & -0.0882 & -0.0459 & 0.2420 & -0.0672 & 0.3645 & 0.0164 & 0.2000 & 0.2224 & -0.1886 & -0.0337 & -0.0810 & 0.0108 \\
\hline 1PST - BCL L2 & 0.2791 & 0.0511 & -0.0644 & -0.1023 & -0.0367 & -0.0897 & 0.2261 & -0.0527 & 0.3880 & 0.0173 & 0.3099 & 0.1784 & -0.1355 & -0.0362 & -0.0539 & 0.0080 \\
\hline 1PYH - BCL A302 & 0.3853 & 0.0566 & -0.0220 & -0.3352 & -0.0776 & -0.0818 & 0.1242 & -0.0863 & 0.5192 & 0.0368 & 0.2774 & 0.2176 & -0.2976 & -0.0291 & -0.2327 & 0.0412 \\
\hline 1QOV - BCL L1302 & 0.4287 & 0.0580 & -0.0089 & -0.2861 & -0.0742 & -0.0387 & 0.3056 & -0.0385 & 0.4977 & 0.0379 & 0.2693 & 0.1404 & -0.3481 & 0.0218 & -0.1815 & 0.0293 \\
\hline 1RG5 - BCL L302 & 0.5426 & 0.0516 & 0.0267 & -0.3597 & -0.1361 & -0.0609 & 0.3649 & -0.0943 & 0.4459 & 0.0307 & 0.2442 & 0.1771 & -0.2878 & 0.0014 & -0.1575 & 0.0133 \\
\hline 1RGN - BCL L302 & 0.3755 & 0.0474 & 0.0113 & -0.2674 & -0.0735 & -0.0620 & 0.2111 & -0.1248 & 0.4595 & 0.0286 & 0.1219 & 0.1541 & -0.3515 & -0.1217 & -0.1848 & 0.0077 \\
\hline 1RQK - BCL L302 & 0.3903 & 0.0499 & 0.0116 & -0.3144 & -0.0629 & -0.0613 & 0.1871 & -0.1032 & 0.4177 & 0.0325 & 0.2460 & 0.1447 & -0.2722 & -0.0091 & -0.1365 & 0.0134 \\
\hline 1RVJ - BCL L852 & 0.3678 & 0.0638 & 0.0487 & -0.2034 & -0.0555 & -0.1045 & 0.2600 & -0.0996 & 0.3837 & 0.0250 & 0.0828 & 0.0846 & -0.3229 & -0.1699 & -0.0015 & -0.0095 \\
\hline 1RY5 - BCL L852 & 0.3659 & 0.0663 & 0.0706 & -0.2040 & -0.0242 & -0.1027 & 0.2579 & -0.0982 & 0.4144 & 0.0292 & 0.1486 & 0.1110 & -0.3308 & -0.1670 & -0.0005 & 0.0000 \\
\hline 1RZH - BCL L852 & 0.3470 & 0.0637 & 0.0250 & -0.2004 & -0.0060 & -0.0838 & 0.2479 & -0.1053 & 0.4236 & 0.0285 & 0.1994 & 0.1344 & -0.3005 & -0.1747 & -0.0277 & -0.0072 \\
\hline 1RZZ - BCL L1002 & 0.4029 & 0.0670 & 0.0560 & -0.2024 & -0.0328 & -0.1585 & 0.2874 & -0.0973 & 0.3022 & 0.0177 & 0.1159 & 0.0369 & -0.2506 & -0.1102 & 0.0326 & -0.0226 \\
\hline 1RZZ - BCL R2002 & 0.3952 & 0.0664 & 0.0473 & -0.1945 & -0.0566 & -0.1089 & 0.3020 & -0.0993 & 0.2892 & 0.0187 & 0.0514 & 0.0400 & -0.2525 & -0.1122 & 0.0507 & -0.0222 \\
\hline 1S00 - BCL L1002 & 0.4294 & 0.0628 & 0.0778 & -0.2457 & -0.0589 & -0.1151 & 0.3013 & -0.1023 & 0.3077 & 0.0209 & 0.0470 & 0.0673 & -0.2633 & -0.1310 & 0.0296 & -0.0238 \\
\hline 1S00 - BCL R2002 & 0.4130 & 0.0670 & 0.0698 & -0.2145 & -0.0299 & -0.1205 & 0.3068 & -0.1007 & 0.3375 & 0.0195 & 0.1391 & 0.0460 & -0.2712 & -0.1237 & 0.0566 & -0.0206 \\
\hline 1UMX - BCL L1282 & 0.4720 & 0.0456 & 0.0079 & -0.3432 & -0.1154 & -0.0191 & 0.2876 & -0.0924 & 0.5350 & 0.0344 & 0.2488 & 0.1390 & -0.2941 & -0.0034 & -0.3429 & 0.0305 \\
\hline 1YF6 - BCL L852 & 0.3679 & 0.0472 & 0.1712 & -0.2688 & -0.0504 & -0.0787 & 0.0545 & -0.1487 & 0.5873 & 0.0820 & 0.3700 & 0.1044 & -0.3382 & -0.1907 & -0.1728 & 0.1286 \\
\hline 1YST - BCL L274 & 0.5150 & 0.0862 & 0.0687 & -0.4734 & 0.0044 & -0.1493 & 0.0800 & -0.0879 & 0.2573 & 0.0269 & 0.2162 & -0.0795 & -0.0332 & -0.0889 & 0.0196 & -0.0616 \\
\hline 1Z9J - BCL A851 & 0.3070 & 0.0662 & -0.0549 & -0.1479 & -0.1666 & 0.0028 & 0.1972 & -0.0520 & 0.1562 & 0.0042 & 0.1258 & 0.0639 & -0.0538 & -0.0318 & -0.0204 & -0.0130 \\
\hline $1 Z 9 K-B C L A 851$ & 0.3921 & 0.0609 & -0.1119 & -0.1561 & -0.1531 & 0.1016 & 0.2863 & -0.0336 & 0.1373 & 0.0028 & 0.1154 & 0.0482 & -0.0462 & -0.0300 & -0.0113 & -0.0067 \\
\hline 2BNP - BCL A1282 & 0.2271 & 0.0664 & 0.0492 & -0.0506 & -0.1029 & 0.0070 & 0.0908 & -0.1665 & 0.0378 & 0.0115 & 0.0136 & 0.0063 & -0.0293 & -0.0161 & -0.0006 & 0.0094 \\
\hline 2BNS - BCL A1282 & 0.2856 & 0.0760 & 0.1254 & 0.0296 & -0.1046 & -0.0180 & 0.1861 & -0.1382 & 0.0681 & 0.0182 & 0.0060 & 0.0001 & -0.0654 & -0.0045 & -0.0172 & 0.0022 \\
\hline 2BOZ - BCL L1282 & 0.5802 & 0.0494 & -0.0414 & -0.4030 & -0.1413 & -0.1119 & 0.3579 & -0.1095 & 0.6128 & 0.0381 & 0.3480 & 0.2434 & -0.2838 & -0.0596 & -0.3300 & 0.0465 \\
\hline 2GMR - BCL L302 & 0.4447 & 0.0443 & 0.0623 & -0.1761 & -0.0951 & -0.0853 & 0.3767 & -0.0683 & 0.4996 & 0.0578 & 0.1976 & 0.1618 & -0.3514 & -0.1910 & -0.1443 & 0.0596 \\
\hline 2GNU - BCL L1282 & 0.6186 & 0.0704 & 0.0542 & -0.3151 & -0.1418 & -0.0900 & 0.4869 & -0.1230 & 0.1138 & 0.0196 & 0.0300 & 0.0045 & -0.0919 & -0.0074 & -0.0567 & 0.0178 \\
\hline 2HG3 - BCL L312 & 0.4103 & 0.0463 & 0.0260 & -0.3305 & -0.1269 & -0.0764 & 0.1606 & -0.1035 & 0.5951 & 0.0378 & 0.3501 & 0.1936 & -0.2296 & -0.0781 & -0.3663 & 0.0322 \\
\hline 2HG9 - BCL L312 & 0.4090 & 0.0522 & 0.0555 & -0.3111 & -0.0953 & -0.0928 & 0.1921 & -0.1129 & 0.5719 & 0.0358 & 0.3483 & 0.0908 & -0.2982 & -0.0986 & -0.3134 & 0.0273 \\
\hline $2 \mathrm{HH} 1-\mathrm{BCL}$ L312 & 0.4196 & 0.0519 & -0.0323 & -0.3292 & -0.0449 & -0.1259 & 0.1938 & -0.1058 & 0.5273 & 0.0342 & 0.2839 & 0.1221 & -0.3105 & -0.1472 & -0.2517 & 0.0334 \\
\hline 2HHK - BCL L312 & 0.4078 & 0.0514 & 0.0009 & -0.3008 & -0.0907 & -0.0779 & 0.2226 & -0.1095 & 0.5212 & 0.0381 & 0.2946 & 0.1081 & -0.3342 & -0.1000 & -0.2267 & 0.0068 \\
\hline 2HIT - BCL L312 & 0.4057 & 0.0457 & 0.0384 & -0.3173 & -0.0785 & -0.0631 & 0.2090 & -0.0927 & 0.5515 & 0.0344 & 0.2026 & 0.0972 & -0.3036 & -0.1369 & -0.3772 & 0.0213 \\
\hline 2HJ6 - BCL L312 & 0.3099 & 0.0548 & 0.0983 & -0.2172 & -0.1121 & -0.0536 & 0.1284 & -0.0851 & 0.5819 & 0.0348 & 0.1437 & 0.0655 & -0.4250 & -0.2079 & -0.2995 & 0.0142 \\
\hline $2 J 8 C-B C L$ L1288 & 0.3189 & 0.0493 & -0.0452 & -0.2470 & -0.1027 & -0.0512 & 0.1097 & -0.1160 & 0.5932 & 0.0842 & 0.2641 & 0.1077 & -0.4043 & -0.1731 & -0.2715 & 0.0586 \\
\hline 2J8D - BCL L1282 & 0.3394 & 0.0482 & -0.0651 & -0.2733 & -0.0691 & -0.0766 & 0.0857 & -0.1350 & 0.7244 & 0.0879 & 0.3731 & 0.2989 & -0.3848 & -0.1845 & -0.3288 & 0.0783 \\
\hline 2JIY - BCL L1283 & 0.3828 & 0.0494 & -0.0273 & -0.2894 & -0.1104 & -0.0614 & 0.1986 & -0.0814 & 0.5225 & 0.0351 & 0.2990 & 0.1969 & -0.2846 & -0.0307 & -0.2496 & 0.0242 \\
\hline 2JJO - BCL L1283 & 0.3997 & 0.0477 & 0.0065 & -0.2824 & -0.1244 & -0.0889 & 0.2131 & -0.1054 & 0.4516 & 0.0303 & 0.1887 & 0.2214 & -0.2521 & -0.0478 & -0.2302 & 0.0222 \\
\hline
\end{tabular}




\begin{tabular}{|c|c|c|c|c|c|c|c|c|c|c|c|c|c|c|c|c|}
\hline Cofactor ID & Dip & Sip & B2g & B1g & $\mathrm{Eu}(\mathrm{x})$ & Eu(y) & A1g & A2g & Doop & Soop & $\mathrm{B} 2 \mathrm{u}$ & B1u & $\mathrm{A} 2 \mathrm{u}$ & $\mathrm{Eg}(\mathrm{x})$ & $\mathrm{Eg}(\mathrm{y})$ & A1u \\
\hline 2RCR - BCL L350 & 0.5712 & 0.0533 & 0.3052 & -0.3630 & -0.0339 & -0.0535 & 0.3058 & -0.0620 & 0.2543 & 0.0117 & -0.0699 & 0.2246 & -0.0303 & 0.0654 & -0.0618 & 0.0170 \\
\hline 2UWS - BCL L1286 & 0.3297 & 0.0609 & 0.1197 & -0.2363 & -0.0333 & -0.1303 & -0.0704 & -0.1243 & 0.7939 & 0.1242 & 0.1688 & 0.1538 & -0.5220 & -0.3638 & -0.3458 & 0.2317 \\
\hline 2UWT - BCL L1282 & 0.2880 & 0.0519 & 0.0172 & -0.2389 & -0.0694 & -0.0614 & 0.0442 & -0.1226 & 0.6692 & 0.0918 & 0.2558 & 0.1370 & -0.4400 & -0.2265 & -0.3210 & 0.1252 \\
\hline 2UWU - BCL L1288 & 0.2650 & 0.0507 & -0.0139 & -0.2107 & -0.0657 & -0.0357 & 0.0613 & -0.1278 & 0.6218 & 0.0931 & 0.2580 & 0.2014 & -0.4327 & -0.1263 & -0.2586 & 0.0969 \\
\hline 2UWV - BCL L1286 & 0.3120 & 0.0503 & 0.0341 & -0.2667 & -0.0434 & -0.1008 & 0.0334 & -0.1091 & 0.5929 & 0.0892 & 0.2394 & 0.2032 & -0.3914 & -0.1543 & -0.2581 & 0.0965 \\
\hline 2UWW - BCL L1290 & 0.2718 & 0.0499 & -0.0226 & -0.2264 & -0.0532 & -0.0671 & 0.0518 & -0.1100 & 0.6568 & 0.0937 & 0.2399 & 0.2401 & -0.4570 & -0.1526 & -0.2568 & 0.1344 \\
\hline 2UX3 - BCL L1286 & 0.2430 & 0.0593 & 0.0066 & -0.1882 & -0.0921 & -0.0412 & 0.0202 & -0.1139 & 0.6003 & 0.1043 & 0.1036 & 0.0652 & -0.4600 & -0.2274 & -0.2776 & 0.0714 \\
\hline 2UX4 - BCL L1288 & 0.2177 & 0.0582 & 0.0338 & -0.1194 & -0.0726 & -0.1127 & -0.0174 & -0.1172 & 0.6632 & 0.1146 & 0.1410 & 0.1036 & -0.4504 & -0.2549 & -0.3370 & 0.1670 \\
\hline 2UX5 - BCL L1287 & 0.2739 & 0.0486 & 0.0612 & -0.2350 & -0.0505 & -0.0834 & 0.0142 & -0.0797 & 0.6225 & 0.0844 & 0.2999 & 0.1177 & -0.3742 & -0.1818 & -0.3103 & 0.1201 \\
\hline 2UXJ - BCL L1287 & 0.3006 & 0.0547 & 0.1447 & -0.1990 & -0.0485 & -0.1108 & 0.0102 & -0.1228 & 0.6532 & 0.0976 & 0.2640 & 0.1794 & -0.4325 & -0.1175 & -0.3333 & 0.1134 \\
\hline 2UXK - BCL L1289 & 0.2723 & 0.0585 & -0.0054 & -0.2134 & -0.0211 & -0.1241 & 0.0328 & -0.1080 & 0.6286 & 0.1221 & 0.2530 & 0.0589 & -0.4329 & -0.2196 & -0.2513 & 0.1698 \\
\hline 2UXL - BCL L1285 & 0.3013 & 0.0667 & 0.0252 & -0.2602 & 0.0124 & -0.0779 & 0.0170 & -0.1263 & 0.7357 & 0.1193 & 0.1719 & -0.1359 & -0.5748 & -0.1948 & -0.2856 & 0.2081 \\
\hline 2UXM - BCL L1282 & 0.2787 & 0.0582 & 0.0730 & -0.2080 & -0.1096 & -0.0852 & 0.0203 & -0.0972 & 0.6244 & 0.1133 & 0.0984 & 0.2760 & -0.4460 & -0.2309 & -0.1397 & 0.1798 \\
\hline 2WX5 - BCL L1283 & 0.3434 & 0.0495 & -0.0126 & -0.2040 & -0.1345 & -0.0433 & -0.2138 & -0.1023 & 0.6288 & 0.0651 & 0.3679 & 0.1344 & -0.2390 & -0.1474 & -0.3676 & 0.1673 \\
\hline 3DSY - BCL L502 & 0.4535 & 0.0426 & 0.0343 & -0.2938 & -0.0788 & -0.1123 & 0.3049 & -0.0800 & 0.6248 & 0.0684 & 0.3929 & 0.2142 & -0.4110 & -0.1083 & -0.0312 & -0.0921 \\
\hline 3DTA - BCL L502 & 0.4537 & 0.0425 & 0.0352 & -0.2939 & -0.0785 & -0.1119 & 0.3051 & -0.0804 & 0.6246 & 0.0683 & 0.3931 & 0.2136 & -0.4108 & -0.1078 & -0.0314 & -0.0921 \\
\hline 3DTR - BCL L502 & 0.4534 & 0.0425 & 0.0343 & -0.2939 & -0.0792 & -0.1118 & 0.3049 & -0.0795 & 0.6249 & 0.0682 & 0.3932 & 0.2136 & -0.4111 & -0.1081 & -0.0315 & -0.0924 \\
\hline 3DTS - BCL L502 & 0.4540 & 0.0426 & 0.0338 & -0.2936 & -0.0785 & -0.1119 & 0.3060 & -0.0801 & 0.6247 & 0.0684 & 0.3930 & 0.2142 & -0.4108 & -0.1082 & -0.0308 & -0.0922 \\
\hline 3DU2 - BCL L502 & 0.4535 & 0.0424 & 0.0340 & -0.2936 & -0.0794 & -0.1121 & 0.3050 & -0.0802 & 0.6246 & 0.0683 & 0.3927 & 0.2136 & -0.4114 & -0.1081 & -0.0312 & -0.0918 \\
\hline 3DU3 - BCL L502 & 0.4536 & 0.0425 & 0.0346 & -0.2932 & -0.0783 & -0.1124 & 0.3054 & -0.0809 & 0.6246 & 0.0683 & 0.3930 & 0.2135 & -0.4111 & -0.1084 & -0.0304 & -0.0917 \\
\hline 3DUQ - BCL L502 & 0.4543 & 0.0426 & 0.0343 & -0.2938 & -0.0792 & -0.1125 & 0.3059 & -0.0801 & 0.6252 & 0.0684 & 0.3933 & 0.2142 & -0.4112 & -0.1084 & -0.0311 & -0.0921 \\
\hline 4RCR - BCL L283 & 0.4704 & 0.0321 & 0.0093 & -0.0412 & 0.0165 & -0.0264 & 0.4674 & 0.0045 & 0.4383 & 0.0545 & -0.1066 & 0.2263 & -0.3132 & 0.0843 & -0.1161 & 0.1043 \\
\hline
\end{tabular}

Table 50: Minimum basis normal-mode displacements and conformational parameters of all DM cofactors from crystal structures of Rhodobacter sphaeroides.

\begin{tabular}{|c|c|c|c|c|c|c|c|c|c|c|c|c|c|c|c|c|}
\hline Cofactor ID & Dip & Sip & B2g & B1g & $\mathrm{Eu}(\mathrm{x})$ & $\mathrm{Eu}(\mathrm{y})$ & A1g & $\mathrm{A} 2 \mathrm{~g}$ & Doop & Soop & $\mathrm{B} 2 \mathrm{u}$ & B1u & $\mathrm{A} 2 \mathrm{u}$ & $\mathrm{Eg}(\mathrm{x})$ & $\mathrm{Eg}(\mathrm{y})$ & A1u \\
\hline 1AIG - BCL M310 & 0.2195 & 0.0529 & -0.0848 & -0.0591 & -0.0386 & 0.0306 & 0.1798 & -0.0524 & 0.5709 & 0.0109 & 0.1763 & -0.5362 & -0.0251 & -0.0350 & 0.0213 & -0.0710 \\
\hline 1AIG - BCL 0309 & 0.2874 & 0.0505 & -0.0225 & -0.1271 & -0.0538 & -0.0953 & 0.2242 & -0.0607 & 0.5059 & 0.0081 & 0.1904 & -0.4236 & -0.1558 & -0.1159 & 0.0142 & -0.0486 \\
\hline 1AIJ - BCL M310 & 0.2868 & 0.0538 & -0.0813 & -0.0924 & -0.0306 & -0.0394 & 0.2508 & -0.0417 & 0.4576 & 0.0063 & 0.1534 & -0.3713 & -0.2000 & -0.0753 & 0.0081 & -0.0471 \\
\hline 1AIJ - BCL S310 & 0.2896 & 0.0547 & -0.0323 & -0.0865 & -0.0526 & -0.0630 & 0.2587 & -0.0407 & 0.4303 & 0.0089 & 0.0643 & -0.3731 & -0.1898 & -0.0556 & 0.0083 & -0.0514 \\
\hline 1DS8 - BCL M1003 & 0.3197 & 0.0529 & -0.0089 & -0.1479 & 0.0161 & 0.0236 & 0.2761 & -0.0563 & 0.5475 & 0.0098 & 0.1740 & -0.4810 & -0.1555 & -0.0951 & 0.0247 & -0.0655 \\
\hline 1DS8 - BCL S2003 & 0.3344 & 0.0548 & 0.0284 & -0.1371 & -0.0049 & -0.0967 & 0.2842 & -0.0452 & 0.6457 & 0.0149 & 0.2696 & -0.5346 & -0.1710 & -0.1530 & -0.0588 & -0.0477 \\
\hline 1DV3 - BCL M1003 & 0.3580 & 0.0561 & -0.0498 & -0.1486 & 0.0378 & -0.0606 & 0.3111 & -0.0418 & 0.6492 & 0.0149 & 0.2195 & -0.5329 & -0.2547 & -0.1413 & 0.0159 & -0.0643 \\
\hline 1DV3 - BCL S2003 & 0.3471 & 0.0567 & 0.0028 & -0.1184 & 0.0026 & -0.0478 & 0.3211 & -0.0323 & 0.5507 & 0.0151 & 0.2526 & -0.4100 & -0.2272 & -0.1164 & -0.0679 & -0.0401 \\
\hline 1DV6 - BCL M1003 & 0.3464 & 0.0541 & 0.0411 & -0.1661 & -0.0338 & -0.0412 & 0.2938 & -0.0401 & 0.6063 & 0.0140 & 0.2009 & -0.5076 & -0.2071 & -0.1556 & -0.0103 & -0.0485 \\
\hline 1DV6 - BCL S2003 & 0.3549 & 0.0537 & -0.0604 & -0.0725 & -0.0493 & -0.0639 & 0.3315 & -0.0243 & 0.4480 & 0.0135 & 0.1393 & -0.3606 & -0.2075 & -0.0765 & -0.0064 & -0.0484 \\
\hline 1E14 - BCL C303 & 0.4081 & 0.0483 & 0.0002 & -0.2448 & -0.0655 & -0.0078 & 0.3084 & -0.0845 & 0.5337 & 0.0161 & -0.0863 & -0.4248 & -0.2290 & 0.1013 & -0.1813 & -0.0372 \\
\hline 1E6D - BCL C303 & 0.4427 & 0.0540 & 0.0134 & -0.2579 & -0.0973 & -0.0380 & 0.3355 & -0.0759 & 0.6207 & 0.0130 & 0.3210 & -0.5271 & -0.0293 & -0.0504 & -0.0240 & -0.0200 \\
\hline 1EYS - BCL M603 & 0.4142 & 0.0543 & 0.0437 & -0.2423 & -0.2294 & -0.0932 & 0.1971 & -0.1038 & 0.5242 & 0.0713 & 0.0825 & -0.3768 & -0.0241 & -0.0353 & 0.3514 & -0.0255 \\
\hline $1 F 6 N-B C L$ M801 & 0.4203 & 0.0501 & 0.0777 & -0.2415 & -0.0980 & -0.1045 & 0.2969 & -0.0602 & 0.4833 & 0.0483 & 0.3129 & -0.3119 & -0.0596 & 0.0593 & 0.0575 & -0.1674 \\
\hline 1FNP - BCL M802 & 0.4430 & 0.0482 & 0.0484 & -0.2599 & -0.0971 & -0.1028 & 0.3202 & -0.0619 & 0.5034 & 0.0435 & 0.2854 & -0.3504 & -0.1402 & -0.0412 & 0.0107 & -0.1667 \\
\hline 1FNQ - BCL M802 & 0.3968 & 0.0480 & 0.0759 & -0.2357 & -0.1248 & -0.0860 & 0.2649 & -0.0549 & 0.5459 & 0.0452 & 0.3037 & -0.4068 & -0.0583 & -0.0504 & 0.0149 & -0.1848 \\
\hline
\end{tabular}




\begin{tabular}{|c|c|c|c|c|c|c|c|c|c|c|c|c|c|c|c|c|}
\hline Cofactor ID & Dip & $\delta$ ip & B2g & B1g & $\mathrm{Eu}(\mathrm{x})$ & Eu(y) & A1g & $A 2 g$ & Doop & Soop & B2u & B1u & $\mathrm{A} 2 \mathrm{u}$ & $\mathrm{Eg}(\mathrm{x})$ & $\mathrm{Eg}(\mathrm{y})$ & A1u \\
\hline 1JGW - BCL M852 & 0.4422 & 0.0617 & -0.0155 & -0.1576 & -0.2056 & 0.0857 & 0.3456 & -0.0380 & 0.1489 & 0.0040 & 0.1224 & -0.0750 & -0.0309 & -0.0196 & 0.0061 & -0.0134 \\
\hline 1JGX - BCL M852 & 0.4199 & 0.0649 & -0.0372 & -0.1552 & -0.1621 & 0.0445 & 0.3485 & -0.0336 & 0.0922 & 0.0026 & 0.0648 & -0.0628 & -0.0177 & -0.0048 & 0.0019 & -0.0030 \\
\hline 1JGY - BCL M852 & 0.3718 & 0.0654 & -0.0274 & -0.1417 & -0.1340 & 0.0234 & 0.3120 & -0.0398 & 0.1485 & 0.0029 & 0.1247 & -0.0761 & -0.0182 & -0.0177 & -0.0031 & -0.0069 \\
\hline 1JGZ - BCL M852 & 0.4067 & 0.0627 & -0.0276 & -0.1563 & -0.1808 & 0.0594 & 0.3204 & -0.0370 & 0.1231 & 0.0038 & 0.0976 & -0.0726 & -0.0028 & -0.0157 & 0.0025 & -0.0102 \\
\hline 1JHO - BCL M852 & 0.3866 & 0.0656 & -0.0365 & -0.1566 & -0.1793 & 0.0887 & 0.2870 & -0.0344 & 0.1031 & 0.0029 & 0.0846 & -0.0569 & -0.0101 & -0.0057 & -0.0072 & -0.0062 \\
\hline 1K6L - BCL M502 & 0.3551 & 0.0476 & 0.0157 & -0.2045 & -0.1230 & -0.0944 & 0.2365 & -0.0637 & 0.5968 & 0.0532 & 0.3942 & -0.3404 & -0.1970 & -0.0538 & -0.0215 & -0.2068 \\
\hline $1 \mathrm{~K} 6 \mathrm{~N}$ - BCL M502 & 0.3557 & 0.0475 & 0.0157 & -0.2045 & -0.1240 & -0.0949 & 0.2366 & -0.0642 & 0.5966 & 0.0533 & 0.3938 & -0.3407 & -0.1970 & -0.0541 & -0.0206 & -0.2064 \\
\hline 1L9B - BCL M1003 & 0.3513 & 0.0701 & 0.1327 & -0.2434 & -0.0419 & -0.0422 & 0.1855 & -0.0929 & 0.3479 & 0.0172 & 0.2036 & -0.2002 & -0.1420 & -0.1106 & 0.0374 & -0.0757 \\
\hline 1L9J - BCL M1003 & 0.4260 & 0.0692 & 0.1320 & -0.2810 & -0.0930 & -0.0047 & 0.2593 & -0.0958 & 0.2271 & 0.0115 & 0.1061 & -0.1051 & -0.1403 & -0.0766 & 0.0403 & -0.0459 \\
\hline 1L9J - BCL S2003 & 0.4298 & 0.0692 & 0.1134 & -0.2624 & -0.0935 & -0.0376 & 0.2889 & -0.0971 & 0.2012 & 0.0094 & -0.0271 & -0.1163 & -0.1279 & -0.0734 & 0.0575 & -0.0343 \\
\hline 1M3X - BCL M852 & 0.4304 & 0.0623 & -0.0078 & -0.1526 & -0.1676 & 0.0504 & 0.3607 & -0.0338 & 0.1758 & 0.0039 & 0.1407 & -0.0969 & -0.0355 & -0.0198 & 0.0045 & -0.0079 \\
\hline 1MPS - BCL M802 & 0.4673 & 0.0459 & 0.0394 & -0.2609 & -0.1164 & -0.0605 & 0.3553 & -0.0726 & 0.5981 & 0.0475 & 0.2760 & -0.5092 & -0.0972 & -0.0231 & -0.0612 & -0.0926 \\
\hline 10GV - BCL M1303 & 0.3817 & 0.0713 & 0.0959 & -0.2072 & -0.0581 & -0.0685 & 0.2786 & -0.0889 & 0.3016 & 0.0145 & 0.1632 & -0.1735 & -0.1388 & -0.0844 & 0.0730 & -0.0502 \\
\hline 1PCR - BCL M801 & 0.3554 & 0.0476 & 0.0163 & -0.2040 & -0.1227 & -0.0941 & 0.2376 & -0.0633 & 0.5950 & 0.0531 & 0.3929 & -0.3392 & -0.1963 & -0.0534 & -0.0196 & -0.2068 \\
\hline 1PSS - BCL M3 & 0.2186 & 0.0518 & -0.0652 & -0.0537 & -0.0539 & -0.0404 & 0.1856 & -0.0411 & 0.4652 & 0.0100 & 0.0920 & -0.4111 & -0.1896 & -0.0212 & -0.0260 & -0.0432 \\
\hline 1PYH - BCL B303 & 0.3838 & 0.0538 & 0.0139 & -0.3229 & -0.0643 & -0.0409 & 0.1682 & -0.0937 & 0.5453 & 0.0172 & 0.3057 & -0.4235 & -0.0837 & -0.1039 & -0.0382 & -0.0728 \\
\hline 1QOV - BCL L1303 & 0.4501 & 0.0552 & 0.0592 & -0.3198 & -0.0362 & -0.0439 & 0.3007 & -0.0560 & 0.5190 & 0.0184 & 0.3066 & -0.3792 & -0.0795 & -0.1177 & -0.0699 & -0.0804 \\
\hline 1RG5 - BCL M502 & 0.4867 & 0.0561 & -0.0130 & -0.3086 & -0.0827 & -0.0350 & 0.3560 & -0.0818 & 0.5276 & 0.0129 & 0.3037 & -0.4141 & -0.0611 & -0.0979 & 0.0102 & -0.0343 \\
\hline $1 R G N-B C L$ M909 & 0.3324 & 0.0602 & -0.0322 & -0.1967 & -0.0923 & -0.0096 & 0.2306 & -0.0949 & 0.5593 & 0.0155 & 0.2779 & -0.4318 & -0.0328 & -0.1967 & -0.0891 & -0.0373 \\
\hline 1RQK - BCL M502 & 0.3218 & 0.0551 & 0.0321 & -0.2795 & -0.0621 & 0.0202 & 0.1118 & -0.0877 & 0.5896 & 0.0171 & 0.2808 & -0.4721 & -0.0544 & -0.2034 & -0.0159 & -0.0355 \\
\hline 1RVJ - BCL M853 & 0.3732 & 0.0703 & 0.0405 & -0.1799 & -0.0566 & -0.0146 & 0.3104 & -0.0744 & 0.3154 & 0.0129 & 0.1042 & -0.2402 & -0.1425 & -0.0830 & 0.0347 & -0.0501 \\
\hline 1RY5 - BCL M853 & 0.4050 & 0.0686 & 0.0954 & -0.2551 & -0.0301 & -0.0275 & 0.2835 & -0.0888 & 0.4563 & 0.0182 & 0.2521 & -0.3102 & -0.1667 & -0.1073 & 0.0627 & -0.0725 \\
\hline 1RZH - BCL M853 & 0.4498 & 0.0553 & 0.0589 & -0.3195 & -0.0362 & -0.0433 & 0.3007 & -0.0560 & 0.5195 & 0.0183 & 0.3063 & -0.3802 & -0.0798 & -0.1179 & -0.0696 & -0.0802 \\
\hline 1RZZ - BCL M1003 & 0.3865 & 0.0688 & 0.1296 & -0.2375 & -0.0520 & -0.0259 & 0.2545 & -0.0898 & 0.3136 & 0.0156 & 0.1278 & -0.2121 & -0.1433 & -0.0809 & 0.0887 & -0.0459 \\
\hline 1RZZ - BCL S2003 & 0.4062 & 0.0726 & 0.1106 & -0.2385 & -0.0292 & -0.0945 & 0.2804 & -0.0867 & 0.2699 & 0.0139 & 0.0592 & -0.1905 & -0.1358 & -0.0809 & 0.0776 & -0.0454 \\
\hline $1 S 00$ - BCL M1003 & 0.4009 & 0.0705 & 0.1319 & -0.2250 & -0.0422 & -0.0751 & 0.2773 & -0.0916 & 0.2977 & 0.0155 & 0.0544 & -0.2185 & -0.1414 & -0.0791 & 0.0965 & -0.0487 \\
\hline $1 S 00$ - BCL S2003 & 0.3864 & 0.0700 & 0.1079 & -0.2248 & -0.0291 & -0.1028 & 0.2599 & -0.0902 & 0.2491 & 0.0135 & 0.0054 & -0.1661 & -0.1471 & -0.0707 & 0.0777 & -0.0419 \\
\hline 1UMX - BCL M1304 & 0.4539 & 0.0582 & 0.1215 & -0.3426 & -0.0987 & -0.0980 & 0.2010 & -0.1189 & 0.4517 & 0.0168 & 0.3171 & -0.3053 & 0.0551 & -0.0740 & -0.0027 & -0.0418 \\
\hline 1YF6 - BCL M853 & 0.3439 & 0.0585 & -0.0479 & -0.2872 & -0.0964 & 0.0002 & 0.0994 & -0.1197 & 0.7078 & 0.0646 & 0.2993 & -0.5617 & -0.1744 & -0.1773 & -0.0070 & -0.1847 \\
\hline 1YST - BCL M308 & 0.5612 & 0.0721 & 0.1643 & -0.5218 & -0.0954 & 0.0339 & -0.0459 & -0.0579 & 0.2931 & 0.0143 & 0.2239 & -0.1653 & -0.0684 & 0.0428 & 0.0235 & -0.0376 \\
\hline 1Z9J - BCL B852 & 0.3693 & 0.0697 & -0.0972 & -0.2344 & -0.1382 & 0.0558 & 0.2195 & -0.0401 & 0.0833 & 0.0028 & -0.0714 & -0.0417 & -0.0074 & 0.0013 & 0.0070 & -0.0016 \\
\hline 1Z9K - BCL B852 & 0.3228 & 0.0622 & -0.0640 & -0.1764 & -0.1638 & 0.0407 & 0.1974 & -0.0390 & 0.0225 & 0.0028 & 0.0018 & -0.0086 & 0.0060 & -0.0191 & 0.0043 & 0.0032 \\
\hline 2BNP - BCL B1303 & 0.4239 & 0.0896 & 0.3688 & 0.1399 & -0.0685 & 0.0900 & 0.0203 & -0.1044 & 0.0604 & 0.0076 & 0.0071 & -0.0363 & -0.0062 & -0.0386 & 0.0218 & -0.0165 \\
\hline 2BNS - BCL B1303 & 0.4077 & 0.0845 & 0.2825 & 0.1281 & -0.1186 & 0.1138 & 0.1798 & -0.1034 & 0.0569 & 0.0119 & 0.0062 & -0.0338 & -0.0333 & -0.0268 & 0.0069 & -0.0134 \\
\hline 2BOZ - BCL M1304 & 0.5506 & 0.0573 & -0.0576 & -0.3871 & -0.1053 & -0.0131 & 0.3574 & -0.1046 & 0.6155 & 0.0194 & 0.3873 & -0.4427 & -0.0810 & -0.1473 & -0.0481 & -0.0477 \\
\hline 2GMR - BCL M309 & 0.4207 & 0.0465 & 0.0665 & -0.2113 & -0.1130 & -0.0540 & 0.3281 & -0.0672 & 0.4781 & 0.0300 & 0.1483 & -0.3588 & -0.1676 & -0.1544 & 0.0204 & -0.1598 \\
\hline 2GNU - BCL M1303 & 0.5770 & 0.0754 & 0.1890 & -0.2752 & -0.0964 & 0.0756 & 0.4478 & -0.0769 & 0.1342 & 0.0161 & 0.0190 & -0.0920 & -0.0540 & -0.0614 & 0.0280 & -0.0416 \\
\hline $2 \mathrm{HG} 3$ - BCL M313 & 0.3861 & 0.0617 & 0.0872 & -0.2472 & -0.0098 & 0.0927 & 0.2484 & -0.1000 & 0.5754 & 0.0187 & 0.2738 & -0.4926 & -0.0122 & -0.0601 & -0.0263 & -0.0949 \\
\hline 2HG9 - BCL M313 & 0.3138 & 0.0620 & -0.0983 & -0.1982 & 0.0004 & 0.0810 & 0.1868 & -0.0895 & 0.7413 & 0.0238 & 0.3897 & -0.6149 & -0.0479 & -0.0944 & -0.0773 & -0.0492 \\
\hline $2 \mathrm{HH} 1$ - BCL M313 & 0.2779 & 0.0629 & -0.0582 & -0.1151 & -0.0338 & -0.0084 & 0.2328 & -0.0720 & 0.7147 & 0.0224 & 0.3884 & -0.5782 & -0.1007 & -0.0976 & -0.0518 & -0.0576 \\
\hline $2 \mathrm{HHK}-\mathrm{BCL}$ M313 & 0.3865 & 0.0556 & -0.1288 & -0.2424 & -0.0554 & -0.0037 & 0.2656 & -0.0190 & 0.6524 & 0.0215 & 0.4019 & -0.4962 & -0.0245 & -0.1006 & -0.0748 & -0.0397 \\
\hline 2HIT - BCL M313 & 0.4032 & 0.0568 & 0.1075 & -0.0632 & -0.0439 & -0.0679 & 0.3595 & -0.1061 & 0.6521 & 0.0231 & 0.3892 & -0.5082 & 0.0462 & -0.1015 & -0.0355 & -0.0428 \\
\hline $2 \mathrm{HJ} 6-\mathrm{BCL}$ M313 & 0.2734 & 0.0587 & -0.0167 & -0.1330 & -0.1341 & -0.0291 & 0.1628 & -0.1071 & 0.7505 & 0.0247 & 0.3908 & -0.6047 & -0.0058 & -0.1988 & 0.0263 & -0.0684 \\
\hline
\end{tabular}




\begin{tabular}{|c|c|c|c|c|c|c|c|c|c|c|c|c|c|c|c|c|}
\hline Cofactor ID & Dip & Sip & B2g & B1g & $\mathrm{Eu}(\mathrm{x})$ & Eu(y) & A1g & $A 2 g$ & Doop & Soop & $\mathrm{B} 2 \mathrm{u}$ & B1u & $\mathrm{A} 2 \mathrm{u}$ & $\mathrm{Eg}(\mathrm{x})$ & $\mathrm{Eg}(\mathrm{y})$ & A1u \\
\hline $2 \mathrm{~J} 8 \mathrm{C}-\mathrm{BCL}$ M1304 & 0.3378 & 0.0539 & 0.0021 & -0.2277 & -0.1203 & -0.0444 & 0.1879 & -0.1026 & 0.6806 & 0.0392 & 0.4009 & -0.4873 & -0.1965 & -0.0925 & 0.0427 & -0.1269 \\
\hline 2J8D - BCL M1309 & 0.3616 & 0.0497 & 0.0863 & -0.2800 & -0.0866 & -0.0177 & 0.1545 & -0.1149 & 0.6529 & 0.0548 & 0.3685 & -0.4490 & -0.1806 & -0.1801 & 0.0557 & -0.1441 \\
\hline $2 \mathrm{JIY}-\mathrm{BCL}$ M1303 & 0.3647 & 0.0545 & 0.0134 & -0.2264 & -0.0465 & -0.0021 & 0.2691 & -0.0840 & 0.4891 & 0.0195 & 0.2359 & -0.3865 & -0.0387 & -0.1203 & 0.1200 & -0.0620 \\
\hline $2 \mathrm{JIY}-\mathrm{BCL}$ M1303 & 0.4142 & 0.0567 & 0.0211 & -0.3401 & -0.0144 & 0.0351 & 0.2127 & -0.0936 & 0.8693 & 0.0238 & 0.3532 & -0.6541 & -0.1044 & -0.3614 & -0.2439 & -0.0453 \\
\hline 2JJO - BCL M1304 & 0.3250 & 0.0586 & -0.0009 & -0.2501 & -0.0078 & -0.0143 & 0.1818 & -0.0985 & 0.5755 & 0.0187 & 0.2866 & -0.4581 & -0.0109 & -0.1921 & -0.0346 & -0.0322 \\
\hline 2RCR - BCL M400 & 0.4293 & 0.0564 & 0.0548 & -0.2590 & -0.0970 & -0.0098 & 0.3132 & -0.0816 & 0.3314 & 0.0160 & -0.2153 & -0.1327 & -0.0749 & 0.0592 & 0.1899 & 0.0254 \\
\hline 2UWS - BCL M1304 & 0.3208 & 0.0596 & -0.0217 & -0.2609 & -0.0866 & 0.0238 & -0.0990 & -0.1284 & 0.5590 & 0.1203 & 0.3233 & -0.3066 & -0.1494 & 0.0221 & 0.0932 & -0.2871 \\
\hline 2UWT - BCL M1304 & 0.2852 & 0.0565 & 0.0674 & -0.2230 & -0.0756 & -0.0406 & 0.0143 & -0.1396 & 0.7054 & 0.0614 & 0.3371 & -0.5653 & -0.1378 & -0.1071 & -0.0188 & -0.1833 \\
\hline 2UWU - BCL M1304 & 0.3065 & 0.0533 & 0.0654 & -0.2579 & -0.0488 & 0.0004 & 0.1002 & -0.1036 & 0.7158 & 0.0456 & 0.3914 & -0.5487 & -0.1606 & -0.1540 & -0.0131 & -0.0917 \\
\hline 2UWV - BCL M1304 & 0.3080 & 0.0560 & 0.0187 & -0.2790 & -0.0526 & 0.0271 & 0.0359 & -0.1090 & 0.7130 & 0.0463 & 0.4304 & -0.5182 & -0.1119 & -0.1480 & 0.0006 & -0.1421 \\
\hline 2UWW - BCL M1305 & 0.3049 & 0.0513 & 0.0651 & -0.2503 & -0.0541 & -0.0153 & 0.0954 & -0.1174 & 0.7234 & 0.0468 & 0.3648 & -0.5470 & -0.2192 & -0.1242 & -0.0843 & -0.1432 \\
\hline 2UX3 - BCL M1304 & 0.2917 & 0.0597 & 0.0223 & -0.2397 & -0.0776 & -0.0287 & 0.0594 & -0.1295 & 0.8342 & 0.0502 & 0.3188 & -0.7137 & -0.2142 & -0.1535 & 0.0085 & -0.1241 \\
\hline 2UX4 - BCL M1304 & 0.2558 & 0.0582 & -0.0274 & -0.1638 & -0.0673 & -0.0283 & -0.0292 & -0.1780 & 0.8600 & 0.0808 & 0.2779 & -0.7625 & -0.1979 & -0.1211 & 0.1028 & -0.1285 \\
\hline 2UX5 - BCL M1305 & 0.3436 & 0.0579 & 0.0744 & -0.3069 & -0.0478 & -0.0052 & 0.0909 & -0.0883 & 0.7179 & 0.0503 & 0.3887 & -0.5573 & -0.1309 & -0.1239 & 0.0863 & -0.1174 \\
\hline 2UXJ - BCL M1304 & 0.3275 & 0.0510 & 0.0174 & -0.2903 & -0.0490 & -0.0050 & 0.0658 & -0.1264 & 0.6800 & 0.0684 & 0.3786 & -0.4812 & -0.1909 & -0.1137 & 0.0192 & -0.1943 \\
\hline 2UXK - BCL M1304 & 0.3010 & 0.0546 & 0.0359 & -0.2546 & -0.0295 & -0.0079 & 0.0564 & -0.1427 & 0.7310 & 0.0903 & 0.3517 & -0.5209 & -0.2764 & -0.1071 & -0.0801 & -0.2124 \\
\hline 2UXL - BCL M1304 & 0.3066 & 0.0614 & 0.0355 & -0.2621 & -0.0242 & -0.0718 & -0.0267 & -0.1327 & 0.7250 & 0.1098 & 0.2030 & -0.5218 & -0.3889 & -0.2436 & 0.0385 & 0.0086 \\
\hline 2UXM - BCL M1304 & 0.3137 & 0.0640 & -0.0901 & -0.2406 & -0.1213 & -0.0043 & -0.0065 & -0.1328 & 0.9139 & 0.0917 & 0.3276 & -0.7688 & -0.2065 & -0.1131 & 0.1673 & -0.2312 \\
\hline 2WX5 - BCL M1303 & 0.2648 & 0.0441 & -0.0148 & -0.1401 & -0.0519 & -0.1301 & -0.1417 & -0.1030 & 0.5996 & 0.0450 & 0.2492 & -0.4629 & -0.1245 & -0.0129 & 0.0198 & -0.2589 \\
\hline 3DSY - BCL M502 & 0.3552 & 0.0476 & 0.0155 & -0.2045 & -0.1226 & -0.0944 & 0.2368 & -0.0638 & 0.5969 & 0.0532 & 0.3945 & -0.3405 & -0.1971 & -0.0537 & -0.0210 & -0.2064 \\
\hline 3DTA - BCL M502 & 0.3556 & 0.0476 & 0.0156 & -0.2046 & -0.1234 & -0.0946 & 0.2368 & -0.0642 & 0.5975 & 0.0533 & 0.3946 & -0.3410 & -0.1972 & -0.0535 & -0.0215 & -0.2069 \\
\hline 3DTR - BCL M502 & 0.3550 & 0.0475 & 0.0158 & -0.2042 & -0.1228 & -0.0940 & 0.2368 & -0.0636 & 0.5969 & 0.0533 & 0.3946 & -0.3403 & -0.1972 & -0.0530 & -0.0210 & -0.2066 \\
\hline 3DTS - BCL M502 & 0.3557 & 0.0476 & 0.0157 & -0.2043 & -0.1238 & -0.0951 & 0.2367 & -0.0644 & 0.5968 & 0.0532 & 0.3940 & -0.3413 & -0.1960 & -0.0539 & -0.0203 & -0.2066 \\
\hline 3DU2 - BCL M502 & 0.3549 & 0.0475 & 0.0148 & -0.2041 & -0.1224 & -0.0948 & 0.2368 & -0.0635 & 0.5970 & 0.0532 & 0.3942 & -0.3403 & -0.1975 & -0.0543 & -0.0210 & -0.2069 \\
\hline 3DU3 - BCL M502 & 0.3559 & 0.0476 & 0.0157 & -0.2040 & -0.1244 & -0.0951 & 0.2370 & -0.0641 & 0.5969 & 0.0532 & 0.3943 & -0.3410 & -0.1967 & -0.0536 & -0.0211 & -0.2061 \\
\hline 3DUQ - BCL M502 & 0.3556 & 0.0477 & 0.0157 & -0.2048 & -0.1236 & -0.0951 & 0.2365 & -0.0631 & 0.5965 & 0.0533 & 0.3940 & -0.3403 & -0.1971 & -0.0548 & -0.0205 & -0.2063 \\
\hline 4RCR - BCL M310 & 0.4911 & 0.0361 & -0.0187 & -0.0729 & 0.0347 & -0.0235 & 0.4833 & -0.0118 & 0.6497 & 0.0499 & 0.3028 & -0.4903 & -0.2372 & -0.1071 & 0.1246 & -0.0824 \\
\hline
\end{tabular}

\subsection{Blastochloris Viridis bacteriochlorin cofactor NSDs}

8.2.1 Minimum Basis

Table 51: Minimum basis normal-mode displacements and conformational parameters of all BA cofactors from crystal structures of Blastochloris viridis.

\begin{tabular}{|c|c|c|c|c|c|c|c|c|c|c|c|c|c|c|c|c|}
\hline Cofactor ID & Dip & Sip & B2g & B1g & $\mathrm{Eu}(\mathrm{x})$ & $\mathrm{Eu}(\mathrm{y})$ & A1g & A2g & Doop & 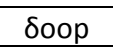 & $\mathrm{B} 2 \mathrm{u}$ & B1u & $\mathrm{A} 2 \mathrm{u}$ & $\mathrm{Eg}(\mathrm{x})$ & $\mathrm{Eg}(\mathrm{y})$ & A1u \\
\hline 1DXR - BCB L401 & 0.4577 & 0.0405 & 0.0365 & -0.3396 & -0.1465 & -0.0943 & 0.2405 & -0.0680 & 0.4287 & 0.0380 & 0.0403 & 0.0633 & 0.0065 & -0.4170 & 0.0168 & 0.0634 \\
\hline 1PRC - BCB L604 & 0.2998 & 0.0472 & 0.0674 & -0.1271 & -0.0911 & -0.0692 & 0.2218 & -0.0830 & 0.3019 & 0.0394 & 0.1285 & 0.1168 & -0.0856 & -0.2169 & 0.0698 & 0.0412 \\
\hline 1R2C - BCB L304 & 0.4282 & 0.0425 & 0.0700 & -0.2552 & -0.1371 & -0.0632 & 0.2925 & -0.0705 & 0.4264 & 0.0443 & 0.0515 & 0.1304 & -0.1755 & -0.3065 & 0.1698 & 0.0927 \\
\hline $1 V R N$ - BCB L404 & 0.4577 & 0.0430 & 0.0569 & -0.2671 & -0.1183 & -0.0633 & 0.3337 & -0.0744 & 0.4822 & 0.0439 & 0.1453 & 0.1305 & -0.0666 & -0.4179 & 0.0954 & 0.0788 \\
\hline $215 N-B C B$ L401 & 0.4610 & 0.0511 & 0.1045 & -0.2378 & -0.1177 & -0.0694 & 0.3407 & -0.1015 & 0.4916 & 0.0542 & 0.0374 & 0.1917 & 0.0053 & -0.4484 & -0.0128 & 0.0472 \\
\hline 2JBL - BCB L1275 & 0.4483 & 0.0434 & 0.0658 & -0.2574 & -0.1038 & -0.0598 & 0.3336 & -0.0687 & 0.5172 & 0.0421 & 0.0969 & 0.1673 & -0.1616 & -0.4348 & 0.0962 & 0.0754 \\
\hline 2PRC - BCB L304 & 0.4361 & 0.0398 & 0.0317 & -0.2727 & -0.1688 & -0.0320 & 0.2803 & -0.0816 & 0.4685 & 0.0445 & 0.0795 & 0.1079 & -0.0944 & -0.4156 & 0.1242 & 0.0670 \\
\hline 2WJM - BCB L1275 & 0.3556 & 0.0555 & 0.1857 & -0.0921 & -0.0021 & -0.1073 & 0.2624 & -0.0558 & 0.3394 & 0.0526 & 0.1035 & 0.1158 & -0.0536 & -0.2730 & 0.0410 & 0.1094 \\
\hline
\end{tabular}




\begin{tabular}{|c|c|c|c|c|c|c|c|c|c|c|c|c|c|c|c|c|}
\hline Cofactor ID & Dip & Sip & B2g & B1g & $\mathrm{Eu}(\mathrm{x})$ & Eu(y) & A1g & $A 2 g$ & Doop & Soop & $\mathrm{B} 2 \mathrm{u}$ & B1u & $\mathrm{A} 2 \mathrm{u}$ & $\mathrm{Eg}(\mathrm{x})$ & $\mathrm{Eg}(\mathrm{y})$ & A1u \\
\hline 2WJN - BCB L1275 & 0.3934 & 0.0567 & 0.1247 & -0.2501 & 0.1131 & -0.1165 & 0.2159 & -0.0605 & 0.4083 & 0.0650 & 0.1385 & 0.1465 & -0.0301 & -0.3368 & 0.0862 & 0.0656 \\
\hline 3D38 - BCB L401 & 0.4476 & 0.0485 & 0.1597 & -0.2732 & -0.1179 & -0.1144 & 0.2494 & -0.1050 & 0.3813 & 0.0358 & -0.0657 & 0.1787 & -0.1202 & -0.3012 & -0.0432 & 0.0457 \\
\hline 3G7F - BCB L401 & 0.4295 & 0.0481 & 0.1701 & -0.2407 & -0.1289 & -0.0383 & 0.2609 & -0.1072 & 0.3820 & 0.0513 & 0.1441 & 0.0823 & -0.0562 & -0.3388 & 0.0116 & 0.0178 \\
\hline 3PRC - BCB L304 & 0.4128 & 0.0402 & 0.0391 & -0.2884 & -0.1395 & -0.0634 & 0.2370 & -0.0779 & 0.4827 & 0.0557 & 0.2220 & 0.0686 & 0.0236 & -0.4073 & 0.0985 & 0.0536 \\
\hline 5PRC - BCB L304 & 0.3615 & 0.0418 & -0.0100 & -0.2666 & -0.1498 & -0.0415 & 0.1726 & -0.0744 & 0.4154 & 0.0512 & 0.1569 & 0.0936 & -0.0579 & -0.3447 & 0.1002 & 0.0836 \\
\hline 6PRC - BCB L304 & 0.4188 & 0.0393 & 0.0261 & -0.3139 & -0.1499 & -0.0855 & 0.1990 & -0.0824 & 0.4530 & 0.0510 & 0.1489 & 0.0927 & 0.0146 & -0.4045 & 0.0382 & 0.0957 \\
\hline 7PRC - BCB L304 & 0.4344 & 0.0402 & 0.0629 & -0.3462 & -0.1668 & -0.0352 & 0.1770 & -0.0668 & 0.5752 & 0.0610 & 0.3437 & 0.1532 & 0.0467 & -0.4035 & 0.1250 & 0.0930 \\
\hline
\end{tabular}

Table 52: Minimum basis normal-mode displacements and conformational parameters of all BB cofactors from crystal structures of Blastochloris viridis.

\begin{tabular}{|c|c|c|c|c|c|c|c|c|c|c|c|c|c|c|c|c|}
\hline Cofactor ID & Dip & Sip & B2g & B1g & $\mathrm{Eu}(\mathrm{x})$ & Eu(y) & A1g & A2g & Doop & Soop & B2u & B1u & $\mathrm{A} 2 \mathrm{u}$ & $\mathrm{Eg}(\mathrm{x})$ & $\mathrm{Eg}(\mathrm{y})$ & A1u \\
\hline 1DXR - BCB M400 & 0.4516 & 0.0419 & 0.0220 & -0.2919 & -0.0906 & 0.0084 & 0.3220 & -0.0790 & 0.4471 & 0.0451 & 0.0847 & 0.0114 & -0.0372 & -0.4345 & 0.0464 & 0.0143 \\
\hline 1PRC - BCB M601 & 0.3533 & 0.0492 & 0.0859 & -0.1604 & -0.1037 & -0.0454 & 0.2691 & -0.0804 & 0.1875 & 0.0458 & 0.0619 & 0.0503 & 0.0065 & -0.1562 & 0.0600 & -0.0272 \\
\hline 1R2C - BCB M1301 & 0.4663 & 0.0397 & 0.0566 & -0.2977 & -0.1141 & -0.0242 & 0.3266 & -0.0726 & 0.3731 & 0.0614 & 0.1120 & 0.0027 & -0.1717 & -0.2659 & 0.1622 & 0.0119 \\
\hline $1 \mathrm{VRN}$ - BCB M401 & 0.4273 & 0.0417 & 0.0730 & -0.2373 & -0.1001 & -0.0349 & 0.3232 & -0.0724 & 0.3815 & 0.0497 & -0.0084 & 0.0157 & -0.1532 & -0.3453 & 0.0465 & 0.0194 \\
\hline $215 N-B C B$ M400 & 0.4203 & 0.0491 & 0.0427 & -0.2724 & -0.0769 & -0.0616 & 0.2849 & -0.0986 & 0.4511 & 0.0463 & -0.1011 & 0.1467 & -0.1801 & -0.3721 & 0.0268 & -0.0141 \\
\hline 2JBL - BCB L1278 & 0.4259 & 0.042 & 0.0643 & -0.2291 & -0.1067 & -0.0099 & 0.3281 & -0.0750 & 0.5413 & 0.0496 & -0.0419 & 0.0166 & -0.3232 & -0.4023 & 0.1514 & 0.0422 \\
\hline 2PRC - BCB M805 & 0.4284 & 0.04 & 0.0902 & -0.3069 & -0.0657 & -0.0161 & 0.2654 & -0.0786 & 0.4866 & 0.0497 & 0.0484 & 0.0640 & -0.1548 & -0.4455 & 0.0849 & 0.0275 \\
\hline 2WJM - BCB M1324 & 0.2845 & 0.0569 & 0.0467 & -0.1321 & 0.0519 & -0.0173 & 0.2343 & -0.0586 & 0.3269 & 0.0478 & 0.1233 & 0.1253 & -0.1395 & -0.2250 & 0.0640 & 0.0425 \\
\hline 2WJN - BCB M1324 & 0.2334 & 0.057 & 0.0432 & -0.1278 & -0.0405 & -0.0631 & 0.1702 & -0.0410 & 0.3525 & 0.0613 & 0.1550 & 0.0028 & -0.1650 & -0.2639 & 0.0461 & 0.0348 \\
\hline 3D38 - BCB M400 & 0.4047 & 0.0511 & -0.0379 & -0.2981 & -0.0773 & -0.0055 & 0.2310 & -0.1188 & 0.4574 & 0.0417 & -0.0971 & -0.1217 & -0.1815 & -0.3899 & -0.0041 & -0.0016 \\
\hline $3 G 7 F-B C B M 400$ & 0.4373 & 0.0534 & 0.1277 & -0.3233 & -0.1110 & -0.0015 & 0.2182 & -0.1025 & 0.4771 & 0.0536 & 0.0181 & 0.2400 & -0.1861 & -0.3627 & 0.0557 & -0.0191 \\
\hline 3PRC - BCB M805 & 0.449 & 0.0408 & 0.0901 & -0.2733 & -0.1142 & -0.0311 & 0.3139 & -0.0790 & 0.4253 & 0.0503 & 0.0371 & 0.0165 & -0.1808 & -0.3712 & 0.0914 & 0.0205 \\
\hline 5PRC - BCB M805 & 0.3589 & 0.04 & 0.0587 & -0.2606 & -0.0646 & -0.0211 & 0.2156 & -0.0797 & 0.3919 & 0.0624 & -0.0508 & -0.0008 & -0.1009 & -0.3679 & 0.0685 & -0.0287 \\
\hline $6 \mathrm{PRC}-\mathrm{BCB} M 805$ & 0.3815 & 0.0403 & 0.0349 & -0.2313 & -0.0871 & -0.0225 & 0.2767 & -0.0783 & 0.3973 & 0.0629 & 0.0561 & 0.0083 & -0.1559 & -0.3413 & 0.1162 & 0.0196 \\
\hline 7PRC - BCB M805 & 0.3691 & 0.0397 & 0.0585 & -0.2480 & -0.0961 & -0.0638 & 0.2290 & -0.0742 & 0.4673 & 0.0575 & -0.0206 & -0.0246 & -0.1600 & -0.4373 & -0.0176 & -0.0115 \\
\hline
\end{tabular}

Table 53: Minimum basis normal-mode displacements and conformational parameters of all DL cofactors from crystal structures of Blastochloris viridis.

\begin{tabular}{|c|c|c|c|c|c|c|c|c|c|c|c|c|c|c|c|c|}
\hline Cofactor ID & Dip & Sip & B2g & B1g & $\mathrm{Eu}(\mathrm{x})$ & $\mathrm{Eu}(\mathrm{y})$ & A1g & $A 2 g$ & Doop & 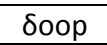 & $\mathrm{B} 2 \mathrm{u}$ & B1u & $\mathrm{A} 2 \mathrm{u}$ & $\mathrm{Eg}(\mathrm{x})$ & $\mathrm{Eg}(\mathrm{y})$ & A1u \\
\hline 1DXR - BCB L400 & 0.3642 & 0.0432 & 0.0761 & -0.1851 & -0.1309 & -0.0213 & 0.2594 & -0.0877 & 0.6675 & 0.0369 & -0.1356 & -0.6097 & -0.0545 & 0.0164 & 0.0788 & -0.2142 \\
\hline 1PRC - BCB L602 & 0.3796 & 0.0515 & 0.0714 & -0.2989 & -0.1082 & -0.0525 & 0.1565 & -0.1035 & 0.4142 & 0.0401 & -0.1540 & -0.3335 & -0.0628 & -0.0963 & 0.0452 & -0.1460 \\
\hline 1R2C - BCB L302 & 0.3952 & 0.0431 & 0.0736 & -0.2640 & -0.1524 & -0.0099 & 0.2274 & -0.0775 & 0.6191 & 0.0339 & -0.2842 & -0.5396 & 0.0273 & 0.0025 & 0.0048 & -0.1026 \\
\hline 1VRN - BCB L402 & 0.4232 & 0.0401 & 0.0549 & -0.2417 & -0.1364 & -0.0311 & 0.3055 & -0.0687 & 0.7993 & 0.0395 & -0.2273 & -0.7262 & 0.0554 & 0.0256 & 0.0251 & -0.2354 \\
\hline $215 \mathrm{~N}-\mathrm{BCB} L 400$ & 0.3712 & 0.0471 & 0.0433 & -0.1956 & -0.0853 & -0.0246 & 0.2801 & -0.1067 & 0.8137 & 0.0527 & -0.2829 & -0.6912 & -0.0597 & -0.1813 & 0.1546 & -0.2099 \\
\hline 2JBL - BCB L1274 & 0.4053 & 0.0402 & 0.0522 & -0.2438 & -0.1313 & -0.0361 & 0.2789 & -0.0762 & 0.8316 & 0.0302 & -0.2768 & -0.7508 & -0.0485 & 0.0314 & -0.0439 & -0.2142 \\
\hline 2PRC - BCB L302 & 0.3387 & 0.0461 & 0.0588 & -0.1843 & -0.1140 & 0.0011 & 0.2357 & -0.0933 & 0.7697 & 0.0434 & -0.3183 & -0.6704 & -0.0628 & 0.0918 & 0.0242 & -0.1691 \\
\hline 2WJM - BCB L1274 & 0.3344 & 0.0600 & 0.0958 & -0.1111 & -0.0502 & -0.0534 & 0.2801 & -0.0807 & 0.7138 & 0.0372 & -0.1294 & -0.6433 & 0.0907 & 0.0178 & 0.1678 & -0.2052 \\
\hline 2WJN - BCB L1274 & 0.3556 & 0.0629 & 0.0536 & -0.2123 & -0.0755 & -0.0178 & 0.2610 & -0.0665 & 0.7064 & 0.0420 & -0.1611 & -0.6453 & 0.0398 & 0.0298 & 0.1125 & -0.2038 \\
\hline 3D38 - BCB L400 & 0.4314 & 0.0507 & 0.1269 & -0.2275 & -0.1473 & -0.0827 & 0.2810 & -0.1036 & 0.8735 & 0.0500 & -0.3069 & -0.7682 & -0.1401 & -0.0955 & 0.1295 & -0.1823 \\
\hline $3 G 7 F-B C B L 400$ & 0.4065 & 0.0427 & 0.0733 & -0.2609 & -0.1680 & -0.0439 & 0.2198 & -0.1156 & 0.8106 & 0.0360 & -0.2221 & -0.7575 & -0.0100 & -0.0477 & 0.0891 & -0.1536 \\
\hline
\end{tabular}




\begin{tabular}{|c|c|c|c|c|c|c|c|c|c|c|c|c|c|c|c|c|}
\hline Cofactor ID & Dip & Sip & B2g & B1g & $\mathrm{Eu}(\mathrm{x})$ & Eu(y) & A1g & $A 2 g$ & Doop & Soop & $\mathrm{B} 2 \mathrm{u}$ & B1u & $\mathrm{A} 2 \mathrm{u}$ & $\mathrm{Eg}(\mathrm{x})$ & $\mathrm{Eg}(\mathrm{y})$ & A1u \\
\hline 3PRC - BCB L302 & 0.4032 & 0.0433 & 0.0230 & -0.2658 & -0.1418 & -0.0245 & 0.2530 & -0.0819 & 0.7212 & 0.0357 & -0.2206 & -0.6532 & -0.0345 & 0.0889 & 0.0164 & -0.1880 \\
\hline 5PRC - BCB L302 & 0.2949 & 0.0406 & 0.0075 & -0.1809 & -0.1143 & -0.0034 & 0.1811 & -0.0913 & 0.7247 & 0.0332 & -0.2736 & -0.6334 & -0.1208 & -0.0160 & 0.0946 & -0.1591 \\
\hline 6PRC - BCB L302 & 0.3283 & 0.0416 & -0.0162 & -0.1929 & -0.1255 & -0.0718 & 0.2046 & -0.0866 & 0.6822 & 0.0312 & -0.2425 & -0.6105 & -0.0836 & 0.0633 & 0.0201 & -0.1501 \\
\hline 7PRC - BCB L302 & 0.3831 & 0.0408 & 0.0724 & -0.2407 & -0.1544 & -0.0396 & 0.2231 & -0.0920 & 0.8808 & 0.0338 & -0.2702 & -0.8092 & -0.1099 & 0.0712 & 0.0437 & -0.1698 \\
\hline
\end{tabular}

Table 54: Minimum basis normal-mode displacements and conformational parameters of all DM cofactors from crystal structures of Blastochloris viridis.

\begin{tabular}{|c|c|c|c|c|c|c|c|c|c|c|c|c|c|c|c|c|}
\hline Cofactor ID & Dip & Sip & B2g & B1g & $\mathrm{Eu}(\mathrm{x})$ & $\mathrm{Eu}(\mathrm{y})$ & A1g & $\mathrm{A} 2 \mathrm{~g}$ & Doop & Soop & $\mathrm{B} 2 \mathrm{u}$ & B1u & $\mathrm{A} 2 \mathrm{u}$ & $\mathrm{Eg}(\mathrm{x})$ & $\mathrm{Eg}(\mathrm{y})$ & A1u \\
\hline 1DXR - BCB M401 & 0.3393 & 0.0405 & 0.0407 & -0.1697 & -0.1475 & -0.0320 & 0.2391 & -0.0688 & 0.8485 & 0.0343 & -0.1055 & -0.7896 & 0.0154 & -0.1413 & 0.1457 & -0.2097 \\
\hline 1PRC - BCB M603 & 0.2809 & 0.0521 & 0.0714 & -0.0871 & -0.0834 & -0.2157 & 0.0381 & -0.1063 & 0.6630 & 0.0518 & 0.1016 & -0.6127 & -0.0368 & 0.0465 & 0.0876 & -0.2064 \\
\hline 1R2C - BCB M1303 & 0.3299 & 0.0362 & 0.0960 & -0.1696 & -0.2055 & -0.0276 & 0.1493 & -0.0744 & 1.0678 & 0.0392 & -0.2000 & -1.0022 & 0.0344 & -0.1134 & 0.1458 & -0.2462 \\
\hline 1VRN - BCB M403 & 0.3625 & 0.0391 & 0.0489 & -0.2320 & -0.1281 & -0.0248 & 0.2300 & -0.0722 & 1.1001 & 0.0363 & -0.0507 & -1.0594 & 0.0506 & -0.0177 & 0.0999 & -0.2692 \\
\hline $215 N$ - BCB M401 & 0.3278 & 0.0500 & -0.0401 & -0.2469 & -0.0651 & -0.0429 & 0.1691 & -0.1011 & 1.1229 & 0.0576 & -0.0776 & -1.0376 & -0.1059 & -0.1277 & 0.2509 & -0.2962 \\
\hline 2JBL - BCB M1324 & 0.3689 & 0.0395 & 0.0389 & -0.1859 & -0.2027 & -0.0187 & 0.2312 & -0.0717 & 1.0042 & 0.0269 & -0.1067 & -0.9563 & -0.0135 & -0.0724 & 0.1875 & -0.2046 \\
\hline 2PRC - BCB M806 & 0.2626 & 0.0373 & -0.0289 & -0.1333 & -0.1417 & -0.0981 & 0.1183 & -0.0817 & 1.0783 & 0.0311 & -0.1390 & -1.0334 & -0.1197 & -0.0009 & 0.1803 & -0.1695 \\
\hline 2WJM - BCB M1325 & 0.2634 & 0.0610 & 0.1694 & -0.0586 & 0.0055 & 0.0077 & 0.1861 & -0.0501 & 1.0496 & 0.0559 & 0.1314 & -0.9883 & 0.0021 & -0.0162 & 0.1559 & -0.2883 \\
\hline 2WJN - BCB M1325 & 0.2371 & 0.0575 & 0.1009 & -0.1309 & -0.0261 & -0.0302 & 0.1592 & -0.0443 & 1.0096 & 0.0532 & 0.0926 & -0.9426 & -0.0525 & -0.0102 & 0.1628 & -0.3046 \\
\hline 3D38 - BCB M401 & 0.3846 & 0.0430 & 0.1239 & -0.2622 & -0.0469 & -0.0577 & 0.2131 & -0.1134 & 0.9181 & 0.0541 & -0.2597 & -0.7987 & -0.0166 & -0.1838 & 0.2161 & -0.2382 \\
\hline $3 P R C-B C B$ M806 & 0.3314 & 0.0379 & 0.0460 & -0.1440 & -0.1327 & -0.0312 & 0.2481 & -0.0826 & 0.9802 & 0.0372 & -0.1396 & -0.9353 & -0.0174 & -0.0157 & 0.0849 & -0.2426 \\
\hline 5PRC - BCB M806 & 0.3374 & 0.0400 & 0.0239 & -0.1881 & -0.1807 & -0.0262 & 0.1983 & -0.0720 & 0.9599 & 0.0325 & -0.0442 & -0.9183 & -0.0737 & -0.0609 & 0.1690 & -0.1966 \\
\hline $6 P R C-B C B$ M806 & 0.3119 & 0.0404 & -0.0144 & -0.1992 & -0.1294 & -0.0179 & 0.1848 & -0.0785 & 1.0536 & 0.0371 & -0.0860 & -1.0110 & -0.1147 & -0.0691 & 0.0508 & -0.2449 \\
\hline 7PRC - BCB M806 & 0.3803 & 0.0454 & 0.0220 & -0.2106 & -0.1436 & -0.0517 & 0.2647 & -0.0801 & 0.9954 & 0.0436 & -0.0113 & -0.9643 & -0.1297 & -0.0297 & 0.0976 & -0.1835 \\
\hline
\end{tabular}

8.2.2 Extended Basis (Lowest Energy)

Table 55: Extended conformational parameters and lowest energy basis normal-mode displacements of all BA cofactors from crystal structures of Blastochloris viridis.

\begin{tabular}{|c|c|c|c|c|c|c|c|c|c|c|c|c|c|c|c|c|}
\hline Cofactor ID & Dip_e & Sip_e & B2g_e & B1g_e & $\mathrm{Eu}(\mathrm{x}) \_\mathrm{e}$ & Eu(y)_e & A1g_e & A2g_e & Doop_e & Soop_e & B2u_e & B1u_e & A2u_e & $E g(x) \_e$ & Eg(y)_e & Alu_e \\
\hline 1DXR - BCB L401 & 0.4845 & 0.0240 & 0.0373 & -0.3408 & -0.1471 & -0.0933 & 0.2413 & -0.0672 & 0.4501 & 0.0312 & 0.0417 & 0.0633 & 0.0032 & -0.4159 & 0.0193 & 0.0634 \\
\hline 1PRC - BCB L604 & 0.3503 & 0.0360 & 0.0673 & -0.1266 & -0.0910 & -0.0681 & 0.2228 & -0.0811 & 0.3201 & 0.0401 & 0.1280 & 0.1168 & -0.0857 & -0.2160 & 0.0700 & 0.0412 \\
\hline 1R2C - BCB L304 & 0.4606 & 0.0253 & 0.0700 & -0.2562 & -0.1379 & -0.0618 & 0.2939 & -0.0704 & 0.4918 & 0.0255 & 0.0511 & 0.1304 & -0.1809 & -0.2993 & 0.1729 & 0.0928 \\
\hline 1VRN - BCB L404 & 0.4886 & 0.0252 & 0.0574 & -0.2683 & -0.1192 & -0.0619 & 0.3347 & -0.0740 & 0.5250 & 0.0274 & 0.1480 & 0.1305 & -0.0702 & -0.4136 & 0.0972 & 0.0788 \\
\hline $215 N-B C B ~ L 401$ & 0.5162 & 0.0247 & 0.1052 & -0.2371 & -0.1179 & -0.0670 & 0.3428 & -0.1013 & 0.5026 & 0.0526 & 0.0388 & 0.1917 & -0.0011 & -0.4473 & -0.0112 & 0.0472 \\
\hline 2JBL - BCB L1275 & 0.4800 & 0.0263 & 0.0660 & -0.2582 & -0.1049 & -0.0585 & 0.3351 & -0.0681 & 0.5474 & 0.0284 & 0.0984 & 0.1673 & -0.1691 & -0.4317 & 0.0949 & 0.0754 \\
\hline 2PRC - BCB L304 & 0.4625 & 0.0247 & 0.0323 & -0.2741 & -0.1694 & -0.0310 & 0.2810 & -0.0810 & 0.5037 & 0.0320 & 0.0811 & 0.1079 & -0.1001 & -0.4129 & 0.1266 & 0.0670 \\
\hline 2WJM - BCB L1275 & 0.3827 & 0.0487 & 0.1859 & -0.0899 & -0.0030 & -0.1039 & 0.2605 & -0.0561 & 0.3714 & 0.0492 & 0.1046 & 0.1158 & -0.0557 & -0.2730 & 0.0419 & 0.1094 \\
\hline 2WJN - BCB L1275 & 0.4247 & 0.0490 & 0.1246 & -0.2473 & 0.1136 & -0.1130 & 0.2140 & -0.0599 & 0.4450 & 0.0575 & 0.1406 & 0.1465 & -0.0280 & -0.3370 & 0.0877 & 0.0656 \\
\hline 3D38 - BCB L401 & 0.4982 & 0.0256 & 0.1610 & -0.2726 & -0.1176 & -0.1124 & 0.2509 & -0.1046 & 0.3883 & 0.0362 & -0.0648 & 0.1787 & -0.1236 & -0.3011 & -0.0432 & 0.0457 \\
\hline 3G7F - BCB L401 & 0.4802 & 0.0263 & 0.1711 & -0.2399 & -0.1294 & -0.0368 & 0.2625 & -0.1065 & 0.3924 & 0.0511 & 0.1450 & 0.0823 & -0.0610 & -0.3380 & 0.0111 & 0.0178 \\
\hline 3PRC - BCB L304 & 0.4421 & 0.0241 & 0.0395 & -0.2896 & -0.1400 & -0.0622 & 0.2379 & -0.0773 & 0.5445 & 0.0340 & 0.2252 & 0.0686 & 0.0169 & -0.4026 & 0.1006 & 0.0536 \\
\hline 5PRC - BCB L304 & 0.3963 & 0.0250 & -0.0095 & -0.2680 & -0.1505 & -0.0401 & 0.1733 & -0.0738 & 0.4749 & 0.0326 & 0.1575 & 0.0936 & -0.0673 & -0.3411 & 0.1028 & 0.0836 \\
\hline 6PRC - BCB L304 & 0.4477 & 0.0221 & 0.0264 & -0.3151 & -0.1508 & -0.0842 & 0.2000 & -0.0818 & 0.5003 & 0.0362 & 0.1511 & 0.0927 & 0.0057 & -0.4041 & 0.0394 & 0.0957 \\
\hline
\end{tabular}




\begin{tabular}{|c|c|c|c|c|c|c|c|c|c|c|c|c|c|c|c|c|}
\hline Cofa & Dip_e & Sip_e & B2g_e & B1g_e & $E u(x) \_e$ & Eu(y)_e & A1g_e & A2g_e & Doop_e & Soop_e & B2u_e & B1u_e & A2u_e & $\mathrm{Eg}(\mathrm{x}) \_\mathrm{e}$ & Eg(y)_e & 1u_e \\
\hline 7PRC - BCB L304 & 0.4618 & 0.0245 & 0.0634 & -0.3478 & -0.1673 & -0.0341 & 0.1782 & -0.0659 & 0.6558 & 0.0313 & 0.3488 & 0.1532 & 0.0377 & -0.4018 & 0.1204 & 930 \\
\hline
\end{tabular}

Table 56: Extended conformational parameters and lowest energy basis normal-mode displacements of all BB cofactors from crystal structures of Blastochloris viridis.

\begin{tabular}{|c|c|c|c|c|c|c|c|c|c|c|c|c|c|c|c|c|}
\hline Cofactor ID & Dip_e & Sip_e & B2g_e & B1g_e & $\mathrm{Eu}(\mathrm{x}) \_\mathrm{e}$ & $\mathrm{Eu}(\mathrm{y}) \_\mathrm{e}$ & A1g_e & A2g_e & Doop_e & Soop_e & B2u_e & B1u_e & A2u_e & $E g(x) \_e$ & $\mathrm{Eg}(\mathrm{y}) \_\mathrm{e}$ & A1u_e \\
\hline 1DXR - BCB M400 & 0.4817 & 0.0250 & 0.0225 & -0.2933 & -0.0917 & 0.0099 & 0.3225 & -0.0786 & 0.4935 & 0.0324 & 0.0867 & 0.0114 & -0.0384 & -0.4291 & 0.0495 & 0.0143 \\
\hline 1PRC - BCB M601 & 0.4072 & 0.0332 & 0.0853 & -0.1601 & -0.1040 & -0.0442 & 0.2681 & -0.0789 & 0.2294 & 0.0443 & 0.0627 & 0.0503 & 0.0078 & -0.1544 & 0.0599 & -0.0272 \\
\hline 1R2C - BCB M1301 & 0.4886 & 0.0270 & 0.0572 & -0.2990 & -0.1145 & -0.0234 & 0.3281 & -0.0721 & 0.4917 & 0.0308 & 0.1136 & 0.0027 & -0.1806 & -0.2569 & 0.1593 & 0.0119 \\
\hline 1VRN - BCB M401 & 0.4585 & 0.0247 & 0.0733 & -0.2384 & -0.1009 & -0.0335 & 0.3242 & -0.0720 & 0.4597 & 0.0276 & -0.0070 & 0.0157 & -0.1531 & -0.3371 & 0.0500 & 0.0194 \\
\hline $215 N-B C B M 400$ & 0.4727 & 0.0262 & 0.0439 & -0.2722 & -0.0770 & -0.0605 & 0.2865 & -0.0981 & 0.4631 & 0.0440 & -0.0996 & 0.1466 & -0.1835 & -0.3708 & 0.0274 & -0.0141 \\
\hline 2JBL - BCB L1278 & 0.4551 & 0.0277 & 0.0645 & -0.2302 & -0.1074 & -0.0083 & 0.3300 & -0.0747 & 0.6043 & 0.0269 & -0.0415 & 0.0166 & -0.3276 & -0.3928 & 0.1532 & 0.0422 \\
\hline 2PRC - BCB M805 & 0.4559 & 0.0249 & 0.0906 & -0.3080 & -0.0668 & -0.0148 & 0.2660 & -0.0783 & 0.5455 & 0.0296 & 0.0495 & 0.0640 & -0.1584 & -0.4372 & 0.0847 & 0.0275 \\
\hline 2WJM - BCB M1324 & 0.3237 & 0.0516 & 0.0494 & -0.1296 & 0.0515 & -0.0138 & 0.2327 & -0.0593 & 0.3490 & 0.0469 & 0.1236 & 0.1253 & -0.1411 & -0.2241 & 0.0652 & 0.0425 \\
\hline 2WJN - BCB M1324 & 0.2781 & 0.0489 & 0.0450 & -0.1267 & -0.0423 & -0.0602 & 0.1690 & -0.0401 & 0.3881 & 0.0586 & 0.1562 & 0.0028 & -0.1669 & -0.2641 & 0.0475 & 0.0348 \\
\hline 3D38 - BCB M400 & 0.4685 & 0.0260 & -0.0358 & -0.2975 & -0.0777 & -0.0050 & 0.2334 & -0.1184 & 0.4620 & 0.0411 & -0.0959 & -0.1217 & -0.1843 & -0.3900 & -0.0039 & -0.0016 \\
\hline $3 G 7 F-B C B M 400$ & 0.5039 & 0.0240 & 0.1283 & -0.3224 & -0.1111 & 0.0004 & 0.2196 & -0.1027 & 0.4952 & 0.0513 & 0.0190 & 0.2400 & -0.1900 & -0.3612 & 0.0562 & -0.0191 \\
\hline 3PRC - BCB M805 & 0.4753 & 0.0259 & 0.0908 & -0.2742 & -0.1148 & -0.0302 & 0.3148 & -0.0784 & 0.4897 & 0.0312 & 0.0376 & 0.0165 & -0.1861 & -0.3644 & 0.0869 & 0.0205 \\
\hline 5PRC - BCB M805 & 0.3939 & 0.0233 & 0.0593 & -0.2617 & -0.0653 & -0.0199 & 0.2163 & -0.0794 & 0.5268 & 0.0312 & -0.0505 & -0.0008 & -0.0998 & -0.3549 & 0.0688 & -0.0287 \\
\hline $6 \mathrm{PRC}-\mathrm{BCB} M 805$ & 0.4136 & 0.0248 & 0.0356 & -0.2326 & -0.0873 & -0.0213 & 0.2776 & -0.0781 & 0.5252 & 0.0311 & 0.0562 & 0.0083 & -0.1594 & -0.3290 & 0.1155 & 0.0196 \\
\hline 7PRC - BCB M805 & 0.3966 & 0.0265 & 0.0599 & -0.2490 & -0.0967 & -0.0629 & 0.2300 & -0.0733 & 0.5626 & 0.0314 & -0.0198 & -0.0246 & -0.1606 & -0.4262 & -0.0215 & -0.0115 \\
\hline
\end{tabular}

Table 57: Extended conformational parameters and lowest energy basis normal-mode displacements of all DL cofactors from crystal structures of Blastochloris viridis.

\begin{tabular}{|c|c|c|c|c|c|c|c|c|c|c|c|c|c|c|c|c|}
\hline Cofactor ID & Dip_e & Sip_e & B2g_e & B1g_e & $\mathrm{Eu}(\mathrm{x}) \_\mathrm{e}$ & $\mathrm{Eu}(\mathrm{y}) \_\mathrm{e}$ & A1g_e & A2g_e & Doop_e & Soop_e & B2u_e & B1u_e & A2u_e & $\mathrm{Eg}(\mathrm{x}) \_\mathrm{e}$ & Eg(y)_e & A1u_e \\
\hline 1DXR - BCB L400 & 0.4080 & 0.0231 & 0.0770 & -0.1862 & -0.1315 & -0.0201 & 0.2601 & -0.0865 & 0.6993 & 0.0151 & -0.1345 & -0.6097 & -0.0560 & 0.0137 & 0.0775 & -0.2142 \\
\hline 1PRC - BCB L602 & 0.4272 & 0.0345 & 0.0711 & -0.2967 & -0.1094 & -0.0499 & 0.1576 & -0.1017 & 0.4737 & 0.0176 & -0.1544 & -0.3335 & -0.0636 & -0.0956 & 0.0454 & -0.1460 \\
\hline $1 R 2 C-B C B$ L302 & 0.4301 & 0.0264 & 0.0737 & -0.2648 & -0.1531 & -0.0087 & 0.2282 & -0.0761 & 0.6480 & 0.0136 & -0.2833 & -0.5396 & 0.0326 & 0.0022 & 0.0103 & -0.1026 \\
\hline $1 V R N$ - BCB L402 & 0.4539 & 0.0225 & 0.0554 & -0.2427 & -0.1372 & -0.0299 & 0.3066 & -0.0676 & 0.8283 & 0.0215 & -0.2264 & -0.7262 & 0.0546 & 0.0229 & 0.0240 & -0.2354 \\
\hline $215 N-B C B ~ L 400$ & 0.4275 & 0.0247 & 0.0448 & -0.1950 & -0.0856 & -0.0232 & 0.2822 & -0.1055 & 0.8586 & 0.0359 & -0.2808 & -0.6912 & -0.0591 & -0.1841 & 0.1538 & -0.2099 \\
\hline 2JBL - BCB L1274 & 0.4365 & 0.0231 & 0.0530 & -0.2445 & -0.1321 & -0.0348 & 0.2801 & -0.0753 & 0.8462 & 0.0198 & -0.2772 & -0.7508 & -0.0493 & 0.0327 & -0.0457 & -0.2142 \\
\hline 2PRC - BCB L302 & 0.3868 & 0.0271 & 0.0597 & -0.1849 & -0.1150 & 0.0021 & 0.2361 & -0.0926 & 0.8059 & 0.0184 & -0.3198 & -0.6704 & -0.0683 & 0.0906 & 0.0178 & -0.1691 \\
\hline 2WJM - BCB L1274 & 0.3804 & 0.0501 & 0.0981 & -0.1097 & -0.0506 & -0.0492 & 0.2778 & -0.0807 & 0.7402 & 0.0229 & -0.1289 & -0.6433 & 0.0909 & 0.0161 & 0.1651 & -0.2052 \\
\hline 2WJN - BCB L1274 & 0.4247 & 0.0459 & 0.0556 & -0.2107 & -0.0759 & -0.0144 & 0.2585 & -0.0636 & 0.7458 & 0.0214 & -0.1596 & -0.6453 & 0.0422 & 0.0266 & 0.1108 & -0.2038 \\
\hline 3D38 - BCB L400 & 0.4952 & 0.0225 & 0.1284 & -0.2273 & -0.1474 & -0.0808 & 0.2836 & -0.1022 & 0.9069 & 0.0384 & -0.3050 & -0.7682 & -0.1367 & -0.0991 & 0.1286 & -0.1823 \\
\hline $3 G 7 F-B C B$ L400 & 0.4434 & 0.0251 & 0.0738 & -0.2606 & -0.1678 & -0.0429 & 0.2211 & -0.1146 & 0.8309 & 0.0271 & -0.2207 & -0.7575 & -0.0088 & -0.0507 & 0.0891 & -0.1536 \\
\hline 3PRC - BCB L302 & 0.4402 & 0.0242 & 0.0237 & -0.2666 & -0.1427 & -0.0234 & 0.2539 & -0.0806 & 0.7510 & 0.0148 & -0.2201 & -0.6532 & -0.0370 & 0.0843 & 0.0149 & -0.1880 \\
\hline 5PRC - BCB L302 & 0.3365 & 0.0247 & 0.0084 & -0.1819 & -0.1148 & -0.0023 & 0.1817 & -0.0901 & 0.7450 & 0.0198 & -0.2742 & -0.6334 & -0.1203 & -0.0148 & 0.0955 & -0.1591 \\
\hline 6PRC - BCB L302 & 0.3705 & 0.0223 & -0.0154 & -0.1938 & -0.1264 & -0.0705 & 0.2048 & -0.0854 & 0.7041 & 0.0201 & -0.2428 & -0.6105 & -0.0844 & 0.0628 & 0.0198 & -0.1501 \\
\hline 7PRC - BCB L302 & 0.4188 & 0.0238 & 0.0733 & -0.2412 & -0.1549 & -0.0387 & 0.2236 & -0.0916 & 0.8994 & 0.0189 & -0.2717 & -0.8092 & -0.1115 & 0.0721 & 0.0409 & -0.1698 \\
\hline
\end{tabular}


Table 58: Extended conformational parameters and lowest energy basis normal-mode displacements of all DM cofactors from crystal structures of Blastochloris viridis.

\begin{tabular}{|c|c|c|c|c|c|c|c|c|c|c|c|c|c|c|c|c|}
\hline Cofactor ID & Dip_e & Sip_e & B2g_e & B1g_e & $\mathrm{Eu}(\mathrm{x}) \_\mathrm{e}$ & Eu(y)_e & A1g_e & A2g_e & Doop_e & Soop_e & B2u_e & B1u_e & A2u_e & $\mathrm{Eg}(\mathrm{x}) \_\mathrm{e}$ & Eg(y)_e & A1u_e \\
\hline 1DXR - BCB M401 & 0.3817 & 0.0211 & 0.0405 & -0.1704 & -0.1481 & -0.0307 & 0.2406 & -0.0681 & 0.8672 & 0.0163 & -0.1052 & -0.7896 & 0.0168 & -0.1426 & 0.1460 & -0.2097 \\
\hline 1PRC - BCB M603 & 0.3634 & 0.0314 & 0.0703 & -0.0844 & -0.0847 & -0.2148 & 0.0379 & -0.1052 & 0.7232 & 0.0145 & 0.1016 & -0.6127 & -0.0341 & 0.0480 & 0.0855 & -0.2064 \\
\hline 1R2C - BCB M1303 & 0.3621 & 0.0219 & 0.0957 & -0.1702 & -0.2056 & -0.0266 & 0.1506 & -0.0741 & 1.0864 & 0.0205 & -0.2011 & -1.0022 & 0.0369 & -0.1082 & 0.1436 & -0.2462 \\
\hline $1 \mathrm{VRN}$ - BCB M403 & 0.4044 & 0.0189 & 0.0491 & -0.2328 & -0.1288 & -0.0234 & 0.2316 & -0.0716 & 1.1192 & 0.0109 & -0.0507 & -1.0594 & 0.0498 & -0.0193 & 0.1007 & -0.2692 \\
\hline $215 N-B C B$ M401 & 0.4032 & 0.0214 & -0.0388 & -0.2464 & -0.0657 & -0.0412 & 0.1716 & -0.1001 & 1.1564 & 0.0368 & -0.0765 & -1.0376 & -0.1040 & -0.1299 & 0.2493 & -0.2962 \\
\hline 2JBL - BCB M1324 & 0.4052 & 0.0223 & 0.0390 & -0.1869 & -0.2029 & -0.0174 & 0.2329 & -0.0712 & 1.0151 & 0.0108 & -0.1071 & -0.9563 & -0.0127 & -0.0724 & 0.1888 & -0.2046 \\
\hline 2PRC - BCB M806 & 0.3070 & 0.0228 & -0.0282 & -0.1338 & -0.1426 & -0.0969 & 0.1196 & -0.0809 & 1.0896 & 0.0180 & -0.1401 & -1.0334 & -0.1206 & -0.0035 & 0.1793 & -0.1695 \\
\hline 2WJM - BCB M1325 & 0.3402 & 0.0453 & 0.1699 & -0.0577 & 0.0048 & 0.0111 & 0.1853 & -0.0500 & 1.0914 & 0.0257 & 0.1322 & -0.9883 & 0.0000 & -0.0200 & 0.1567 & -0.2883 \\
\hline 2WJN - BCB M1325 & 0.3198 & 0.0423 & 0.1030 & -0.1304 & -0.0270 & -0.0276 & 0.1589 & -0.0414 & 1.0480 & 0.0269 & 0.0937 & -0.9426 & -0.0539 & -0.0148 & 0.1633 & -0.3046 \\
\hline 3D38 - BCB M401 & 0.4304 & 0.0223 & 0.1246 & -0.2619 & -0.0467 & -0.0559 & 0.2148 & -0.1110 & 0.9604 & 0.0354 & -0.2578 & -0.7987 & -0.0157 & -0.1867 & 0.2147 & -0.2382 \\
\hline $3 P R C-B C B M 806$ & 0.3695 & 0.0216 & 0.0461 & -0.1449 & -0.1334 & -0.0299 & 0.2491 & -0.0818 & 0.9981 & 0.0157 & -0.1393 & -0.9353 & -0.0165 & -0.0176 & 0.0831 & -0.2426 \\
\hline 5PRC - BCB M806 & 0.3808 & 0.0220 & 0.0243 & -0.1892 & -0.1810 & -0.0247 & 0.1996 & -0.0711 & 0.9731 & 0.0159 & -0.0441 & -0.9183 & -0.0727 & -0.0612 & 0.1674 & -0.1966 \\
\hline $6 \mathrm{PRC}-\mathrm{BCB} M 806$ & 0.3606 & 0.0207 & -0.0133 & -0.2003 & -0.1301 & -0.0166 & 0.1865 & -0.0778 & 1.0714 & 0.0169 & -0.0863 & -1.0110 & -0.1173 & -0.0695 & 0.0509 & -0.2449 \\
\hline 7PRC - BCB M806 & 0.4232 & 0.0270 & 0.0239 & -0.2118 & -0.1440 & -0.0506 & 0.2667 & -0.0791 & 1.0237 & 0.0166 & -0.0116 & -0.9643 & -0.1369 & -0.0249 & 0.0908 & -0.1835 \\
\hline
\end{tabular}

\subsubsection{Extended Basis (Next-to-lowest Energy)}

Table 59: Extended conformational parameters and next-to-lowest energy basis normal-mode displacements of all BA cofactors from crystal structures of Blastochloris viridis.

\begin{tabular}{|c|c|c|c|c|c|c|c|c|c|c|c|c|}
\hline Cofactor ID & B2g_2 & B1g_2 & $\mathrm{Eu}(\mathrm{x}) \_2$ & $\mathrm{Eu}(\mathrm{y}) \_2$ & A1g_2 & A2g_2 & B2u_2 & B1u_2 & A2u_2 & $E g(x) \_2$ & $\mathrm{Eg}(\mathrm{y}) \_2$ & A1u_2 \\
\hline 1DXR - BCB L401 & -0.0113 & -0.0905 & -0.0579 & 0.1156 & 0.0009 & -0.0163 & 0.0648 & -0.0546 & -0.0550 & 0.0568 & 0.0697 & -0.0200 \\
\hline 1PRC - BCB L604 & 0.0271 & -0.0411 & -0.0701 & 0.1532 & 0.0294 & 0.0363 & -0.0093 & -0.0607 & -0.0011 & 0.0673 & 0.0264 & -0.0431 \\
\hline 1R2C - BCB L304 & 0.0308 & -0.1028 & -0.0569 & 0.1160 & -0.0008 & -0.0240 & -0.0254 & -0.0624 & -0.0985 & 0.1956 & 0.0802 & -0.0354 \\
\hline 1VRN - BCB L404 & 0.0109 & -0.1059 & -0.0553 & 0.1203 & -0.0069 & -0.0189 & 0.1296 & -0.0432 & -0.0671 & 0.1282 & 0.0522 & -0.0307 \\
\hline $215 N-B C B ~ L 401$ & 0.0821 & -0.0862 & -0.0944 & 0.1668 & -0.0513 & 0.0169 & 0.0100 & -0.0191 & -0.0221 & 0.0929 & -0.0140 & 0.0187 \\
\hline 2JBL - BCB L1275 & 0.0165 & -0.1017 & -0.0543 & 0.1251 & -0.0003 & -0.0165 & 0.0688 & -0.0310 & -0.1319 & 0.0944 & -0.0144 & -0.0192 \\
\hline 2PRC - BCB L304 & 0.0007 & -0.1000 & -0.0400 & 0.1076 & -0.0031 & -0.0262 & 0.0659 & -0.0814 & -0.1003 & 0.0833 & 0.0779 & -0.0175 \\
\hline 2WJM - BCB L1275 & -0.0302 & 0.0667 & 0.0110 & 0.0911 & 0.0631 & 0.0498 & 0.0365 & -0.0840 & -0.0150 & 0.0885 & 0.0616 & -0.0344 \\
\hline 2WJN - BCB L1275 & -0.0233 & 0.0737 & -0.0367 & 0.0791 & 0.0779 & 0.0809 & 0.0678 & -0.0365 & 0.0201 & 0.1310 & 0.0697 & -0.0211 \\
\hline 3D38 - BCB L401 & 0.0935 & -0.1027 & -0.0489 & 0.1405 & -0.0757 & 0.0197 & 0.0062 & -0.0307 & 0.0177 & 0.0451 & -0.0356 & -0.0158 \\
\hline 3G7F - BCB L401 & 0.0894 & -0.0942 & -0.0919 & 0.1280 & -0.0635 & 0.0085 & 0.0003 & -0.0002 & -0.0080 & 0.0767 & -0.0360 & 0.0081 \\
\hline 3PRC - BCB L304 & -0.0004 & -0.0994 & -0.0496 & 0.1104 & 0.0011 & -0.0223 & 0.1516 & -0.0427 & -0.1187 & 0.1368 & 0.0735 & -0.0297 \\
\hline 5PRC - BCB L304 & 0.0080 & -0.1023 & -0.0336 & 0.1187 & -0.0006 & -0.0263 & 0.0221 & -0.0694 & -0.1630 & 0.1110 & 0.0877 & -0.0342 \\
\hline 6PRC - BCB L304 & 0.0054 & -0.0793 & -0.0703 & 0.1164 & -0.0044 & -0.0175 & 0.1005 & -0.0761 & -0.1558 & 0.0451 & 0.0492 & -0.0304 \\
\hline 7PRC - BCB L304 & 0.0223 & -0.0840 & -0.0782 & 0.1043 & 0.0101 & -0.0121 & 0.2388 & -0.0876 & -0.1552 & 0.0513 & -0.0976 & -0.0193 \\
\hline
\end{tabular}

Table 60: Extended conformational parameters and next-to-lowest energy basis normal-mode displacements of all BB cofactors from crystal structures of Blastochloris viridis.

\begin{tabular}{|c|c|c|c|c|c|c|c|c|c|c|c|c|}
\hline Cofactor ID & B2g_2 & B1g_2 & $\mathrm{Eu}(\mathrm{x}) \_2$ & $\mathrm{Eu}(\mathrm{y}) \_2$ & A1g_2 & A2g_2 & B2u_2 & B1u_2 & $A 2 u \_2$ & $\mathrm{Eg}(\mathrm{x}) \_2$ & $\mathrm{Eg}(\mathrm{y}) \_2$ & A1u_2 \\
\hline 1DXR - BCB M400 & 0.0299 & -0.0981 & -0.0522 & 0.1210 & -0.0055 & -0.0218 & 0.0890 & -0.0384 & -0.0210 & 0.1669 & 0.0748 & -0.0145 \\
\hline
\end{tabular}




\begin{tabular}{|c|c|c|c|c|c|c|c|c|c|c|c|c|}
\hline Cofactor ID & B2g_2 & B1g_2 & $\mathrm{Eu}(\mathrm{x}) \_2$ & $\mathrm{Eu}(\mathrm{y}) \_2$ & A1g_2 & A2g_2 & B2u_2 & B1u_2 & A2u_2 & $\mathrm{Eg}(\mathrm{x}) \_2$ & $\mathrm{Eg}(\mathrm{y}) \_2$ & A1u_2 \\
\hline 1PRC - BCB M601 & 0.1138 & -0.0514 & -0.1047 & 0.1146 & 0.0271 & 0.0192 & 0.0711 & 0.0215 & 0.0228 & 0.0967 & 0.0150 & 0.0254 \\
\hline 1R2C - BCB M1301 & 0.0303 & -0.1009 & -0.0227 & 0.0972 & 0.0017 & -0.0166 & 0.0834 & -0.1071 & -0.1521 & 0.2407 & -0.0615 & 0.0086 \\
\hline $1 \mathrm{VRN}$ - BCB M401 & 0.0198 & -0.1024 & -0.0488 & 0.1191 & -0.0044 & -0.0185 & 0.0663 & -0.0495 & -0.0007 & 0.2279 & 0.0832 & -0.0073 \\
\hline $215 N-B C B M 400$ & 0.0968 & -0.0904 & -0.0631 & 0.1504 & -0.0482 & 0.0171 & 0.0340 & 0.0307 & 0.0383 & 0.0781 & 0.0028 & 0.0280 \\
\hline 2JBL - BCB L1278 & 0.0153 & -0.1062 & -0.0386 & 0.1110 & 0.0003 & -0.0247 & 0.0187 & -0.0855 & -0.0800 & 0.2381 & 0.0468 & -0.0022 \\
\hline 2PRC - BCB M805 & 0.0134 & -0.0912 & -0.0428 & 0.1170 & -0.0011 & -0.0241 & 0.0478 & -0.0527 & -0.0634 & 0.2272 & -0.0046 & -0.0079 \\
\hline 2WJM - BCB M1324 & 0.0450 & 0.0818 & -0.0128 & 0.0617 & 0.0895 & 0.0600 & 0.0057 & -0.0427 & 0.0033 & 0.0948 & 0.0528 & -0.0077 \\
\hline 2WJN - BCB M1324 & 0.0426 & 0.0201 & -0.1015 & 0.0492 & 0.0512 & 0.0751 & 0.0436 & -0.0456 & 0.0029 & 0.1183 & 0.0752 & -0.0142 \\
\hline 3D38 - BCB M400 & 0.1700 & -0.0814 & -0.0806 & 0.0974 & -0.0583 & 0.0266 & 0.0213 & -0.0187 & 0.0338 & 0.0433 & 0.0043 & -0.0003 \\
\hline $3 G 7 F-B C B$ M400 & 0.1420 & -0.0707 & -0.0638 & 0.1769 & -0.0470 & 0.0115 & -0.0069 & 0.0629 & 0.0308 & 0.0939 & 0.0072 & 0.0520 \\
\hline 3PRC - BCB M805 & 0.0008 & -0.0885 & -0.0282 & 0.1255 & -0.0067 & -0.0081 & 0.0237 & -0.0473 & -0.0887 & 0.1990 & -0.0936 & -0.0041 \\
\hline 5PRC - BCB M805 & 0.0369 & -0.0947 & -0.0241 & 0.1239 & -0.0013 & -0.0127 & 0.0143 & -0.0364 & 0.0233 & 0.3487 & 0.0177 & -0.0005 \\
\hline $6 P R C-B C B$ M805 & 0.0301 & -0.0910 & -0.0102 & 0.1274 & -0.0030 & -0.0162 & 0.0120 & -0.0461 & -0.0585 & 0.3345 & -0.0102 & -0.0163 \\
\hline 7PRC - BCB M805 & 0.0168 & -0.0961 & -0.0035 & 0.1071 & -0.0044 & -0.0065 & 0.0429 & -0.0640 & -0.0081 & 0.2846 & -0.1077 & 0.0116 \\
\hline
\end{tabular}

Table 61: Extended conformational parameters and next-to-lowest energy basis normal-mode displacements of all DL cofactors from crystal structures of Blastochloris viridis.

\begin{tabular}{|c|c|c|c|c|c|c|c|c|c|c|c|c|}
\hline Cofactor ID & B2g_2 & B1g_2 & $\mathrm{Eu}(\mathrm{x}) \_2$ & $\mathrm{Eu}(\mathrm{y}) \_2$ & A1g_2 & A2g_2 & B2u_2 & B1u_2 & A2u_2 & $\mathrm{Eg}(\mathrm{x}) \_2$ & $E g(y) \_2$ & A1u_2 \\
\hline 1DXR - BCB L400 & -0.0544 & -0.1020 & -0.0404 & 0.1367 & 0.0015 & -0.0101 & 0.0734 & 0.1405 & -0.0167 & -0.0929 & -0.0251 & 0.0918 \\
\hline 1PRC - BCB L602 & -0.0451 & -0.0484 & -0.0125 & 0.1754 & 0.0515 & 0.0107 & -0.0282 & 0.1784 & -0.0085 & 0.0244 & -0.0219 & 0.1378 \\
\hline 1R2C - BCB L302 & -0.0362 & -0.1000 & -0.0543 & 0.1165 & 0.0312 & -0.0013 & 0.0613 & 0.0747 & 0.0986 & -0.0217 & 0.1238 & 0.0470 \\
\hline 1VRN - BCB L402 & 0.0041 & -0.0942 & -0.0547 & 0.1215 & 0.0180 & 0.0015 & 0.0632 & 0.1381 & -0.0070 & -0.1026 & -0.0410 & 0.1055 \\
\hline $215 N-B C B ~ L 400$ & 0.0652 & -0.0922 & -0.1105 & 0.1363 & -0.0285 & 0.0193 & 0.1094 & 0.1414 & 0.0296 & -0.0930 & 0.0457 & 0.1751 \\
\hline 2JBL - BCB L1274 & 0.0012 & -0.0910 & -0.0478 & 0.1230 & 0.0256 & 0.0005 & 0.0006 & 0.1179 & -0.0068 & -0.0079 & -0.0613 & 0.0814 \\
\hline 2PRC - BCB L302 & -0.0211 & -0.1098 & -0.0402 & 0.1429 & 0.0208 & 0.0026 & -0.0465 & 0.1065 & -0.0860 & -0.0532 & -0.1665 & 0.0787 \\
\hline 2WJM - BCB L1274 & -0.0128 & 0.0484 & -0.0440 & 0.1062 & 0.1196 & 0.0573 & 0.0197 & 0.1451 & 0.0102 & -0.0803 & -0.0349 & 0.0951 \\
\hline 2WJN - BCB L1274 & -0.0172 & 0.0336 & -0.0634 & 0.1488 & 0.1142 & 0.1161 & 0.0647 & 0.1687 & 0.0355 & -0.0815 & -0.0306 & 0.1266 \\
\hline 3D38 - BCB L400 & 0.1055 & -0.0831 & -0.1230 & 0.1478 & -0.0534 & 0.0313 & 0.1257 & 0.0825 & 0.0565 & -0.1222 & 0.0559 & 0.1172 \\
\hline 3G7F - BCB L400 & 0.0285 & -0.1014 & -0.0691 & 0.1216 & -0.0052 & 0.0205 & 0.0876 & 0.0599 & 0.0128 & -0.0935 & 0.0493 & 0.0990 \\
\hline 3PRC - BCB L302 & -0.0261 & -0.1064 & -0.0482 & 0.1292 & 0.0115 & -0.0016 & 0.0400 & 0.1337 & -0.0346 & -0.1206 & -0.0457 & 0.0805 \\
\hline 5PRC - BCB L302 & 0.0069 & -0.1149 & -0.0477 & 0.1027 & 0.0112 & -0.0005 & -0.0132 & 0.1465 & 0.0145 & -0.0097 & 0.0257 & 0.0847 \\
\hline 6PRC - BCB L302 & 0.0018 & -0.0985 & -0.0549 & 0.1299 & 0.0039 & 0.0035 & 0.0046 & 0.1561 & -0.0059 & -0.0432 & -0.0114 & 0.0610 \\
\hline 7PRC - BCB L302 & 0.0412 & -0.0936 & -0.0143 & 0.1323 & 0.0213 & -0.0006 & -0.0443 & 0.1371 & -0.0217 & -0.0012 & -0.0726 & 0.0839 \\
\hline
\end{tabular}

Table 62: Extended conformational parameters and next-to-lowest energy basis normal-mode displacements of all DM cofactors from crystal structures of Blastochloris viridis.

\begin{tabular}{|c|c|c|c|c|c|c|c|c|c|c|c|c|}
\hline Cofactor ID & B2g_2 & B1g_2 & $\mathrm{Eu}(\mathrm{x}) \_2$ & $\mathrm{Eu}(\mathrm{y}) \_2$ & A1g_2 & A2g_2 & B2u_2 & B1u_2 & A2u_2 & $E g(x) \_2$ & Eg(y)_2 & A1u_2 \\
\hline 1DXR - BCB M401 & -0.0100 & -0.0850 & -0.0538 & 0.1410 & 0.0125 & -0.0135 & 0.0396 & 0.1353 & 0.0384 & -0.0359 & 0.0339 & 0.0894 \\
\hline 1PRC - BCB M603 & -0.0778 & -0.0237 & -0.1323 & 0.1494 & 0.0771 & 0.0050 & -0.0004 & 0.2313 & 0.0537 & 0.0206 & -0.0252 & 0.1618 \\
\hline 1R2C - BCB M1303 & 0.0185 & -0.0817 & -0.0230 & 0.1081 & 0.0563 & -0.0076 & -0.0311 & 0.1381 & 0.0542 & 0.0667 & -0.0381 & 0.1098 \\
\hline 1VRN - BCB M403 & 0.0090 & -0.0760 & -0.0628 & 0.1398 & 0.0515 & -0.0138 & 0.0068 & 0.1453 & -0.0020 & -0.0703 & 0.0295 & 0.1227 \\
\hline
\end{tabular}




\begin{tabular}{|c|c|c|c|c|c|c|c|c|c|c|c|c|}
\hline Cofactor ID & B2g_2 & B1g_2 & $E u(x) \_2$ & Eu(y)_2 & A1g_2 & A2g_2 & B2u_2 & B1u_2 & A2u_2 & $\mathrm{Eg}(\mathrm{x}) \_2$ & Eg(y)_2 & A1u_2 \\
\hline $215 N-B C B$ M401 & 0.0778 & -0.0699 & -0.1226 & 0.1690 & -0.0017 & 0.0188 & 0.0826 & 0.1676 & 0.0345 & -0.0664 & 0.0817 & 0.1653 \\
\hline 2JBL - BCB M1324 & 0.0236 & -0.0907 & -0.0284 & 0.1254 & 0.0520 & -0.0094 & -0.0042 & 0.0933 & 0.0254 & -0.0280 & 0.0555 & 0.0921 \\
\hline 2PRC - BCB M806 & -0.0004 & -0.0945 & -0.0261 & 0.1107 & 0.0556 & -0.0074 & -0.0329 & 0.1316 & -0.0030 & -0.0541 & -0.0028 & 0.0585 \\
\hline 2WJM - BCB M1325 & -0.0236 & 0.0704 & -0.0251 & 0.1222 & 0.1487 & 0.0584 & 0.0259 & 0.2096 & -0.0185 & -0.1056 & 0.0872 & 0.1577 \\
\hline 2WJN - BCB M1325 & -0.0250 & 0.0462 & -0.0626 & 0.0983 & 0.1461 & 0.0922 & 0.0164 & 0.1889 & 0.0196 & -0.0985 & 0.0684 & 0.1682 \\
\hline 3D38 - BCB M401 & 0.0693 & -0.0839 & -0.0769 & 0.1307 & -0.0420 & 0.0205 & 0.1074 & 0.1495 & 0.0140 & -0.0861 & 0.0521 & 0.1852 \\
\hline $3 P R C-B C B$ M806 & 0.0046 & -0.0978 & -0.0282 & 0.1231 & 0.0338 & -0.0034 & 0.0357 & 0.1582 & 0.0325 & -0.0413 & -0.0157 & 0.0768 \\
\hline 5PRC - BCB M806 & 0.0412 & -0.0907 & 0.0035 & 0.1418 & 0.0287 & -0.0136 & 0.0342 & 0.1361 & 0.0278 & -0.0100 & -0.0022 & 0.0684 \\
\hline $6 P R C-B C B$ M806 & 0.0082 & -0.0759 & -0.0180 & 0.1576 & 0.0422 & -0.0074 & 0.0117 & 0.1606 & -0.0326 & -0.0148 & 0.0318 & 0.0975 \\
\hline 7PRC - BCB M806 & -0.0230 & -0.0951 & -0.0285 & 0.1487 & 0.0466 & -0.0102 & 0.0152 & 0.1137 & -0.1172 & 0.0936 & -0.1424 & 0.0524 \\
\hline
\end{tabular}

8.3 Rhodobacter sphaeroides bacteriopheophytin cofactor NSDs

Table 63: Minimum basis normal-mode displacements and conformational parameters of all ФA cofactors from crystal structures of Rhodobacter sphaeroides.

\begin{tabular}{|c|c|c|c|c|c|c|c|c|c|c|c|c|c|c|c|c|}
\hline Cofactor ID & Dip & Sip & B2g & B1g & $\mathrm{Eu}(\mathrm{x})$ & $\mathrm{Eu}(\mathrm{y})$ & A1g & $\mathrm{A} 2 \mathrm{~g}$ & Doop & Soop & $\mathrm{B} 2 \mathrm{u}$ & B1u & $\mathrm{A} 2 \mathrm{u}$ & $\mathrm{Eg}(\mathrm{x})$ & $\mathrm{Eg}(\mathrm{y})$ & A1u \\
\hline 1AIG - BPH L285 & 0.4884 & 0.0528 & -0.1092 & -0.2954 & -0.1178 & -0.0998 & 0.3392 & -0.0208 & 0.3981 & 0.0239 & 0.0879 & 0.2889 & 0.1891 & 0.0419 & -0.1721 & 0.0151 \\
\hline 1AIG - BPH N285 & 0.4382 & 0.0540 & -0.0240 & -0.3856 & -0.1054 & 0.0097 & 0.1754 & -0.0273 & 0.3523 & 0.0212 & -0.0020 & 0.2709 & 0.1849 & -0.0190 & -0.1270 & 0.0068 \\
\hline 1AIJ - BPH L285 & 0.3360 & 0.0564 & 0.0283 & -0.2012 & -0.0527 & -0.1369 & 0.2213 & -0.0339 & 0.5337 & 0.0159 & -0.0208 & 0.4973 & 0.1379 & 0.0169 & 0.1095 & 0.0763 \\
\hline 1AIJ - BPH R284 & 0.2221 & 0.0573 & 0.0066 & -0.1490 & -0.0355 & -0.0667 & 0.1394 & -0.0442 & 0.4808 & 0.0098 & -0.0538 & 0.4696 & -0.0552 & -0.0213 & 0.0459 & 0.0463 \\
\hline 1DS8 - BPH L1006 & 0.5479 & 0.0536 & 0.1067 & -0.3558 & -0.1224 & -0.0541 & 0.3772 & -0.0441 & 0.4603 & 0.0257 & -0.1218 & 0.4170 & 0.0668 & -0.0154 & -0.0367 & 0.1308 \\
\hline 1DS8 - BPH R2006 & 0.3976 & 0.0580 & 0.0174 & -0.2860 & -0.0812 & -0.0121 & 0.2610 & -0.0346 & 0.3726 & 0.0171 & -0.1286 & 0.3208 & -0.0241 & -0.1033 & -0.0532 & 0.0731 \\
\hline 1DV3 - BPH L1006 & 0.6311 & 0.0516 & 0.1952 & -0.3608 & -0.0854 & -0.0882 & 0.4579 & -0.0722 & 0.5521 & 0.0193 & -0.0304 & 0.5098 & 0.1507 & -0.0073 & 0.0032 & 0.1456 \\
\hline 1DV3 - BPH R2006 & 0.4072 & 0.0612 & -0.0131 & -0.2271 & -0.1329 & -0.0261 & 0.3078 & -0.0303 & 0.3342 & 0.0161 & -0.0595 & 0.2927 & 0.0689 & -0.0889 & -0.0720 & 0.0681 \\
\hline 1DV6 - BPH L1006 & 0.5299 & 0.0530 & 0.2150 & -0.2609 & -0.0218 & -0.1688 & 0.3650 & -0.0659 & 0.5638 & 0.0152 & -0.0520 & 0.4873 & 0.2364 & 0.0441 & -0.0057 & 0.1409 \\
\hline 1DV6 - BPH R2006 & 0.4297 & 0.0564 & -0.0797 & -0.2765 & -0.1025 & -0.0174 & 0.2992 & -0.0394 & 0.3698 & 0.0118 & -0.0138 & 0.3553 & 0.0433 & 0.0048 & -0.0448 & 0.0800 \\
\hline 1E14 - BPH C401 & 0.6322 & 0.0524 & 0.3910 & -0.4283 & -0.0098 & -0.0479 & 0.2383 & -0.0650 & 0.3607 & 0.0163 & -0.1045 & 0.2664 & 0.1319 & 0.0456 & -0.1618 & 0.0510 \\
\hline 1E6D - BPH C402 & 0.6319 & 0.0496 & 0.3728 & -0.3596 & -0.0631 & -0.0801 & 0.3409 & -0.0657 & 0.6228 & 0.0158 & -0.0346 & 0.5801 & 0.2072 & -0.0393 & 0.0168 & 0.0741 \\
\hline 1EYS - BPH L606 & 0.3180 & 0.0465 & -0.0287 & -0.1243 & -0.1064 & -0.1313 & 0.2282 & -0.0645 & 0.5690 & 0.0368 & -0.1249 & 0.4716 & 0.1020 & -0.1726 & -0.0768 & 0.1992 \\
\hline 1F6N - BPH L402 & 0.5206 & 0.0471 & 0.0973 & -0.3361 & -0.0531 & -0.0686 & 0.3683 & -0.0735 & 0.5027 & 0.0483 & -0.0875 & 0.4274 & 0.2325 & -0.0732 & -0.0319 & 0.0439 \\
\hline 1FNP - BPH L402 & 0.5116 & 0.0475 & 0.0788 & -0.3343 & -0.0642 & -0.0913 & 0.3554 & -0.0709 & 0.5875 & 0.0489 & -0.1941 & 0.4834 & 0.2659 & -0.0315 & -0.0240 & 0.0397 \\
\hline 1FNQ - BPH L402 & 0.5491 & 0.0447 & 0.1409 & -0.3360 & -0.0442 & -0.1126 & 0.3868 & -0.0677 & 0.5648 & 0.0468 & -0.0640 & 0.4998 & 0.2456 & -0.0349 & -0.0042 & 0.0590 \\
\hline 1JGW - BPH L855 & 0.5334 & 0.0601 & 0.1595 & -0.3232 & -0.0773 & -0.1054 & 0.3708 & -0.0033 & 0.1376 & 0.0039 & -0.0926 & 0.0986 & -0.0044 & -0.0129 & -0.0126 & 0.0172 \\
\hline 1JGX - BPH L855 & 0.6901 & 0.0615 & 0.2718 & -0.4386 & -0.0883 & -0.1250 & 0.4320 & 0.0056 & 0.1322 & 0.0041 & -0.0615 & 0.1104 & 0.0296 & -0.0019 & 0.0037 & 0.0246 \\
\hline 1JGY - BPH L855 & 0.5503 & 0.0639 & 0.1226 & -0.3298 & -0.0780 & -0.1087 & 0.4014 & 0.0076 & 0.1137 & 0.0031 & -0.0983 & 0.0277 & 0.0451 & 0.0089 & 0.0023 & 0.0196 \\
\hline 1JGZ - BPH L855 & 0.6407 & 0.0640 & 0.2405 & -0.4085 & -0.0967 & -0.1277 & 0.4000 & -0.0124 & 0.1313 & 0.0041 & -0.0745 & 0.0991 & 0.0317 & 0.0075 & 0.0112 & 0.0261 \\
\hline 1JHO - BPH L855 & 0.4852 & 0.0613 & 0.1679 & -0.3072 & -0.0736 & -0.1096 & 0.3087 & -0.0123 & 0.0714 & 0.0045 & -0.0253 & 0.0572 & -0.0087 & -0.0227 & -0.0216 & 0.0115 \\
\hline 1K6L - BPH L402 & 0.6155 & 0.0511 & 0.3574 & -0.3546 & -0.0652 & -0.0714 & 0.3344 & -0.0647 & 0.5802 & 0.0246 & 0.0494 & 0.5182 & 0.2346 & 0.0294 & -0.0110 & 0.0982 \\
\hline 1K6N - BPH L402 & 0.6153 & 0.0511 & 0.3574 & -0.3542 & -0.0651 & -0.0717 & 0.3344 & -0.0650 & 0.5800 & 0.0245 & 0.0498 & 0.5178 & 0.2346 & 0.0305 & -0.0114 & 0.0982 \\
\hline 1KBY - BPH L855 & 0.5784 & 0.0632 & 0.2232 & -0.3161 & -0.0475 & -0.1113 & 0.4125 & -0.0030 & 0.1673 & 0.0051 & -0.0997 & 0.1284 & -0.0141 & -0.0172 & -0.0263 & 0.0196 \\
\hline 1L9B - BPH M1006 & 0.6199 & 0.0567 & 0.2271 & -0.3097 & -0.0508 & -0.0500 & 0.4807 & -0.0249 & 0.1966 & 0.0038 & -0.1458 & 0.1250 & 0.0368 & -0.0168 & -0.0039 & 0.0101 \\
\hline 1L9J - BPH M1006 & 0.5620 & 0.0560 & 0.1771 & -0.3143 & -0.0640 & -0.0450 & 0.4230 & -0.0266 & 0.1074 & 0.0026 & -0.0660 & 0.0834 & -0.0017 & -0.0094 & 0.0045 & 0.0107 \\
\hline
\end{tabular}




\begin{tabular}{|c|c|c|c|c|c|c|c|c|c|c|c|c|c|c|c|c|}
\hline 1L9J - BPH S2006 & 0.6332 & 0.0560 & 0.1443 & -0.3892 & -0.0581 & -0.0551 & 0.4707 & -0.0267 & 0.1484 & 0.0023 & -0.0694 & 0.1288 & 0.0012 & -0.0232 & -0.0031 & 0.0073 \\
\hline 1M3X - BPH L855 & 0.5807 & 0.0613 & 0.2665 & -0.3141 & -0.0731 & -0.0751 & 0.3956 & -0.0083 & 0.1642 & 0.0047 & -0.1042 & 0.1242 & 0.0031 & -0.0127 & -0.0131 & 0.0182 \\
\hline 1MPS - BPH L402 & 0.5143 & 0.0466 & 0.1175 & -0.3502 & -0.0253 & -0.0827 & 0.3404 & -0.0686 & 0.4977 & 0.0359 & -0.0899 & 0.4663 & 0.1259 & -0.0032 & 0.0423 & 0.0675 \\
\hline 10GV - BPH L1284 & 0.6081 & 0.0533 & 0.3247 & -0.3004 & -0.0968 & -0.0486 & 0.4028 & -0.0121 & 0.2269 & 0.0051 & -0.0165 & 0.2030 & 0.0967 & -0.0197 & -0.0100 & 0.0126 \\
\hline 1PCR - BPH L402 & 0.5072 & 0.0446 & -0.0766 & -0.3733 & -0.0447 & -0.0899 & 0.3117 & -0.0695 & 0.6375 & 0.0622 & -0.0288 & 0.5427 & 0.2831 & 0.0568 & -0.1444 & 0.0822 \\
\hline 1PSS - BPH L271 & 0.3879 & 0.0492 & -0.0767 & -0.3617 & -0.0128 & -0.0509 & 0.0931 & -0.0484 & 0.7109 & 0.0318 & 0.1138 & 0.6605 & 0.1477 & 0.1151 & -0.1404 & 0.0355 \\
\hline 1PST - BPH L271 & 0.4356 & 0.0529 & -0.1588 & -0.3238 & -0.0503 & -0.0440 & 0.2311 & -0.0425 & 0.4625 & 0.0195 & 0.1139 & 0.3345 & 0.2822 & 0.0611 & -0.0607 & 0.0443 \\
\hline 1PYH - BPH B402 & 0.4077 & 0.0538 & 0.1947 & -0.2791 & -0.0825 & -0.0674 & 0.1655 & -0.1083 & 0.5344 & 0.0275 & 0.0122 & 0.4690 & 0.1952 & 0.0172 & -0.0204 & 0.1634 \\
\hline 1QOV - BPH M1402 & 0.6158 & 0.0509 & 0.3575 & -0.3552 & -0.0644 & -0.0715 & 0.3342 & -0.0654 & 0.5808 & 0.0246 & 0.0488 & 0.5191 & 0.2339 & 0.0292 & -0.0109 & 0.0987 \\
\hline 1RG5 - BPH L402 & 0.6140 & 0.0584 & 0.1220 & -0.4189 & -0.0796 & -0.0520 & 0.4060 & -0.1132 & 0.5603 & 0.0242 & -0.0677 & 0.5084 & 0.1651 & -0.0563 & -0.0141 & 0.1424 \\
\hline 1RGN - BPH L402 & 0.6515 & 0.0721 & 0.2400 & -0.4500 & -0.0972 & -0.1061 & 0.3642 & -0.1052 & 0.6317 & 0.0292 & 0.1323 & 0.5175 & 0.2671 & -0.1324 & 0.0717 & 0.1406 \\
\hline 1RQK - BPH L402 & 0.5809 & 0.0610 & 0.2082 & -0.3866 & -0.0714 & -0.0341 & 0.3605 & -0.0914 & 0.5046 & 0.0226 & -0.0124 & 0.4393 & 0.1971 & -0.0597 & -0.0056 & 0.1380 \\
\hline 1RZZ - BPH R2006 & 0.6731 & 0.0584 & 0.3016 & -0.3238 & -0.0792 & -0.0699 & 0.4960 & -0.0109 & 0.1836 & 0.0050 & -0.0021 & 0.1620 & 0.0737 & -0.0121 & 0.0400 & 0.0161 \\
\hline 1S00 - BPH R2006 & 0.6650 & 0.0564 & 0.3539 & -0.3800 & -0.0659 & -0.0298 & 0.4089 & -0.0153 & 0.2018 & 0.0049 & 0.0119 & 0.1879 & 0.0636 & -0.0078 & 0.0276 & 0.0201 \\
\hline 1YST - BPH L276 & 0.4536 & 0.0707 & 0.0966 & -0.3500 & -0.1722 & -0.1621 & 0.0794 & -0.1081 & 0.9179 & 0.0235 & -0.8485 & 0.2982 & 0.1157 & -0.0938 & -0.0968 & 0.0461 \\
\hline 1Z9J - BPH A855 & 0.7012 & 0.0574 & 0.1766 & -0.5748 & -0.0886 & -0.0327 & 0.3472 & -0.0236 & 0.1599 & 0.0020 & 0.1436 & -0.0524 & 0.0448 & 0.0048 & 0.0096 & 0.0098 \\
\hline 1Z9K - BPH A855 & 0.5509 & 0.0638 & -0.1422 & 0.3846 & 0.0698 & -0.1844 & 0.3105 & -0.0067 & 0.0808 & 0.0035 & 0.0608 & 0.0415 & 0.0204 & 0.0196 & -0.0173 & 0.0029 \\
\hline 2BNP - BH1 A1284 & 0.8103 & 0.0637 & 0.5344 & -0.5624 & -0.1597 & -0.0498 & 0.1318 & -0.0962 & 0.6204 & 0.0427 & -0.1010 & 0.5215 & 0.1244 & 0.0375 & -0.0807 & 0.2815 \\
\hline 2BNS - BH1 A1284 & 0.6335 & 0.0608 & 0.2431 & -0.5288 & -0.1446 & -0.0832 & 0.1540 & -0.1052 & 0.6253 & 0.0433 & -0.0526 & 0.5379 & 0.1048 & -0.0381 & -0.0741 & 0.2844 \\
\hline 2BOZ - BH1 L1284 & 0.5774 & 0.0583 & 0.3171 & -0.3097 & -0.1560 & -0.0767 & 0.3032 & -0.1214 & 0.5300 & 0.0218 & 0.0101 & 0.4588 & 0.2327 & -0.0088 & -0.0305 & 0.1233 \\
\hline 2GMR - BPH L305 & 0.4917 & 0.0429 & 0.1690 & -0.2697 & -0.1193 & -0.0501 & 0.3417 & -0.0836 & 0.5610 & 0.0460 & 0.0491 & 0.4600 & 0.2088 & 0.0539 & -0.0378 & 0.2299 \\
\hline 2GNU - BPH M1284 & 0.7457 & 0.0534 & 0.5193 & -0.4235 & -0.1196 & -0.0527 & 0.2527 & -0.1615 & 0.6376 & 0.0434 & -0.0116 & 0.5357 & 0.1502 & -0.0626 & -0.1036 & 0.2866 \\
\hline 2HG3 - BPH L402 & 0.6095 & 0.0528 & 0.1962 & -0.4010 & -0.0555 & -0.1332 & 0.3746 & -0.1051 & 0.5755 & 0.0267 & 0.0914 & 0.4761 & 0.2630 & -0.0385 & -0.0564 & 0.1495 \\
\hline 2HG9 - BPH L402 & 0.5530 & 0.0587 & 0.1865 & -0.3979 & -0.0985 & -0.0894 & 0.2942 & -0.0922 & 0.5238 & 0.0266 & 0.0526 & 0.4644 & 0.1763 & -0.0825 & -0.0198 & 0.1329 \\
\hline 2HH1 - BPH L402 & 0.5694 & 0.0490 & 0.2537 & -0.3855 & -0.1016 & -0.1572 & 0.2655 & -0.0756 & 0.6199 & 0.0244 & 0.0035 & 0.5685 & 0.1320 & -0.1307 & -0.0714 & 0.1465 \\
\hline 2HHK - BPH L402 & 0.5832 & 0.0548 & 0.2444 & -0.3391 & -0.0726 & -0.1109 & 0.3725 & -0.0953 & 0.5718 & 0.0295 & 0.0540 & 0.4982 & 0.2153 & -0.1102 & -0.0473 & 0.1230 \\
\hline 2HIT - BPH L402 & 0.6377 & 0.0487 & -0.0498 & -0.4170 & -0.1015 & -0.1531 & 0.4346 & -0.0875 & 0.6360 & 0.0361 & 0.1505 & 0.4570 & 0.3195 & -0.2030 & 0.0838 & 0.1507 \\
\hline $2 \mathrm{HJ} 6-\mathrm{BPH}$ L402 & 0.4891 & 0.0621 & -0.1414 & -0.1610 & -0.0236 & -0.1559 & 0.3877 & -0.1348 & 0.4928 & 0.0246 & -0.0187 & 0.2957 & 0.2459 & -0.2727 & 0.0427 & 0.1355 \\
\hline 2J8C - BPH L1286 & 0.6192 & 0.0556 & 0.3542 & -0.3139 & -0.1635 & -0.1151 & 0.3309 & -0.0998 & 0.5735 & 0.0595 & -0.0437 & 0.3648 & 0.2629 & -0.1975 & -0.0677 & 0.2850 \\
\hline 2J8D - BPH L1287 & 0.6544 & 0.0580 & 0.3989 & -0.3041 & -0.1733 & -0.1256 & 0.3359 & -0.1344 & 0.6162 & 0.0627 & 0.0534 & 0.4652 & 0.2343 & -0.1475 & -0.1158 & 0.2654 \\
\hline $2 J I Y-B P H ~ L 1285$ & 0.5703 & 0.0516 & 0.2941 & -0.3051 & -0.1449 & -0.1025 & 0.3242 & -0.0952 & 0.5500 & 0.0266 & -0.0112 & 0.4669 & 0.2401 & -0.0800 & -0.0003 & 0.1427 \\
\hline 2JJO - BPH L1285 & 0.5479 & 0.0589 & 0.1755 & -0.3900 & -0.0991 & -0.1247 & 0.2912 & -0.0841 & 0.5288 & 0.0278 & 0.1136 & 0.4209 & 0.2505 & -0.0236 & 0.1003 & 0.1269 \\
\hline 2RCR - BPH L550 & 0.3669 & 0.0488 & -0.1225 & -0.2357 & -0.1000 & -0.0239 & 0.2007 & -0.1148 & 0.4431 & 0.0091 & 0.2890 & 0.2695 & 0.0973 & -0.0655 & -0.1617 & -0.0165 \\
\hline 2UWS - BPH L1284 & 0.7749 & 0.0772 & 0.2606 & -0.5799 & -0.0725 & -0.0724 & 0.4119 & -0.1270 & 0.6226 & 0.0925 & 0.4440 & 0.3452 & 0.0993 & -0.0597 & -0.1899 & 0.1479 \\
\hline 2UWT - BPH L1284 & 0.8073 & 0.0622 & 0.3718 & -0.5793 & -0.1326 & -0.0503 & 0.3820 & -0.1091 & 0.6540 & 0.0569 & 0.1462 & 0.4787 & 0.3472 & 0.0045 & 0.0332 & 0.2354 \\
\hline 2UWU - BPH L1286 & 0.6345 & 0.0592 & 0.2670 & -0.3772 & -0.1640 & -0.1275 & 0.3687 & -0.0997 & 0.6783 & 0.0544 & 0.1306 & 0.5125 & 0.3039 & 0.0007 & -0.0708 & 0.2881 \\
\hline 2UWV - BPH L1284 & 0.6543 & 0.0596 & 0.2867 & -0.3964 & -0.1378 & -0.1137 & 0.3807 & -0.1096 & 0.6360 & 0.0582 & 0.0835 & 0.5196 & 0.2417 & -0.0286 & -0.0815 & 0.2483 \\
\hline 2UWW - BPH L1288 & 0.6468 & 0.0558 & 0.2903 & -0.3439 & -0.1497 & -0.1112 & 0.4058 & -0.1276 & 0.6604 & 0.0582 & 0.1179 & 0.5151 & 0.2730 & -0.0332 & -0.1109 & 0.2627 \\
\hline 2UX3 - BPH L1284 & 0.6441 & 0.0652 & 0.2699 & -0.4030 & -0.1237 & -0.0648 & 0.3868 & -0.1025 & 0.6268 & 0.0773 & 0.0766 & 0.5421 & 0.2588 & 0.0279 & -0.1094 & 0.1158 \\
\hline $2 U X 4$ - BPH L1286 & 0.7019 & 0.0665 & 0.2938 & -0.4614 & -0.1241 & -0.0645 & 0.4017 & -0.1116 & 0.5144 & 0.0763 & 0.0042 & 0.2670 & 0.3336 & -0.0671 & 0.0411 & 0.2754 \\
\hline 2UX5 - BPH L1285 & 0.7401 & 0.0549 & 0.3620 & -0.4835 & -0.1689 & -0.1273 & 0.3595 & -0.0950 & 0.7209 & 0.0570 & 0.1141 & 0.6134 & 0.2379 & -0.0661 & -0.1394 & 0.2236 \\
\hline 2UXJ - BPH L1285 & 0.7545 & 0.0565 & 0.4055 & -0.4360 & -0.2077 & -0.1403 & 0.3825 & -0.0756 & 0.5690 & 0.0866 & -0.0275 & 0.3166 & 0.1925 & 0.0070 & 0.1196 & 0.4140 \\
\hline 2UXK - BPH L1287 & 0.6651 & 0.0593 & 0.3241 & -0.3421 & -0.1693 & -0.1238 & 0.4047 & -0.1122 & 0.5058 & 0.0823 & 0.0598 & 0.3589 & 0.2099 & -0.0367 & 0.0041 & 0.2794 \\
\hline 2UXL - BPH L1283 & 0.6053 & 0.0633 & 0.2613 & -0.4149 & -0.0610 & -0.1043 & 0.3139 & -0.1131 & 0.5534 & 0.1106 & -0.0249 & 0.3024 & 0.2197 & -0.2168 & 0.0744 & 0.3367 \\
\hline
\end{tabular}




\begin{tabular}{|c|c|c|c|c|c|c|c|c|c|c|c|c|c|c|c|c|}
\hline 2UXM - BPH L1285 & 0.7443 & 0.0726 & 0.3517 & -0.4676 & -0.0529 & -0.1366 & 0.4195 & -0.1190 & 0.5163 & 0.0751 & 0.0644 & 0.3556 & 0.2482 & -0.1181 & 0.0667 & 0.2365 \\
\hline 2WX5 - BPH L1288 & 0.6179 & 0.0613 & 0.2321 & -0.4500 & -0.1528 & -0.0322 & 0.2907 & -0.1282 & 0.6917 & 0.0579 & 0.0312 & 0.4619 & 0.4238 & -0.0239 & 0.0116 & 0.2895 \\
\hline 3DSY - BPH L503 & 0.6154 & 0.0511 & 0.3574 & -0.3543 & -0.0654 & -0.0710 & 0.3344 & -0.0652 & 0.5802 & 0.0246 & 0.0495 & 0.5183 & 0.2344 & 0.0292 & -0.0111 & 0.0983 \\
\hline 3DTA - BPH L503 & 0.6161 & 0.0510 & 0.3581 & -0.3546 & -0.0653 & -0.0710 & 0.3347 & -0.0651 & 0.5803 & 0.0246 & 0.0496 & 0.5186 & 0.2343 & 0.0296 & -0.0113 & 0.0976 \\
\hline 3DTR - BPH L503 & 0.6155 & 0.0510 & 0.3576 & -0.3546 & -0.0649 & -0.0715 & 0.3342 & -0.0652 & 0.5803 & 0.0246 & 0.0493 & 0.5181 & 0.2348 & 0.0300 & -0.0104 & 0.0982 \\
\hline 3DTS - BPH M503 & 0.6155 & 0.0512 & 0.3574 & -0.3545 & -0.0651 & -0.0717 & 0.3345 & -0.0648 & 0.5802 & 0.0245 & 0.0494 & 0.5182 & 0.2346 & 0.0300 & -0.0114 & 0.0983 \\
\hline 3DU2 - BPH L503 & 0.6156 & 0.0511 & 0.3575 & -0.3549 & -0.0653 & -0.0711 & 0.3342 & -0.0645 & 0.5808 & 0.0245 & 0.0490 & 0.5187 & 0.2349 & 0.0295 & -0.0112 & 0.0985 \\
\hline 3DU3 - BPH L503 & 0.6152 & 0.0512 & 0.3574 & -0.3542 & -0.0652 & -0.0717 & 0.3340 & -0.0648 & 0.5800 & 0.0245 & 0.0496 & 0.5177 & 0.2349 & 0.0303 & -0.0111 & 0.0986 \\
\hline 3DUQ - BPH L503 & 0.6156 & 0.0512 & 0.3582 & -0.3541 & -0.0662 & -0.0717 & 0.3341 & -0.0647 & 0.5796 & 0.0245 & 0.0492 & 0.5175 & 0.2345 & 0.0301 & -0.0116 & 0.0987 \\
\hline 4RCR - BPH L284 & 0.9389 & 0.0734 & -0.0490 & 0.0605 & -0.3647 & 0.0652 & 0.8469 & -0.1449 & 0.5748 & 0.0656 & -0.3945 & 0.3542 & 0.1690 & 0.0892 & -0.1107 & 0.0212 \\
\hline
\end{tabular}

Table 64: Minimum basis normal-mode displacements and conformational parameters of all ФB cofactors from crystal structures of Rhodobacter sphaeroides.

\begin{tabular}{|c|c|c|c|c|c|c|c|c|c|c|c|c|c|c|c|c|}
\hline Cofactor ID & Dip & sip & B2g & B1g & $E u(x)$ & $E u(y)$ & A1g & A2g & Doop & doop & $B 2 u$ & B1u & $A 2 u$ & $\mathrm{Eg}(\mathrm{x})$ & $\mathrm{Eg}(\mathrm{y})$ & A1u \\
\hline 1AIG - BPH L284 & 0.5380 & 0.0523 & -0.0690 & -0.4624 & -0.1405 & -0.0401 & 0.2173 & -0.0478 & 0.3987 & 0.0140 & 0.1589 & 0.3008 & 0.1983 & -0.0307 & -0.0034 & 0.0542 \\
\hline $1 \mathrm{AIG}-\mathrm{BPH} 0310$ & 0.3845 & 0.0526 & 0.0921 & -0.3202 & 0.0242 & 0.0314 & 0.1715 & -0.0764 & 0.4820 & 0.0156 & 0.0902 & 0.4525 & 0.1283 & -0.0056 & 0.0076 & 0.0539 \\
\hline 1AIJ - BPH L284 & 0.3654 & 0.0556 & -0.0596 & -0.3055 & 0.0078 & -0.0822 & 0.1637 & -0.0546 & 0.3703 & 0.0080 & 0.0115 & 0.3204 & 0.1733 & -0.0299 & 0.0220 & 0.0544 \\
\hline 1AIJ - BPH S311 & 0.3484 & 0.0557 & -0.1869 & -0.2032 & -0.0215 & -0.0592 & 0.1907 & -0.0692 & 0.3831 & 0.0142 & 0.0646 & 0.3739 & 0.0144 & 0.0201 & -0.0101 & 0.0451 \\
\hline 1DS8 - BPH M1005 & 0.5662 & 0.0607 & 0.1405 & -0.4141 & -0.0660 & -0.0950 & 0.3375 & -0.0467 & 0.4376 & 0.0181 & 0.1610 & 0.3532 & 0.1540 & -0.0692 & -0.0695 & 0.0863 \\
\hline 1DS8 - BPH S2005 & 0.3448 & 0.0536 & -0.1976 & -0.1550 & -0.0078 & -0.0649 & 0.2160 & -0.0699 & 0.5905 & 0.0151 & 0.0728 & 0.5285 & 0.2290 & -0.0388 & 0.0389 & 0.0934 \\
\hline 1DV3 - BPH M1005 & 0.5338 & 0.0576 & -0.1520 & -0.3620 & -0.1279 & -0.1148 & 0.3163 & -0.0357 & 0.5405 & 0.0153 & 0.1275 & 0.4946 & 0.1332 & -0.0641 & -0.0106 & 0.0961 \\
\hline 1DV3 - BPH S2005 & 0.3525 & 0.0532 & 0.0568 & -0.2331 & -0.0037 & -0.0175 & 0.2453 & -0.0785 & 0.5335 & 0.0133 & 0.0712 & 0.5057 & 0.1097 & -0.0517 & 0.0564 & 0.0775 \\
\hline 1DV6 - BPH M1005 & 0.5502 & 0.0610 & -0.0732 & -0.3222 & -0.1091 & -0.1082 & 0.4089 & -0.0530 & 0.4441 & 0.0151 & 0.0698 & 0.3895 & 0.1479 & -0.0721 & 0.0121 & 0.1160 \\
\hline 1DV6 - BPH S2005 & 0.4118 & 0.0537 & -0.1748 & -0.2183 & -0.0738 & -0.0511 & 0.2764 & -0.0828 & 0.5122 & 0.0131 & 0.1121 & 0.4637 & 0.1553 & -0.0397 & 0.0705 & 0.0641 \\
\hline $1 \mathrm{E} 14$ - BPH C402 & 0.7554 & 0.0500 & 0.3309 & -0.5812 & -0.0791 & -0.0511 & 0.3370 & -0.0310 & 0.4218 & 0.0309 & 0.0976 & 0.3438 & 0.1915 & -0.0535 & -0.0381 & 0.0960 \\
\hline $1 E 6 D-B P H C 401$ & 0.7378 & 0.0510 & 0.5331 & -0.3359 & -0.0493 & -0.0820 & 0.3695 & -0.0396 & 0.7428 & 0.0270 & 0.2104 & 0.6128 & 0.2559 & -0.2022 & 0.1526 & 0.0481 \\
\hline 1EYS - BPH M605 & 0.3516 & 0.0515 & 0.0469 & -0.2230 & -0.1139 & -0.1044 & 0.1971 & -0.0945 & 0.4154 & 0.0384 & -0.0204 & 0.3501 & 0.1341 & 0.0124 & 0.0237 & 0.1759 \\
\hline $1 F 6 N$ - BPH M401 & 0.5088 & 0.0428 & 0.1135 & -0.3729 & -0.0406 & -0.0673 & 0.3057 & -0.0855 & 0.6229 & 0.0489 & 0.0073 & 0.5694 & 0.2204 & -0.1218 & -0.0063 & -0.0170 \\
\hline 1FNP - BPH M401 & 0.4813 & 0.0443 & 0.1241 & -0.3327 & -0.0546 & -0.0564 & 0.3044 & -0.0817 & 0.7131 & 0.0528 & 0.0756 & 0.5844 & 0.3844 & -0.0964 & 0.0598 & -0.0248 \\
\hline 1FNQ - BPH M401 & 0.4984 & 0.0432 & 0.1652 & -0.3484 & -0.0441 & -0.0641 & 0.2956 & -0.0795 & 0.6441 & 0.0478 & 0.0545 & 0.5454 & 0.3105 & -0.1222 & 0.0458 & -0.0319 \\
\hline 1JGW - BPH M854 & 0.6418 & 0.0656 & 0.1837 & -0.4463 & -0.0188 & -0.1077 & 0.4082 & -0.0190 & 0.1237 & 0.0035 & 0.0373 & 0.1125 & 0.0055 & -0.0314 & 0.0086 & 0.0129 \\
\hline 1JGX - BPH M854 & 0.6158 & 0.0662 & 0.1088 & -0.4810 & 0.0219 & -0.1222 & 0.3465 & -0.0243 & 0.1151 & 0.0040 & 0.0211 & 0.1039 & 0.0206 & -0.0278 & 0.0235 & 0.0155 \\
\hline 1JGZ - BPH M854 & 0.6298 & 0.0645 & 0.1679 & -0.4568 & -0.0825 & -0.1518 & 0.3602 & -0.0147 & 0.0986 & 0.0030 & -0.0115 & 0.0821 & 0.0287 & -0.0393 & 0.0194 & 0.0099 \\
\hline 1JHO - BPH M854 & 0.6097 & 0.0659 & 0.0563 & -0.4757 & -0.0750 & -0.0845 & 0.3592 & -0.0211 & 0.0905 & 0.0028 & -0.0424 & 0.0781 & -0.0015 & -0.0126 & 0.0067 & 0.0097 \\
\hline $1 \mathrm{~K} 6 \mathrm{~L}-\mathrm{BPH}$ M401 & 0.5899 & 0.0547 & 0.3118 & -0.3677 & -0.0499 & -0.0493 & 0.3320 & -0.0215 & 0.6392 & 0.0246 & 0.1635 & 0.5000 & 0.3283 & -0.1282 & 0.0314 & 0.0819 \\
\hline $1 \mathrm{~K} 6 \mathrm{~N}-\mathrm{BPH} \mathrm{M} 401$ & 0.5898 & 0.0546 & 0.3121 & -0.3676 & -0.0506 & -0.0493 & 0.3316 & -0.0213 & 0.6398 & 0.0246 & 0.1633 & 0.5008 & 0.3278 & -0.1290 & 0.0321 & 0.0824 \\
\hline $1 \mathrm{KBY}-\mathrm{BPH}$ L854 & 0.6727 & 0.0677 & 0.2925 & -0.3994 & -0.0173 & -0.1416 & 0.4321 & -0.0206 & 0.1293 & 0.0038 & 0.0520 & 0.1116 & -0.0069 & -0.0349 & 0.0130 & 0.0114 \\
\hline 1L9B - BPH L1005 & 0.6858 & 0.0563 & 0.3573 & -0.4019 & -0.0081 & -0.0577 & 0.4212 & -0.0180 & 0.1712 & 0.0051 & 0.0677 & 0.1310 & 0.0700 & -0.0471 & 0.0154 & 0.0148 \\
\hline 1L9J - BPH L1005 & 0.5871 & 0.0552 & 0.2445 & -0.3309 & -0.0273 & -0.0670 & 0.4120 & -0.0205 & 0.0549 & 0.0032 & -0.0489 & -0.0006 & 0.0055 & -0.0054 & -0.0224 & 0.0080 \\
\hline 1L9J - BPH R2005 & 0.6763 & 0.0559 & 0.3136 & -0.3168 & -0.0726 & -0.0816 & 0.4962 & -0.0226 & 0.0741 & 0.0019 & -0.0659 & 0.0113 & 0.0282 & -0.0116 & 0.0086 & 0.0043 \\
\hline 1M3X - ВРH M854 & 0.6359 & 0.0635 & 0.1857 & -0.4455 & -0.0104 & -0.1001 & 0.4010 & -0.0202 & 0.1328 & 0.0039 & 0.0279 & 0.1240 & 0.0012 & -0.0344 & 0.0095 & 0.0148 \\
\hline 1MPS - BPH L401 & 0.4692 & 0.0441 & 0.1733 & -0.2707 & -0.0852 & -0.0460 & 0.3194 & -0.0740 & 0.6327 & 0.0433 & 0.1006 & 0.4698 & 0.3158 & -0.2157 & 0.1501 & 0.0267 \\
\hline
\end{tabular}




\begin{tabular}{|c|c|c|c|c|c|c|c|c|c|c|c|c|c|c|c|c|}
\hline 10GV - BPH M1304 & 0.5610 & 0.0549 & 0.2360 & -0.3112 & -0.0353 & -0.0803 & 0.3921 & -0.0271 & 0.2003 & 0.0049 & 0.0615 & 0.1637 & 0.0929 & -0.0232 & 0.0163 & 0.0107 \\
\hline 1PCR - BPH M401 & 0.5480 & 0.0406 & 0.1459 & -0.4165 & -0.0902 & -0.0310 & 0.3003 & -0.0792 & 0.7094 & 0.0548 & -0.0333 & 0.6579 & 0.2569 & -0.0362 & 0.0435 & -0.0068 \\
\hline 1PSS - BPH M5 & 0.2978 & 0.0516 & -0.0611 & -0.2364 & -0.0865 & -0.0293 & 0.1371 & -0.0438 & 0.3235 & 0.0188 & 0.1485 & 0.1907 & 0.2075 & 0.0156 & 0.0374 & 0.0389 \\
\hline 1PST - BPH M5 & 0.3609 & 0.0540 & -0.1806 & -0.2330 & -0.0404 & -0.0452 & 0.1936 & -0.0469 & 0.3661 & 0.0106 & 0.0840 & 0.2952 & 0.1954 & 0.0085 & 0.0386 & 0.0096 \\
\hline 1PYH - BPH A401 & 0.4374 & 0.0630 & 0.2296 & -0.3381 & -0.0808 & -0.0476 & 0.1020 & -0.0717 & 0.5845 & 0.0245 & 0.0586 & 0.4901 & 0.2727 & -0.1107 & -0.0106 & 0.1061 \\
\hline 1QOV - BPH M1401 & 0.5905 & 0.0547 & 0.3117 & -0.3686 & -0.0500 & -0.0499 & 0.3319 & -0.0215 & 0.6391 & 0.0246 & 0.1633 & 0.5009 & 0.3275 & -0.1268 & 0.0304 & 0.0817 \\
\hline 1RG5 - BPH M401 & 0.6769 & 0.0560 & 0.1942 & -0.4047 & -0.0686 & -0.1334 & 0.4781 & -0.0755 & 0.5593 & 0.0238 & 0.0631 & 0.4887 & 0.2223 & -0.0855 & 0.0806 & 0.0821 \\
\hline 1RGN - BPH M401 & 0.6039 & 0.0549 & 0.1982 & -0.3315 & 0.0176 & -0.2374 & 0.3850 & -0.1030 & 0.4174 & 0.0165 & 0.0870 & 0.3445 & 0.0488 & -0.1852 & 0.0854 & 0.0628 \\
\hline 1RQK - BPH M401 & 0.7398 & 0.0510 & 0.2881 & -0.6020 & -0.0065 & -0.0515 & 0.2918 & -0.1183 & 0.4906 & 0.0243 & 0.0902 & 0.3451 & 0.1848 & -0.0519 & 0.2697 & 0.0621 \\
\hline 1RVJ - BPH M855 & 0.6429 & 0.0551 & 0.2554 & -0.3753 & -0.0555 & -0.0749 & 0.4447 & -0.0285 & 0.1366 & 0.0045 & -0.0397 & 0.1103 & 0.0580 & -0.0267 & -0.0086 & 0.0276 \\
\hline 1RY5 - BPH M855 & 0.6268 & 0.0553 & 0.2779 & -0.3632 & -0.0438 & -0.0590 & 0.4215 & -0.0254 & 0.1566 & 0.0035 & 0.0011 & 0.1410 & 0.0579 & -0.0330 & -0.0046 & 0.0137 \\
\hline 1RZH - BPH M855 & 0.6467 & 0.0547 & 0.3163 & -0.3685 & -0.0429 & -0.0722 & 0.4182 & -0.0230 & 0.1766 & 0.0041 & 0.0095 & 0.1558 & 0.0767 & -0.0268 & 0.0021 & 0.0138 \\
\hline 1RZZ - BPH M1005 & 0.5974 & 0.0558 & 0.3017 & -0.3119 & -0.0716 & -0.0562 & 0.3996 & -0.0242 & 0.1997 & 0.0041 & 0.0066 & 0.1795 & 0.0832 & -0.0184 & -0.0017 & 0.0190 \\
\hline 1RZZ - BPH S2005 & 0.5905 & 0.0550 & 0.2730 & -0.2787 & -0.0466 & -0.0653 & 0.4355 & -0.0199 & 0.1439 & 0.0030 & 0.0288 & 0.1307 & 0.0472 & -0.0175 & -0.0082 & 0.0139 \\
\hline 1S00 - BPH M1005 & 0.6231 & 0.0530 & 0.2716 & -0.4044 & -0.0941 & -0.0893 & 0.3651 & -0.0287 & 0.1747 & 0.0054 & -0.0108 & 0.1480 & 0.0882 & -0.0077 & -0.0146 & 0.0208 \\
\hline $1 \mathrm{~S} 00$ - BPH S2005 & 0.5427 & 0.0588 & 0.2571 & -0.2400 & -0.0392 & -0.0792 & 0.4033 & -0.0183 & 0.1764 & 0.0048 & 0.0439 & 0.1593 & 0.0544 & 0.0066 & 0.0228 & 0.0172 \\
\hline 1YF6 - BPH L855 & 0.6177 & 0.0652 & 0.0838 & -0.4400 & -0.0286 & -0.1324 & 0.3958 & -0.0768 & 0.5734 & 0.0481 & 0.2192 & 0.4566 & 0.1830 & -0.0514 & -0.1535 & 0.1121 \\
\hline 1YST - BPH M309 & 1.0367 & 0.0729 & -0.3481 & -0.7806 & -0.1850 & 0.1378 & 0.5319 & -0.0897 & 0.6158 & 0.0192 & -0.5323 & -0.2589 & 0.1182 & -0.0875 & -0.0659 & -0.0538 \\
\hline 1Z9J - BPH B854 & 0.4576 & 0.0620 & 0.0623 & -0.4127 & -0.1548 & -0.0249 & 0.0998 & -0.0261 & 0.1124 & 0.0038 & -0.0918 & -0.0522 & 0.0373 & 0.0087 & -0.0034 & 0.0028 \\
\hline 1Z9K - BPH B854 & 0.6892 & 0.0605 & 0.0158 & -0.5247 & -0.1450 & -0.0481 & 0.4192 & -0.0186 & 0.2603 & 0.0048 & -0.2525 & 0.0398 & 0.0415 & 0.0243 & 0.0003 & 0.0092 \\
\hline 2BNP - BH1 B1304 & 0.7644 & 0.0621 & 0.3442 & -0.6447 & -0.1053 & -0.0007 & 0.1599 & -0.1166 & 0.4476 & 0.0481 & 0.1255 & 0.3483 & 0.1023 & -0.0372 & 0.1006 & 0.2032 \\
\hline 2BNS - BH1 B1304 & 0.7404 & 0.0707 & 0.2899 & -0.6232 & -0.1415 & -0.0034 & 0.2079 & -0.1117 & 0.4674 & 0.0346 & 0.0597 & 0.4054 & 0.1011 & -0.0770 & 0.0538 & 0.1775 \\
\hline 2BOZ - BH1 M1311 & 0.6823 & 0.0607 & 0.2843 & -0.3719 & -0.0186 & -0.1212 & 0.4737 & -0.0836 & 0.4234 & 0.0233 & 0.1558 & 0.2618 & 0.2660 & -0.0777 & -0.0403 & 0.0895 \\
\hline 2GMR - BPH M310 & 0.5600 & 0.0464 & 0.2166 & -0.2801 & -0.0664 & -0.0647 & 0.4180 & -0.0709 & 0.5242 & 0.0365 & 0.1107 & 0.4456 & 0.1985 & -0.0726 & 0.0232 & 0.1372 \\
\hline 2GNU - BPH M1304 & 0.8351 & 0.0656 & 0.4146 & -0.6099 & -0.0330 & -0.0531 & 0.3663 & -0.1245 & 0.5375 & 0.0372 & 0.1976 & 0.3524 & 0.2408 & -0.1601 & -0.0172 & 0.2045 \\
\hline 2HG3 - BPH M401 & 0.6027 & 0.0515 & 0.2929 & -0.3664 & 0.0443 & -0.1195 & 0.3383 & -0.1122 & 0.4938 & 0.0313 & 0.1189 & 0.3870 & 0.2486 & -0.0764 & 0.0098 & 0.1107 \\
\hline 2HG9 - BPH M401 & 0.6426 & 0.0562 & 0.3175 & -0.3509 & -0.0382 & -0.1122 & 0.4107 & -0.0796 & 0.5306 & 0.0236 & 0.0699 & 0.4711 & 0.1613 & -0.1209 & -0.0430 & 0.1110 \\
\hline $2 \mathrm{HH} 1$ - BPH M401 & 0.5507 & 0.0606 & 0.1233 & -0.3560 & 0.0226 & -0.1568 & 0.3533 & -0.1069 & 0.5292 & 0.0209 & -0.0164 & 0.4799 & 0.1518 & -0.1277 & 0.0459 & 0.0900 \\
\hline $2 \mathrm{HHK}-\mathrm{BPH}$ M401 & 0.5356 & 0.0599 & 0.1565 & -0.3571 & -0.0382 & -0.1389 & 0.3258 & -0.0889 & 0.5178 & 0.0253 & 0.1094 & 0.3989 & 0.2169 & -0.1700 & -0.0871 & 0.1162 \\
\hline 2HIT - BPH M401 & 0.6694 & 0.0621 & 0.1728 & -0.4422 & 0.0624 & -0.1696 & 0.4260 & -0.0928 & 0.5368 & 0.0297 & 0.2362 & 0.3678 & 0.2358 & -0.1733 & 0.0310 & 0.1023 \\
\hline $2 \mathrm{HJ} 6-\mathrm{BPH}$ M401 & 0.6634 & 0.0563 & 0.1344 & -0.4063 & -0.0837 & -0.1381 & 0.4664 & -0.1153 & 0.5028 & 0.0228 & 0.0937 & 0.4203 & 0.1697 & -0.1745 & 0.0281 & 0.0857 \\
\hline 2J8C - BPH M1308 & 0.6741 & 0.0483 & 0.3748 & -0.3684 & -0.1265 & -0.1359 & 0.3649 & -0.1027 & 0.5884 & 0.0494 & 0.2864 & 0.4266 & 0.1981 & -0.1021 & -0.1219 & 0.1328 \\
\hline 2J8D - BPH M1317 & 0.6850 & 0.0578 & 0.2968 & -0.4257 & -0.0793 & -0.1188 & 0.4088 & -0.1112 & 0.5887 & 0.0749 & 0.1765 & 0.3192 & 0.3995 & -0.1697 & -0.0568 & 0.1478 \\
\hline 2JJO - BPH M1307 & 0.7963 & 0.0523 & 0.2597 & -0.5674 & -0.0506 & -0.1426 & 0.4551 & -0.1210 & 0.4368 & 0.0171 & 0.0295 & 0.3882 & 0.1404 & -0.0617 & 0.1082 & 0.0631 \\
\hline 2RCR - BPH M500 & 0.3875 & 0.0566 & 0.2104 & -0.1877 & -0.1366 & -0.1169 & 0.1746 & -0.0887 & 0.5525 & 0.0345 & 0.0376 & 0.4619 & 0.1950 & -0.1742 & -0.1345 & -0.0637 \\
\hline 2UWS - BPH M1312 & 0.8828 & 0.0948 & 0.4818 & -0.6116 & -0.0038 & -0.1703 & 0.3730 & -0.0701 & 0.8523 & 0.1341 & 0.1989 & 0.3648 & 0.7035 & 0.1201 & -0.2097 & -0.0203 \\
\hline 2UWT - BPH M1312 & 0.7803 & 0.0705 & 0.3520 & -0.5302 & -0.0398 & -0.1740 & 0.4092 & -0.0677 & 0.7932 & 0.0802 & 0.2844 & 0.5639 & 0.4109 & -0.2402 & -0.0605 & -0.0055 \\
\hline 2UWU - BPH M1312 & 0.6877 & 0.0691 & 0.3003 & -0.3696 & -0.0450 & -0.1059 & 0.4745 & -0.0879 & 0.4985 & 0.0823 & 0.2193 & 0.1710 & 0.3037 & -0.2473 & -0.0484 & 0.1245 \\
\hline 2UWV - BPH M1313 & 0.7058 & 0.0700 & 0.3384 & -0.3965 & -0.0786 & -0.0206 & 0.4649 & -0.0607 & 0.6215 & 0.0890 & 0.3518 & 0.3553 & 0.3323 & -0.0530 & -0.1511 & 0.0107 \\
\hline $2 U W W$ - BPH M1311 & 0.6725 & 0.0642 & 0.3353 & -0.3396 & -0.0520 & -0.1169 & 0.4516 & -0.0653 & 0.5931 & 0.0709 & 0.3621 & 0.3376 & 0.2623 & -0.1603 & -0.0977 & 0.0509 \\
\hline $2 U X 3-B P H ~ M 1313$ & 0.7602 & 0.0732 & 0.4233 & -0.4036 & -0.0809 & -0.1413 & 0.4473 & -0.0956 & 0.7002 & 0.0882 & 0.3255 & 0.4178 & 0.4157 & -0.1169 & 0.0249 & 0.1506 \\
\hline $2 \mathrm{UX4}-\mathrm{BPH}$ M1311 & 0.6750 & 0.0694 & 0.3271 & -0.4305 & -0.0707 & -0.1210 & 0.3687 & -0.0879 & 0.8679 & 0.0936 & 0.4667 & 0.6598 & 0.2380 & -0.1131 & -0.1621 & -0.0662 \\
\hline $2 \mathrm{UX5}-\mathrm{BPH} \mathrm{M} 1314$ & 0.7420 & 0.0606 & 0.4360 & -0.4039 & -0.0411 & -0.1958 & 0.3889 & -0.0781 & 0.6472 & 0.1107 & 0.1656 & 0.3610 & 0.2945 & -0.3535 & 0.1368 & 0.1753 \\
\hline 2 UXJ - BPH M1313 & 0.7224 & 0.0672 & 0.2062 & -0.5610 & -0.0833 & -0.1338 & 0.3709 & -0.0460 & 0.7572 & 0.0802 & 0.3951 & 0.5798 & 0.1771 & -0.1182 & -0.1876 & 0.0227 \\
\hline
\end{tabular}




\begin{tabular}{|c|c|c|c|c|c|c|c|c|c|c|c|c|c|c|c|c|}
\hline $2 \mathrm{UXK}$ - BPH M1311 & 0.6889 & 0.0748 & 0.2960 & -0.4656 & -0.0381 & -0.1645 & 0.3651 & -0.0921 & 0.5881 & 0.0972 & 0.1590 & 0.4292 & 0.1877 & -0.2840 & 0.0681 & 0.1258 \\
\hline 2UXL - BPH M1309 & 0.7144 & 0.0705 & 0.2812 & -0.5855 & 0.0573 & -0.1289 & 0.2307 & -0.1237 & 0.8223 & 0.1224 & 0.4098 & 0.3948 & 0.3840 & -0.3106 & 0.2822 & 0.1698 \\
\hline 2UXM - BPH M1314 & 0.6799 & 0.0523 & 0.3198 & -0.4548 & -0.1638 & -0.0883 & 0.3172 & -0.1338 & 0.7677 & 0.0970 & 0.1840 & 0.4324 & 0.4370 & 0.0210 & 0.1088 & 0.4065 \\
\hline 2WX5 - BPH M1314 & 0.6462 & 0.0644 & 0.2893 & -0.4324 & -0.1077 & -0.1166 & 0.3388 & -0.0830 & 0.5292 & 0.0504 & 0.2450 & 0.3265 & 0.1543 & -0.1324 & 0.1600 & 0.2156 \\
\hline 3DSY - BPH M503 & 0.5895 & 0.0546 & 0.3120 & -0.3672 & -0.0493 & -0.0484 & 0.3319 & -0.0214 & 0.6393 & 0.0245 & 0.1634 & 0.5001 & 0.3282 & -0.1283 & 0.0312 & 0.0822 \\
\hline 3DTA - BPH M503 & 0.5901 & 0.0547 & 0.3118 & -0.3678 & -0.0498 & -0.0494 & 0.3320 & -0.0222 & 0.6390 & 0.0246 & 0.1638 & 0.4999 & 0.3276 & -0.1282 & 0.0312 & 0.0821 \\
\hline 3DTR - BPH M503 & 0.5896 & 0.0547 & 0.3114 & -0.3675 & -0.0497 & -0.0497 & 0.3321 & -0.0215 & 0.6393 & 0.0246 & 0.1635 & 0.5002 & 0.3282 & -0.1282 & 0.0318 & 0.0817 \\
\hline 3DTS - BPH M504 & 0.5895 & 0.0546 & 0.3121 & -0.3672 & -0.0505 & -0.0492 & 0.3313 & -0.0219 & 0.6403 & 0.0247 & 0.1631 & 0.5010 & 0.3283 & -0.1295 & 0.0329 & 0.0821 \\
\hline 3DU2 - BPH M503 & 0.5896 & 0.0548 & 0.3119 & -0.3673 & -0.0493 & -0.0493 & 0.3319 & -0.0214 & 0.6393 & 0.0246 & 0.1636 & 0.5000 & 0.3281 & -0.1287 & 0.0312 & 0.0818 \\
\hline 3DU3 - BPH M503 & 0.5894 & 0.0546 & 0.3113 & -0.3675 & -0.0499 & -0.0498 & 0.3318 & -0.0213 & 0.6398 & 0.0247 & 0.1626 & 0.5008 & 0.3282 & -0.1288 & 0.0316 & 0.0828 \\
\hline 3DUQ - BPH M503 & 0.5897 & 0.0546 & 0.3111 & -0.3678 & -0.0507 & -0.0497 & 0.3320 & -0.0216 & 0.6393 & 0.0245 & 0.1631 & 0.5007 & 0.3269 & -0.1294 & 0.0325 & 0.0824 \\
\hline 4RCR - BPH M312 & 0.3044 & 0.0326 & -0.0329 & -0.0824 & 0.0171 & 0.0362 & 0.2875 & -0.0222 & 0.7746 & 0.0616 & -0.0862 & 0.0243 & 0.5681 & 0.2713 & 0.3825 & 0.2221 \\
\hline
\end{tabular}

\subsection{Blastochloris viridis bacteriopheophytin cofactor NSDs}

Table 65: Minimum basis normal-mode displacements and conformational parameters of all ФA cofactors from crystal structures of Blastochloris viridis.

\begin{tabular}{|c|c|c|c|c|c|c|c|c|c|c|c|c|c|c|c|c|}
\hline Cofactor ID & Dip & $\delta$ iip & B2g & B1g & $\mathrm{Eu}(\mathrm{x})$ & $\mathrm{Eu}(\mathrm{y})$ & A1g & $A 2 g$ & Doop & Soop & $\mathrm{B} 2 \mathrm{u}$ & B1u & $\mathrm{A} 2 \mathrm{u}$ & $\mathrm{Eg}(\mathrm{x})$ & $\mathrm{Eg}(\mathrm{y})$ & A1u \\
\hline 1DXR - BPB L402 & 0.5550 & 0.0484 & 0.4054 & -0.1767 & -0.0925 & -0.0193 & 0.3123 & -0.0775 & 0.4422 & 0.0324 & -0.1704 & 0.3278 & 0.0499 & -0.2040 & -0.0604 & 0.1063 \\
\hline 1PRC - BPB L606 & 0.5477 & 0.0398 & 0.3293 & -0.3321 & -0.0897 & -0.0962 & 0.2221 & -0.1211 & 0.6134 & 0.0363 & -0.4735 & 0.2565 & 0.0699 & -0.2708 & 0.0634 & 0.0628 \\
\hline 1R2C - BPB L402 & 0.5673 & 0.0451 & 0.3127 & -0.3702 & -0.1043 & -0.0157 & 0.2663 & -0.0709 & 0.4630 & 0.0311 & -0.0851 & 0.3559 & 0.2099 & -0.0509 & -0.1738 & 0.0602 \\
\hline 1VRN - BPB L406 & 0.5088 & 0.0427 & 0.3084 & -0.2362 & -0.1420 & -0.0686 & 0.2820 & -0.0607 & 0.4535 & 0.0276 & -0.1886 & 0.3909 & 0.0413 & -0.0890 & -0.0591 & 0.0646 \\
\hline $215 N-B P B M 402$ & 0.7830 & 0.0708 & 0.4503 & -0.5009 & -0.1308 & -0.0857 & 0.3359 & -0.1489 & 0.5253 & 0.0374 & -0.1233 & 0.4507 & 0.0667 & -0.1348 & 0.0415 & 0.1822 \\
\hline 2JBL - BPB L1276 & 0.4210 & 0.0466 & 0.2381 & -0.1313 & -0.1354 & -0.0328 & 0.2815 & -0.0679 & 0.5000 & 0.0241 & -0.2878 & 0.3875 & -0.0277 & -0.1074 & -0.0111 & 0.0678 \\
\hline 2PRC - BPB L402 & 0.5959 & 0.0504 & 0.4636 & -0.0654 & -0.1369 & -0.0128 & 0.3349 & -0.0698 & 0.6355 & 0.0219 & -0.3995 & 0.4354 & 0.0416 & -0.2018 & 0.0091 & 0.1102 \\
\hline 2WJM - BPB L1276 & 0.5713 & 0.0535 & 0.3012 & -0.2175 & -0.0120 & -0.0411 & 0.4292 & -0.0479 & 0.5252 & 0.0408 & -0.0998 & 0.4297 & 0.1240 & -0.1812 & 0.0332 & 0.1786 \\
\hline 2WJN - BPB L1276 & 0.6247 & 0.0578 & 0.4269 & -0.2220 & -0.0914 & -0.0224 & 0.3799 & -0.0745 & 0.4559 & 0.0372 & -0.1495 & 0.3497 & 0.0463 & -0.1659 & -0.0656 & 0.1709 \\
\hline 3D38 - BPB L402 & 0.7689 & 0.0805 & 0.3978 & -0.4974 & -0.1474 & -0.0600 & 0.3669 & -0.1599 & 0.6324 & 0.0513 & -0.2593 & 0.3295 & -0.2208 & -0.1107 & -0.3965 & 0.0774 \\
\hline 3G7F - BPB L402 & 0.6681 & 0.0801 & 0.3681 & -0.3787 & -0.1850 & -0.0365 & 0.3274 & -0.1573 & 0.4786 & 0.0414 & -0.2322 & 0.3386 & -0.0838 & -0.1502 & -0.1619 & 0.0680 \\
\hline 3PRC - BPB L402 & 0.5293 & 0.0478 & 0.4453 & -0.0269 & -0.1148 & -0.0157 & 0.2440 & -0.0903 & 0.5328 & 0.0229 & -0.2932 & 0.3869 & 0.0023 & -0.1876 & -0.0474 & 0.1037 \\
\hline 5PRC - BPB L402 & 0.5065 & 0.0465 & 0.2843 & -0.0699 & -0.1593 & 0.0310 & 0.3712 & -0.0820 & 0.5783 & 0.0204 & -0.2605 & 0.4453 & 0.0072 & -0.2433 & -0.0218 & 0.0925 \\
\hline $6 P R C-B P B L 402$ & 0.6010 & 0.0419 & 0.3809 & -0.2359 & -0.1272 & -0.0174 & 0.3702 & -0.0832 & 0.5212 & 0.0275 & -0.2677 & 0.3580 & 0.0092 & -0.2462 & -0.0404 & 0.0971 \\
\hline 7PRC - BPB L402 & 0.3762 & 0.0500 & 0.1562 & -0.1645 & -0.1356 & -0.0428 & 0.2467 & -0.0948 & 0.6058 & 0.0321 & -0.3472 & 0.3809 & 0.1019 & -0.2804 & 0.0159 & 0.1099 \\
\hline
\end{tabular}

Table 66: Minimum basis normal-mode displacements and conformational parameters of all ФB cofactors from crystal structures of Blastochloris viridis.

\begin{tabular}{|c|c|c|c|c|c|c|c|c|c|c|c|c|c|c|c|c|}
\hline Cofactor ID & Dip & Sip & B2g & B1g & $\mathrm{Eu}(\mathrm{x})$ & Eu(y) & A1g & A2g & Doop & Soop & $\mathrm{B} 2 \mathrm{u}$ & B1u & $\mathrm{A} 2 \mathrm{u}$ & $\mathrm{Eg}(\mathrm{x})$ & $\mathrm{Eg}(\mathrm{y})$ & A1u \\
\hline 1DXR - BPB M402 & 0.5230 & 0.0433 & 0.1625 & -0.3755 & -0.0506 & -0.0309 & 0.3116 & -0.0740 & 0.5691 & 0.0282 & -0.0636 & 0.5018 & 0.0706 & -0.1905 & 0.0906 & 0.1358 \\
\hline 1PRC - BPB M605 & 0.5920 & 0.0591 & 0.1832 & -0.4341 & -0.1320 & 0.0075 & 0.3225 & -0.0840 & 0.4007 & 0.0457 & -0.0268 & 0.3331 & -0.0328 & -0.0832 & -0.0965 & 0.1777 \\
\hline 1R2C - BPB M401 & 0.5710 & 0.0434 & 0.3132 & -0.3227 & -0.1425 & -0.0108 & 0.3118 & -0.0780 & 0.4208 & 0.0214 & 0.0370 & 0.3787 & 0.1340 & -0.0425 & -0.0748 & 0.0833 \\
\hline 1VRN - BPB M405 & 0.5236 & 0.0419 & 0.3217 & -0.2688 & -0.1032 & -0.0602 & 0.2810 & -0.0718 & 0.5748 & 0.0292 & -0.1484 & 0.5057 & -0.0066 & -0.1572 & 0.0928 & 0.1390 \\
\hline $215 N-B P B L 402$ & 0.6445 & 0.0764 & 0.3891 & -0.3713 & -0.1318 & -0.0690 & 0.2874 & -0.1463 & 0.5267 & 0.0442 & -0.2353 & 0.3229 & -0.0958 & -0.2113 & -0.2395 & 0.0815 \\
\hline 2JBL - BPB M1325 & 0.5059 & 0.0412 & 0.2175 & -0.3309 & -0.1331 & -0.0240 & 0.2730 & -0.0795 & 0.5901 & 0.0266 & -0.2301 & 0.5097 & -0.0416 & -0.1432 & 0.0374 & 0.1091 \\
\hline
\end{tabular}




\begin{tabular}{|c|c|c|c|c|c|c|c|c|c|c|c|c|c|c|c|c|}
\hline 2PRC - BPB M401 & 0.5870 & 0.0467 & 0.1639 & -0.4421 & -0.0553 & -0.0082 & 0.3389 & -0.0651 & 0.6715 & 0.0451 & -0.2385 & 0.5705 & 0.1238 & -0.1405 & 0.0218 & 0.1815 \\
\hline 2WJM - BPB M1326 & 0.5635 & 0.0553 & 0.2498 & -0.3249 & 0.0175 & -0.0130 & 0.3813 & -0.0607 & 0.6802 & 0.0519 & -0.0695 & 0.5782 & 0.1291 & -0.0954 & 0.1537 & 0.2722 \\
\hline 2 WJN - BPB M1326 & 0.5586 & 0.0498 & 0.1543 & -0.3530 & -0.0307 & -0.0187 & 0.3967 & -0.0704 & 0.6782 & 0.0477 & -0.0157 & 0.5824 & 0.0822 & -0.1556 & 0.0741 & 0.2898 \\
\hline 3D38 - BPB M402 & 0.8113 & 0.0821 & 0.3403 & -0.6448 & -0.1734 & -0.0398 & 0.2638 & -0.1595 & 0.8335 & 0.0590 & 0.1359 & 0.6534 & 0.2675 & -0.2715 & 0.2305 & 0.2257 \\
\hline 3G7F - BPB M402 & 0.8951 & 0.0654 & 0.3056 & -0.7375 & -0.1970 & 0.0172 & 0.3137 & -0.1625 & 0.4615 & 0.0560 & -0.1640 & 0.2980 & 0.1402 & -0.1804 & 0.0204 & 0.2113 \\
\hline 3PRC - BPB M401 & 0.5865 & 0.0421 & 0.1601 & -0.4654 & -0.0634 & -0.0652 & 0.2975 & -0.0708 & 0.6674 & 0.0332 & -0.0180 & 0.6014 & 0.1107 & -0.2076 & 0.0206 & 0.1663 \\
\hline 5PRC - BPB M401 & 0.5389 & 0.0478 & 0.0619 & -0.4355 & -0.0837 & -0.0380 & 0.2888 & -0.0710 & 0.6495 & 0.0405 & -0.3557 & 0.4869 & -0.0217 & -0.1976 & -0.0672 & 0.1191 \\
\hline 6PRC - BPB M401 & 0.5384 & 0.0433 & 0.1503 & -0.3857 & -0.1078 & -0.0321 & 0.3169 & -0.0734 & 0.7074 & 0.0393 & -0.2705 & 0.6055 & 0.0200 & -0.1761 & -0.0783 & 0.1519 \\
\hline 7PRC - BPB L401 & 0.6320 & 0.0404 & 0.1898 & -0.5306 & -0.1128 & 0.0184 & 0.2443 & -0.0959 & 0.7928 & 0.0378 & -0.1440 & 0.7150 & 0.0217 & -0.2679 & -0.1161 & 0.1036 \\
\hline
\end{tabular}

